Supporting Information for:

\title{
Asymmetric Hydrogenation of $\beta$-Aryloxy/Alkoxy Cinnamic Nitriles and Esters
}

\author{
Duanyang Kong, Meina Li, Rui Wang, Guofu Zi, and Guohua Hou* \\ College of Chemistry, Beijing Normal University, Beijing 100875, China
}

Table of contents

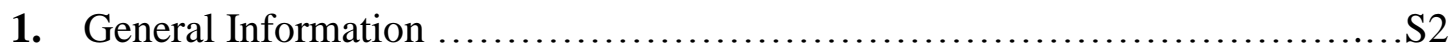

2. General procedure for the preparation of compounds $\mathbf{1}$ and $3 \ldots \ldots \ldots \ldots \ldots . . .52$

3. General procedure for asymmetric hydrogenation of compounds $\mathbf{1}$ and $3 \ldots \ldots \ldots$....S5

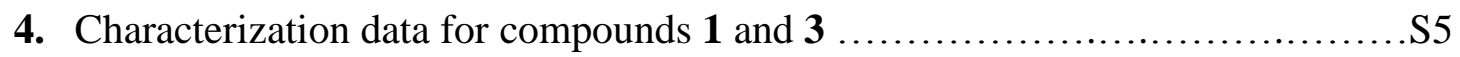

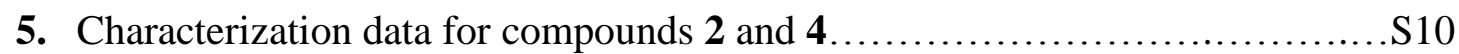

6. Procedure for the synthesis of $(S)$-Nisoxetine............................ 18

7. NMR, GC and HPLC spectra of all compounds............................ 21 


\section{General Information}

All the air or moisture sensitive reactions and manipulations were performed by using standard Schlenk techniques and in a nitrogen-filled glovebox. DME, THF, dioxane and toluene were distilled from sodium benzophenone ketyl. $\mathrm{CH}_{2} \mathrm{Cl}_{2}$ was distilled from calcium hydride. Anhydrous $\mathrm{MeOH}$ was distilled from magnesium. ${ }^{1} \mathrm{H}$ NMR and ${ }^{13} \mathrm{C}$ NMR spectra were recorded on Bruker AV (400 MHz) spectrometers. $\mathrm{CDC}_{3}$ was the solvent used for the NMR analysis, with TMS as the internal standard. Chemical shifts were reported upfield to TMS (0.00 ppm) for ${ }^{1} \mathrm{H}$ NMR. Data is represented as follows: chemical shift, integration, multiplicity $(\mathrm{s}=$ singlet, $\mathrm{d}=$ doublet, $\mathrm{dd}=$ double of doublets, $\mathrm{t}=$ triplet, $\mathrm{q}=$ quartet, $\mathrm{m}=$ multiplet $)$ and coupling constants $(J)$ in Hertz $(\mathrm{Hz})$. Optical rotation was determined using Autopol III Automatic polarimeter (Rudolph research Analyical). GC analysis was conducted on an Agilent 7890A series instrument. HPLC analysis was conducted on Agilent 1260 series instrument. HRMS were recorded on a Waters LCT Premier XE mass spectrometer with APCI or ESI.

\section{General procedure for the preparation of compounds 1 and 3}

Method A: 1a-1j were prepared by the following protocol. ${ }^{[1]}$

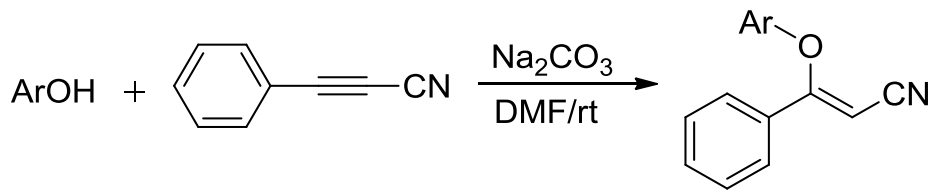

(Z)-1

To a $25 \mathrm{~mL}$ Schlenk tube was added $\mathrm{Na}_{2} \mathrm{CO}_{3}(2.0 \mathrm{mmol})$, 3-phenylpropiolonitrile (2.0 mmol), substituted phenol (2.4 mmol) and DMF (4 mL) under an air atmosphere. After the reaction was completed at room temperature, it was diluted with ethyl acetate. The resulting solution was directly filtered through a pad of Celite and concentrated under reduced pressure. The residue was purified by flash silica gel chromatography.

Method B: 1k-1m were prepared by the following protocol. ${ }^{[1]}$ 


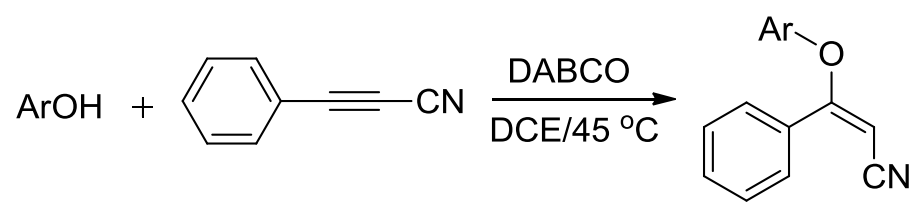

$(E)-1$

To a $25 \mathrm{~mL}$ Schlenk tube was added DABCO (2.0 mmol), 3-phenylpropiolonitrile (2.0 mmol), substituted phenol $(2.4 \mathrm{mmol})$ and DCE $(4 \mathrm{~mL})$ under an air atmosphere. After the reaction was completed at $45{ }^{\circ} \mathrm{C}$, it was cooled to room temperature and diluted with ethyl acetate. The resulting solution was directly filtered through a pad of Celite and concentrated under reduced pressure. The residue was purified by flash silica gel chromatography.

Method C: 1n-1p were prepared by following the procedure.

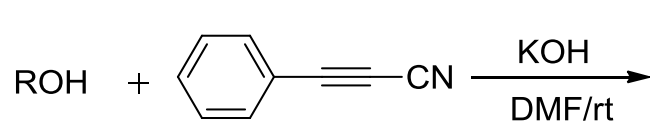<smiles>[R]O/C(=C\C#N)c1ccccc1</smiles>

A mixture of alcohol (2 mmol), $\mathrm{KOH}(0.2 \mathrm{mmol})$ and 3-phenylpropiolonitrile (2 mmol) was stirred in DMF (4 $\mathrm{mL}$ ) at $\mathrm{rt}$. After the reaction was completed, the reaction mixture was diluted with $\mathrm{H}_{2} \mathrm{O}$ and extracted with ethyl acetate. The organic extracts were washed with $\mathrm{H}_{2} \mathrm{O}$ and dried $\left(\mathrm{MgSO}_{4}\right)$. The solvent was evaporated, the residue was purified by column chromatography.

Method D: 1q was prepared by following the procedure. ${ }^{[2]}$<smiles>N#C/C=C(/c1ccccc1)n1cccc1C(O)O[Mg]</smiles>

A mixture of pyrrole $(2 \mathrm{mmol}), \mathrm{KOH}(0.1 \mathrm{mmol})$ and 3-phenylpropiolonitrile (2 mmol) was stirred in DMSO (4 $\mathrm{mL})$ at $\mathrm{rt}$ for $3 \mathrm{~h}$. The reaction mixture was diluted with $\mathrm{H}_{2} \mathrm{O}$ and extracted with ethyl acetate. The organic extracts were washed with $\mathrm{H}_{2} \mathrm{O}$ and dried $\left(\mathrm{MgSO}_{4}\right)$. The solvent was evaporated, the residue was purified by column chromatography.

Method E: 1r was synthesized by following the procedure. ${ }^{[3,4]}$ 


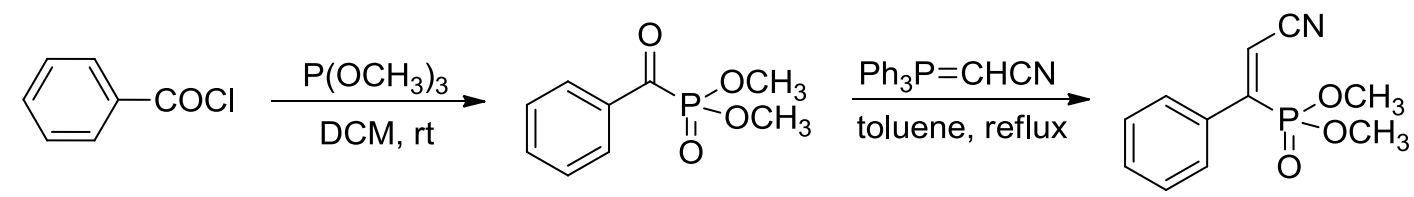

To an oven-dried $100 \mathrm{ml}$ round bottom flask equipped with a magnetic stir bar under $\mathrm{N}_{2}$, the benzoyl chloride $(5 \mathrm{mmol})$ was dissolved in $\mathrm{CH}_{2} \mathrm{Cl}_{2}(30 \mathrm{ml})$. Then trimethylphosphite $(5.5 \mathrm{mmol})$ was added dropwise. The resulting solution was stirred for $24 \mathrm{~h}$, and the reaction was concentrated under reduced pressure. The oil obtained was purified by vacuum distillation.

The above $\alpha$-keto phosphonate $(2 \quad \mathrm{mmol})$ and 2-(triphenylphosphoranylidene)-acetonitrile (3 $\mathrm{mmol})$ were dissolved in toluene (15 $\mathrm{ml}$ ) in a round bottle flask equipped with a reflux condenser. The mixture was heated to reflux for $12 \mathrm{~h}$. Then the solvent was removed in vacuo. The residue was purified by silica gel column chromatography.

Method F: 3a-3f were prepared by the following protocol. ${ }^{[5]}$

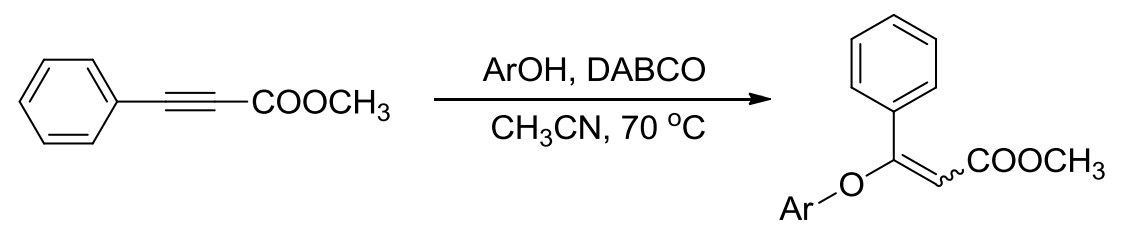

To a $50 \mathrm{~mL}$ Schlenk tube was added DABCO (2.0 mmol), substituted phenol (2.4 mmol) and acetonitrile $(10 \mathrm{~mL})$ under $\mathrm{N}_{2}$. The resulting solution heated to $70{ }^{\circ} \mathrm{C}$, followed by addition of methyl 3-phenylpropiolate $(2.0 \mathrm{mmol})$. The resulting solution was stirred at $70{ }^{\circ} \mathrm{C}$ for $20-24 \mathrm{~h}$, and the solvent was removed in vacuo. The residue was purified by silica gel chromatography to afford products $\mathbf{3 a - 3 f}$.

Method G: 3g was synthesized by following the procedure. ${ }^{[6]}$

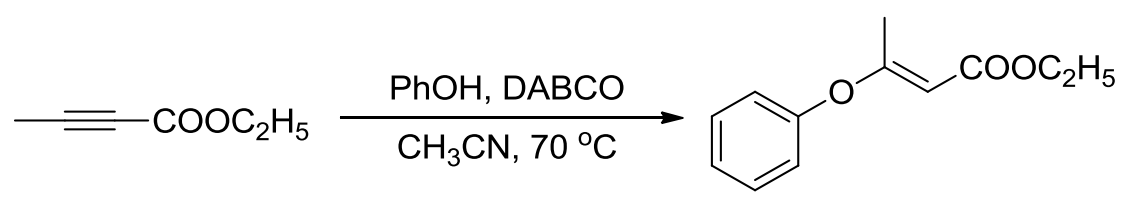

To a $50 \mathrm{~mL}$ Schlenk tube was added DABCO $(2.0 \mathrm{mmol})$, phenol $(2.4 \mathrm{mmol})$ and acetonitrile $(10 \mathrm{~mL})$ under $\mathrm{N}_{2}$. The resulting solution heated to $70{ }^{\circ} \mathrm{C}$, followed by addition of ethyl but-2-ynoate $(2.0 \mathrm{mmol})$. The resulting solution was stirred at $70{ }^{\circ} \mathrm{C}$ for $2 \mathrm{~h}$, and the solvent was removed in vacuo. The residue was purified by silica gel 
chromatography to afford products $\mathbf{3 g}$.

\section{General procedure for asymmetric hydrogenation of compounds 1 and 3.}

A stock solution was made by mixing $[\mathrm{Rh}(\mathrm{COD}) \mathrm{Cl}]_{2}$ with $(R, R)$-f- spiroPhos in a 1:1.1 molar ratio of metal $\mathrm{Rh} /(R, R)-\mathrm{f}-$ spiroPhos in $\mathrm{CH}_{2} \mathrm{Cl}_{2}$ at room temperature for 20 min in a nitrogen-filled glovebox. An aliquot of the catalyst solution $(1.0 \mathrm{~mL}, 0.001$ mmol) was transferred by syringe into the vials charged with different substrates $(0.1$ mmol for each) in anhydrous $\mathrm{CH}_{2} \mathrm{Cl}_{2}(2.0 \mathrm{ml})$. The vials were then placed into a steel autoclave. The inert atmosphere was replaced by $\mathrm{H}_{2}$ and the reaction mixture was stirred. The hydrogen gas was released slowly and carefully. The solution was concentrated and passed through a short column of silica gel to remove the metal complex. The ee values of all products were determined by HPLC or GC analysis on a chiral stationary phase.

\section{Characterization data for compounds 1and 3.}

(Z)-3-phenoxy-3-phenylacrylonitrile (1a): White solid; MP: $86-88{ }^{\circ} \mathrm{C}$; Yield: $99 \%$;

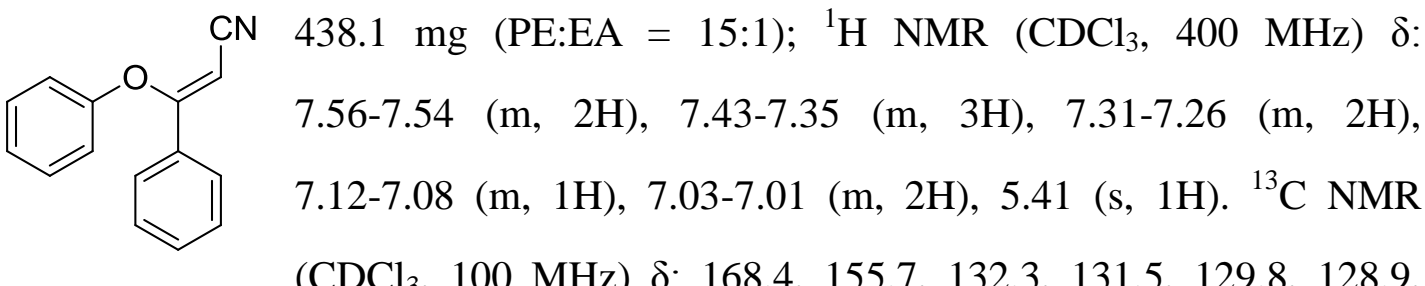
$126.9,124.3,118.2,115.1,81.8 .^{[1]}$

(Z)-3-phenyl-3-(o-tolyloxy)acrylonitrile (1b): colorless oil; Yield: $95 \%$; $447.0 \mathrm{mg}$<smiles>Cc1ccccc1O/C(=C\C#N)c1ccccc1</smiles>
(PE:EA = 20:1); ${ }^{1} \mathrm{H} \mathrm{NMR}\left(\mathrm{CDCl}_{3}, 400 \mathrm{MHz}\right) \delta: 7.60-7.59(\mathrm{~m}, 2 \mathrm{H})$ 7.48-7.38 (m, 3H), 7.25-7.24 (m, 1H), 7.09-7.08 (m, 2H), 6.84-6.82 (m, 2H), $5.31(\mathrm{~s}, 1 \mathrm{H}), 2.40(\mathrm{~s}, 3 \mathrm{H}) .{ }^{13} \mathrm{C} \mathrm{NMR}\left(\mathrm{CDCl}_{3}\right.$, $100 \mathrm{MHz}) \delta: 168.9,154.2,133.2,132.0,131.9,129.5,129.4$,

$127.6,127.1,125.6,118.9,115.3,79.0,16.7 .^{[1]}$

(Z)-3-(2-methoxyphenoxy)-3-phenylacrylonitrile (1c): White solid; MP: $93-95{ }^{\circ} \mathrm{C}$

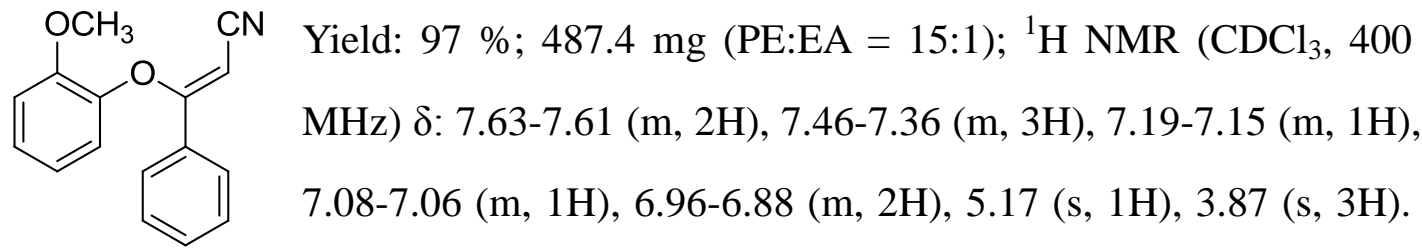


${ }^{13} \mathrm{C}$ NMR $\left(\mathrm{CDCl}_{3}, 100 \mathrm{MHz}\right) \delta: 169.7,151.4,144.4,133.4,131.8,129.5,129.2,127.4$, 126.9, $121.5,121.4,115.5,113.2,56.5$. TOF-HRMS Calcd. for $\mathrm{C}_{16} \mathrm{H}_{14} \mathrm{NO}_{2}\left[\mathrm{M}+\mathrm{H}^{+}\right]$: 252.1019, found 252.1017.

(Z)-3-(2-chlorophenoxy)-3-phenylacrylonitrile (1d): colorless oil; Yield: $96 \%$;<smiles>N#C/C=C(/Oc1ccccc1Cl)c1ccccc1</smiles>
$490.9 \mathrm{mg}(\mathrm{PE}: \mathrm{EA}=15: 1) ;{ }^{1} \mathrm{H} \mathrm{NMR}\left(\mathrm{CDCl}_{3}, 400 \mathrm{MHz}\right) \delta: 7.63-7.61$ $(\mathrm{m}, 2 \mathrm{H}), 7.45-7.38(\mathrm{~m}, 3 \mathrm{H}), 7.21-7.11(\mathrm{~m}, 3 \mathrm{H}), 7.03-7.01(\mathrm{~m}, 1 \mathrm{H})$, $5.34(\mathrm{~s}, 1 \mathrm{H}) .{ }^{13} \mathrm{C} \mathrm{NMR}\left(\mathrm{CDCl}_{3}, 100 \mathrm{MHz}\right) \delta: 168.4,151.4,132.5$, $132.2,131.3,129.5,128.5,127.4,126.7,125.9,121.1,115.1,80.2$. TOF-HRMS Calcd. for $\mathrm{C}_{15} \mathrm{H}_{11} \mathrm{NOCl}\left[\mathrm{M}+\mathrm{H}^{+}\right]$: 256.0523, found 256.0523.

(Z)-3-(3-chlorophenoxy)-3-phenylacrylonitrile (1e): colorless oil; Yield: $95 \%$;<smiles>N#C/C=C(/Oc1cccc(Cl)c1)c1ccccc1</smiles>
$485.8 \mathrm{mg}$ (PE:EA $=15: 1) ;{ }^{1} \mathrm{H}$ NMR $\left(\mathrm{CDCl}_{3}, 400 \mathrm{MHz}\right) \delta$ : 7.54-7.38 (m, 5H), 7.23-7.19 (m, 1H), 7.08-7.03 (m, 2H), 6.89-6.87 (m, 1H), $5.50(\mathrm{~s}, 1 \mathrm{H}) .{ }^{13} \mathrm{C} \mathrm{NMR}\left(\mathrm{CDCl}_{3}, 100 \mathrm{MHz}\right) \delta$ : $168.2,156.9,135.8,132.4,132.1,131.1,129.7,127.4,124.9$,

118.8, 116.5, 115.4, 84.0. TOF-HRMS Calcd. for $\mathrm{C}_{15} \mathrm{H}_{11} \mathrm{NOCl}\left[\mathrm{M}+\mathrm{H}^{+}\right]$: 256.0523, found 256.0523 .

(Z)-3-phenyl-3-(p-tolyloxy)acrylonitrile (1f): colorless oil; Yield: 97 \%; $456.4 \mathrm{mg}$<smiles>Cc1ccc(O/C(=C/C#N)c2ccccc2)cc1</smiles>
(PE:EA = 20:1); ${ }^{1} \mathrm{H}$ NMR $\left(\mathrm{CDCl}_{3}, 400 \mathrm{MHz}\right) \delta: 7.56-7.54(\mathrm{~m}$, 2H), 7.46-7.35 (m, 3H), 7.09-7.07 (m, 2H), 6.92-6.90 (m, 2H), $5.35(\mathrm{~s}, 1 \mathrm{H}), 2.29(\mathrm{~s}, 3 \mathrm{H}) .{ }^{13} \mathrm{C} \mathrm{NMR}\left(\mathrm{CDCl}_{3}, 100 \mathrm{MHz}\right) \delta$ : $169.3,154.1,134.4,132.9,132.0,130.8,129.5,127.5,118.6$, $115.9,81.8,21.3 .^{[1]}$

(Z)-3-(4-chlorophenoxy)-3-phenylacrylonitrile (1g): White solid; MP: $46-48{ }^{\circ} \mathrm{C}$;

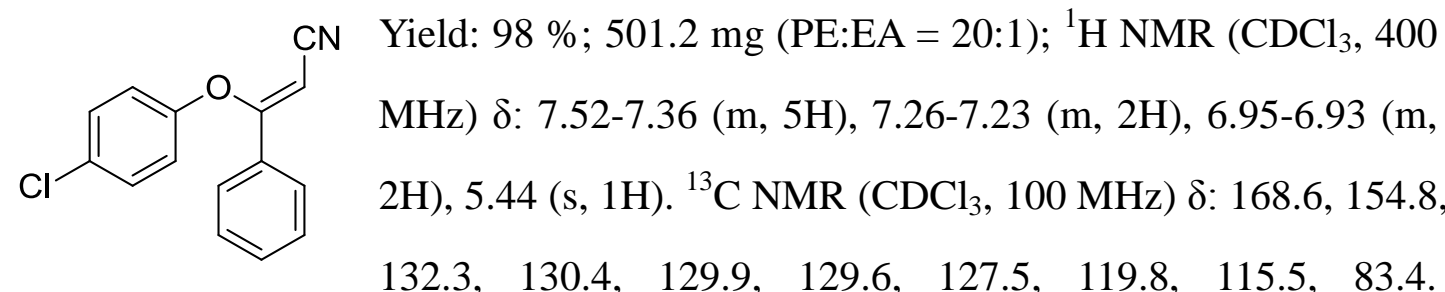
TOF-HRMS Calcd. for $\mathrm{C}_{15} \mathrm{H}_{11} \mathrm{NOCl}\left[\mathrm{M}+\mathrm{H}^{+}\right]$: 256.0523, found 256.0523.

(Z)-3-phenyl-3-(4-(trifluoromethyl)phenoxy)acrylonitrile (1h): White solid; MP: 60-62 ${ }^{\circ} \mathrm{C}$; Yield: $97 \%$; $561.1 \mathrm{mg}(\mathrm{PE}: \mathrm{EA}=15: 1)$; ${ }^{1} \mathrm{H} \mathrm{NMR}\left(\mathrm{CDCl}_{3}, 400 \mathrm{MHz}\right) \delta$ : 
7.56-7.37 (m, 7H), 7.09-7.07 (m, 2H), $5.58(\mathrm{~s}, 1 \mathrm{H}) .{ }^{13} \mathrm{C} \mathrm{NMR}\left(\mathrm{CDCl}_{3}, 100 \mathrm{MHz}\right) \delta$ :

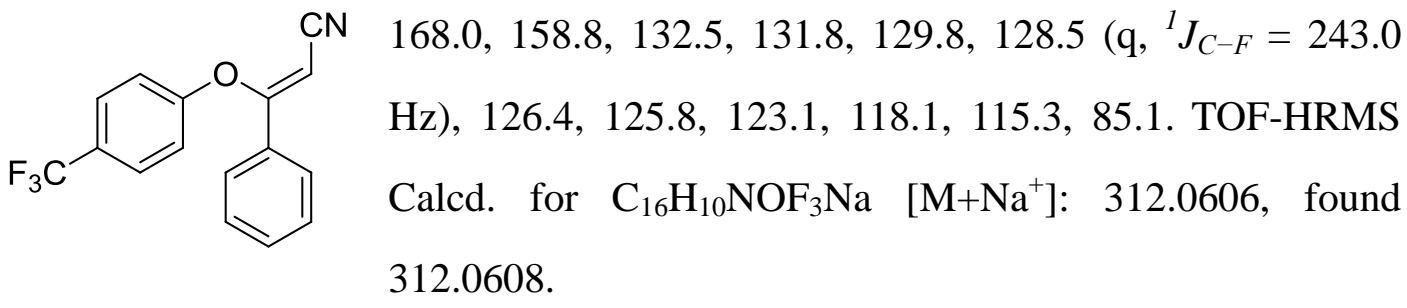

(Z)-3-(naphthalen-1-yloxy)-3-phenylacrylonitrile (1i): White solid; MP:

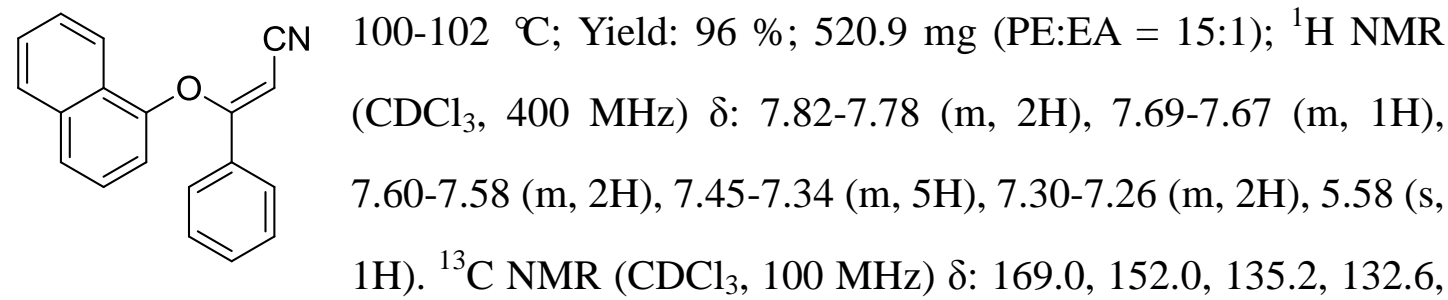
132.1, 129.6, 128.5, 127.5, 127.1, 126.3, 125.8, 124.9, 121.9, 115.6, 113.0, 82.6. TOF-HRMS Calcd. for $\mathrm{C}_{19} \mathrm{H}_{14} \mathrm{NO}\left[\mathrm{M}+\mathrm{H}^{+}\right]$: 272.1069, found 272.1070.

(Z)-3-(naphthalen-2-yloxy)-3-phenylacrylonitrile (1j): White solid; MP: $92-94{ }^{\circ} \mathrm{C}$;

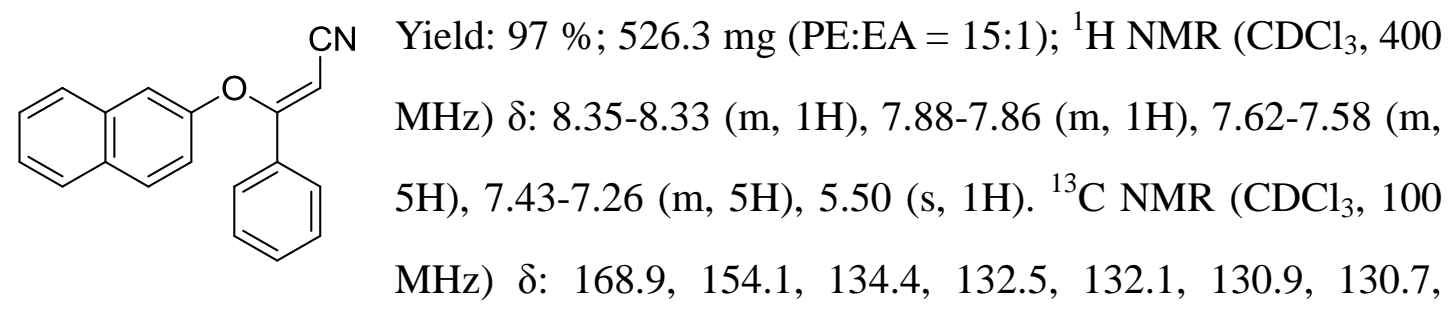
129.6, 128.4, 127.8, 127.5, 127.4, 125.7, 119.1, 115.8, 114.1, 83.7. TOF-HRMS Calcd. for $\mathrm{C}_{19} \mathrm{H}_{14} \mathrm{NO}\left[\mathrm{M}+\mathrm{H}^{+}\right]: 272.1069$, found 272.1070.

(E)-3-phenoxy-3-phenylacrylonitrile (1k): colorless oil; Yield: $87 \%$; $384.9 \mathrm{mg}$<smiles>N#C/C=C(/Oc1ccccc1)c1ccccc1</smiles>
$(\mathrm{PE}: \mathrm{EA}=25: 1) ;{ }^{1} \mathrm{H}$ NMR $\left(\mathrm{CDCl}_{3}, 400 \mathrm{MHz}\right) \delta: .{ }^{1} \mathrm{H}$ NMR $\left(\mathrm{CDCl}_{3}, 400 \mathrm{MHz}\right) \delta:$ 7.99-7.97 (m, 2H), 7.54-7.46 (m, 5H), 7.44-7.28 (m, 1H), 7.26-7.12 (m, 2H), $4.55(\mathrm{~s}, 1 \mathrm{H}) .{ }^{13} \mathrm{C} \mathrm{NMR}$ $\left(\mathrm{CDCl}_{3}, 100 \mathrm{MHz}\right) \delta: 173.3,154.0,132.5,132.2,130.9,129.2$, $128.6,126.9,121.7,118.5,75.8 .^{[1]}$

(E)-3-(2-chlorophenoxy)-3-phenylacrylonitrile (11): colorless oil; Yield: $84 \%$;<smiles>N#C/C=C(/Oc1ccccc1)c1ccccc1</smiles>
$429.6 \mathrm{mg}$ (PE:EA $=20: 1) ;{ }^{1} \mathrm{H}$ NMR $\left(\mathrm{CDCl}_{3}, 400 \mathrm{MHz}\right) \delta$ : 7.90-7.88 (m, 2H), 7.43-7.36 (m, 3H), 7.25-7.21 (m, 1H), 7.16-7.12 (m, 2H), 7.07-7.05 (m, 1H), 4.28 (s, 1H). ${ }^{13} \mathrm{C}$ NMR 
$\left(\mathrm{CDCl}_{3}, 100 \mathrm{MHz}\right) \delta: 171.9,149.4,132.3,132.0,131.9,129.2,129.1,128.7,128.3$, 127.3, 123.8, 118.2, 75.4. TOF-HRMS Calcd. for $\mathrm{C}_{15} \mathrm{H}_{11} \mathrm{NOCl}\left[\mathrm{M}+\mathrm{H}^{+}\right]$: 256.0523, found 256.0523 .

(E)-3-phenyl-3-(p-tolyloxy)acrylonitrile (1m): colorless oil; Yield: $80 \%$; $376.4 \mathrm{mg}$<smiles>Cc1ccc(O/C(=C/C#N)c2ccccc2)cc1</smiles>
$(\mathrm{PE}: \mathrm{EA}=30: 1) ;{ }^{1} \mathrm{H} \mathrm{NMR}\left(\mathrm{CDCl}_{3}, 400 \mathrm{MHz}\right) \delta 7.98(\mathrm{dd}, J=$ 6.8, 1.4 Hz, 2H), 7.55-7.47 (m, 3H), 7.28-7.20 (m, 2H), 7.05-6.98 (m, 2H), $4.54(\mathrm{~s}, 1 \mathrm{H}), 2.37(\mathrm{~s}, 3 \mathrm{H}) .{ }^{13} \mathrm{C}$ NMR $\left(\mathrm{CDCl}_{3}, 100 \mathrm{MHz}\right) \delta: 173.1,151.3,136.3,132.2,131.6,130.9,128.7,128.15,120.9$, $118.2,74.8,20.9 .^{[1]}$

(Z)-3-methoxy-3-phenylacrylonitrile (1n): colorless oil; Yield: 95\%; $302.4 \mathrm{mg}$ $(\mathrm{PE}: \mathrm{EA}=3: 1) ;{ }^{1} \mathrm{H} \mathrm{NMR}\left(\mathrm{CDCl}_{3}, 400 \mathrm{MHz}\right) \delta: 7.53-7.36(\mathrm{~m}, 5 \mathrm{H})$,
$4.94(\mathrm{~s}, 1 \mathrm{H}), 4.24(\mathrm{~s}, 3 \mathrm{H}) .{ }^{13} \mathrm{C} \mathrm{NMR}\left(\mathrm{CDCl}_{3}, 100 \mathrm{MHz}\right) \delta: 171.5$,
$134.5,131.6,129.2,127.1,118.0,71.3,60.1 . \mathrm{TOF}-\mathrm{HRMS}$ Calcd. for
$\mathrm{C}_{10} \mathrm{H}_{10} \mathrm{NO}\left[\mathrm{M}+\mathrm{H}^{+}\right]: 160.0756$, found 160.0759. (Z)-3-isopropoxy-3-phenylacrylonitrile (10): colorless oil; Yield: 96\%; $359.4 \mathrm{mg}$ ${ }_{1}^{C N}$ $(\mathrm{PE}: \mathrm{EA}=3: 1) ;{ }^{1} \mathrm{H} \mathrm{NMR}\left(\mathrm{CDCl}_{3}, 400 \mathrm{MHz}\right) \delta:$ 7.47-7.22 (m, 5H), 4.90 $(\mathrm{s}, 1 \mathrm{H}), 4.89-4.84(\mathrm{~m}, 5 \mathrm{H}), 1.35(\mathrm{~s}, 3 \mathrm{H}), 1.34(\mathrm{~s}, 3 \mathrm{H}) .{ }^{13} \mathrm{C} \mathrm{NMR}\left(\mathrm{CDCl}_{3}\right.$, $100 \mathrm{MHz}) \delta: 170.2,134.3,131.1,128.7,126.8,117.3,74.9,74.4,22.3$. TOF-HRMS Calcd. for $\mathrm{C}_{12} \mathrm{H}_{14} \mathrm{NO}\left[\mathrm{M}+\mathrm{H}^{+}\right]$: 188.1069, found 188.1068.

(Z)-3-phenyl-3-(2,2,2-trifluoroethoxy)acrylonitrile (1p): colorless oil; Yield: 94\%; $\mathrm{F}_{3} \mathrm{C}$ $427.1 \mathrm{mg}(\mathrm{PE}: \mathrm{EA}=5: 1) ;{ }^{1} \mathrm{H} \mathrm{NMR}\left(\mathrm{CDCl}_{3}, 400 \mathrm{MHz}\right) \delta: 7.55-7.43$ (m, 5H), 5.14 (s, 1H), 4.69 (q, $J=7.9 \mathrm{~Hz}, 15.8 \mathrm{~Hz}, 2 \mathrm{H}) .{ }^{13} \mathrm{C} \mathrm{NMR}$ $\left(\mathrm{CDCl}_{3}, 100 \mathrm{MHz}\right) \delta: 170.3,132.5,129.7,127.4,121.7,116.3$, 76.9, 68.7 (q, $J=36.0 \mathrm{~Hz})$, 30.2. TOF-HRMS Calcd. for $\mathrm{C}_{11} \mathrm{H}_{9} \mathrm{NOF}_{3}\left[\mathrm{M}+\mathrm{H}^{+}\right]: 228.0630$, found 228.0632 .

(Z)-3-phenyl-3-(1H-pyrrol-1-yl)acrylonitrile (1q): White solid; MP: 62-64 ${ }^{\circ} \mathrm{C}$;<smiles>N#C/C(=C(\c1ccccc1)n1cccc1)c1ccccc1</smiles>
Yield: 89\%; $345.7 \mathrm{mg}(\mathrm{PE}: \mathrm{DCM}=5: 1) ;{ }^{1} \mathrm{H} \mathrm{NMR}\left(\mathrm{CDCl}_{3}, 400 \mathrm{MHz}\right)$ $\delta: 7.58-7.49(\mathrm{~m}, 5 \mathrm{H}), 6.76-6.75(\mathrm{~m}, 2 \mathrm{H}), 6.34-6.33(\mathrm{~m}, 2 \mathrm{H}), 5.39(\mathrm{~s}$, 1H). ${ }^{13} \mathrm{C} \mathrm{NMR}\left(\mathrm{CDCl}_{3}, 100 \mathrm{MHz}\right) \delta: 157.6,132.8,132.1,130.4$, 
$129.4,121.7,118.1,113.2,83.4,30.3 .^{[2]}$

(Z)-dimethyl (2-cyano-1-(p-tolyl)vinyl)phosphonate (1r): White solid; MP:<smiles>COP(=O)(OC)C(=CC#N)c1ccc(C)cc1</smiles>

68-70 ${ }^{\circ} \mathrm{C}$; Yield: 97\%; $487.4 \mathrm{mg}(\mathrm{PE}: \mathrm{EA}=1: 1) ;{ }^{1} \mathrm{H}$ NMR $\left(\mathrm{CDCl}_{3}, 400 \mathrm{MHz}\right) \delta:$ 7.47-7.44 (m, 2H), 7.26-7.24 (m, 2H), $6.40(\mathrm{~d}, J=20.2 \mathrm{~Hz}, 1 \mathrm{H}), 3.76(\mathrm{~s}, 3 \mathrm{H}), 3.74(\mathrm{~s}, 3 \mathrm{H})$,

$2.38(\mathrm{~s}, 3 \mathrm{H}) .{ }^{13} \mathrm{C} \mathrm{NMR}\left(\mathrm{CDCl}_{3}, 100 \mathrm{MHz}\right) \delta: 153.3,151.6,141.4,130.2,130.1,128.7$, 128.6, 116.0, 115.7, 111.2, 111.0, 54.0, 53.9, 22.0. TOF-HRMS Calcd. for $\mathrm{C}_{12} \mathrm{H}_{15} \mathrm{NO}_{3} \mathrm{P}\left[\mathrm{M}+\mathrm{H}^{+}\right]:$252.0784, found 252.0786.

(Z)-methyl 3-phenoxy-3-phenylacrylate (3a): colorless oil; Yield: 87\%; $442.4 \mathrm{mg}$<smiles>CC(=O)C=C(Oc1ccccc1)c1ccccc1</smiles>
$(\mathrm{PE}: \mathrm{EA}=8: 1) ;{ }^{1} \mathrm{H} \mathrm{NMR}\left(\mathrm{CDCl}_{3}, 400 \mathrm{MHz}\right) \delta: 7.61-7.58(\mathrm{~m}, 2 \mathrm{H})$, 7.37-7.33 (m, 3H), 7.26-7.24 (m, 2H), 6.98-6.96 (m, 3H), 6.14 (s, $1 \mathrm{H}), 3.68(\mathrm{~s}, 3 \mathrm{H}) .{ }^{13} \mathrm{C} \mathrm{NMR}\left(\mathrm{CDCl}_{3}, 100 \mathrm{MHz}\right) \delta: 165.4,162.6$, $157.5,134.6,131.2,130.1,129.3,127.7,122.9,116.9,106.4$, 51.9. TOF-HRMS Calcd. for $\mathrm{C}_{16} \mathrm{H}_{15} \mathrm{O}_{3}\left[\mathrm{M}+\mathrm{H}^{+}\right]$: 255.1015, found 255.1016.

(Z)-methyl 3-(2-methoxyphenoxy)-3-phenylacrylate (3b): colorless oil; Yield: 57\%;<smiles>COC(=O)/C=C(/Oc1ccccc1OC)c1ccccc1</smiles>
$324.1 \mathrm{mg}(\mathrm{PE}: \mathrm{EA}=8: 1) ;{ }^{1} \mathrm{H}$ NMR $\left(\mathrm{CDCl}_{3}, 400 \mathrm{MHz}\right) \delta$ : 7.59-7.56 (m, 2H), 7.36-7.28 (m, 3H), 6.95-6.88 (m, 2H), 6.79-6.71 (m, 2H), $6.01(\mathrm{~s}, 1 \mathrm{H}), 3.93(\mathrm{~s}, 3 \mathrm{H}), 3.68(\mathrm{~s}, 3 \mathrm{H}) .{ }^{13} \mathrm{C}$ $\operatorname{NMR}\left(\mathrm{CDCl}_{3}, 100 \mathrm{MHz}\right) \delta: 165.5,163.7,149.9,146.5,134.6$, 131.0, 129.1, 127.7, 123.8, 121.2, 117.6, 113.0, 105.5, 56.6, 51.9. TOF-HRMS Calcd. for $\mathrm{C}_{17} \mathrm{H}_{17} \mathrm{O}_{4}\left[\mathrm{M}+\mathrm{H}^{+}\right]: 285.1121$, found 285.1122.

(Z)-methyl 3-phenyl-3-(o-tolyloxy)acrylate (3c): colorless oil; Yield: 52\%; 279.0<smiles>CC(=O)C=C(Oc1ccccc1C)c1ccccc1</smiles>
mg (PE:EA = 10:1); ${ }^{1} \mathrm{H} \mathrm{NMR}\left(\mathrm{CDCl}_{3}, 400 \mathrm{MHz}\right) \delta: 7.59-7.56(\mathrm{~m}$, 2H), 7.38-7.33 (m, 3H), 7.20-7.18 (m, 1H), 6.96-6.86 (m, 2H), 6.62-6.60 (m, 1H), $6.12(\mathrm{~s}, 1 \mathrm{H}), 3.64(\mathrm{~s}, 3 \mathrm{H}), 2.47(\mathrm{~s}, 3 \mathrm{H}) .{ }^{13} \mathrm{C}$ NMR $\left(\mathrm{CDCl}_{3}, 100 \mathrm{MHz}\right) \delta: 165.4,163.2,155.9,134.8,131.6$, 131.2, 129.3, 127.5, 127.4, 127.2, 122.8, 114.8, 105.7, 51.8, 16.9. TOF-HRMS Calcd. for $\mathrm{C}_{17} \mathrm{H}_{17} \mathrm{O}_{3}\left[\mathrm{M}+\mathrm{H}^{+}\right]:$269.1172, found 269.1171.

(Z)-methyl 3-phenyl-3-(p-tolyloxy)acrylate (3d): colorless oil; Yield: 50\%; 268.3 mg (PE:EA = 8:1); ${ }^{1} \mathrm{H}$ NMR $\left(\mathrm{CDCl}_{3}, 400 \mathrm{MHz}\right)$ 8: 7.60-7.58 (m, 2H), 7.36-7.33 (m, 
3H), 7.03-7.01 (m, 2H), 6.88-6.86 (m, 2H), $6.11(\mathrm{~s}, 1 \mathrm{H}), 3.69(\mathrm{~s}, 3 \mathrm{H}), 2.24(\mathrm{~s}, 3 \mathrm{H})$.<smiles>COC(=O)C=C(Oc1ccc(C)cc1)c1ccccc1</smiles>
${ }^{13} \mathrm{C} \mathrm{NMR}\left(\mathrm{CDCl}_{3}, 100 \mathrm{MHz}\right) \delta: 165.5,162.9,155.4,134.7$, $132.3,131.1,130.5,129.3,127.8,116.8,106.2,51.9,21.1$. TOF-HRMS Calcd. for $\mathrm{C}_{17} \mathrm{H}_{17} \mathrm{O}_{3}\left[\mathrm{M}+\mathrm{H}^{+}\right]:$269.1172, found 269.1171.

(E)-methyl 3-phenyl-3-(p-tolyloxy)acrylate (3e): colorless oil; Yield: 47\%; 252.2<smiles>CC(=O)C=C(Oc1ccc(C)cc1)c1ccccc1</smiles>
mg (PE:EA = 10:1); ${ }^{1} \mathrm{H}$ NMR $\left(\mathrm{CDCl}_{3}, 400 \mathrm{MHz}\right) \delta$ : 7.65-7.63 (m, 2H), 7.47-7.42 (m, 3H), 7.26-7.20 (m, 2H), 7.03-7.01 (m, 2H), $5.17(\mathrm{~s}, 1 \mathrm{H}), 3.56(\mathrm{~s}, 3 \mathrm{H}), 2.36(\mathrm{~s}, 3 \mathrm{H})$.

${ }^{13} \mathrm{C}$ NMR $\left(\mathrm{CDCl}_{3}, 100 \mathrm{MHz}\right) \delta: 171.6,167.5,152.2,135.9$, 134.4, 131.1, 130.7, 129.8, 128.3, 121.6, 97.8, 51.6, 21.4. TOF-HRMS Calcd. for $\mathrm{C}_{17} \mathrm{H}_{17} \mathrm{O}_{3}\left[\mathrm{M}+\mathrm{H}^{+}\right]: 269.1172$, found 269.1171 .

(E)-methyl 3-(4-chlorophenoxy)-3-phenylacrylate (3f): colorless oil; Yield: 53\%;<smiles>CC(=O)C=C(Oc1ccc(Cl)cc1)c1ccccc1</smiles>
$306.1 \mathrm{mg}$ (PE:EA = 8:1); ${ }^{1} \mathrm{H} \mathrm{NMR}\left(\mathrm{CDCl}_{3}, 400 \mathrm{MHz}\right) \delta$ : 7.62-7.60 (m, 2H), 7.46-7.43 (m, 3H), 7.38-7.36 (m, 2H), 7.08-7.05 (m, 2H), $5.21(\mathrm{~s}, 1 \mathrm{H}), 3.57(\mathrm{~s}, 3 \mathrm{H}) .{ }^{13} \mathrm{C} \mathrm{NMR}$ $\left(\mathrm{CDCl}_{3}, 100 \mathrm{MHz}\right) \delta: 170.5,167.2,153.1,133.7,131.4$, 130.9, 130.7, 129.8, 128.4, 123.1, 99.1, 51.7. TOF-HRMS Calcd. for $\mathrm{C}_{16} \mathrm{H}_{14} \mathrm{O}_{3} \mathrm{Cl}$ $\left[\mathrm{M}+\mathrm{H}^{+}\right]:$289.0625, found 289.0625 .

(E)-ethyl 3-phenoxybut-2-enoate (3g): colorless oil; Yield: 82\%; $338.2 \mathrm{mg}$ (PE:EA

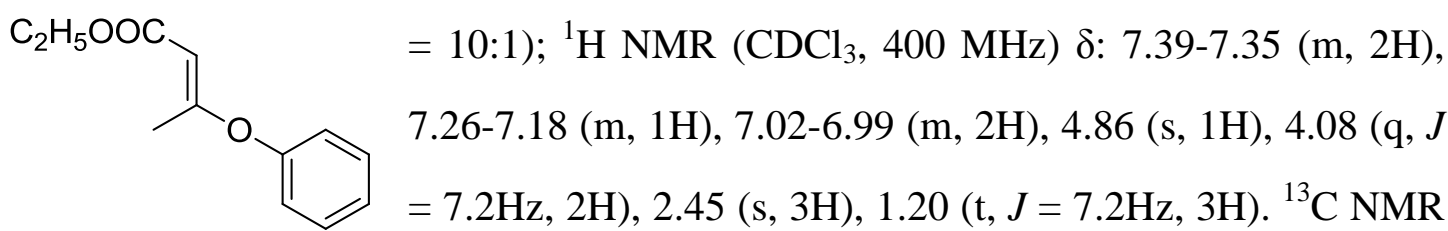
$\left(\mathrm{CDCl}_{3}, 100 \mathrm{MHz}\right) \delta: 173.2,168.2,153.9,130.5,126.2,122.1,96.7,60.0,18.9$, $14.8 .^{[6]}$

\section{Characterization data for compounds 2 and 4.}

3-phenoxy-3-phenylpropanenitrile (2a): White solid; MP: $101-103{ }^{\circ} \mathrm{C}$; Yield: $99 \%$; $22.1 \mathrm{mg}(\mathrm{PE}: \mathrm{EA}=10: 1) ; 99.9 \% \mathrm{ee} ;[\alpha]_{\mathrm{D}}^{25}=-62.4\left(\mathrm{c}=0.5, \mathrm{CH}_{2} \mathrm{Cl}_{2}\right)$; HPLC condition: Lux 5u Cellulose-1 $(250 \times 4.60 \mathrm{~mm})$, ipa:hex $=20: 80,1.0 \mathrm{~mL} / \mathrm{min}, 254 \mathrm{~nm} ; \mathrm{t}_{\mathrm{A}}=12.5$ $\min$ (major), $\mathrm{t}_{\mathrm{B}}=20.6 \mathrm{~min}$ (minor); ${ }^{1} \mathrm{H} \mathrm{NMR}\left(\mathrm{CDCl}_{3}, 400 \mathrm{MHz}\right) \delta: 7.44-7.34(\mathrm{~m}, 5 \mathrm{H})$, 
7.26-7.19 (m, 2H), 6.96-6.86 (m, 3H), $5.40(\mathrm{~d}, J=5.9 \mathrm{~Hz}, 1 \mathrm{H}), 3.01-2.88(\mathrm{~m}, 2 \mathrm{H}) .{ }^{13} \mathrm{C}$ 125.9, $122.0,116.7,116.2,75.5,27.6$. TOF-HRMS Calcd. for
$\mathrm{C}_{15} \mathrm{H}_{14} \mathrm{NO}\left[\mathrm{M}+\mathrm{H}^{+}\right]: 224.1069$, found 224.1071.

3-phenyl-3-(o-tolyloxy)propanenitrile (2b): White solid; MP: $61-63{ }^{\circ} \mathrm{C}$; Yield: $99 \%$; $\left.\mathrm{CH}_{2} \mathrm{Cl}_{2}\right) ; \mathrm{HPLC}$ condition: Lux 5u Cellulose-1 $(250 \times 4.60 \mathrm{~mm})$,
$\mathrm{ipa}: \mathrm{hex}=30: 70,1.0 \mathrm{~mL} / \mathrm{min}, 254 \mathrm{~nm} ; \mathrm{t}_{\mathrm{A}}=12.6 \mathrm{~min}$ (major), $\mathrm{t}_{\mathrm{B}}=$ 23.7 min (minor); ${ }^{1} \mathrm{H} \mathrm{NMR}\left(\mathrm{CDCl}_{3}, 400 \mathrm{MHz}\right) \delta: 7.35-7.33(\mathrm{~m}$, 5H), 7.11-7.09 (m, 1H), 6.95-6.91 (m, 1H), 6.81-6.77 (m, 1H), 6.53-6.51 (m, 1H), 5.37-5.34 (m, 1H), 2.96-2.84(m, 2H), $2.31(\mathrm{~s}, 3 \mathrm{H}) .{ }^{13} \mathrm{C} \mathrm{NMR}\left(\mathrm{CDCl}_{3}, 100 \mathrm{MHz}\right) \delta$ : $155.5,139.1,131.6,129.7,129.4,128.1,127.2$, 126.3, 122.0, 117.2, 113.4, 75.7, 28.0, $17.0 .^{[7]}$

3-(2-methoxyphenoxy)-3-phenylpropanenitrile (2c): White solid; MP: $97-99{ }^{\circ} \mathrm{C}$;<smiles>COc1ccccc1OC(=CCC#N)c1ccccc1</smiles>
Yield: 98\%; $24.8 \mathrm{mg}(\mathrm{PE}: \mathrm{EA}=10: 1) ; 99.9 \%$ ee; $[\alpha]_{\mathrm{D}}{ }^{25}=-49.4(\mathrm{c}=$ 0.5, $\left.\mathrm{CH}_{2} \mathrm{Cl}_{2}\right)$; HPLC condition: Lux 5u Cellulose-1 $(250 \times 4.60$ $\mathrm{mm}$ ), ipa:hex =30:70, $1.0 \mathrm{~mL} / \mathrm{min}, 254 \mathrm{~nm} ; \mathrm{t}_{\mathrm{A}}=11.0 \mathrm{~min}$ (major), $\mathrm{t}_{\mathrm{B}}=14.3 \min$ (minor); ${ }^{1} \mathrm{H} \mathrm{NMR}\left(\mathrm{CDCl}_{3}, 400 \mathrm{MHz}\right) \delta: 7.40-7.27(\mathrm{~m}$, $5 \mathrm{H}), 6.89-6.83(\mathrm{~m}, 2 \mathrm{H}), 6.70-6.69(\mathrm{~m}, 2 \mathrm{H}), 5.34-5.31(\mathrm{~m}, 1 \mathrm{H}), 3.82(\mathrm{~s}, 3 \mathrm{H}), 3.02-2.84$ (m, 2H). ${ }^{13} \mathrm{C} \mathrm{NMR}\left(\mathrm{CDCl}_{3}, 100 \mathrm{MHz}\right) \delta: 151.3,146.8,139.0,129.4,126.7,123.9$, 121.3, 118.9, 117.3, 113.2, 56.6, 27.5. TOF-HRMS Calcd. for $\mathrm{C}_{16} \mathrm{H}_{15} \mathrm{NO}_{2} \mathrm{Na}\left[\mathrm{M}+\mathrm{Na}^{+}\right]$: 276.0995, found 276.0994.

3-(2-chlorophenoxy)-3-phenylpropanenitrile (2d): White solid; MP: 93-95 ${ }^{\circ} \mathrm{C}$; $\left.0.5, \mathrm{CH}_{2} \mathrm{Cl}_{2}\right) ; \mathrm{HPLC}$ condition: Lux $5 \mathrm{u}$ Cellulose-1 $(250 \times 4.60$
$\mathrm{mm}), \mathrm{ipa}: \mathrm{hex}=30: 70,1.0 \mathrm{~mL} / \mathrm{min}, 254 \mathrm{~nm} ; \mathrm{t}_{\mathrm{A}}=13.0 \mathrm{~min}(\operatorname{minor})$,
$\mathrm{t}_{\mathrm{B}}=21.8 \mathrm{~min}$ (major); ${ }^{1} \mathrm{H} \mathrm{NMR}\left(\mathrm{CDCl}_{3}, 400 \mathrm{MHz}\right) \delta: 7.38-7.28(\mathrm{~m}$, $6 \mathrm{H}), 7.00-6.95(\mathrm{~m}, 1 \mathrm{H}), 6.83-6.80(\mathrm{~m}, 1 \mathrm{H}), 6.67-6.65(\mathrm{~m}, 1 \mathrm{H}), 5.37(\mathrm{~d}, J=6.2 \mathrm{~Hz}$, 1H), 3.04-2.88 (m, 2H). ${ }^{13} \mathrm{C} \mathrm{NMR}\left(\mathrm{CDCl}_{3}, 100 \mathrm{MHz}\right) \delta: 153.0,138.2,131.1,129.8$, 
129.7, 128.1, 126.5, 124.6, 123.3, 116.9, 116.7, 77.2, 27.8. TOF-HRMS Calcd. for $\mathrm{C}_{15} \mathrm{H}_{13} \mathrm{NOCl}\left[\mathrm{M}+\mathrm{H}^{+}\right]: 258.068$, found 258.0677.

3-(3-chlorophenoxy)-3-phenylpropanenitrile (2e): colorless oil; Yield: 97\%; 23.7<smiles></smiles>
$\operatorname{mg}(\mathrm{PE}: \mathrm{EA}=10: 1) ; 99.9 \%$ ee; $[\alpha]_{\mathrm{D}}{ }^{25}=-50.8\left(\mathrm{c}=0.5, \mathrm{CH}_{2} \mathrm{Cl}_{2}\right)$; HPLC condition: Lux 5u Cellulose-1 $(250 \times 4.60 \mathrm{~mm})$, ipa:hex $=30: 70,1.0 \mathrm{~mL} / \mathrm{min}, 254 \mathrm{~nm} ; \mathrm{t}_{\mathrm{A}}=11.4 \min ($ minor $), \mathrm{t}_{\mathrm{B}}=20.3$ $\min$ (major); ${ }^{1} \mathrm{H} \mathrm{NMR}\left(\mathrm{CDCl}_{3}, 400 \mathrm{MHz}\right) \delta: 7.41-7.37$ (m, 5H), 7.14-7.10 (m, 1H), 6.93-6.88 (m, 2H), 6.75-6.72 (m, 1H), 5.39-5.36 (m, 1H), 3.01-2.88 (m, 2H). ${ }^{13} \mathrm{C} \mathrm{NMR}\left(\mathrm{CDCl}_{3}, 100 \mathrm{MHz}\right) \delta: 158.1,138.2,135.4,130.8,129.8$, $129.7,126.3,122.7,117.3,116.9,114.8,76.2,28.0$. TOF-HRMS Calcd. for $\mathrm{C}_{15} \mathrm{H}_{13} \mathrm{NOCl}\left[\mathrm{M}+\mathrm{H}^{+}\right]: 258.068$, found 258.0677.

3-phenyl-3-(p-tolyloxy)propanenitrile (2f): White solid; MP: $96-98{ }^{\circ} \mathrm{C}$; Yield: $99 \%$;<smiles>Cc1ccc(OC(=CCC#N)c2ccccc2)cc1</smiles>
$23.4 \mathrm{mg}(\mathrm{PE}: \mathrm{EA}=10: 1) ; 99.9 \%$ ee; $[\alpha]_{\mathrm{D}}^{25}=-56.8(\mathrm{c}=0.5$, $\left.\mathrm{CH}_{2} \mathrm{Cl}_{2}\right)$; HPLC condition: Lux 5u Cellulose-1 $(250 \times 4.60$ $\mathrm{mm}$ ), ipa:hex $=20: 80,1.0 \mathrm{~mL} / \mathrm{min}, 254 \mathrm{~nm} ; \mathrm{t}_{\mathrm{A}}=13.5 \mathrm{~min}$ (major), $\mathrm{t}_{\mathrm{B}}=14.4 \mathrm{~min}$ (minor); ${ }^{1} \mathrm{H} \mathrm{NMR}\left(\mathrm{CDCl}_{3}, 400 \mathrm{MHz}\right) \delta$ : 7.35-7.32 (m, 5H), 6.96-6.94 (m, 2H), 6.72-6.69 (m, 2H), 5.30-5.27 (m, 1H), 2.93-2.80 (m, 1H), 2.18 (s, 3H). ${ }^{13} \mathrm{C}$ NMR $\left(\mathrm{CDCl}_{3}, 100 \mathrm{MHz}\right) \delta: 155.4,139.1,131.8$, 130.5, 129.6, 129.4, 126.4, 117.3, 116.6, 76.1, 28.0, 21.0. TOF-HRMS Calcd. for $\mathrm{C}_{16} \mathrm{H}_{15} \mathrm{NONa}\left[\mathrm{M}+\mathrm{Na}^{+}\right]: 260.1045$, found 260.1044.

3-(4-chlorophenoxy)-3-phenylpropanenitrile (2g): colorless oil; Yield: 99\%; 25.5<smiles>N#CCC(=O)c1ccccc1</smiles>
$\operatorname{mg}(\mathrm{PE}: \mathrm{EA}=12: 1) ; 99.7 \%$ ee; $[\alpha]_{\mathrm{D}}^{25}=-57.2(\mathrm{c}=0.5$, $\left.\mathrm{CH}_{2} \mathrm{Cl}_{2}\right)$; HPLC condition: Lux 5u Cellulose-1 $(250 \times 4.60$ $\mathrm{mm}$ ), ipa:hex $=30: 70,1.0 \mathrm{~mL} / \mathrm{min}, 254 \mathrm{~nm} ; \mathrm{t}_{\mathrm{A}}=12.2 \mathrm{~min}$ (minor), $\mathrm{t}_{\mathrm{B}}=13.7 \mathrm{~min}$ (major); ${ }^{1} \mathrm{H} \mathrm{NMR}\left(\mathrm{CDCl}_{3}, 400 \mathrm{MHz}\right) \delta$ : 7.39-7.37 (m, 5H), 7.16-7.14 (m, 2H), 6.79-6.77 (m, 2H), 5.36-5.33 (m, 1H), 3.00-2.88 (m, 2H). ${ }^{13} \mathrm{C} \mathrm{NMR}\left(\mathrm{CDCl}_{3}, 100 \mathrm{MHz}\right) \delta: 156.0,138.4,130.0,129.8,129.7$, 127.4, 126.3, 118.0, 117.0, 76.4, 28.0. TOF-HRMS Calcd. for $\mathrm{C}_{15} \mathrm{H}_{13} \mathrm{NOCl}\left[\mathrm{M}+\mathrm{H}^{+}\right]$: 258.068, found 258.0677. 
3-phenyl-3-(4-(trifluoromethyl)phenoxy)propanenitrile (2h): White solid; MP:<smiles>N#CCC(=O)c1ccccc1</smiles>
86-88 ${ }^{\circ} \mathrm{C}$; Yield: 98\%; $28.5 \mathrm{mg}(\mathrm{PE}: \mathrm{EA}=10: 1)$; 99.9\% ee; $[\alpha]_{\mathrm{D}}^{25}=+48.4\left(\mathrm{c}=0.5, \mathrm{CH}_{2} \mathrm{Cl}_{2}\right)$; HPLC condition: Lux $5 \mathrm{u}$ Cellulose-1 $(250 \times 4.60 \mathrm{~mm})$, ipa:hex $=30: 70,1.0 \mathrm{~mL} / \mathrm{min}$, $254 \mathrm{~nm} ; \mathrm{t}_{\mathrm{A}}=9.6 \min \left(\right.$ minor), $\mathrm{t}_{\mathrm{B}}=13.8 \min \left(\right.$ major) $;{ }^{1} \mathrm{H} \mathrm{NMR}$ $\left(\mathrm{CDCl}_{3}, 400 \mathrm{MHz}\right) \delta$ : 7.41-7.19 (m, 7H), 6.86-6.84 (m, 2H), 5.39-5.36 (m, 1H), 2.97-2.88 (m, 2H). ${ }^{13} \mathrm{C} \mathrm{NMR}\left(\mathrm{CDCl}_{3}, 100 \mathrm{MHz}\right) \delta: 159.8,138.0,129.9,129.8,128.6$ $\left(\mathrm{q},{ }^{1} J_{C-F}=230.0 \mathrm{~Hz}\right), 127.5,127.4,126.2,116.8,116.5,76.1,28.1 .^{[7]}$

3-(naphthalen-1-yloxy)-3-phenylpropanenitrile (2i): White solid; MP: $98-100{ }^{\circ} \mathrm{C}$;<smiles>N#CCC(=O)c1ccccc1</smiles>
Yield: 99\%; $27.1 \mathrm{mg}(\mathrm{PE}: \mathrm{EA}=10: 1) ; 99.9 \%$ ee; $[\alpha]_{\mathrm{D}}{ }^{25}=+82.8$ $\left(\mathrm{c}=0.5, \mathrm{CH}_{2} \mathrm{Cl}_{2}\right)$; HPLC condition: Lux 5u Cellulose-1 $(250 \times$ $4.60 \mathrm{~mm}$ ), ipa:hex =10:90,1.0 mL/min, $254 \mathrm{~nm} ; \mathrm{t}_{\mathrm{A}}=11.6 \mathrm{~min}$ (minor), $\mathrm{t}_{\mathrm{B}}=28.1 \mathrm{~min}$ (major); ${ }^{1} \mathrm{H} \mathrm{NMR}\left(\mathrm{CDCl}_{3}, 400 \mathrm{MHz}\right) \delta$ : 8.41-8.39 (m, 1H), 7.75-7.73 (m, 1H), 7.51-7.28 (m, 8H), 7.16-7.12 (m, 1H), $6.53(\mathrm{~d}$, $J=7.6 \mathrm{~Hz}, 1 \mathrm{H}), 5.55(\mathrm{t}, J=5.9 \mathrm{~Hz}, 1 \mathrm{H}), 3.07-2.97(\mathrm{~m}, 2 \mathrm{H}) .{ }^{13} \mathrm{C} \mathrm{NMR}\left(\mathrm{CDCl}_{3}, 100\right.$ MHz) $\delta: 152.8,138.7,135.1,129.7,129.6,128.1,127.2,126.3,126.2,126.0,122.5$, 121.9, 117.1, 107.7, 75.8, 28.1. TOF-HRMS Calcd. for $\mathrm{C}_{19} \mathrm{H}_{16} \mathrm{NO}\left[\mathrm{M}+\mathrm{H}^{+}\right]$: 274.1226, found 274.1227 .

3-(naphthalen-2-yloxy)-3-phenylpropanenitrile (2j): White solid; MP: 105-107 ${ }^{\circ} \mathrm{C}$;<smiles>N#CCC(=O)c1ccccc1</smiles>
Yield: 98\%; $26.9 \mathrm{mg}(\mathrm{PE}: \mathrm{EA}=10: 1) ; 99.6 \%$ ee; $[\alpha]_{\mathrm{D}}{ }^{25}=$ $-55.8\left(\mathrm{c}=0.5, \mathrm{CH}_{2} \mathrm{Cl}_{2}\right)$; HPLC condition: Lux 5u Cellulose-1 $(250 \times 4.60 \mathrm{~mm})$, ipa:hex $=30: 70,1.0 \mathrm{~mL} / \mathrm{min}, 254 \mathrm{~nm} ; \mathrm{t}_{\mathrm{A}}=$ $12.6 \min$ (minor), $\mathrm{t}_{\mathrm{B}}=21.9 \min$ (major); ${ }^{1} \mathrm{H} \mathrm{NMR}\left(\mathrm{CDCl}_{3}\right.$, $400 \mathrm{MHz}) \delta:$ 7.69-7.66 (m, 2H), 7.54-7.52 (m, 1H), 7.42-7.14 (m, 8H), $6.95(\mathrm{~s}, 1 \mathrm{H})$, 5.50-5.47 (m, 1H), 3.00-2.88 (m, 2H). ${ }^{13} \mathrm{C} \mathrm{NMR}\left(\mathrm{CDCl}_{3}, 100 \mathrm{MHz}\right) \delta: 155.3,138.7$, $134.6,130.2,129.9,129.7,129.5,128.2,127.4,127.0,126.4,124.7,119.5,117.1$, 110.3, 76.0, 28.0. TOF-HRMS Calcd. for $\mathrm{C}_{19} \mathrm{H}_{16} \mathrm{NO}\left[\mathrm{M}+\mathrm{H}^{+}\right]$: 274.1226, found 274.1227.

3-phenoxy-3-phenylpropanenitrile (2k): White solid; MP: $101-103{ }^{\circ} \mathrm{C}$; Yield: $98 \%$; $21.8 \mathrm{mg}(\mathrm{PE}: \mathrm{EA}=10: 1) ; 99.8 \% \mathrm{ee} ;[\alpha]_{\mathrm{D}}^{25}=+60.2\left(\mathrm{c}=0.5, \mathrm{CH}_{2} \mathrm{Cl}_{2}\right) ;$ HPLC condition: 
Lux 5u Cellulose-1 $(250 \times 4.60 \mathrm{~mm})$, ipa:hex $=20: 80,1.0 \mathrm{~mL} / \mathrm{min}, 254 \mathrm{~nm} ; \mathrm{t}_{\mathrm{A}}=11.8$

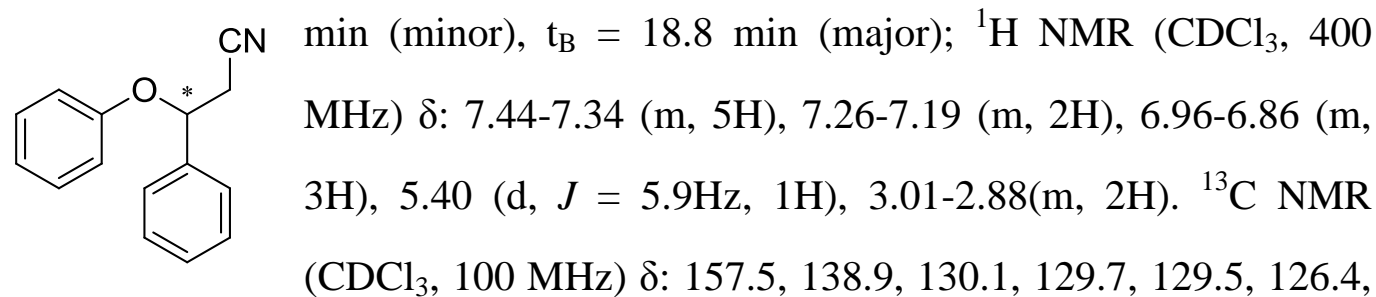

122.4, 117.2, 116.7, 75.9, 28.0. TOF-HRMS Calcd. for $\mathrm{C}_{15} \mathrm{H}_{14} \mathrm{NO}\left[\mathrm{M}+\mathrm{H}^{+}\right]$: 224.1069, found 224.1071 .

3-(2-chlorophenoxy)-3-phenylpropanenitrile (2l): White solid; MP: 93-95 ${ }^{\circ} \mathrm{C}$; Yield:<smiles>N#CCC(=O)c1ccccc1</smiles>
$97 \% ; 23.7 \mathrm{mg}(\mathrm{PE}: \mathrm{EA}=10: 1) ; 99.6 \%$ ee; $[\alpha]_{\mathrm{D}}{ }^{25}=-58.6(\mathrm{c}=0.5$, $\left.\mathrm{CH}_{2} \mathrm{Cl}_{2}\right)$; HPLC condition: Lux 5u Cellulose-1 $(250 \times 4.60 \mathrm{~mm})$, ipa:hex $=30: 70,1.0 \mathrm{~mL} / \mathrm{min}, 254 \mathrm{~nm} ; \mathrm{t}_{\mathrm{A}}=12.9 \min$ (major), $\mathrm{t}_{\mathrm{B}}=$ 20.9 min (minor); ${ }^{1} \mathrm{H}$ NMR $\left(\mathrm{CDCl}_{3}, 400 \mathrm{MHz}\right)$ 8: 7.38-7.28 (m, 6H), 7.00-6.95 (m, 1H), 6.83-6.80 (m, 1H), 6.67-6.65 (m, 1H), $5.37(\mathrm{~d}, J=6.2 \mathrm{~Hz}$, 1H), 3.04-2.88 (m, 2H). ${ }^{13} \mathrm{C} \mathrm{NMR}\left(\mathrm{CDCl}_{3}, 100 \mathrm{MHz}\right) \delta: 153.0,138.2,131.1,129.8$, 129.7, 128.1, 126.5, 124.6, 123.3, 116.9, 116.7, 77.2, 27.8. TOF-HRMS Calcd. for $\mathrm{C}_{15} \mathrm{H}_{13} \mathrm{NOCl}\left[\mathrm{M}+\mathrm{H}^{+}\right]: 258.068$, found 258.0677.

3-phenyl-3-(p-tolyloxy)propanenitrile (2m): White solid; MP: $96-98{ }^{\circ} \mathrm{C}$; Yield: $99 \%$;<smiles>Cc1ccc(OC(=CCC#N)c2ccccc2)cc1</smiles>

$23.5 \mathrm{mg}(\mathrm{PE}: \mathrm{EA}=10: 1) ; 99.1 \%$ ee; $[\alpha]_{\mathrm{D}}^{25}=+55.2(\mathrm{c}=0.5$, $\left.\mathrm{CH}_{2} \mathrm{Cl}_{2}\right)$; HPLC condition: Lux 5u Cellulose-1 $(250 \times 4.60$ $\mathrm{mm}$ ), ipa:hex $=20: 80,1.0 \mathrm{~mL} / \mathrm{min}, 254 \mathrm{~nm} ; \mathrm{t}_{\mathrm{A}}=12.1 \mathrm{~min}$ (minor), $\mathrm{t}_{\mathrm{B}}=13.0 \mathrm{~min}$ (major); ${ }^{1} \mathrm{H} \mathrm{NMR}\left(\mathrm{CDCl}_{3}, 400 \mathrm{MHz}\right) \delta$ : 7.35-7.32 (m, 5H), 6.96-6.94 (m, 2H), 6.72-6.69 (m, 2H), 5.30-5.27 (m, 1H), 2.93-2.80 (m, 1H), 2.18 (s, 3H). ${ }^{13} \mathrm{C}$ NMR $\left(\mathrm{CDCl}_{3}, 100 \mathrm{MHz}\right) \delta: 155.4,139.1,131.8$, 130.5, 129.6, 129.4, 126.4, 117.3, 116.6, 76.1, 28.0, 21.0. TOF-HRMS Calcd. for $\mathrm{C}_{16} \mathrm{H}_{15} \mathrm{NONa}\left[\mathrm{M}+\mathrm{Na}^{+}\right]: 260.1045$, found 260.1044 .

3-methoxy-3-phenylpropanenitrile (2n): colorless oil; Yield: 96\%; 15.5 mg (PE:EA $\mathrm{CN}=3: 1) ; 94 \%$ ee; $[\alpha]_{\mathrm{D}}{ }^{25}=-88.4\left(\mathrm{c}=0.5, \mathrm{CH}_{2} \mathrm{Cl}_{2}\right) ; \mathrm{GC}$ condition: Supelco
$\mathrm{mL} / \mathrm{min}$, programmed $90{ }^{\circ} \mathrm{C}-1{ }^{\circ} \mathrm{C} / \mathrm{min}-200{ }^{\circ} \mathrm{C}-100 \mathrm{~min} ; \mathrm{t}_{\mathrm{A}}=48.2$ 
$\min ($ major $), \mathrm{t}_{\mathrm{B}}=50.3 \mathrm{~min}$ (minor); ${ }^{1} \mathrm{H} \mathrm{NMR}\left(\mathrm{CDCl}_{3}, 400 \mathrm{MHz}\right) \delta: 7.42-7.33(\mathrm{~m}, 5 \mathrm{H})$, 4.47-4.43 (m, 1H), 3.29 (s, 1H), 2.79-2.66 (m, 2H). ${ }^{13} \mathrm{C} \mathrm{NMR}\left(\mathrm{CDCl}_{3}, 100 \mathrm{MHz}\right) \delta$ : $139.1,129.5,129.4,126.9,117.8,79.4,57.6,27.5 .^{[8]}$

3-isopropoxy-3-phenylpropanenitrile (2o): colorless oil; Yield: 96\%; $18.2 \mathrm{mg}$<smiles>CC(C)OC(=O)c1ccccc1</smiles>
$(\mathrm{PE}: \mathrm{EA}=2: 1) ; 90 \%$ ee; $[\alpha]_{\mathrm{D}}^{25}=-72.0\left(\mathrm{c}=0.5, \mathrm{CH}_{2} \mathrm{Cl}_{2}\right) ; \mathrm{GC}$ condition: Supelco beta $\operatorname{Dex}^{\mathrm{TM}} 120$ column $(30 \mathrm{~m} \times 0.25 \mathrm{~mm} \times 0.25$ $\mu \mathrm{m}), \mathrm{N}_{2} 1.0 \mathrm{~mL} / \mathrm{min}$, programmed $90{ }^{\circ} \mathrm{C}-1{ }^{\circ} \mathrm{C} / \mathrm{min}-200{ }^{\circ} \mathrm{C}-100$ $\min ; \mathrm{t}_{\mathrm{A}}=54.9 \min$ (major), $\mathrm{t}_{\mathrm{B}}=55.5 \min$ (minor); ${ }^{1} \mathrm{H} \mathrm{NMR}\left(\mathrm{CDCl}_{3}\right.$, $400 \mathrm{MHz}) \delta:$ 7.41-7.33 (m, 5H), 4.69-4.65 (m, 1H), 3.60-3.54 (m, 1H), 2.74-2.60 (m, $2 \mathrm{H}), 1.21(\mathrm{~d}, J=6.0 \mathrm{~Hz}, 3 \mathrm{H}), 1.12(\mathrm{~d}, J=6.2 \mathrm{~Hz}, 3 \mathrm{H}) .{ }^{13} \mathrm{C} \mathrm{NMR}\left(\mathrm{CDCl}_{3}, 100 \mathrm{MHz}\right) \delta$ : 140.6, 129.3, 129.1, 126.7, 118.0, 75.0, 70.5, 28.1, 23.6, 21.6. TOF-HRMS Calcd. for $\mathrm{C}_{12} \mathrm{H}_{16} \mathrm{NO}\left[\mathrm{M}+\mathrm{H}^{+}\right]:$190.1226, found 190.1227.

3-phenyl-3-(2,2,2-trifluoroethoxy)propanenitrile (2p): colorless oil; Yield: 97\%; $22.2 \mathrm{mg}(\mathrm{PE}: \mathrm{EA}=3: 1) ; 96 \%$ ee; $[\alpha]_{\mathrm{D}}{ }^{25}=-83.6\left(\mathrm{c}=0.5, \mathrm{CH}_{2} \mathrm{Cl}_{2}\right)$;
HPLC condition: Lux $5 \mathrm{u}$ Cellulose-1 $(250 \times 4.60 \mathrm{~mm})$, ipa:hex =
$30: 70,1.0 \mathrm{~mL} / \mathrm{min}, 254 \mathrm{~nm} ; \mathrm{t}_{\mathrm{A}}=9.6 \mathrm{~min}($ major $), \mathrm{t}_{\mathrm{B}}=11.2 \mathrm{~min}$
$($ minor $) ;{ }^{1} \mathrm{H} \mathrm{NMR}\left(\mathrm{CDCl}_{3}, 400 \mathrm{MHz}\right) \delta: 7.44-7.26(\mathrm{~m}, 5 \mathrm{H}), 4.74(\mathrm{~d}$, $J=6.3 \mathrm{~Hz}, 1 \mathrm{H}), 3.82-3.65(\mathrm{~m}, 2 \mathrm{H}), 2.90-2.74(\mathrm{~m}, 2 \mathrm{H}) .{ }^{13} \mathrm{C} \mathrm{NMR}\left(\mathrm{CDCl}_{3}, 100 \mathrm{MHz}\right) \delta$ : $136.8,129.7,129.3,126.5,122.5,116.3,78.5,66.1(\mathrm{q}, J=34.0 \mathrm{~Hz}), 26.9$. TOF-HRMS Calcd. for $\mathrm{C}_{11} \mathrm{H}_{11} \mathrm{NOF}_{3}\left[\mathrm{M}+\mathrm{H}^{+}\right]$: 230.0787, found 230.0788.

3-phenyl-3-(1H-pyrrol-1-yl)propanenitrile (2q): White solid; MP: $66-68{ }^{\circ} \mathrm{C}$; Yield:<smiles>N#CCC(c1ccccc1)n1cccc1</smiles>
$98 \% ; 19.2 \mathrm{mg}(\mathrm{PE}: \mathrm{EA}=3: 1) ; 97 \%$ ee; $[\alpha]_{\mathrm{D}}{ }^{25}=+32.6(\mathrm{c}=0.5$, $\left.\mathrm{CH}_{2} \mathrm{Cl}_{2}\right)$; HPLC condition: Lux 5u Cellulose-2 $(250 \times 4.60 \mathrm{~mm})$, ipa:hex = 30:70, $1.0 \mathrm{~mL} / \mathrm{min}, 254 \mathrm{~nm} ; \mathrm{t}_{\mathrm{A}}=10.1 \mathrm{~min}$ (major), $\mathrm{t}_{\mathrm{B}}=12.9$ $\min$ (minor); ${ }^{1} \mathrm{H} \mathrm{NMR}\left(\mathrm{CDCl}_{3}, 400 \mathrm{MHz}\right) \delta$ : 7.27-7.22 (m, 3H), 7.08-7.06 (m, 2H), $6.63(\mathrm{~d}, J=2.1 \mathrm{~Hz}, 2 \mathrm{H}), 6.10(\mathrm{~d}, J=2.1 \mathrm{~Hz}, 2 \mathrm{H}), 5.34(\mathrm{~d}, J=$ 7.2Hz, 1H), 3.08-3.06 (m, 2H). ${ }^{13} \mathrm{C} \mathrm{NMR}\left(\mathrm{CDCl}_{3}, 100 \mathrm{MHz}\right) \delta: 138.1,129.2,128.9$, $126.3,119.6,116.7,109.4,58.9,24.9 .^{[9]}$

dimethyl (2-cyano-1-(p-tolyl)ethyl)phosphonate (2r): colorless oil; Yield: 99\%; $25.1 \mathrm{mg}(\mathrm{PE}: \mathrm{EA}=2: 1) ; 99.5 \% \mathrm{ee} ;[\alpha]_{\mathrm{D}}^{25}=-84.8\left(\mathrm{c}=0.5, \mathrm{CH}_{2} \mathrm{Cl}_{2}\right)$; HPLC condition: 
Lux 5u Cellulose-2 $(250 \times 4.60 \mathrm{~mm})$, ipa:hex $=30: 70,1.0 \mathrm{~mL} / \mathrm{min}, 230 \mathrm{~nm} ; \mathrm{t}_{\mathrm{A}}=14.2$<smiles>COP(=O)(OC)[C@H](CC#N)c1ccc(C)cc1</smiles>
$\min$ (minor), $\mathrm{t}_{\mathrm{B}}=15.7$ min (major); ${ }^{1} \mathrm{H} \mathrm{NMR}\left(\mathrm{CDCl}_{3}, 400\right.$

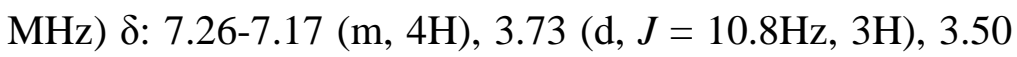
(d, $J=10.6 \mathrm{~Hz}, 3 \mathrm{H}), 3.40-3.31(\mathrm{~m}, 1 \mathrm{H}), 3.10-2.92(\mathrm{~m}, 2 \mathrm{H})$, $2.34(\mathrm{~s}, 3 \mathrm{H}) .{ }^{13} \mathrm{C} \mathrm{NMR}\left(\mathrm{CDCl}_{3}, 100 \mathrm{MHz}\right) \delta: 138.9,130.4,130.4,130.3,130.2,129.2$, 129.1, 118.0, 117.8, 54.6, 54.5, 53.6, 53.5, 41.4, 40.0, 21.7, 20.2, 19.7. TOF-HRMS Calcd. for $\mathrm{C}_{12} \mathrm{H}_{17} \mathrm{NO}_{3} \mathrm{P}\left[\mathrm{M}+\mathrm{H}^{+}\right]$: 254.0940, found 254.0939.

methyl 3-phenoxy-3-phenylpropanoate (4a): colorless oil; Yield: 97\%; $24.8 \mathrm{mg}$ (c) $(\mathrm{PE}: \mathrm{EA}=6: 1) ; 94 \%$ ee; $[\alpha]_{\mathrm{D}}{ }^{25}=+14.0\left(\mathrm{c}=0.5, \mathrm{CH}_{2} \mathrm{Cl}_{2}\right)$; HPLC condition: Lux 5u Cellulose-3 $(250 \times 4.60 \mathrm{~mm})$, ipa:hex $=$ $50: 50,1.0 \mathrm{~mL} / \mathrm{min}, 230 \mathrm{~nm} ; \mathrm{t}_{\mathrm{A}}=15.9 \mathrm{~min}$ (minor), $\mathrm{t}_{\mathrm{B}}=26.0 \mathrm{~min}$ (major); ${ }^{1} \mathrm{H}$ NMR $\left(\mathrm{CDCl}_{3}, 400 \mathrm{MHz}\right) \delta$ : 7.36-7.34 (m, 2H), 7.32-7.29 (m, 2H), 7.27-7.26 (m, 1H), 7.20-7.17 (m, 2H), 6.91-6.87 (m, 3H), 5.67-5.63 (m, 1H), $3.70(\mathrm{~s}, 3 \mathrm{H}), 3.10-3.04(\mathrm{~m}, 1 \mathrm{H}), 2.81-2.77(\mathrm{~m}, 1 \mathrm{H}) .{ }^{13} \mathrm{C} \mathrm{NMR}$ $\left(\mathrm{CDCl}_{3}, 100 \mathrm{MHz}\right) \delta: 171.4,158.3,140.9,129.9,129.3,128.6,126.6,121.8,116.8$, $52.4,44.2 .^{[5]}$

methyl 3-(2-methoxyphenoxy)-3-phenylpropanoate (4b): colorless oil; Yield: 96\%;<smiles>COC(=O)CC(Oc1ccccc1OC)c1ccccc1</smiles>
$27.5 \mathrm{mg}(\mathrm{PE}: \mathrm{EA}=8: 1) ; 99 \%$ ee; $[\alpha]_{\mathrm{D}}^{25}=+7.8\left(\mathrm{c}=0.5, \mathrm{CH}_{2} \mathrm{Cl}_{2}\right)$; HPLC condition: Lux 5u Cellulose-3 $(250 \times 4.60 \mathrm{~mm})$, ipa:hex $=$ $20: 80,1.0 \mathrm{~mL} / \mathrm{min}, 254 \mathrm{~nm} ; \mathrm{t}_{\mathrm{A}}=16.6 \mathrm{~min}$ (major), $\mathrm{t}_{\mathrm{B}}=22.8 \mathrm{~min}$ (minor); ${ }^{1} \mathrm{H}$ NMR $\left(\mathrm{CDCl}_{3}, 400 \mathrm{MHz}\right) \delta: 7.44-7.42(\mathrm{~m}, 2 \mathrm{H})$, 7.35-7.26 (m, 3H), 6.88-6.73 (m, 4H), 5.64-5.61 (m, 1H), $3.84(\mathrm{~s}, 3 \mathrm{H}), 3.67(\mathrm{~s}, 3 \mathrm{H})$, 3.21-3.15 (m, 1H), 2.85-2.80 (m, 1H). ${ }^{13} \mathrm{C} \mathrm{NMR}\left(\mathrm{CDCl}_{3}, 100 \mathrm{MHz}\right) \delta: 171.5,150.9$, $147.8,141.0,129.1,128.6,126.8,122.6,121.3,117.6,112.9,56.6,52.3,43.9 .^{[5]}$

methyl 3-phenyl-3-(o-tolyloxy)propanoate (4c): colorless oil; Yield: 96\%; $25.9 \mathrm{mg}$<smiles>CC(=O)CC(Oc1ccccc1C)c1ccccc1</smiles>
$(\mathrm{PE}: \mathrm{EA}=8: 1) ; 99.4 \%$ ee; $[\alpha]_{\mathrm{D}}^{25}=+9.8\left(\mathrm{c}=0.5, \mathrm{CH}_{2} \mathrm{Cl}_{2}\right)$; HPLC condition: Lux 5u Cellulose-1 $(250 \times 4.60 \mathrm{~mm})$, ipa:hex $=$ 20:80, $1.0 \mathrm{~mL} / \mathrm{min}, 254 \mathrm{~nm} ; \mathrm{t}_{\mathrm{A}}=5.3 \min$ (major), $\mathrm{t}_{\mathrm{B}}=7.5 \mathrm{~min}$ (minor); ${ }^{1} \mathrm{H} \mathrm{NMR}\left(\mathrm{CDCl}_{3}, 400 \mathrm{MHz}\right) \delta$ : 7.44-7.28(m, 5H), 7.14-7.12 (m, 1H), 7.02-6.98 (m, 1H), 6.83-6.80 (m, 1H), 6.71-6.69 (m, 1H), 
5.69-5.66 (m, 1H), $3.71(\mathrm{~s}, 3 \mathrm{H}), 3.14-3.08(\mathrm{~m}, 1 \mathrm{H}), 2.85-2.80(\mathrm{~m}, 1 \mathrm{H}), 2.30(\mathrm{~s}, 3 \mathrm{H})$. ${ }^{13} \mathrm{C} \mathrm{NMR}\left(\mathrm{CDCl}_{3}, 100 \mathrm{MHz}\right) \delta: 171.5,156.3,141.2,131.2,129.4,128.6,127.8,127.1$, $126.4,121.3,113.8,52.4,44.4,16.9 .^{[5]}$

methyl 3-phenyl-3-(p-tolyloxy)propanoate (4d, 4e): colorless oil; 4d: Yield: 97\%;<smiles>CC(=O)CC(Oc1ccc(C)cc1)c1ccccc1</smiles>
$26.2 \mathrm{mg}(\mathrm{PE}: \mathrm{EA}=8: 1) ; 93 \%$ ee; $[\alpha]_{\mathrm{D}}^{25}=+10.2(\mathrm{c}=0.5$, $\left.\mathrm{CH}_{2} \mathrm{Cl}_{2}\right)$; HPLC condition: Lux 5u Cellulose-1 $(250 \times 4.60$ $\mathrm{mm})$, ipa:hex $=30: 70,1.0 \mathrm{~mL} / \mathrm{min}, 230 \mathrm{~nm} ; \mathrm{t}_{\mathrm{A}}=4.7 \mathrm{~min}$ (major), $\mathrm{t}_{\mathrm{B}}=6.9 \min$ (minor); 4e: Yield: 98\%; $25.9 \mathrm{mg}$ $(\mathrm{PE}: \mathrm{EA}=8: 1) ; 96 \%$ ee; $[\alpha]_{\mathrm{D}}{ }^{25}=-12.4\left(\mathrm{c}=0.5, \mathrm{CH}_{2} \mathrm{Cl}_{2}\right)$; HPLC condition: Lux $5 \mathrm{u}$ Cellulose-1 $(250 \times 4.60 \mathrm{~mm})$, ipa:hex $=30: 70,1.0 \mathrm{~mL} / \mathrm{min}, 230 \mathrm{~nm} ; \mathrm{t}_{\mathrm{A}}=4.7 \mathrm{~min}$ (minor), $\mathrm{t}_{\mathrm{B}}=7.0 \mathrm{~min}$ (major); ${ }^{1} \mathrm{H} \mathrm{NMR}\left(\mathrm{CDCl}_{3}, 400 \mathrm{MHz}\right) \delta: 7.39-7.25(\mathrm{~m}, 5 \mathrm{H})$, 6.99-6.97 (m, 2H), 6.78-6.75 (m, 2H), 5.61-5.58 (m, 1H), $3.70(\mathrm{~s}, 3 \mathrm{H}), 3.07-3.01(\mathrm{~m}$, 1H), 2.80-2.75 (m, 1H), $2.22(\mathrm{~s}, 3 \mathrm{H}) .{ }^{13} \mathrm{C} \mathrm{NMR}\left(\mathrm{CDCl}_{3}, 100 \mathrm{MHz}\right) \delta: 171.5,156.1$, $141.1,131.0,130.3,129.3,128.5,126.6,116.8,52.4,44.2,21.0 .^{[5]}$

methyl 3-(4-chlorophenoxy)-3-phenylpropanoate (4f): colorless oil; Yield: 98\%;

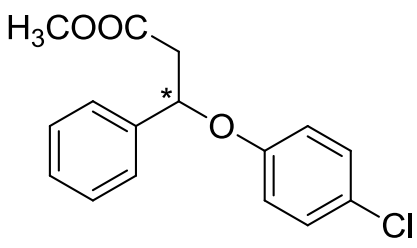
$28.5 \mathrm{mg}(\mathrm{PE}: \mathrm{EA}=6: 1) ; 97 \% \mathrm{ee} ;[\alpha]_{\mathrm{D}}^{25}=+6.8(\mathrm{c}=0.5$, $\left.\mathrm{CH}_{2} \mathrm{Cl}_{2}\right)$; HPLC condition: Lux 5u Cellulose-1 $(250 \times 4.60$ $\mathrm{mm})$, ipa:hex $=20: 80,1.0 \mathrm{~mL} / \mathrm{min}, 230 \mathrm{~nm} ; \mathrm{t}_{\mathrm{A}}=5.4 \mathrm{~min}$ (major), $\mathrm{t}_{\mathrm{B}}=8.9 \min ($ minor $) ;{ }^{1} \mathrm{H} \mathrm{NMR}\left(\mathrm{CDCl}_{3}, 400 \mathrm{MHz}\right)$ $\delta: 7.38-7.28(\mathrm{~m}, 5 \mathrm{H}), 7.13-7.11(\mathrm{~m}, 2 \mathrm{H}), 6.79-6.77(\mathrm{~m}, 2 \mathrm{H}), 5.59-5.56(\mathrm{~m}, 1 \mathrm{H}), 3.70(\mathrm{~s}$, $3 \mathrm{H}), 3.07-3.01(\mathrm{~m}, 1 \mathrm{H}), 2.79-2.74(\mathrm{~m}, 1 \mathrm{H}) .{ }^{13} \mathrm{C} \mathrm{NMR}\left(\mathrm{CDCl}_{3}, 100 \mathrm{MHz}\right) \delta: 171.3$, $156.8,140.4,129.8,129.4,128.8,126.7,126.5,118.2,52.4,44.0 .^{[5]}$

ethyl 3-phenoxybutanoate (4g): colorless oil; Yield: 98\%; $20.4 \mathrm{mg}(\mathrm{PE}: \mathrm{EA}=8: 1$ ); $\mathrm{C}_{2} \mathrm{H}_{5} \mathrm{OOC}$ $90 \%$ ee; $[\alpha]_{\mathrm{D}}^{25}=+10.8\left(\mathrm{c}=0.5, \mathrm{CH}_{2} \mathrm{Cl}_{2}\right)$; HPLC condition: Lux 5u Cellulose-1 $(250 \times 4.60 \mathrm{~mm})$, ipa:hex $=10: 90,1.0$ $\mathrm{mL} / \min , 254 \mathrm{~nm} ; \mathrm{t}_{\mathrm{A}}=4.2 \min \left(\right.$ minor), $\mathrm{t}_{\mathrm{B}}=4.6 \min$ (major); ${ }^{1} \mathrm{H}$ NMR $\left(\mathrm{CDCl}_{3}, 400 \mathrm{MHz}\right)$ 8: 7.29-7.25(m, 2H), 6.97-6.92 (m, 3H), 4.88-4.80 (m, 1H), 4.14 (q, $J=14.3 \mathrm{~Hz}, 7.1 \mathrm{~Hz}, 2 \mathrm{H}), 2.83-2.78(\mathrm{~m}, 1 \mathrm{H})$, 2.55-2.50 (m, 1H), $1.37(\mathrm{~d}, J=6.1 \mathrm{~Hz}, 3 \mathrm{H}), 1.24(\mathrm{t}, J=7.1 \mathrm{~Hz}, 3 \mathrm{H}) .{ }^{13} \mathrm{C} \mathrm{NMR}\left(\mathrm{CDCl}_{3}\right.$, $100 \mathrm{MHz}) \delta$ : $171.5,158.0,130.1,121.7,116.8,71.3,61.1,42.2,20.4,14.7 .^{[6]}$ 
6. Procedure for the synthesis of $(S)$-Nisoxetine ${ }^{[10]}$
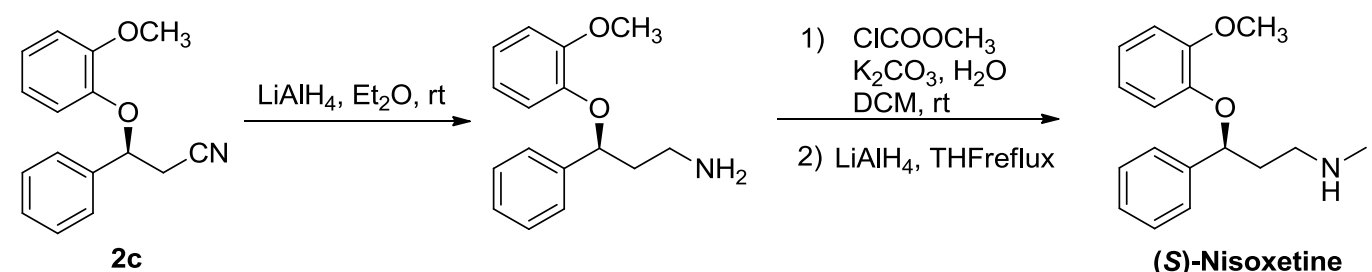

(S)-Nisoxetine

In a flame-dried $25 \mathrm{~mL}$ round bottom flask, $2 \mathrm{c}(0.5 \mathrm{mmol})$ was dissolved in anhydrous diethyl ether $(6 \mathrm{~mL})$ under an atmosphere of $\mathrm{N}_{2} \cdot \mathrm{LiAlH}_{4}(2 \mathrm{mmol})$ was added quickly in one portion at $0{ }^{\circ} \mathrm{C}$. When the exotherm ceased, the reaction mixture was warmed up to room temperature and stirred. After the reaction was completed, the reaction mixture was quenched with several drops of $\mathrm{H}_{2} \mathrm{O}$ at $0{ }^{\circ} \mathrm{C}$, and several drops of $2 \mathrm{M} \mathrm{NaOH}$ were added so that a thick white precipitate formed. This suspension was filtered through a pad of Celite washing with $\mathrm{Et}_{2} \mathrm{O}$. The resulting clear colourless solution was evaporated under reduced pressure to afford intermediate $\mathbf{1}$, which was used without further purification.

To a solution of intermediate $1(0.4 \mathrm{mmol})$ in DCM $(4 \mathrm{~mL})$ was added methyl chloroformate $(0.48 \mathrm{mmol})$. The solution was cooled to $0{ }^{\circ} \mathrm{C}$ and $\mathrm{K}_{2} \mathrm{CO}_{3}(1.6 \mathrm{mmol})$ and $\mathrm{H}_{2} \mathrm{O}(4 \mathrm{~mL})$ were added. The reaction was warmed up to room temperature and stirred. After the reaction was completed, $\mathrm{H}_{2} \mathrm{O}(5 \mathrm{~mL})$ was added and the reaction mixture was extracted with DCM, dried over $\mathrm{Na}_{2} \mathrm{SO}_{4}$ and evaporated under reduced pressure. The residue was purified by silica gel chromatography to afford intermediate 2.

In a flame-dried $25 \mathrm{~mL}$ round bottom flask, intermediate $2(0.2 \mathrm{mmol})$ was dissolved in anhydrous THF (4 mL) under an atmosphere of $\mathrm{N}_{2} \cdot \mathrm{LiAlH}_{4}(0.8 \mathrm{mmol})$ was added quickly in one portion at $0{ }^{\circ} \mathrm{C}$. When the exotherm ceased, the reaction mixture was warmed up to room temperature and stirred at reflux. After the reaction was completed, the reaction mixture was quenched with several drops of $\mathrm{H}_{2} \mathrm{O}$ at $0{ }^{\circ} \mathrm{C}$, and several drops of $2 \mathrm{M} \mathrm{NaOH}$ were added so that a thick white precipitate formed. This suspension was filtered through a pad of Celite washing with $\mathrm{Et}_{2} \mathrm{O}$. The resulting solution was evaporated under reduced pressuren and purified by silica gel chromatography to afford $(S)$-Nisoxetine. 
colorless oil; Yield: $89 \%$; $48.3 \mathrm{mg}(\mathrm{MeOH}: \mathrm{DCM}=1: 1) ;[\alpha]_{\mathrm{D}}{ }^{25}=-32.8(\mathrm{c}=1.0$, $\left.\mathrm{CHCl}_{3}\right)$; HPLC condition: Lux 5u Cellulose-1 $(250 \times 4.60 \mathrm{~mm})$, ipa:hex = 5:95, 1.0 $\mathrm{mL} / \mathrm{min}, 254 \mathrm{~nm} ; \mathrm{t}_{\mathrm{A}}=25.7 \min$ (major), $\mathrm{t}_{\mathrm{B}}=28.3 \min$ (minor); ${ }^{1} \mathrm{H} \mathrm{NMR}(400 \mathrm{MHz}$, $\left.\mathrm{CDCl}_{3}\right) \delta: 7.38-7.23(\mathrm{~m}, 5 \mathrm{H}), 6.88-6.83(\mathrm{~m}, 2 \mathrm{H}), 6.72-6.66(\mathrm{~m}, 2 \mathrm{H}), 5.20(\mathrm{dd}, J=4.0$ $\mathrm{Hz}, 8.4 \mathrm{~Hz}), 3.88(\mathrm{~s}, 3 \mathrm{H}), 2.84-2.76(\mathrm{~m}, 2 \mathrm{H}), 2.44(\mathrm{~s}, 3 \mathrm{H}), 2.30-2.21(\mathrm{~m}, 1 \mathrm{H})$, 2.09-2.03 (m, 1H). ${ }^{13} \mathrm{C} \mathrm{NMR}\left(100 \mathrm{MHz}, \mathrm{CDCl}_{3}\right) \delta: 150.6,148.2,142.5,129.1,128.1$, $126.6,122.1,121.3,116.9,112.6,81.2,56.5,49.3,38.9,36.9 .^{[11]}$ 


\section{References}

[1] Zhou, W.; Zhang, Y.; Li, P.; Wang, L. Org. Biomol. Chem. 2012, 10, 7184.

[2] Trofimov, B. A.; Sobenina, L. N.; Mikhaleva, A. b. I.; Ushakov, I. A.; akul'skaya, T. I.; Stepanova, V. Z. V.; Toryashinova, D. y.-S. D.; Mal'kina, A. G.; Elokhina, V. N. Synthesis 2003, 1272.

[3] Jang, K. P.; Hutson, G. E.; Johnston, R. C.; McCusker, E. O.; Cheong, P. H. Y.; Scheidt, K. A. J. Am. Chem. Soc. 2014, 136, 76.

[4] Kong, D.; Li, M.; Wang, R.; Zi, G.; Hou, G. Org. Biomol. Chem. 2016, 14, 1216.

[5] Li, Y.; Wang, Z.; Ding, K. Chem. -Eur. J. 2015, 21, 16387.

[6] Stewart, G. W.; Shevlin, M.; Yamagata, A. D. G.; Gibson, A. W.; Keen, S. P.; Scott, J. P. Org. Lett. 2012, 14, 5440.

[7] Devine, P. N.; Heid Jr, R. M.; Tschaen, D. M. Tetrahedron 1997, 53, 6739.

[8] Ma, D.-Y.; Wang, D.-X.; Pan, J.; Huang, Z.-T.; Wang, M.-X. Tetrahedron: Asymmetry 2008, 19, 322.

[9] Richardson, R. D.; Hernandez-Juan, F. A. Dixon, D. J. Synlett 2006, 77.

[10] Lifchits, O.; Alberico, D.; Zakharian, I.; Charette, A. B. J. Org. Chem. 2008, 73, 6838.

[11] Rej, R. K.; Das, T.; Hazra, S.; Nanda, S. Tetrahedron: Asymmetry 2013, 24, 913. 
7. NMR, GC and HPLC spectra of compounds

(Z)-3-phenoxy-3-phenylacrylonitrile (1a)
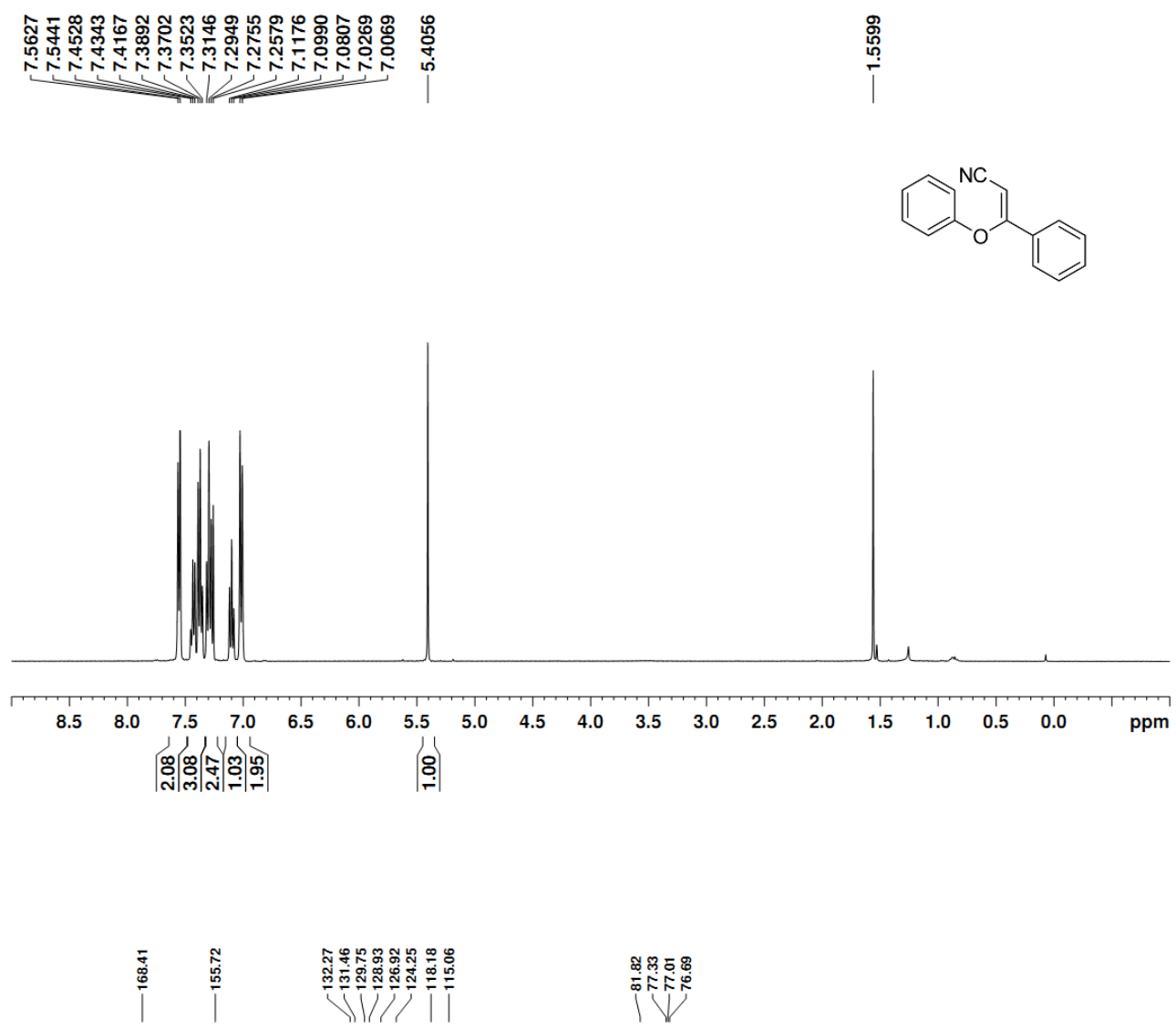

జ

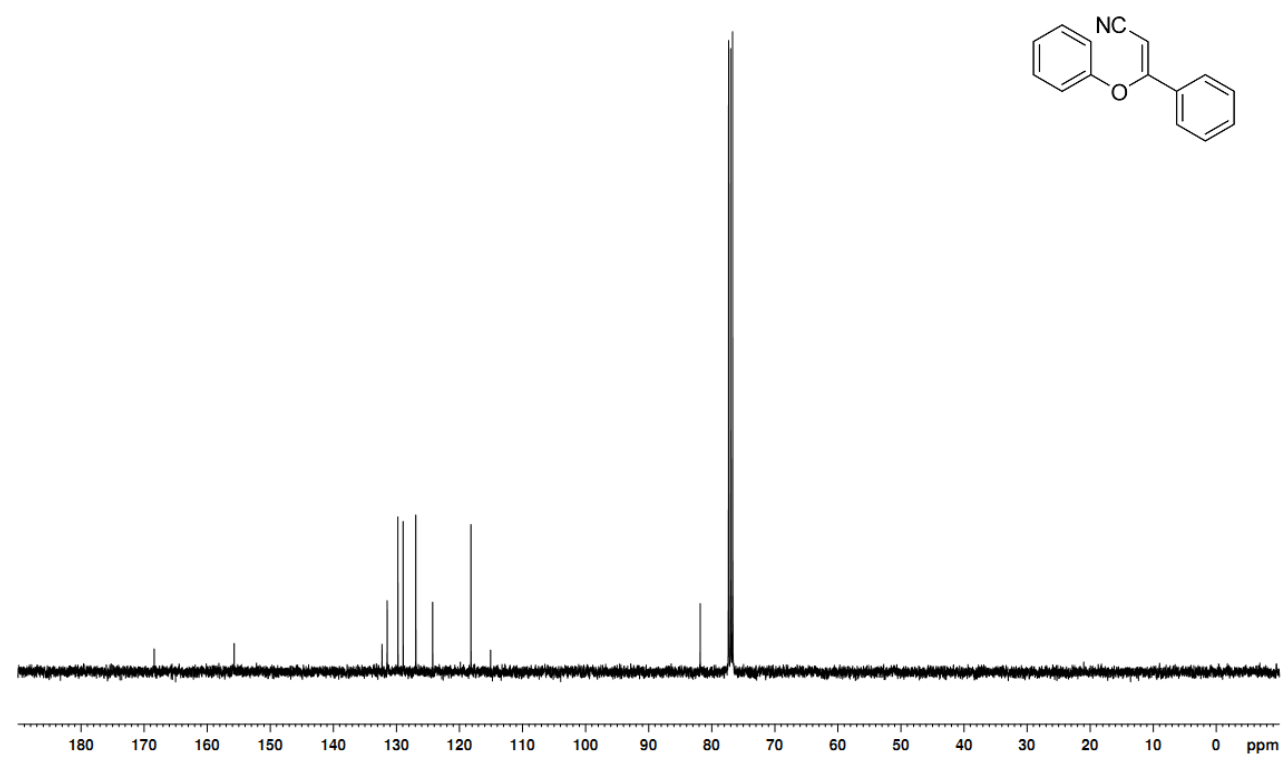


(Z)-3-phenyl-3-(o-tolyloxy)acrylonitrile (1b)

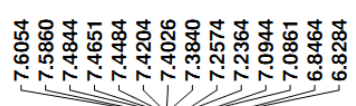

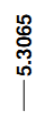

i
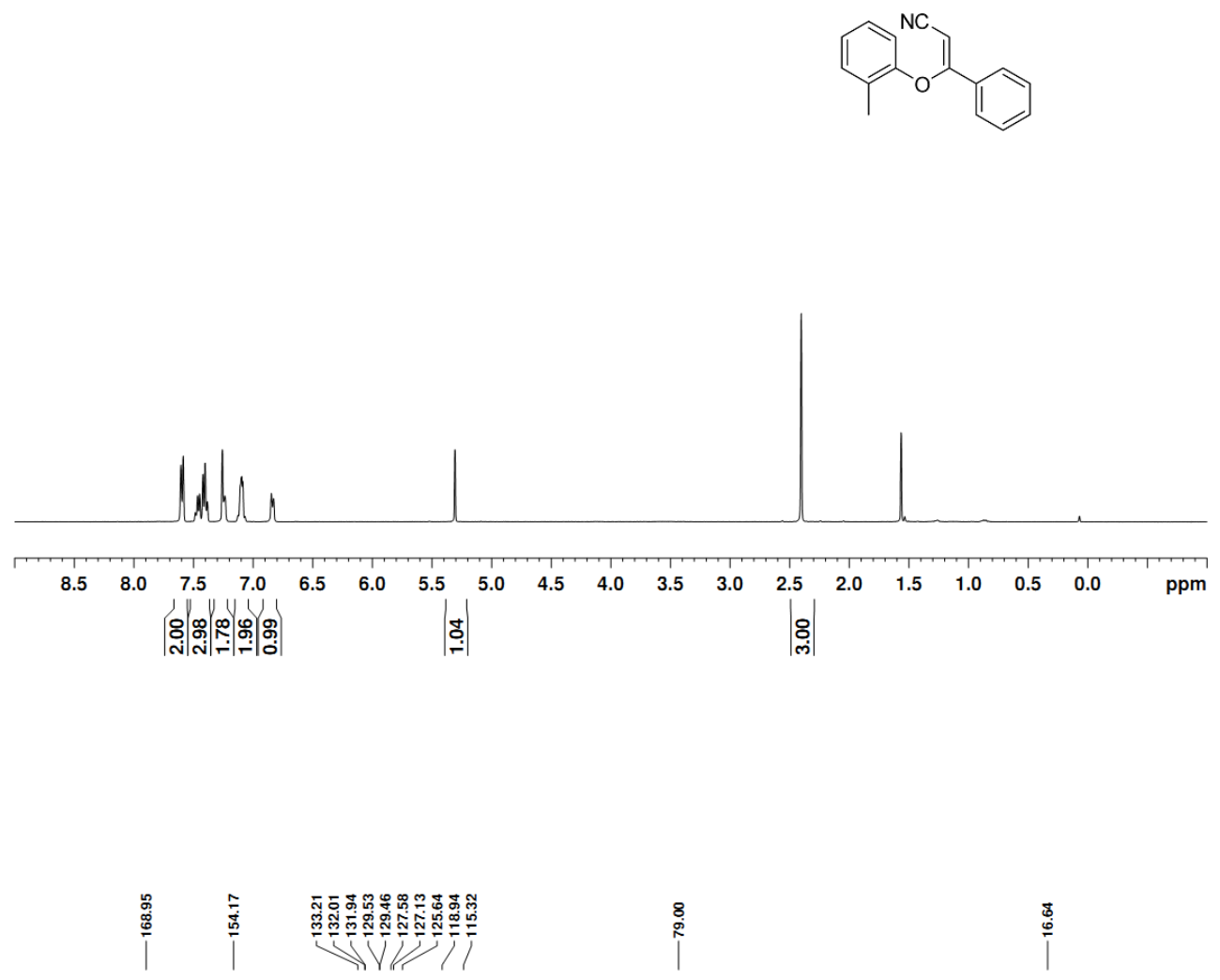

i

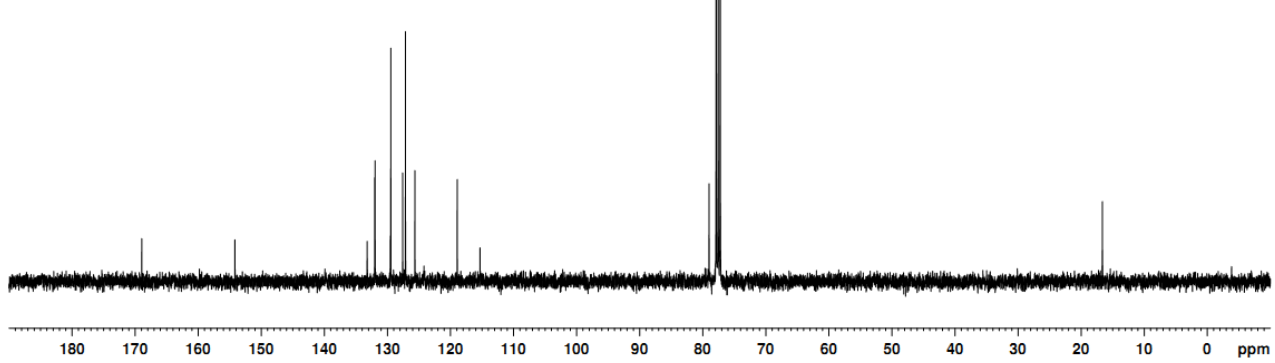

S22 
(Z)-3-(2-methoxyphenoxy)-3-phenylacrylonitrile (1c)
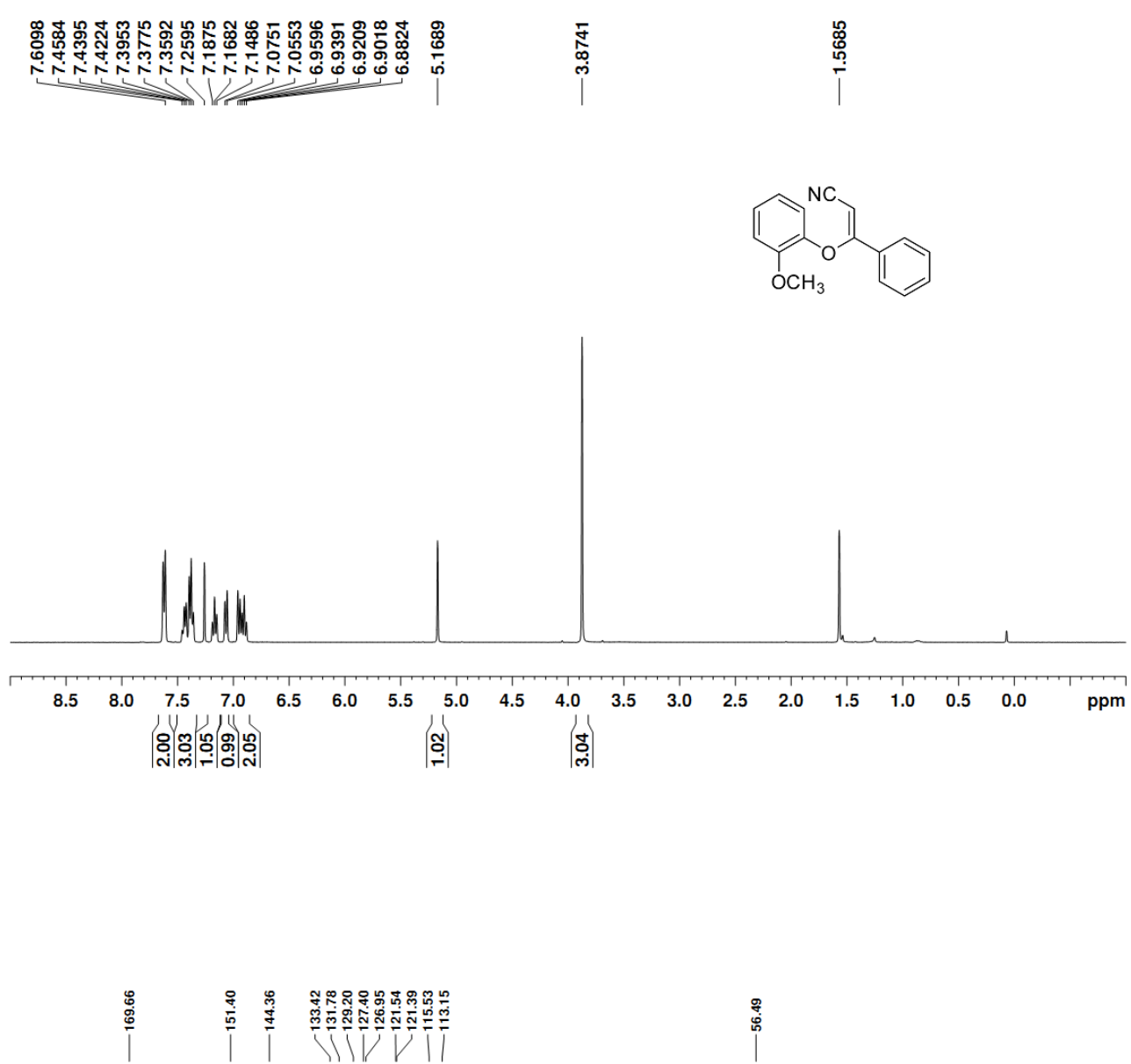

|

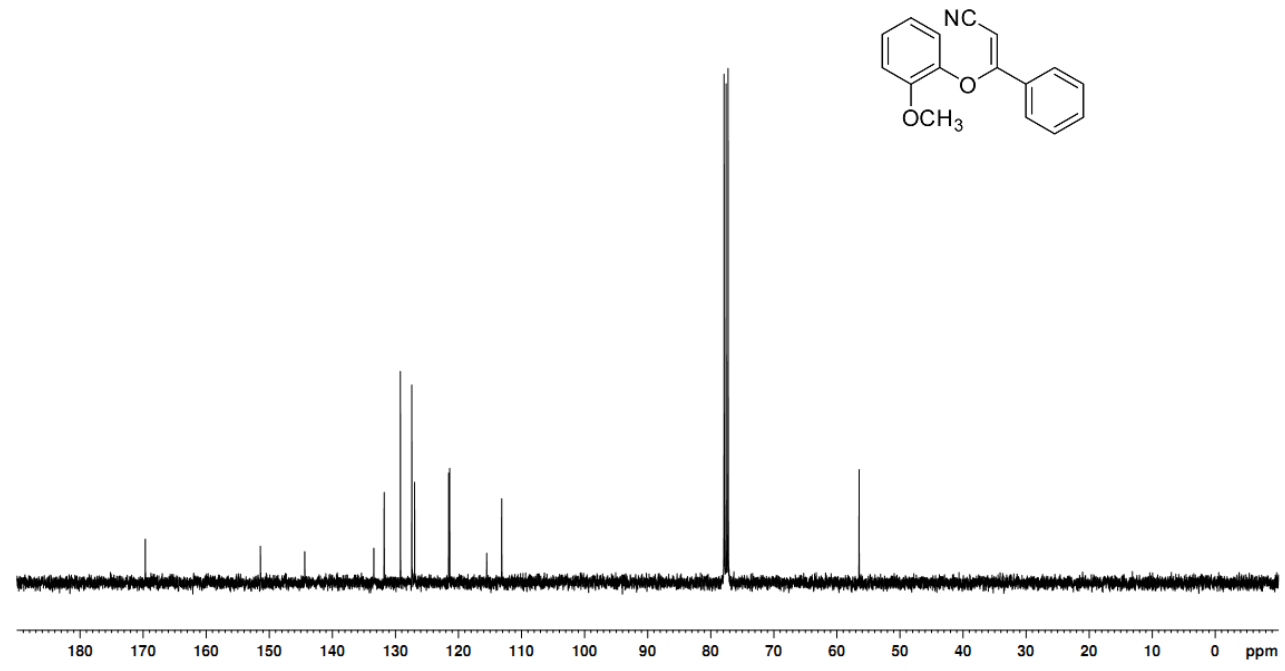


(Z)-3-(2-chlorophenoxy)-3-phenylacrylonitrile (1d)
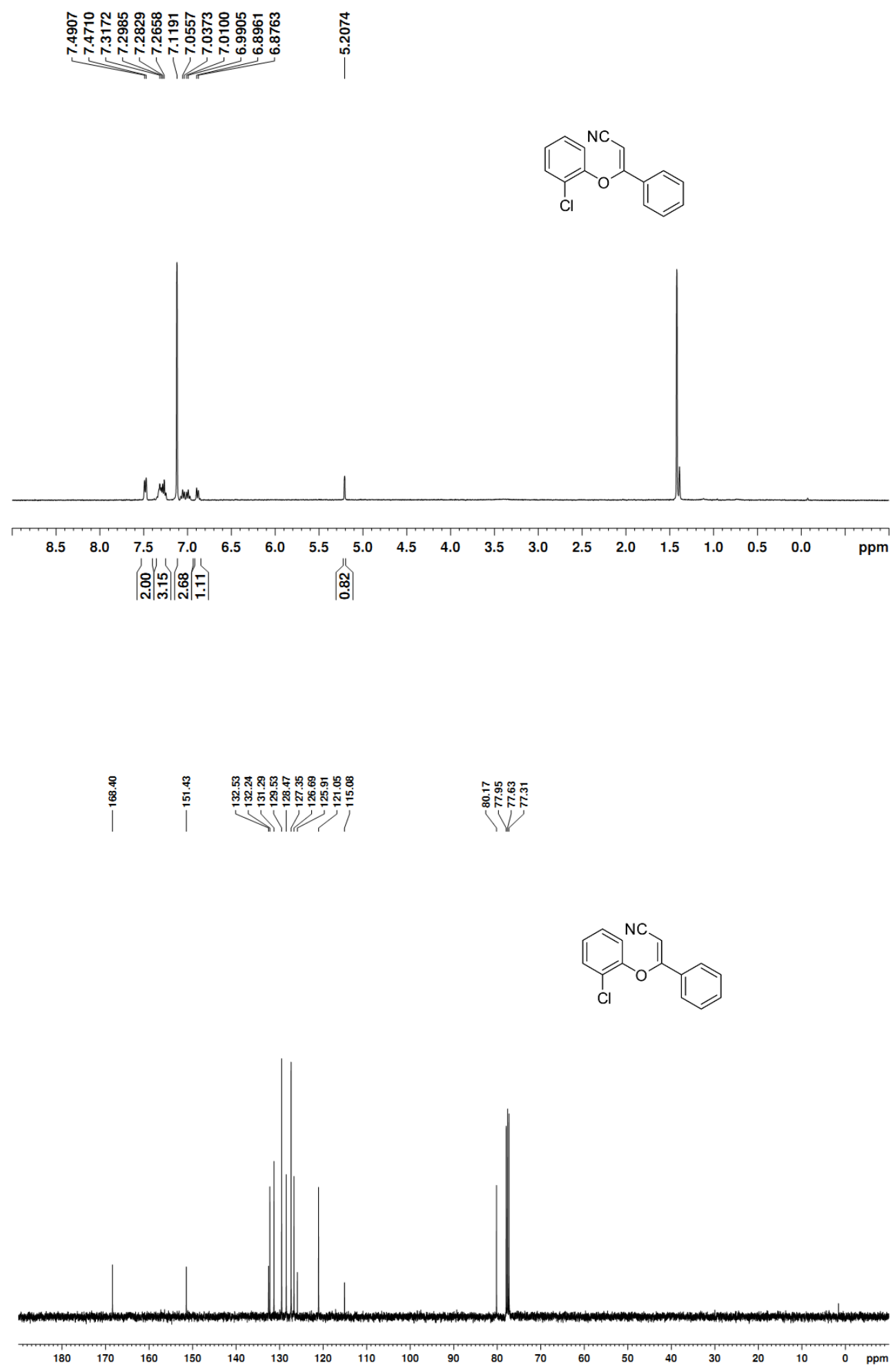
(Z)-3-(3-chlorophenoxy)-3-phenylacrylonitrile (1e)

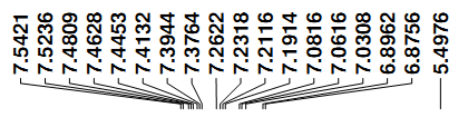
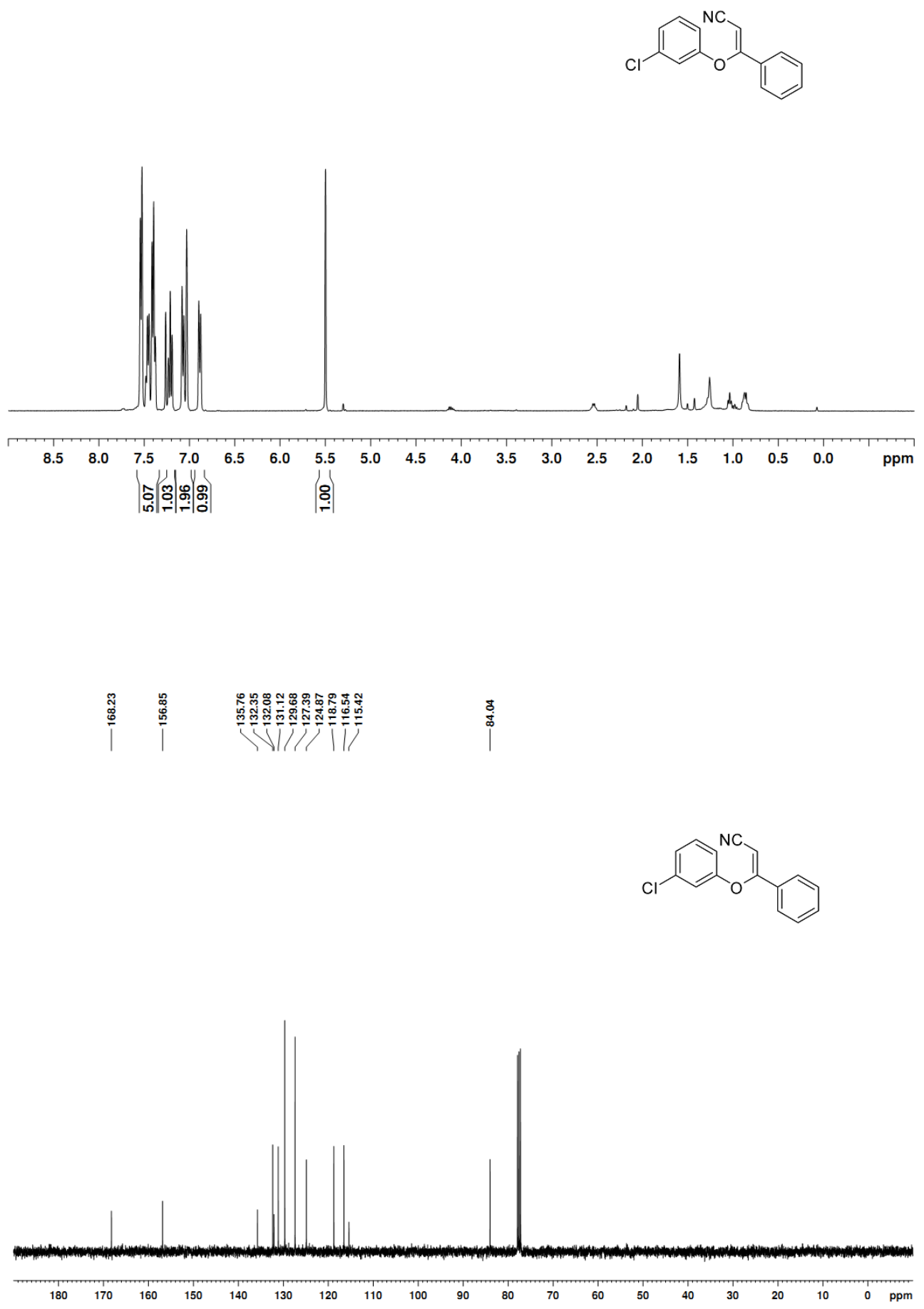

S25 
(Z)-3-phenyl-3-(p-tolyloxy)acrylonitrile (1f)

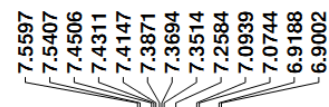

|

ָึ
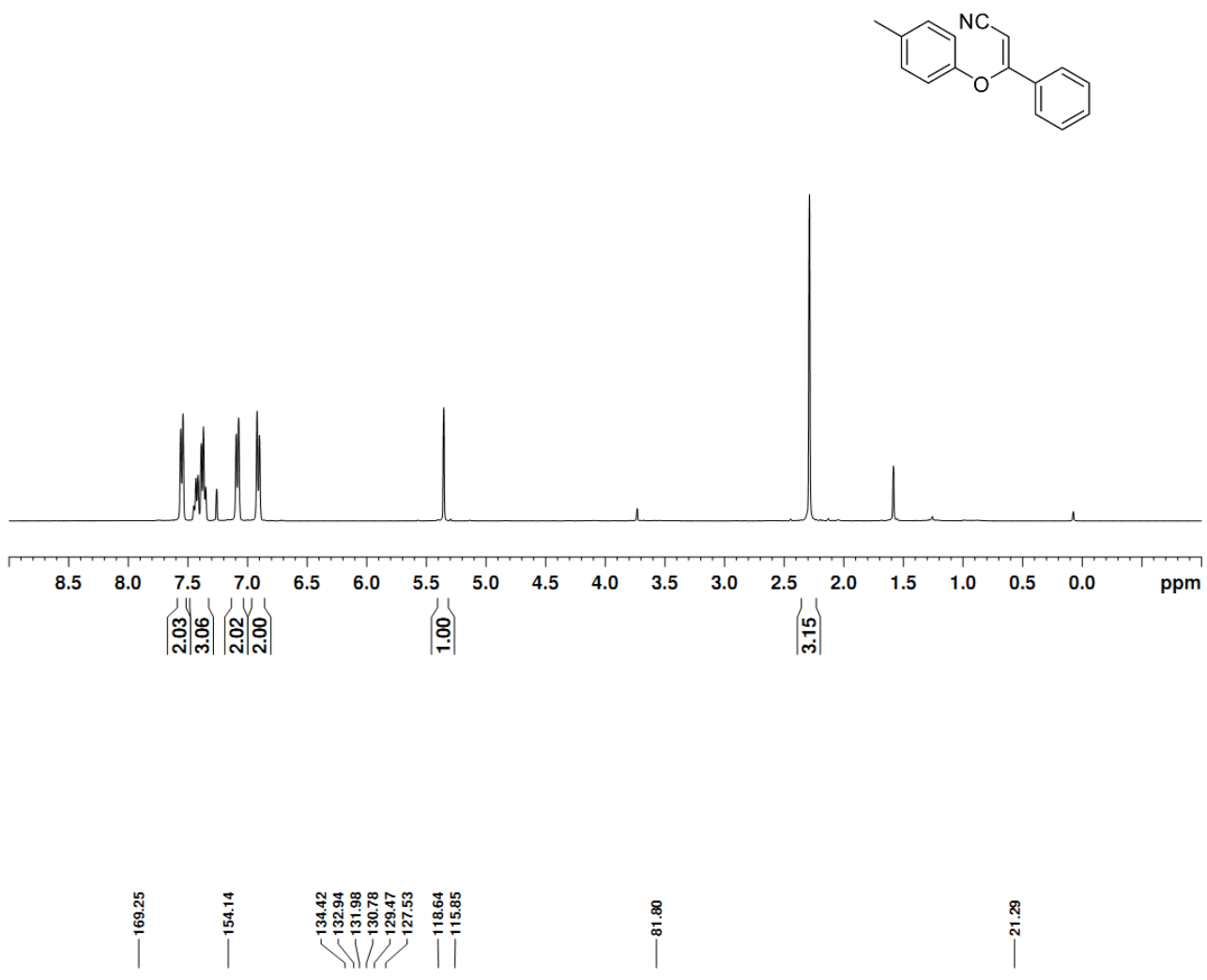

$\stackrel{8}{i}$
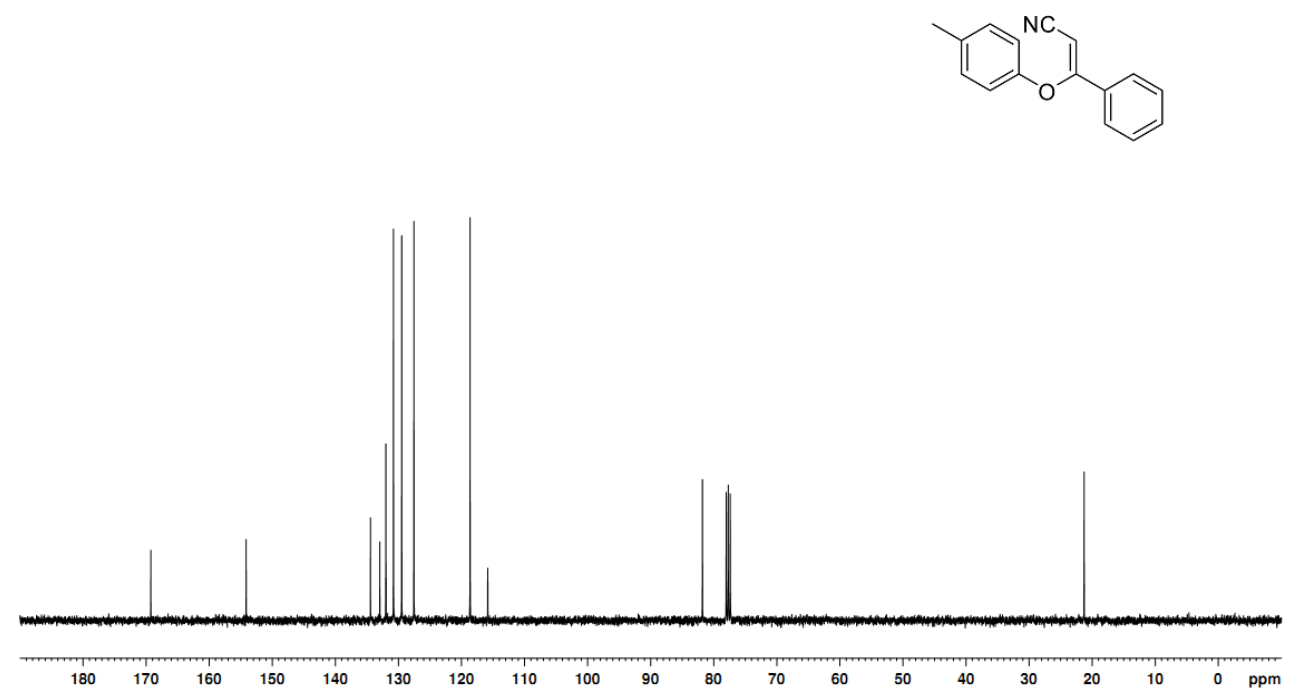

S26 
(Z)-3-(4-chlorophenoxy)-3-phenylacrylonitrile (1g)

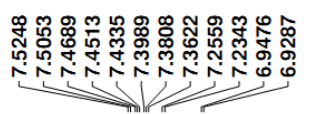

产
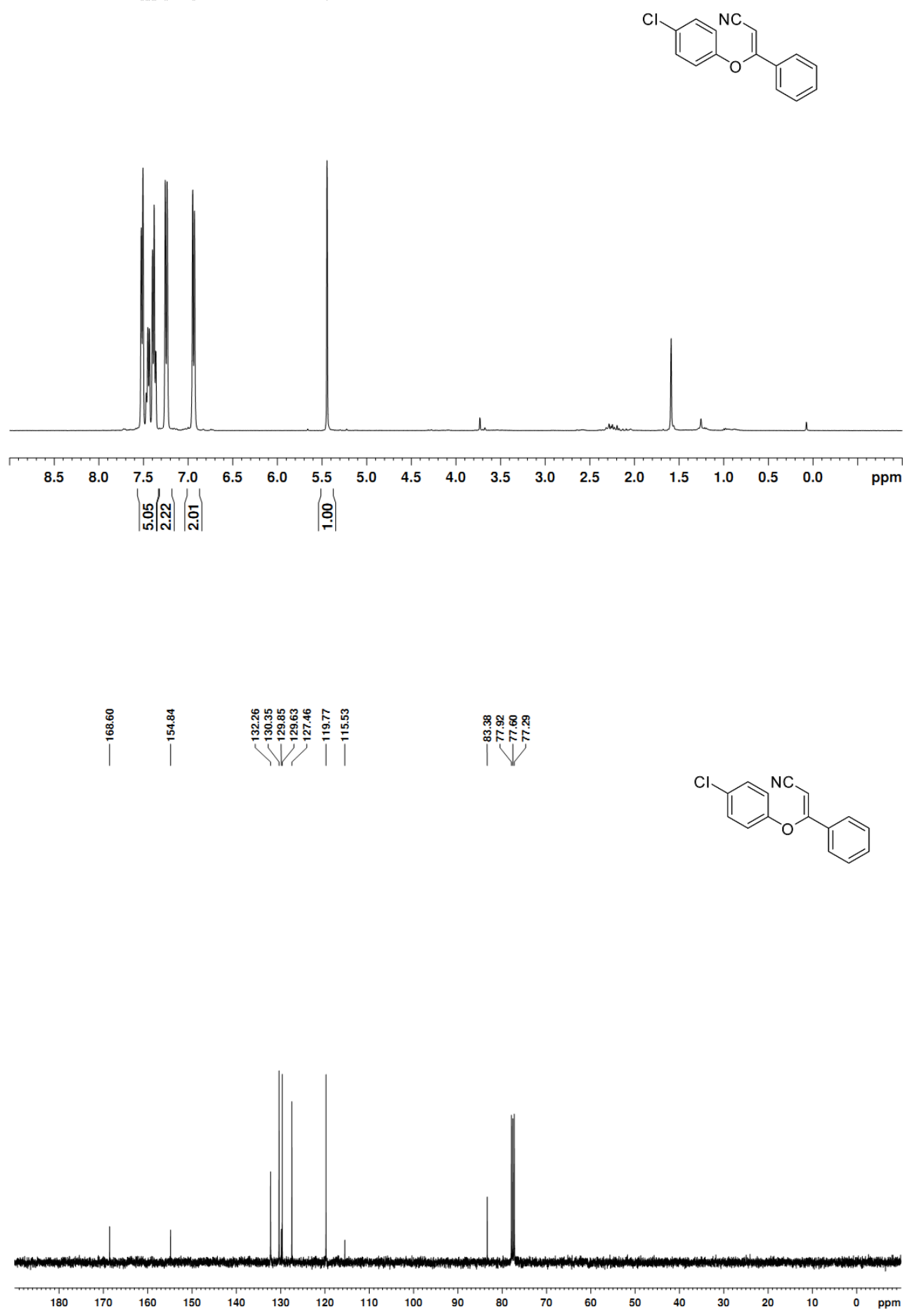

S27 
(Z)-3-phenyl-3-(4-(trifluoromethyl)phenoxy)acrylonitrile (1h)
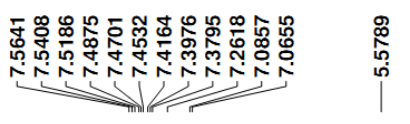
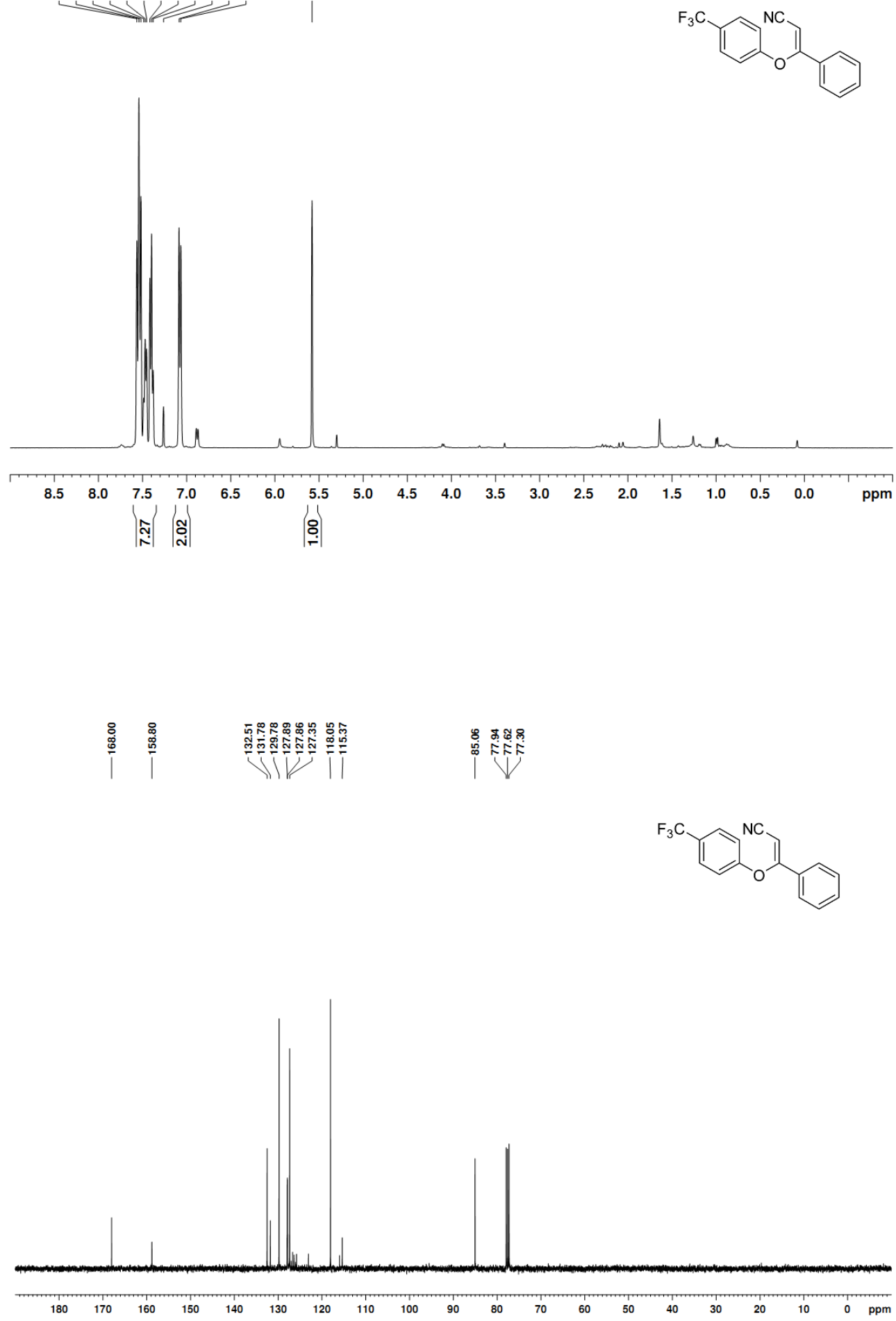

S28 
(Z)-3-(naphthalen-1-yloxy)-3-phenylacrylonitrile (1i)

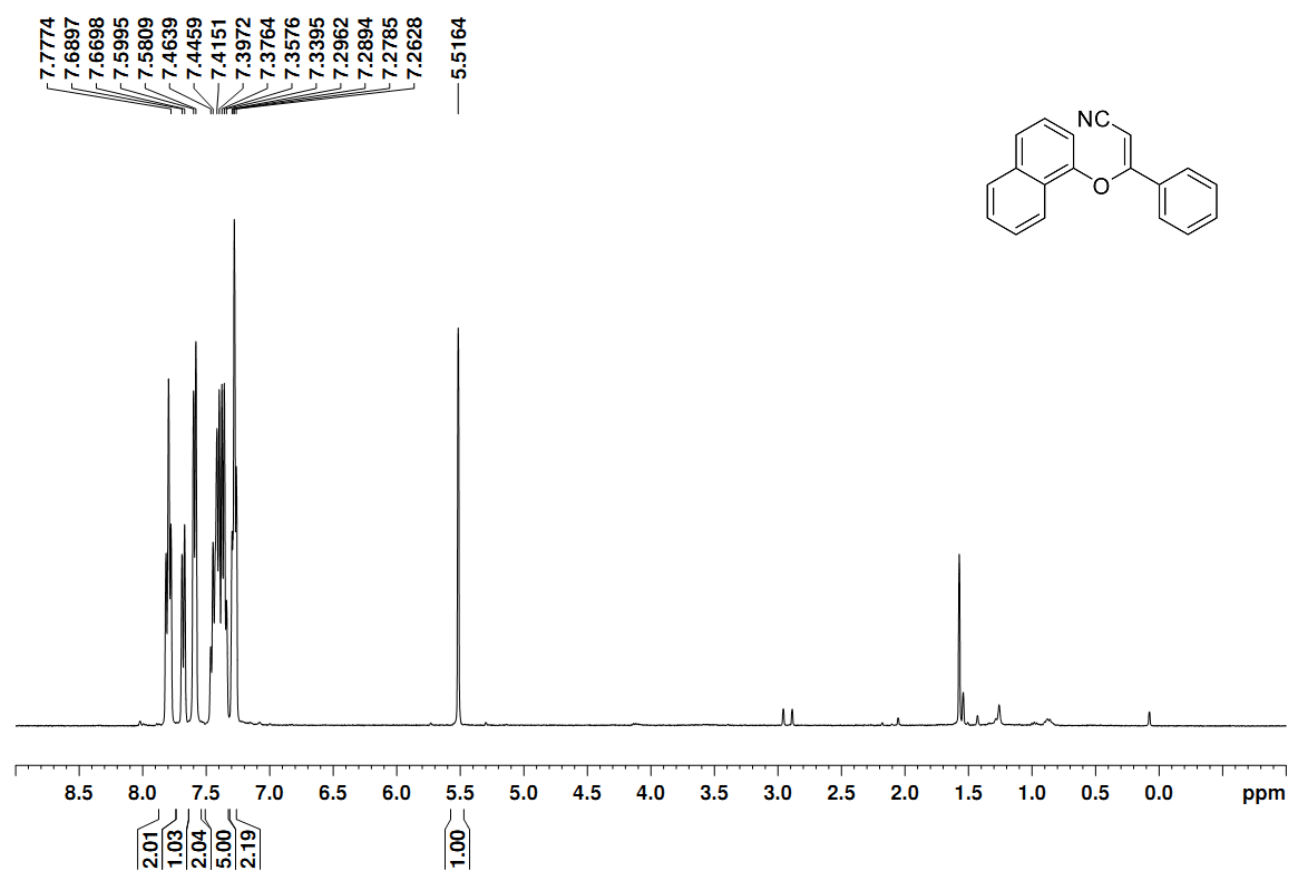

|
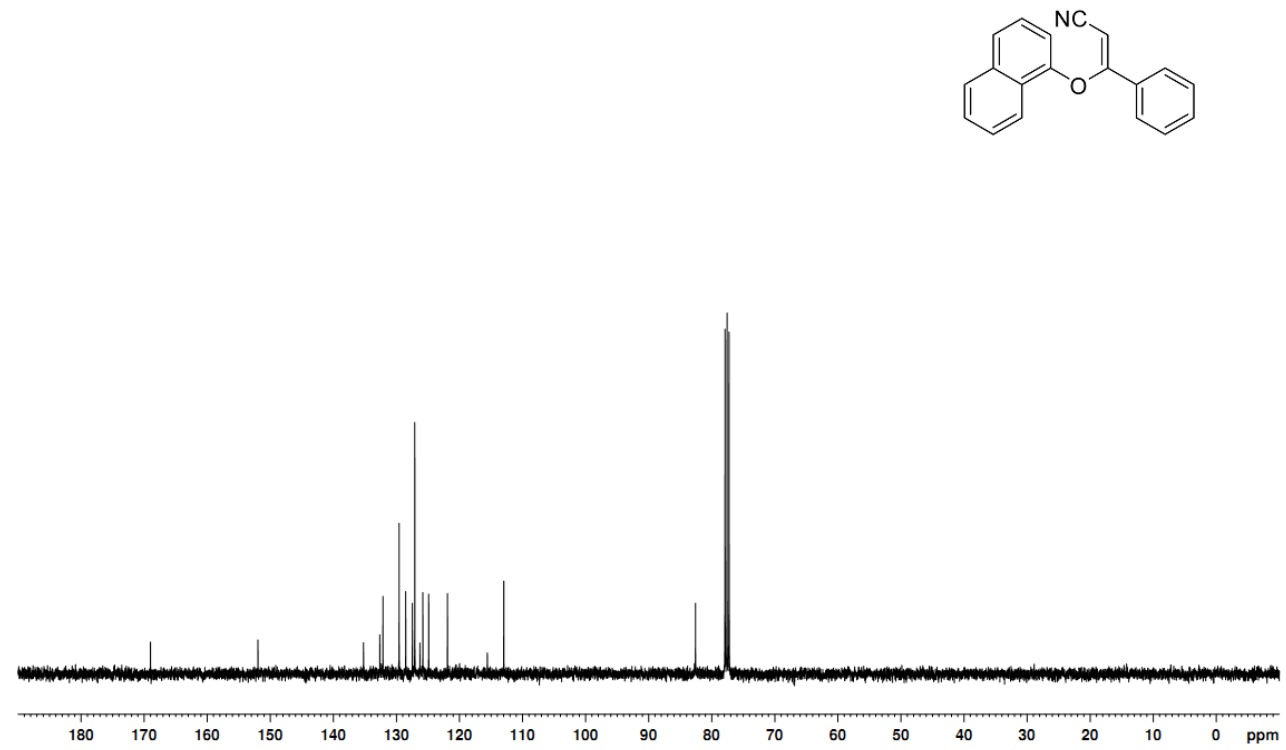
(Z)-3-(naphthalen-2-yloxy)-3-phenylacrylonitrile (1j)

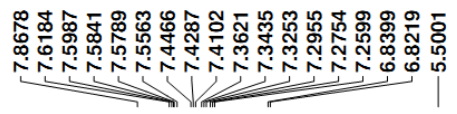
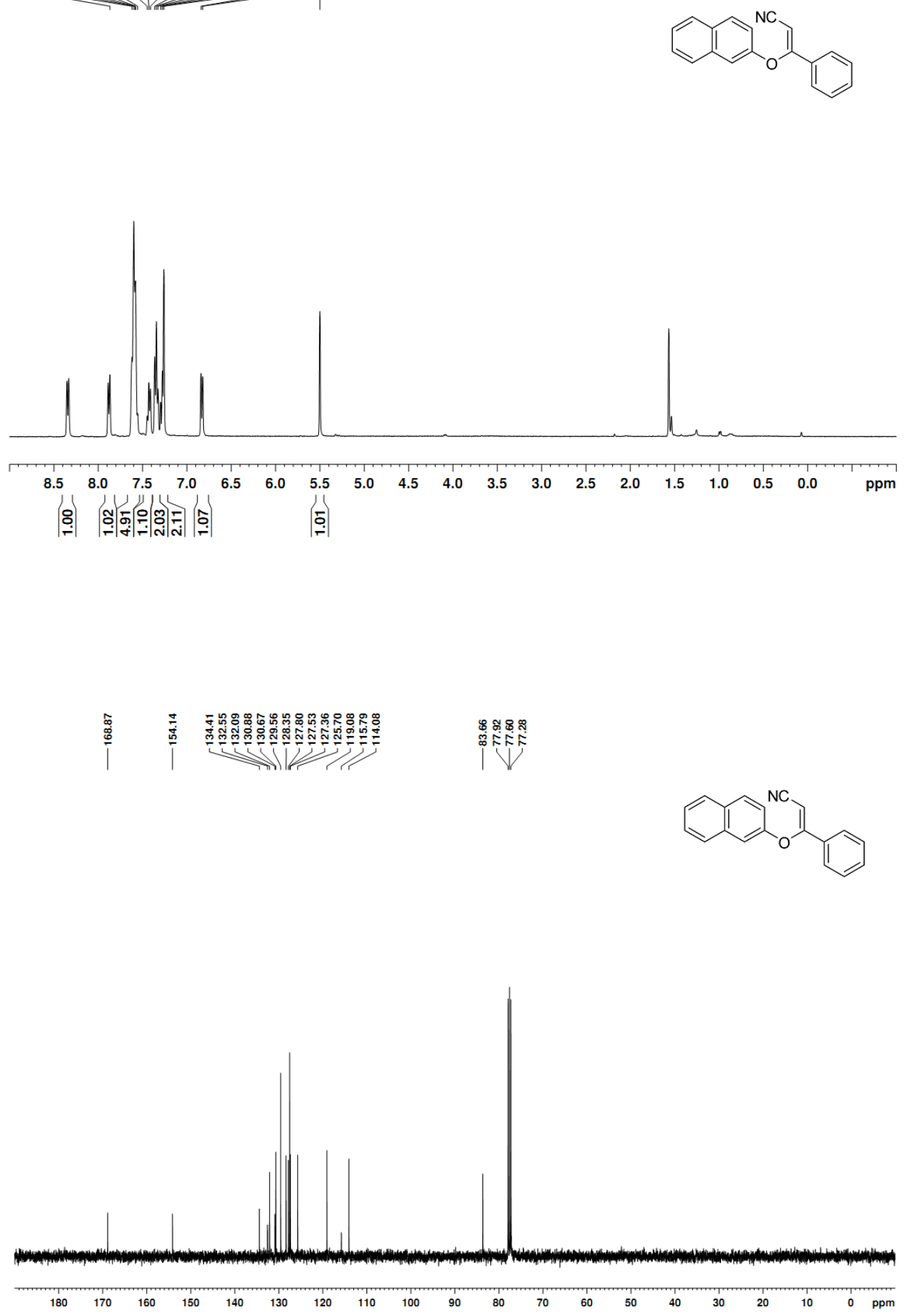

S30 
(E)-3-phenoxy-3-phenylacrylonitrile (1k)

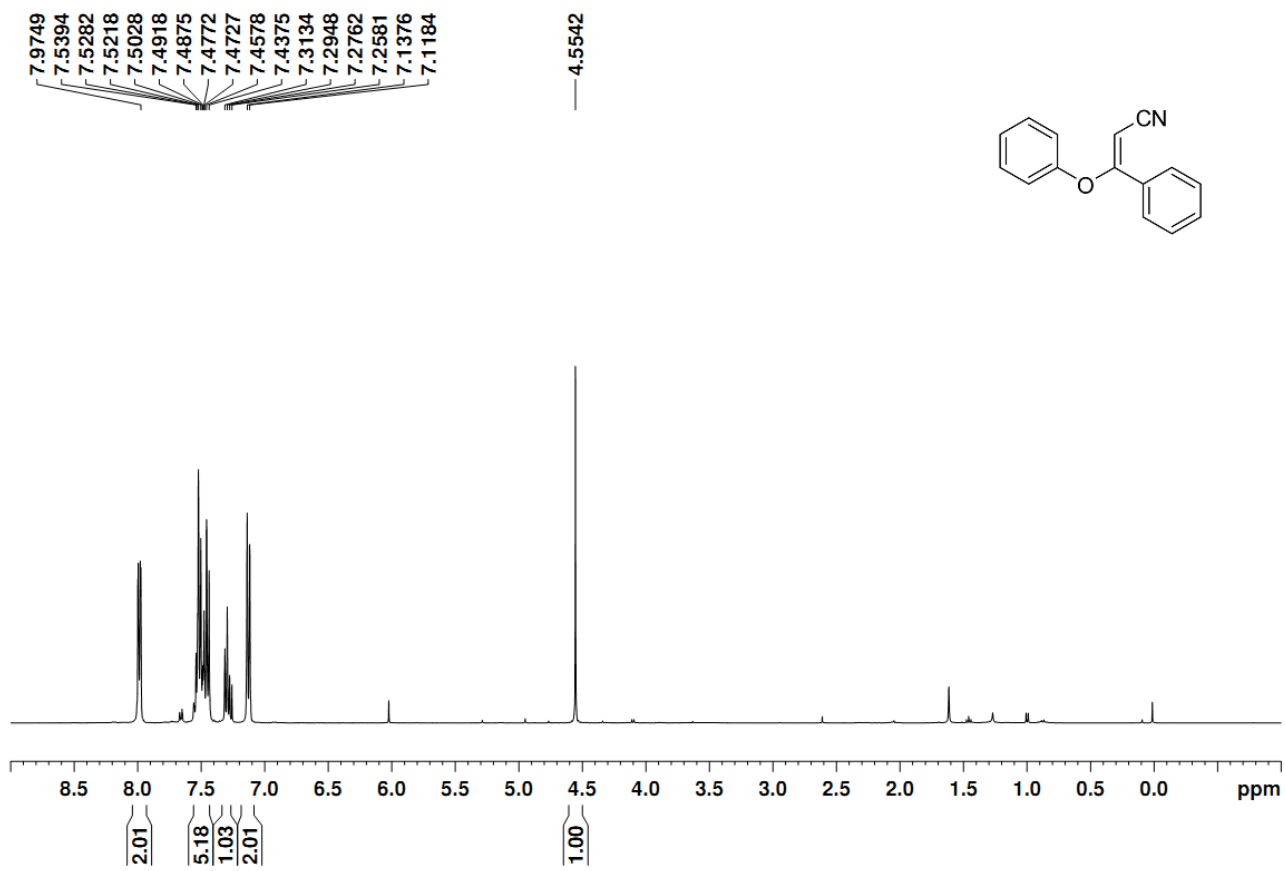

|
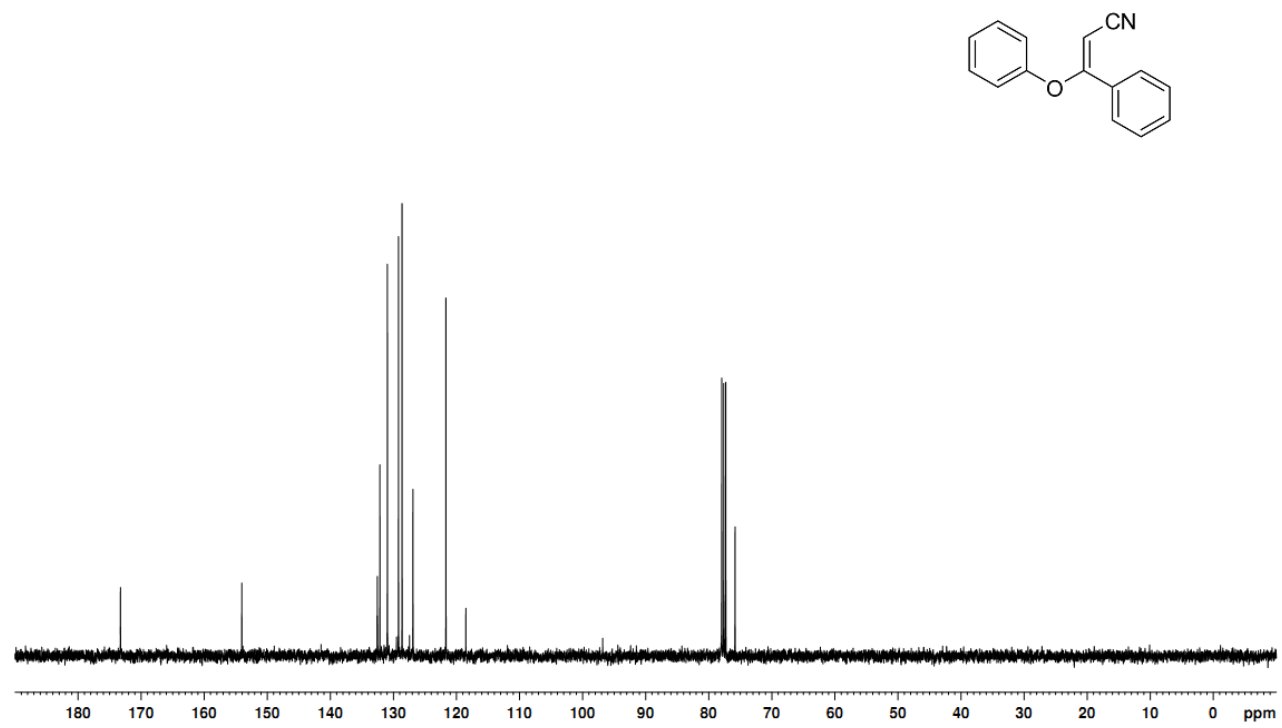
(E)-3-(2-chlorophenoxy)-3-phenylacrylonitrile (11)
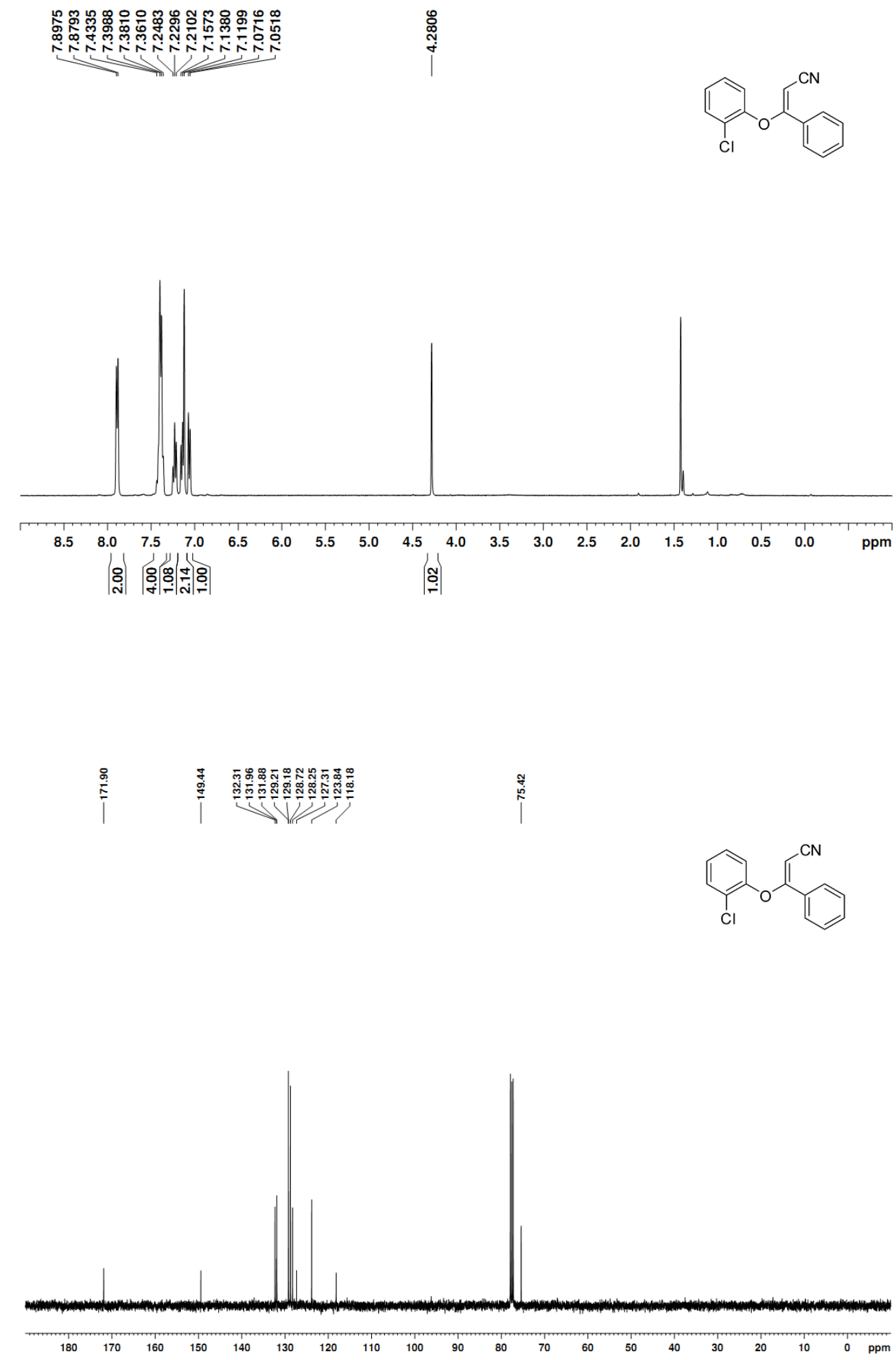
(E)-3-phenyl-3-(p-tolyloxy)acrylonitrile (1m)

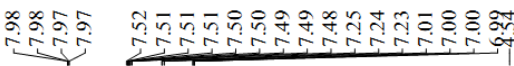

กิ
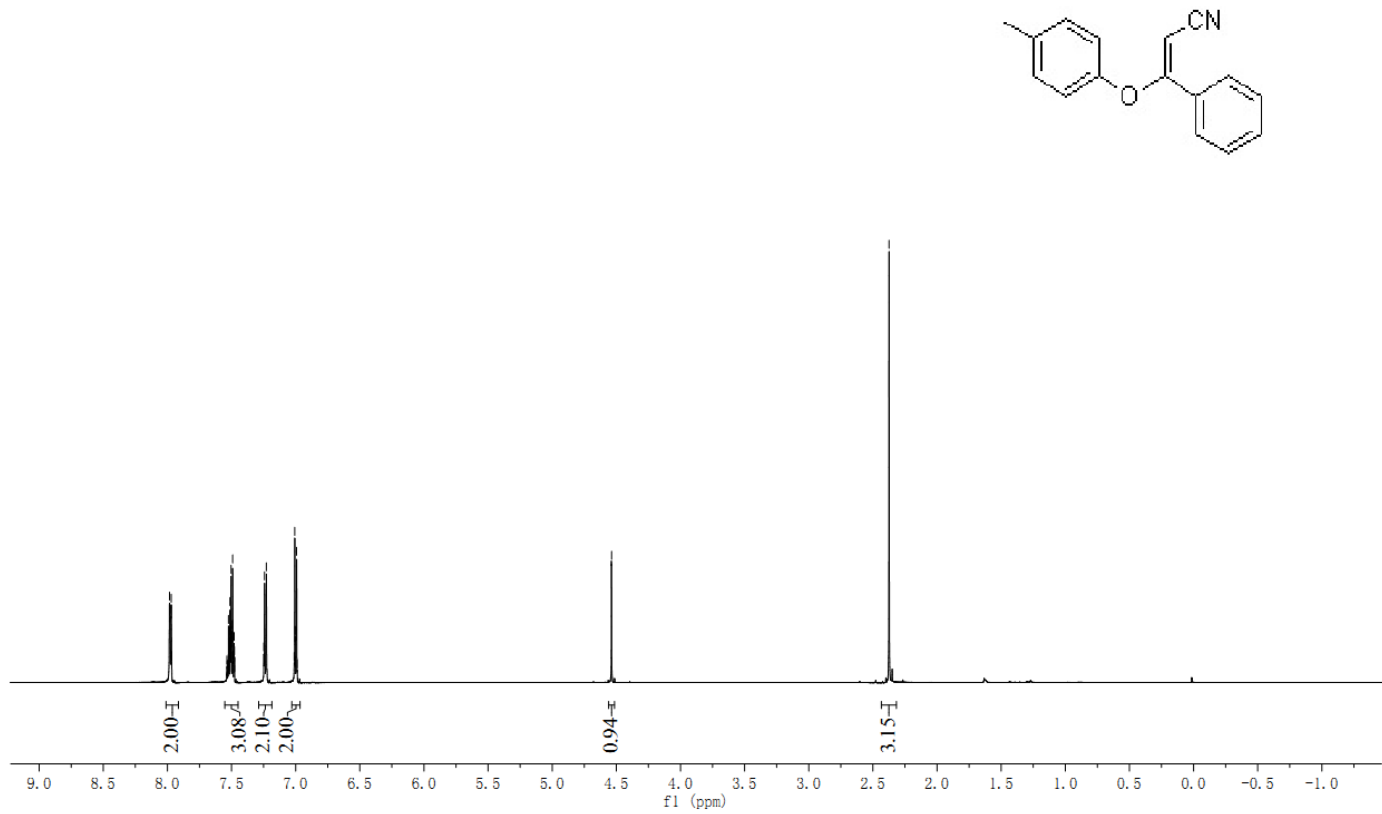

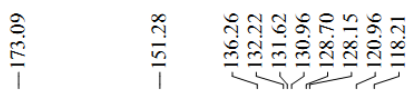

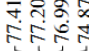

ิิ

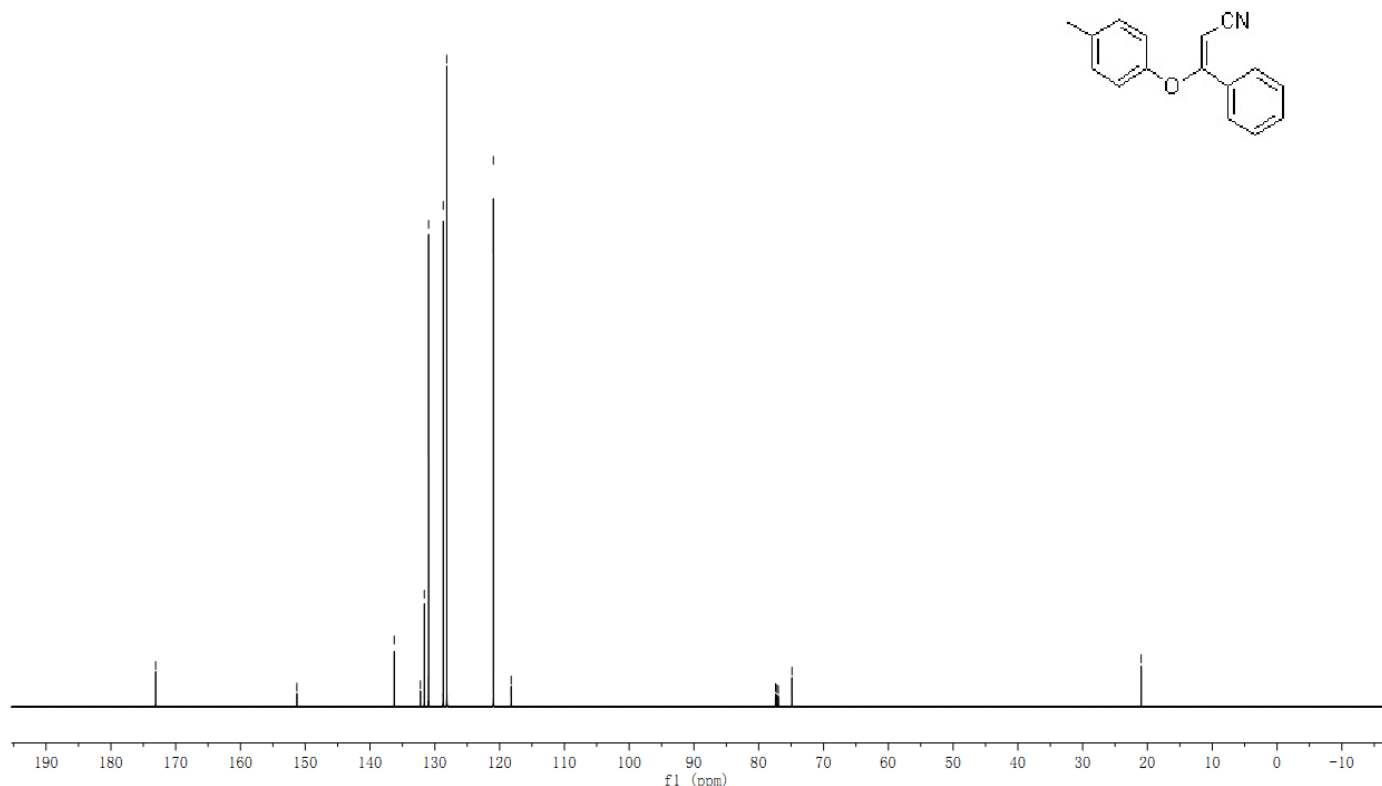


(Z)-3-methoxy-3-phenylacrylonitrile (1n)

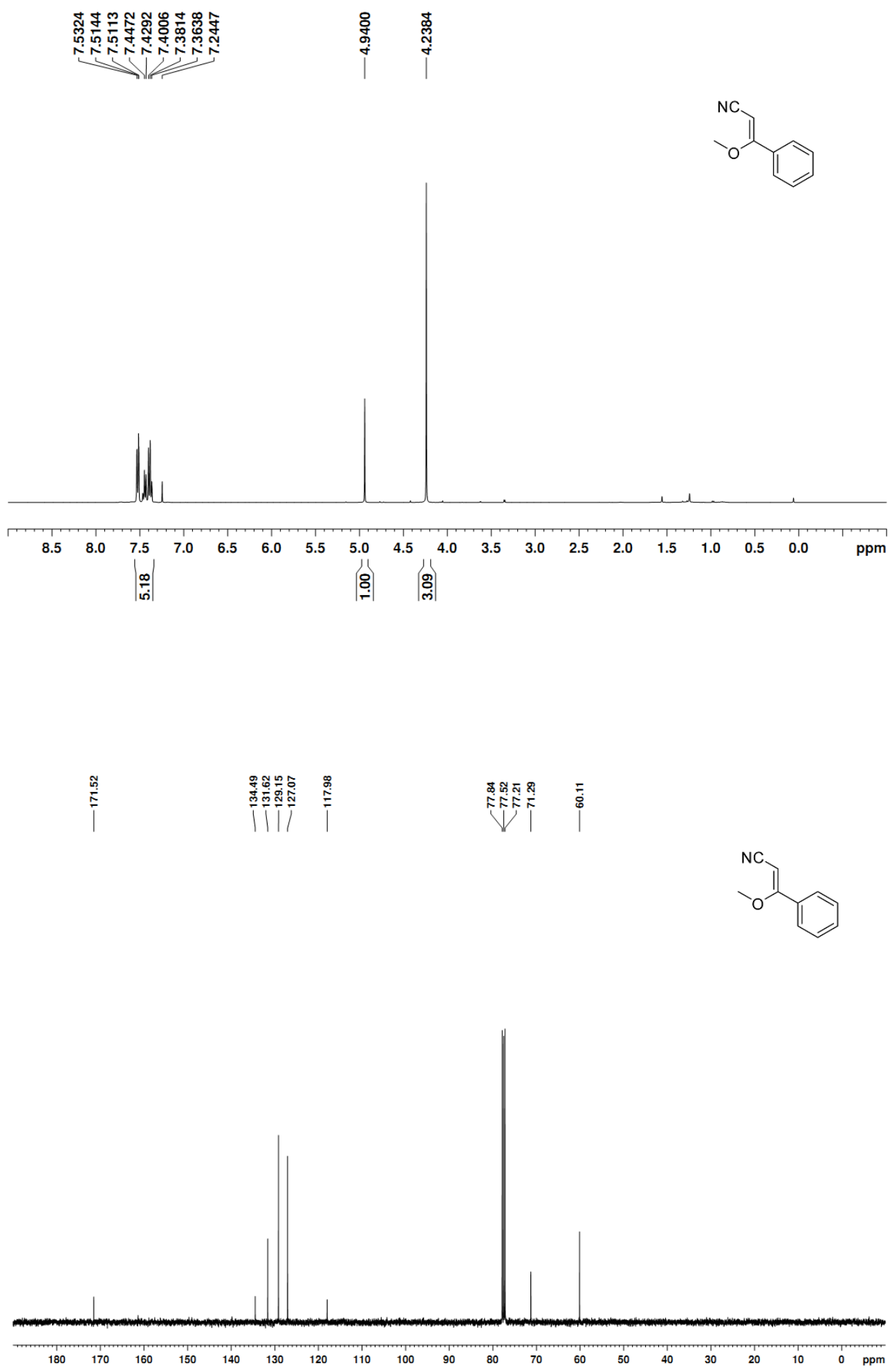


(Z)-3-isopropoxy-3-phenylacrylonitrile (10)

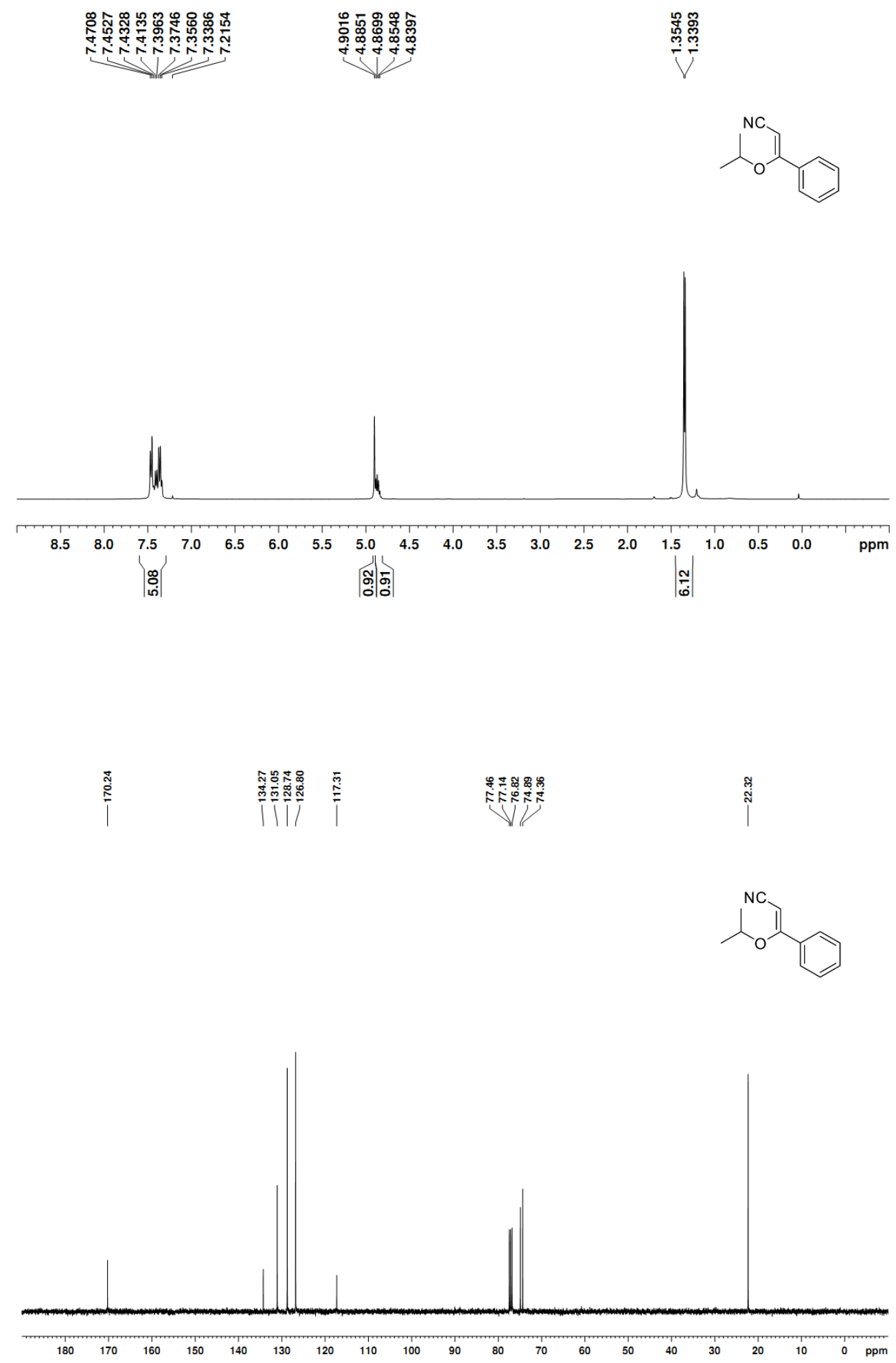


(Z)-3-phenyl-3-(2,2,2-trifluoroethoxy)acrylonitrile (1p)

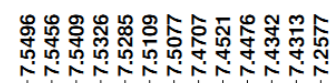

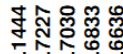

மि
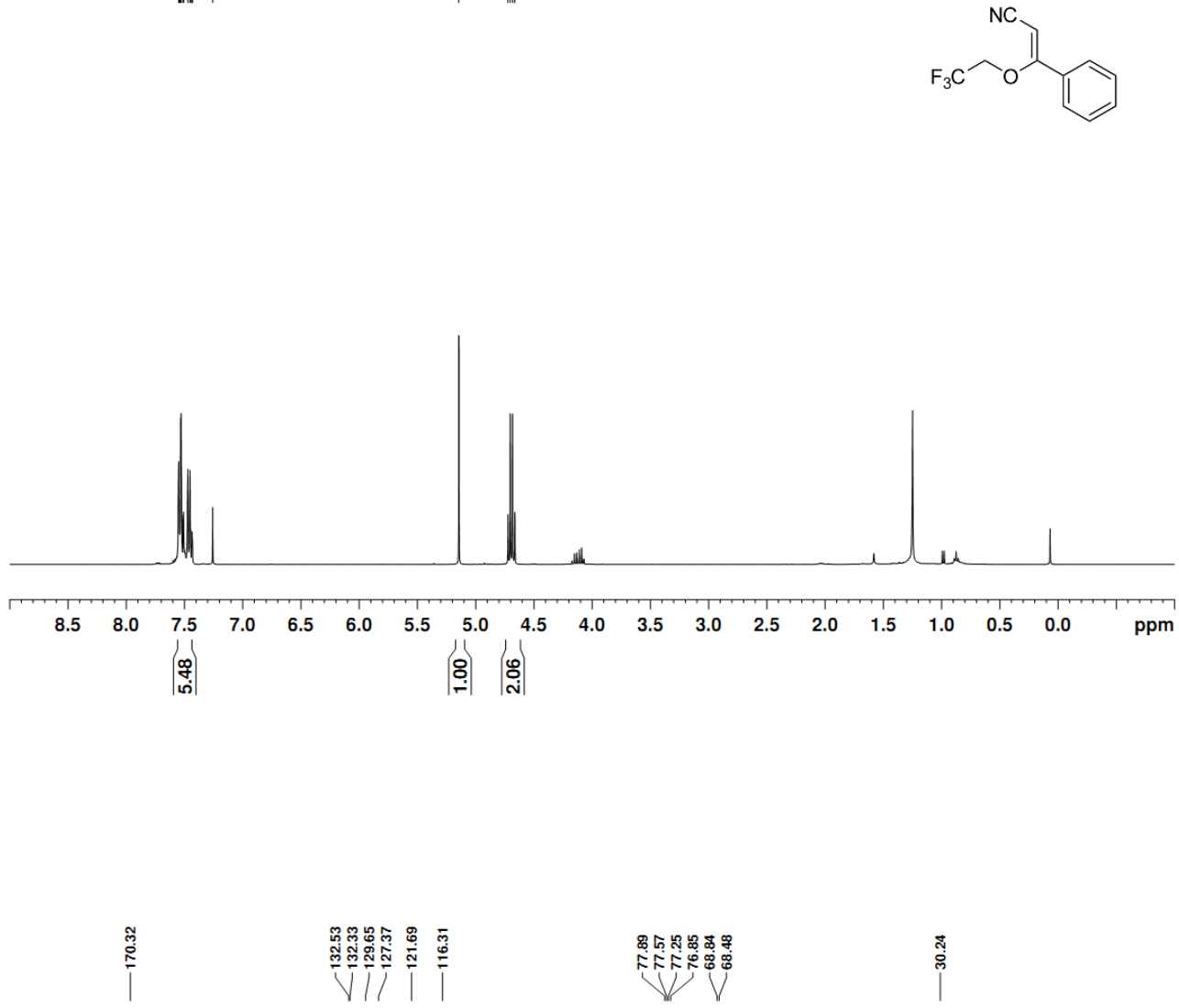

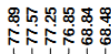

స్ํำ

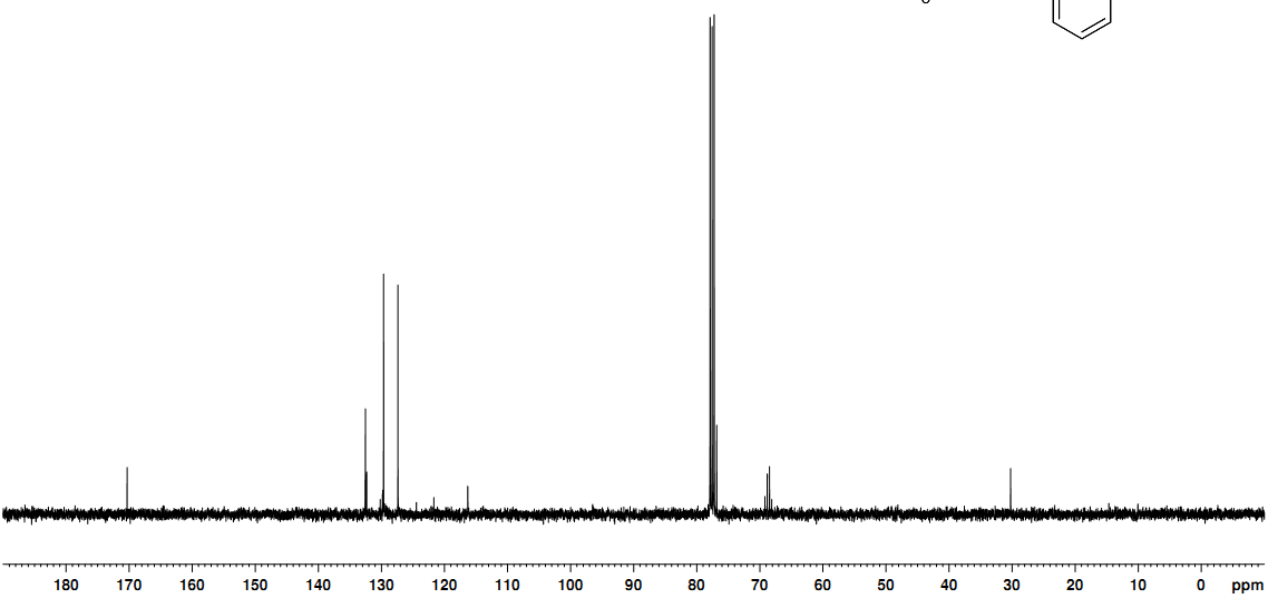



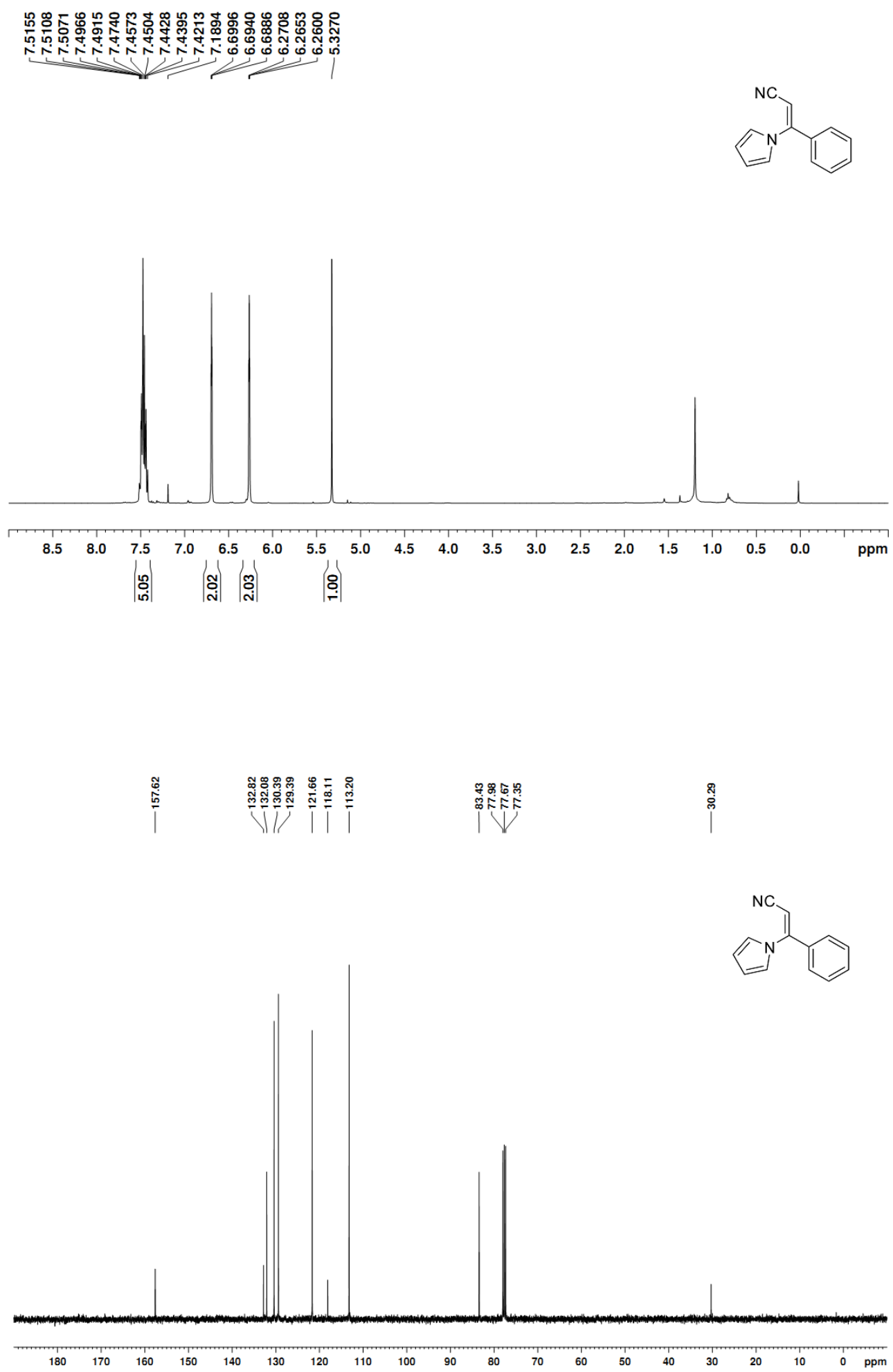
(Z)-dimethyl (2-cyano-1-(p-tolyl)vinyl)phosphonate (1r)

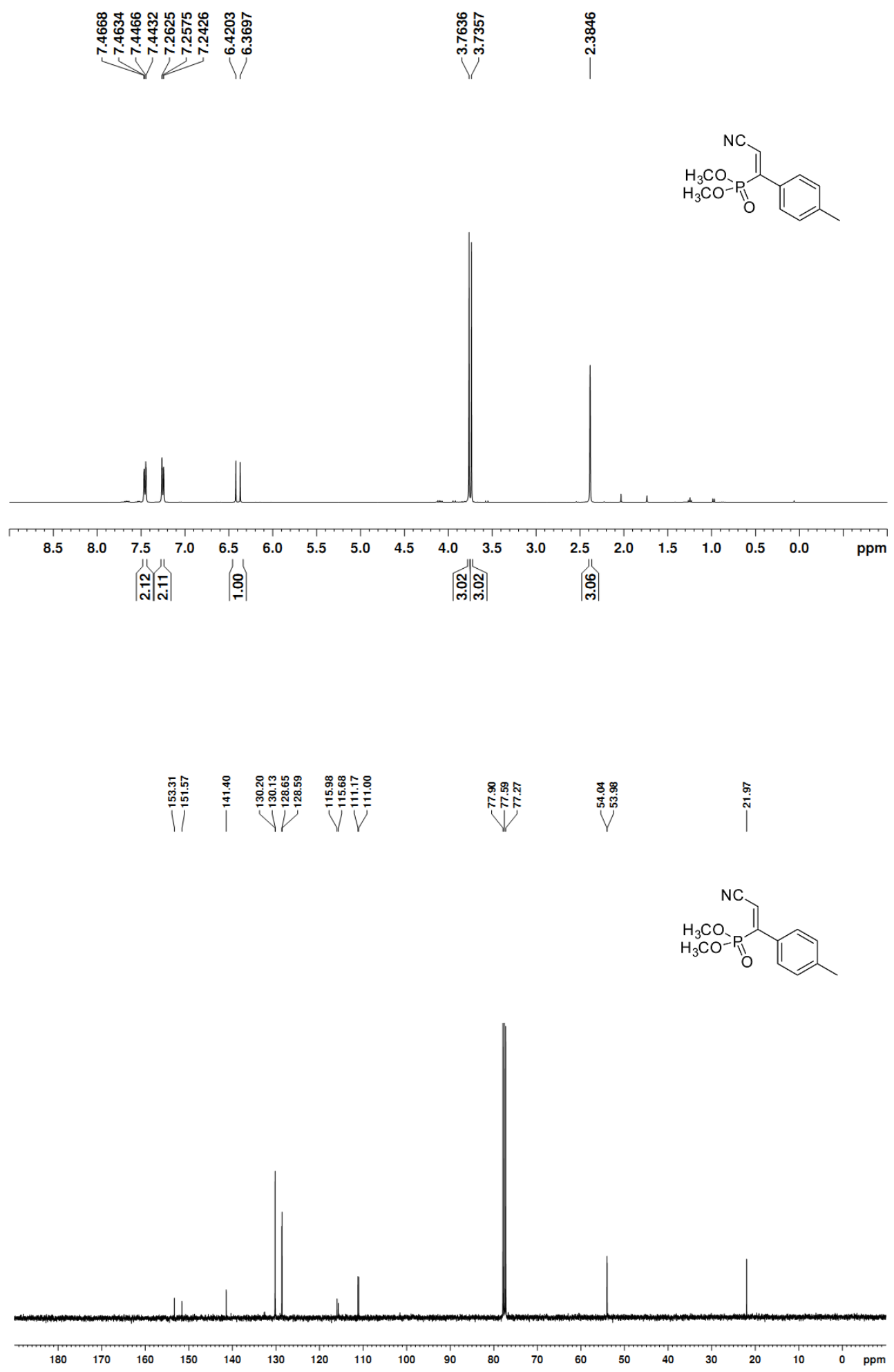


3-phenoxy-3-phenylpropanenitrile (2a and $2 \mathrm{k}$ )

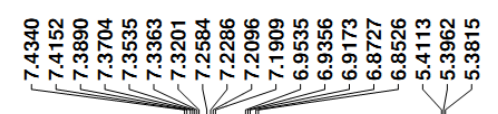

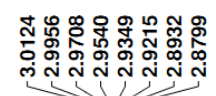
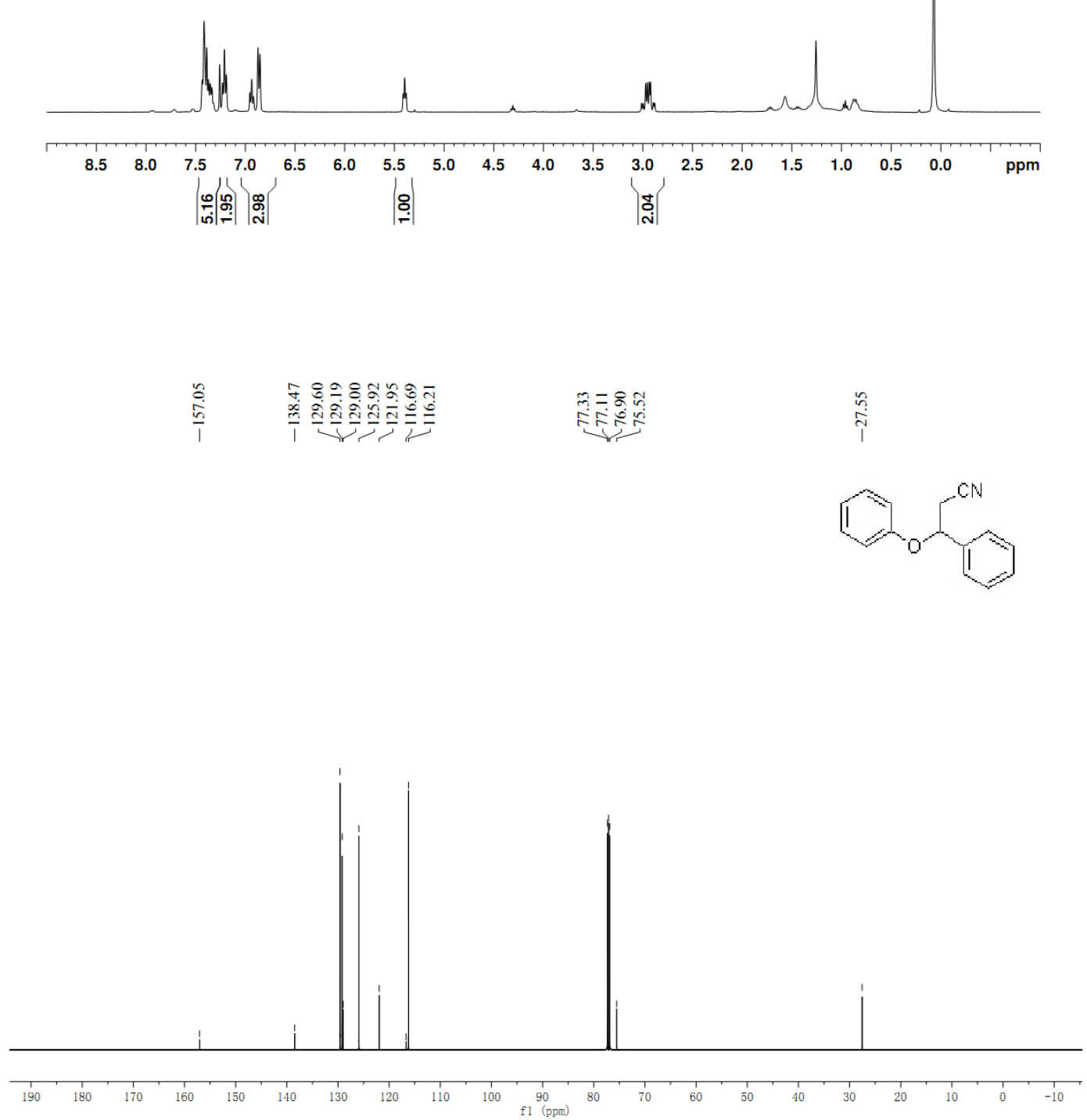

S39 
3-phenyl-3-(o-tolyloxy)propanenitrile (2b)
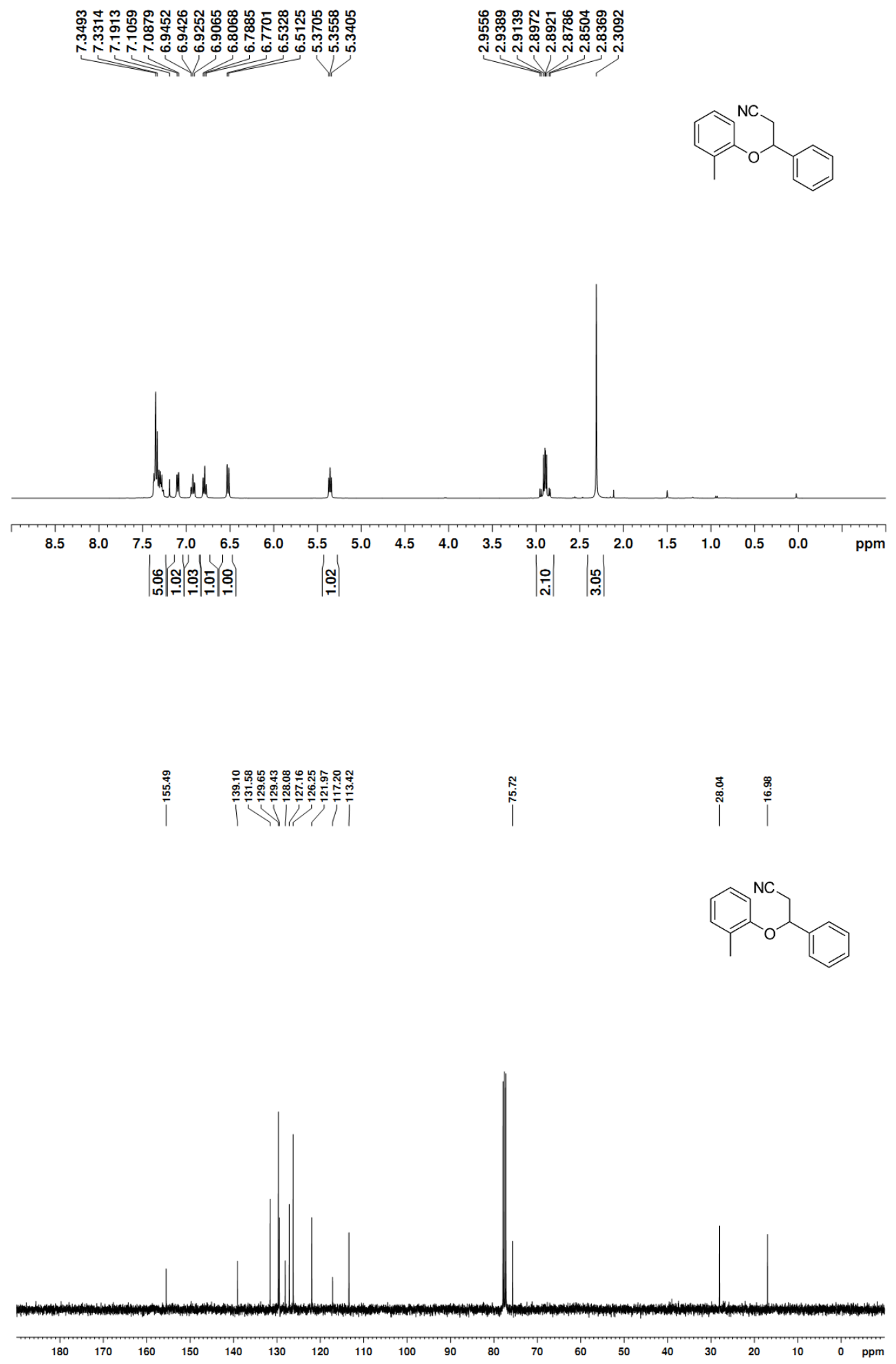

S40 


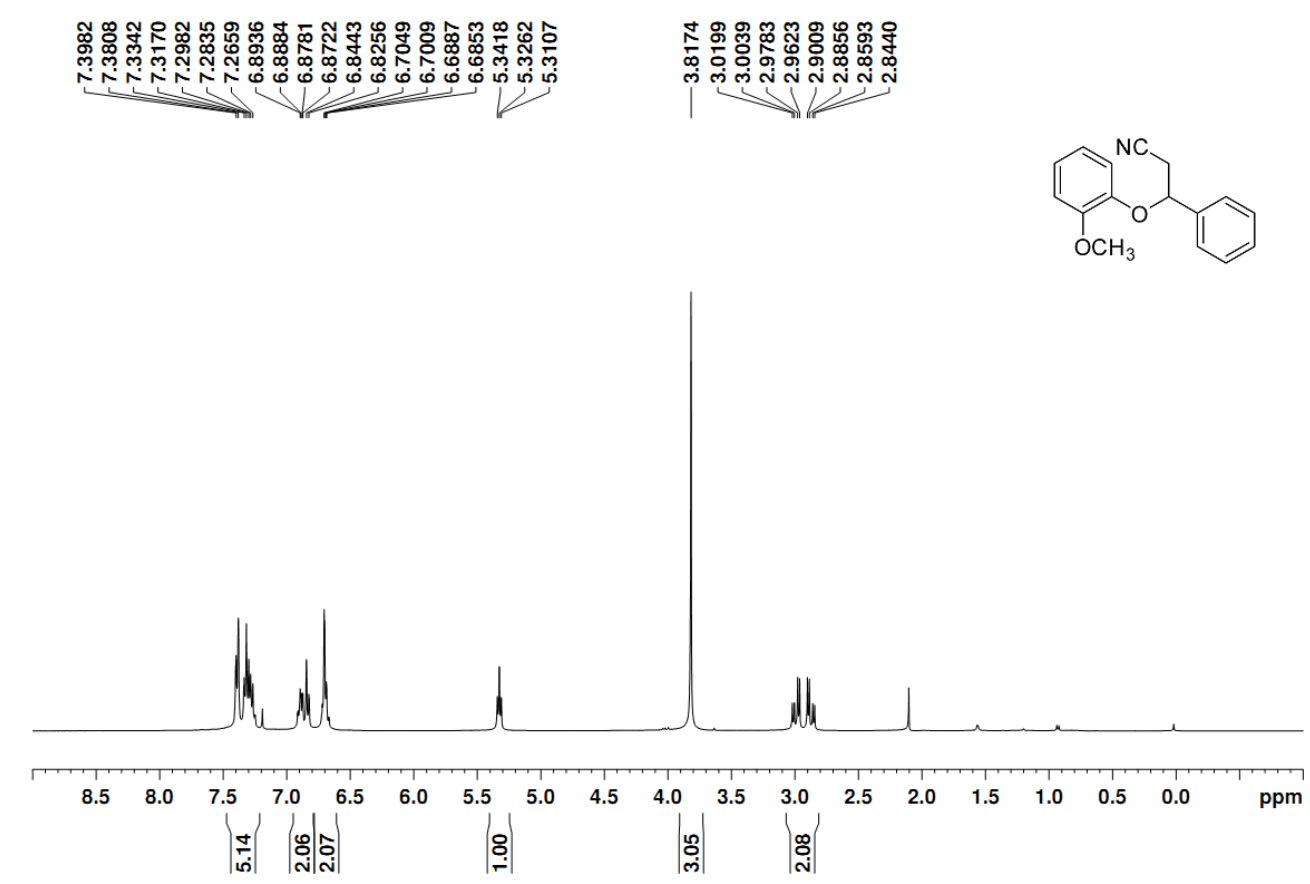

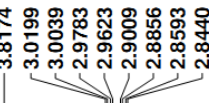

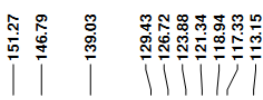
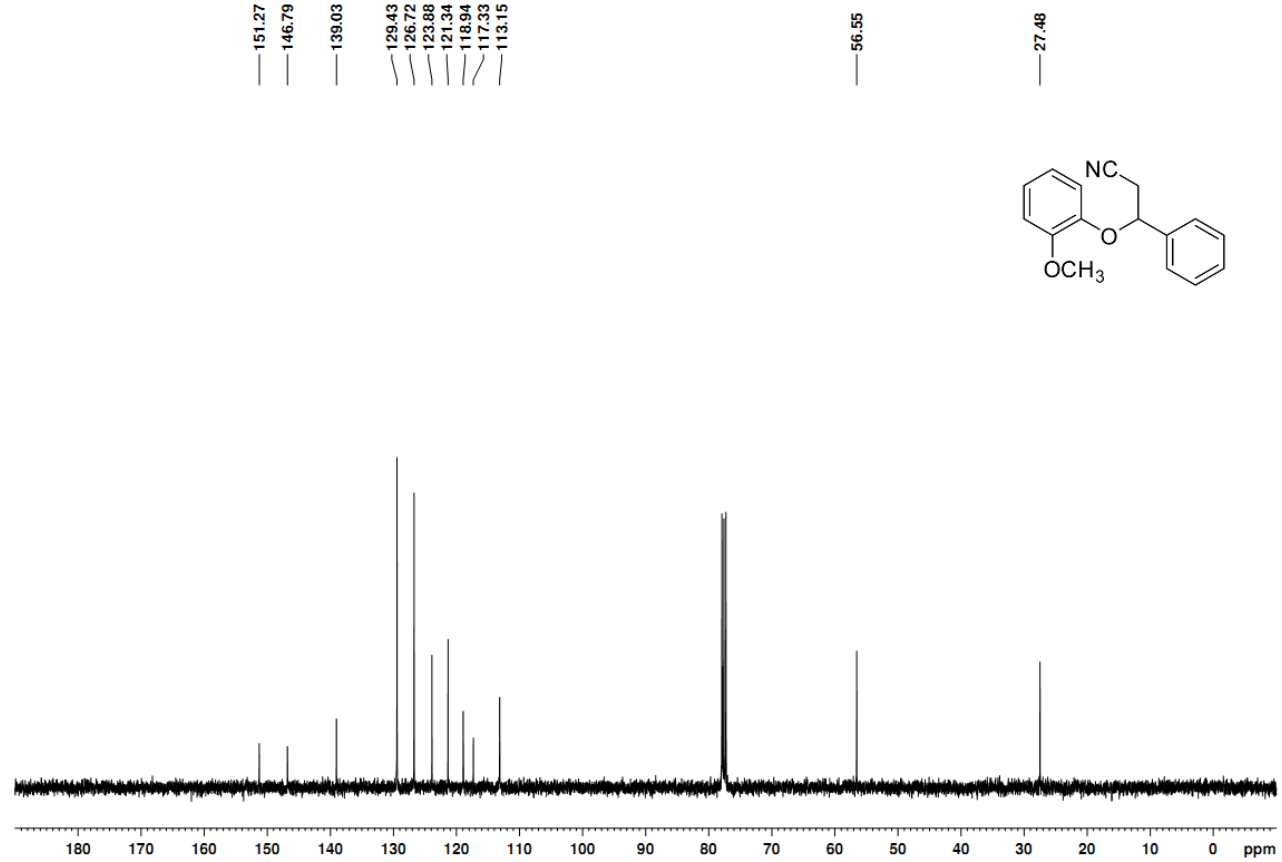

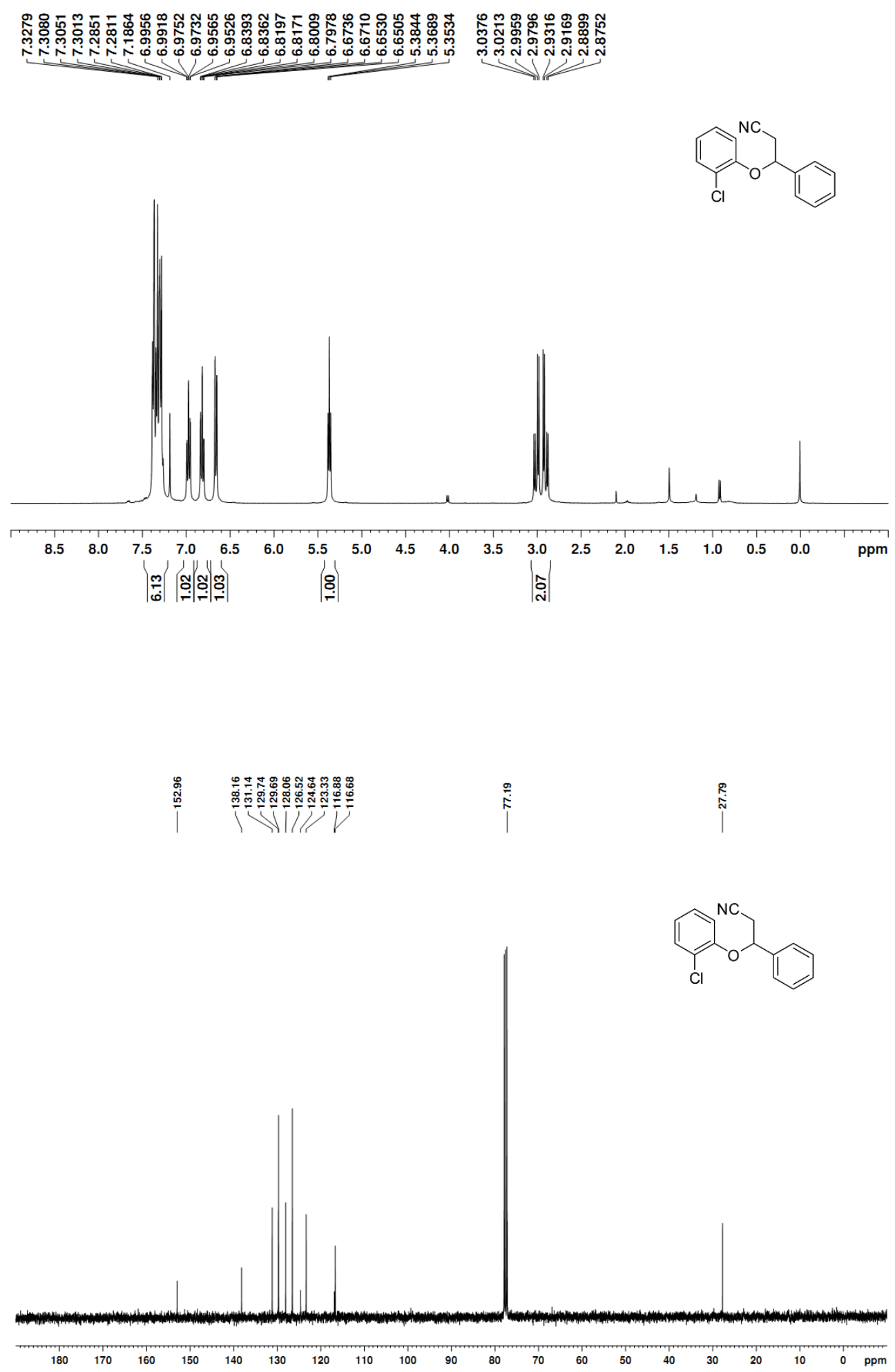


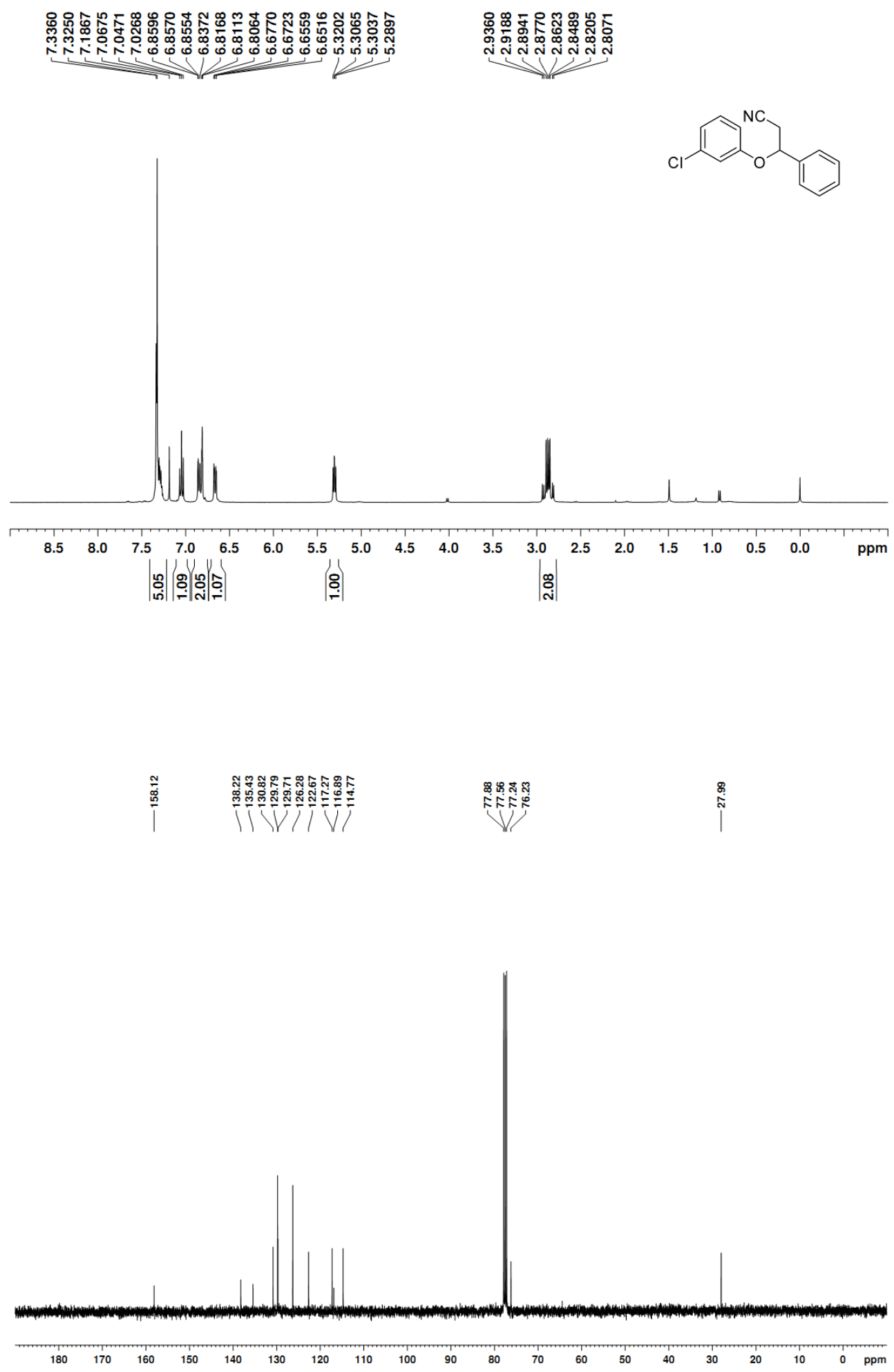


3-phenyl-3-(p-tolyloxy)propanenitrile (2f and 2m)

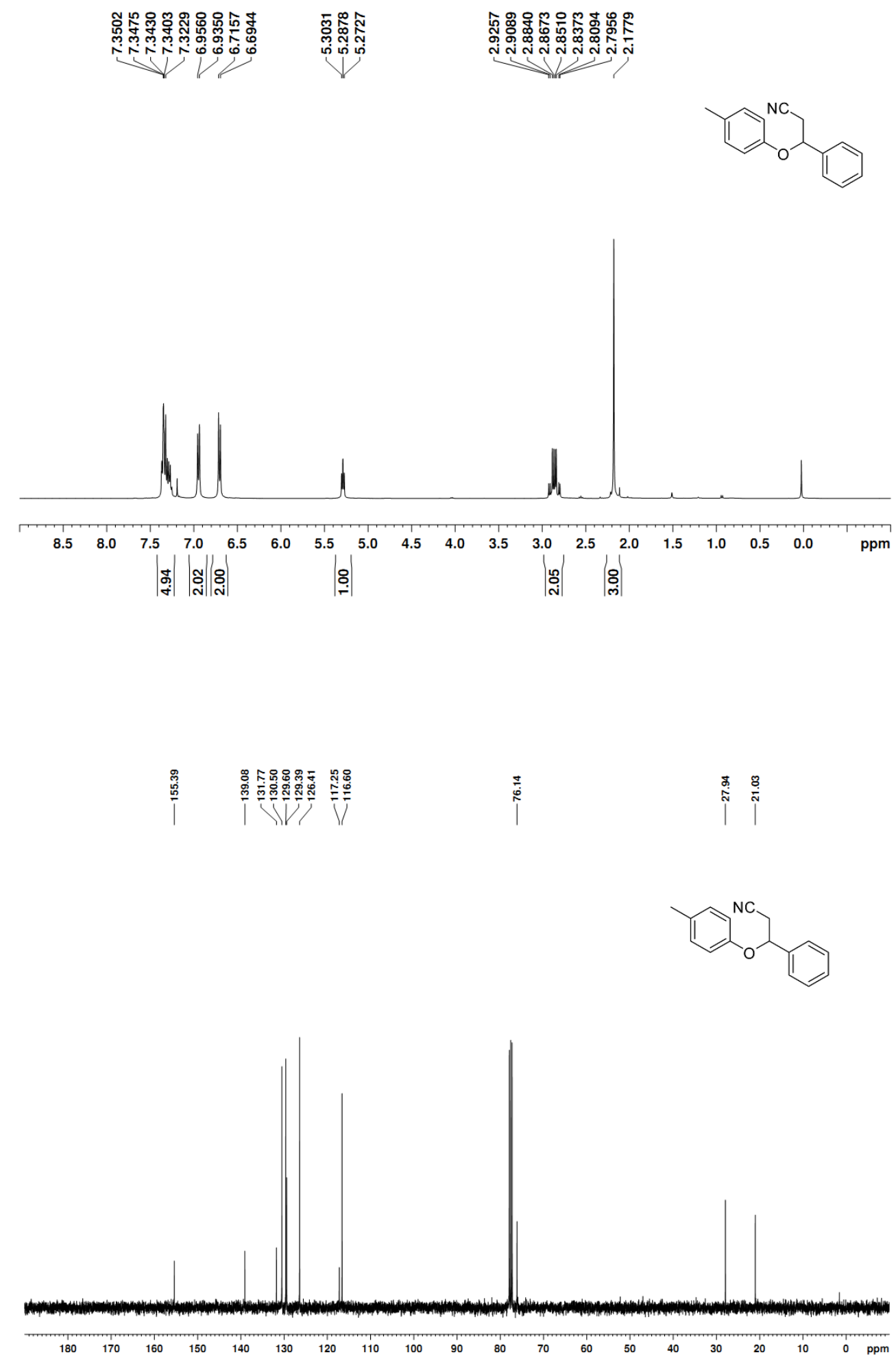


3-(4-chlorophenoxy)-3-phenylpropanenitrile (2g)
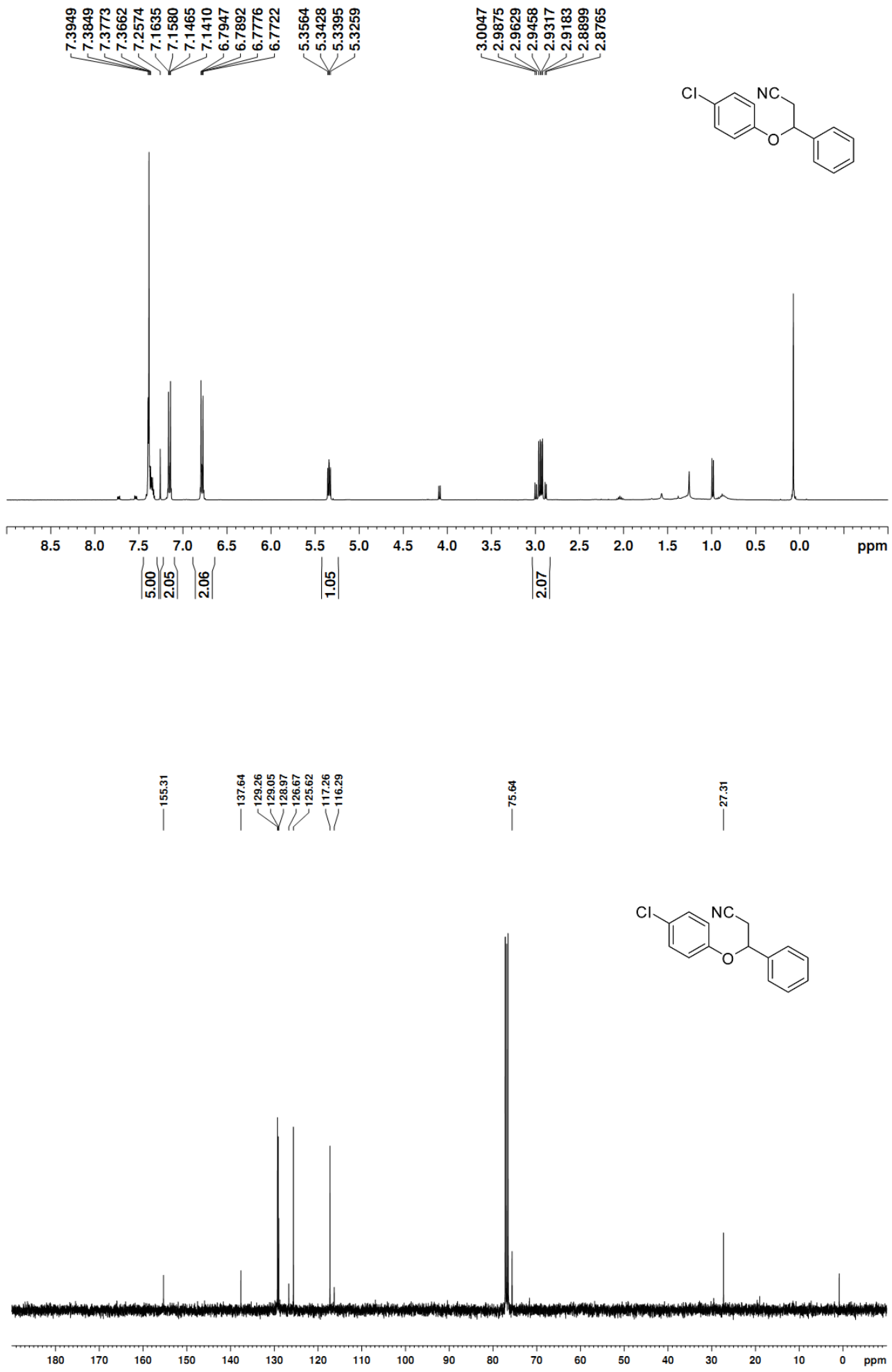

S45 
3-phenyl-3-(4-(trifluoromethyl)phenoxy)propanenitrile (2h)

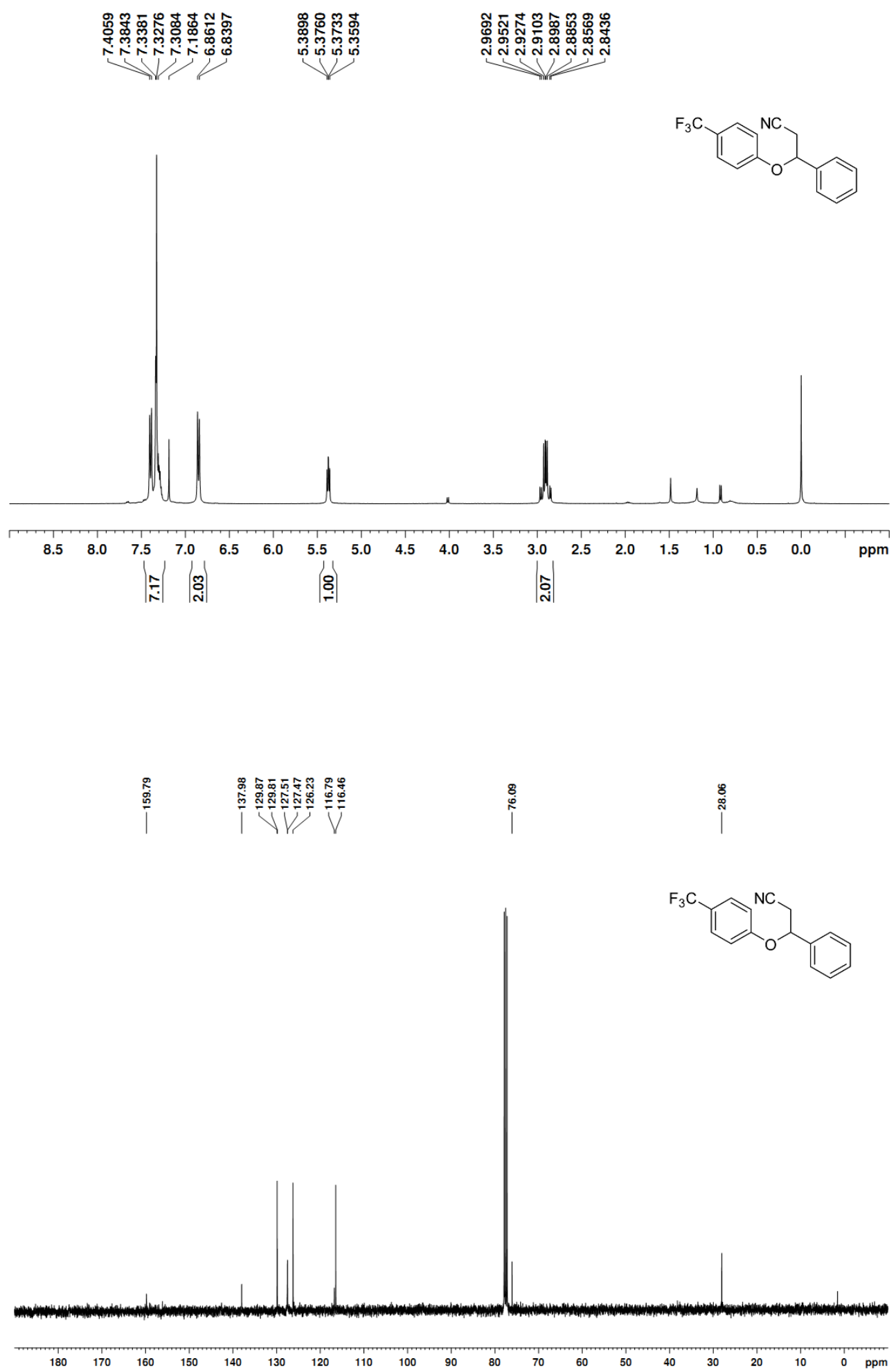


3-(naphthalen-1-yloxy)-3-phenylpropanenitrile (2i)

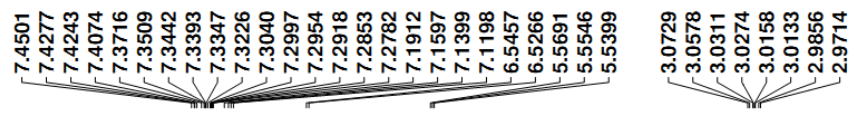
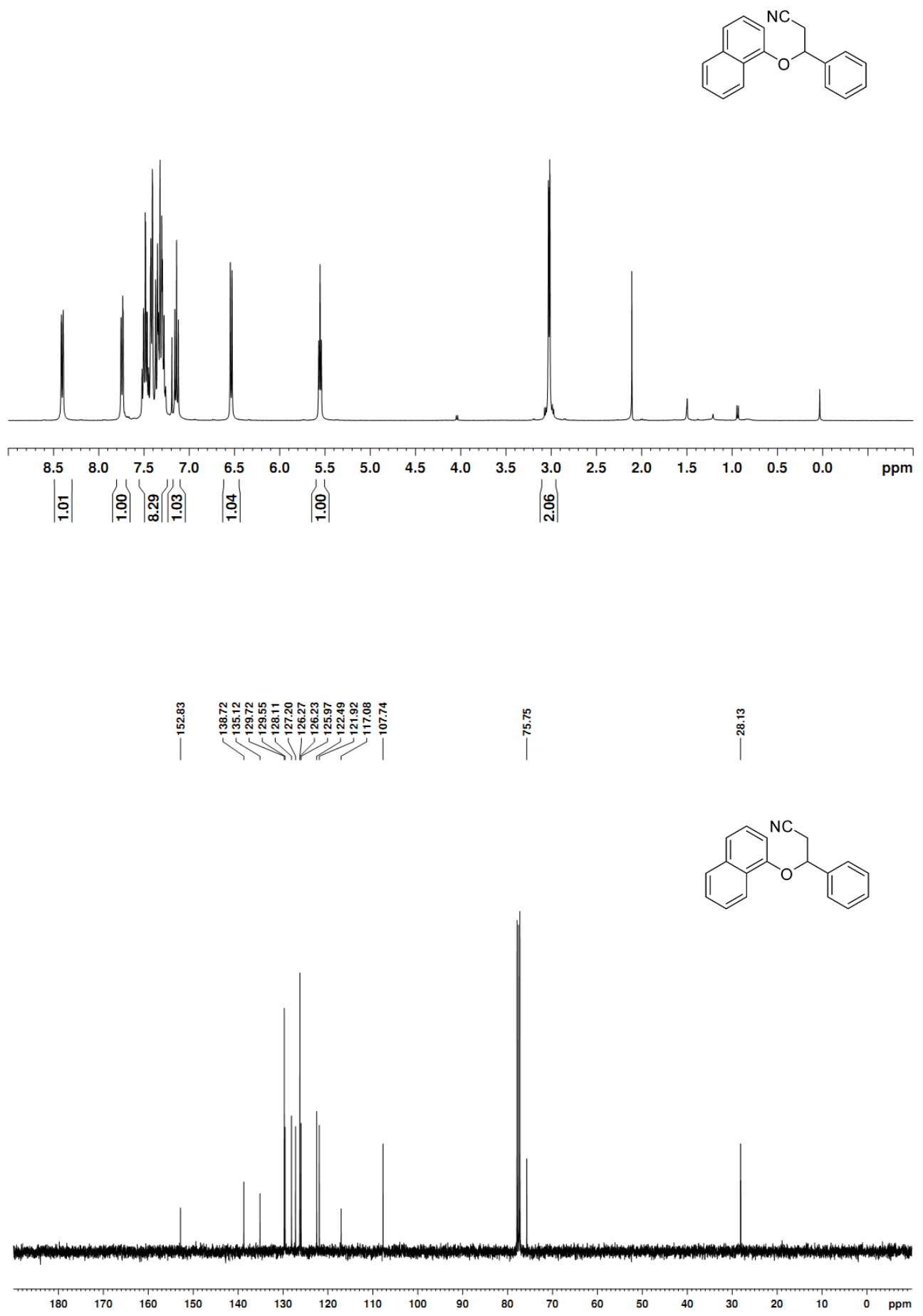

S47 
3-(naphthalen-2-yloxy)-3-phenylpropanenitrile (2j)

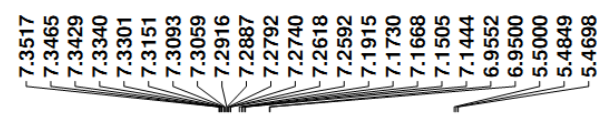

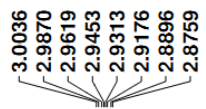
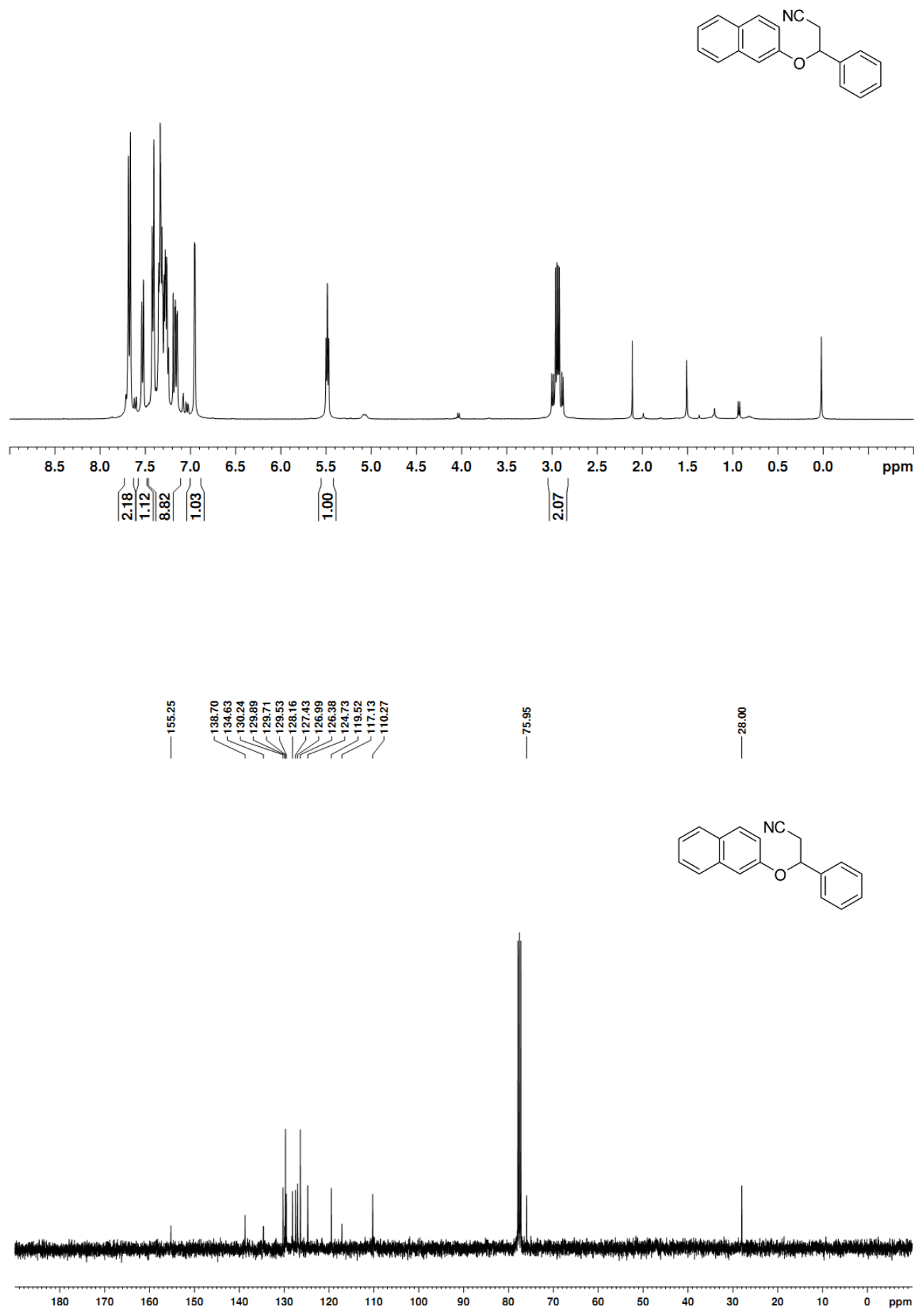

S48 
3-methoxy-3-phenylpropanenitrile(2n)
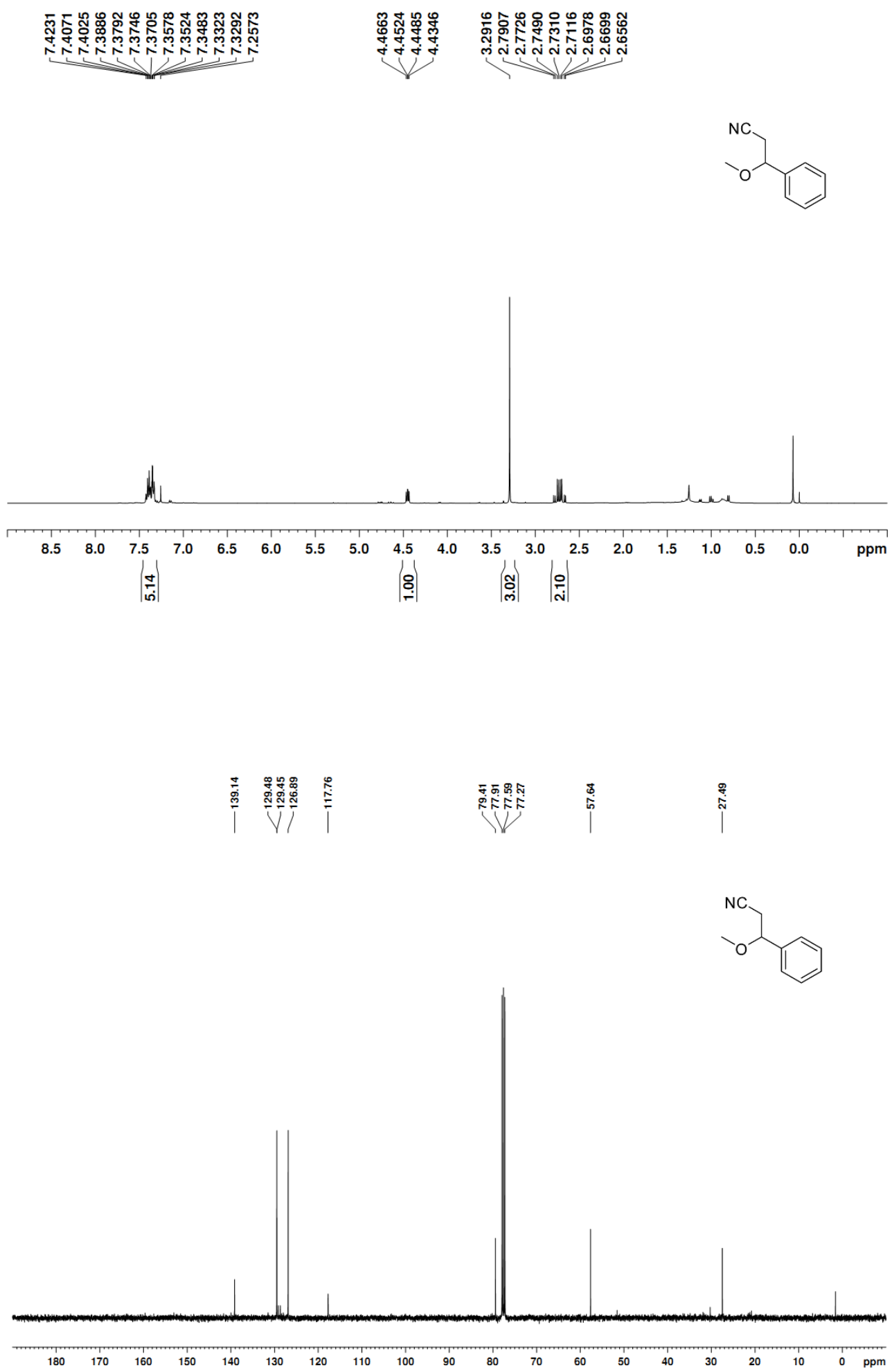
3-isopropoxy-3-phenylpropanenitrile (2o)

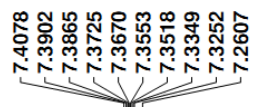
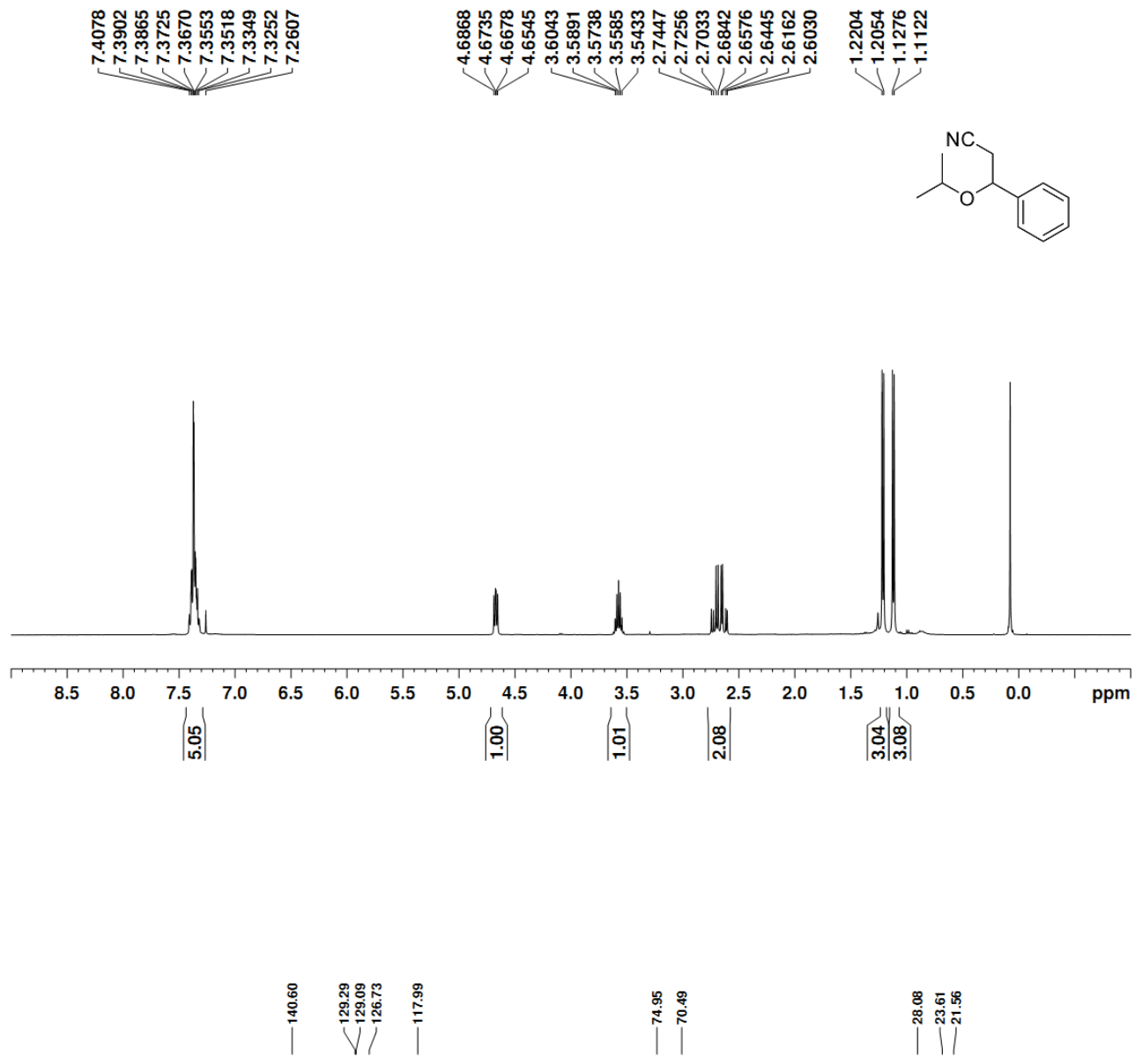

影
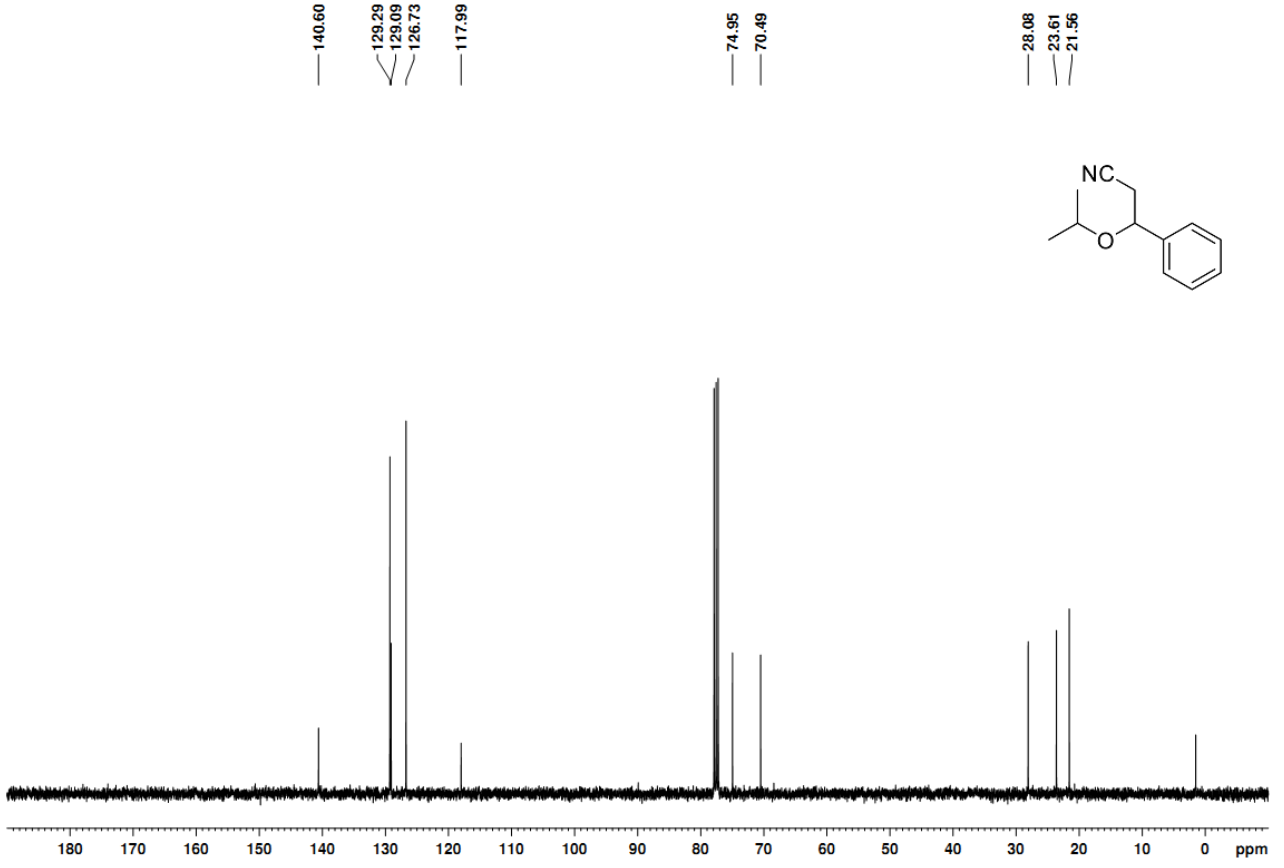

S50 
3-phenyl-3-(2,2,2-trifluoroethoxy)propanenitrile (2p)

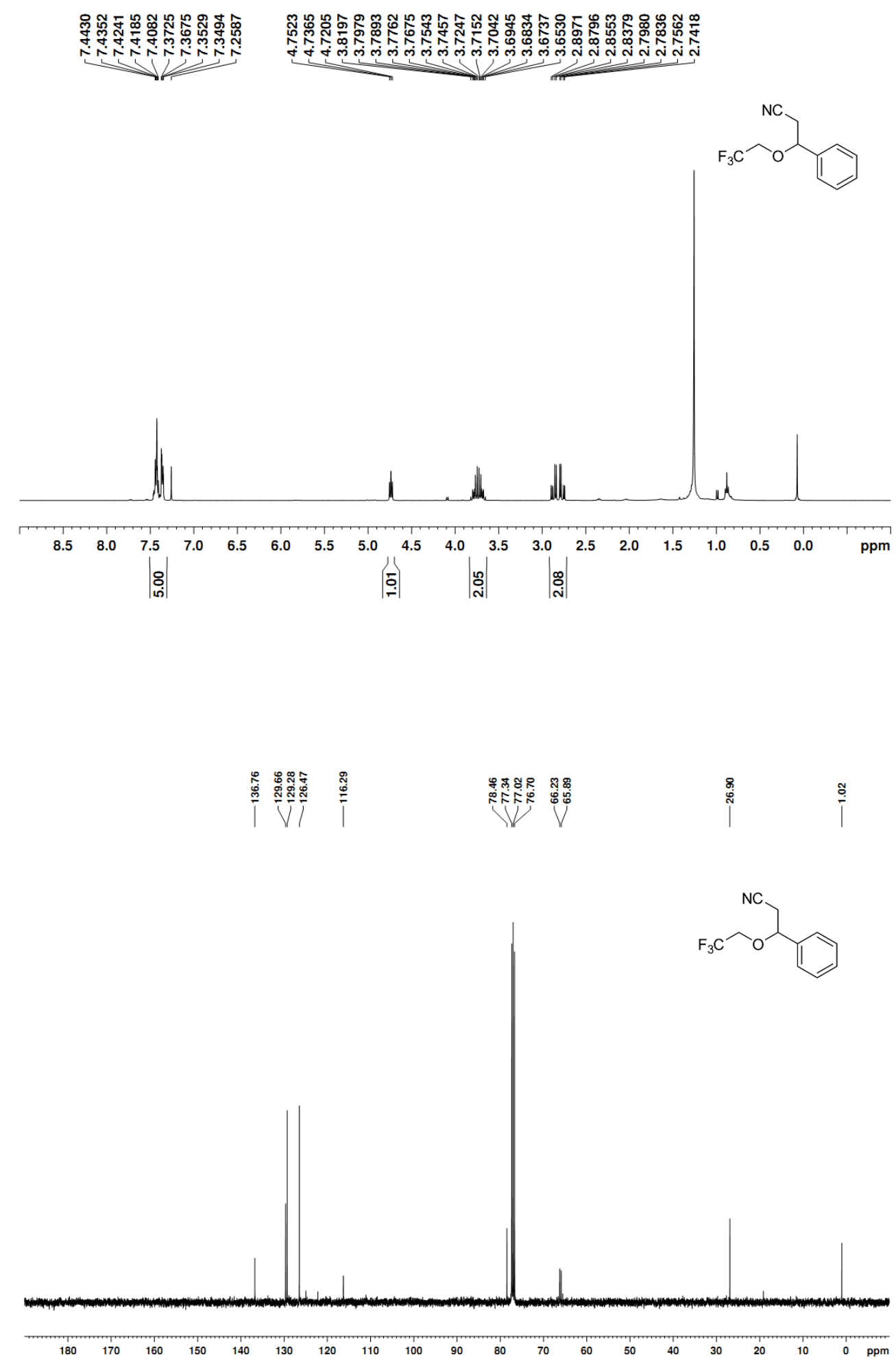


3-phenyl-3-(1H-pyrrol-1-yl)propanenitrile (2q)
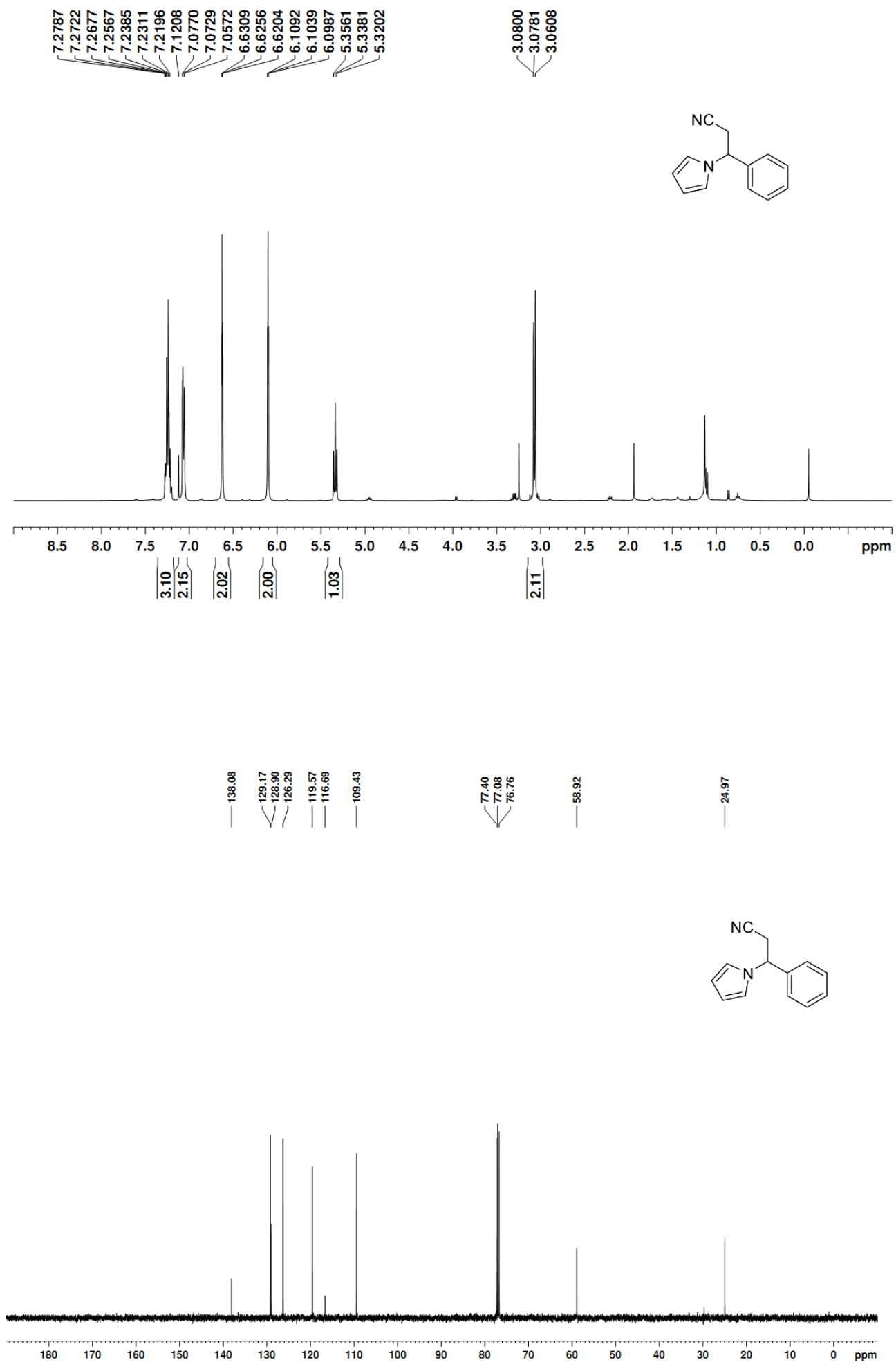
dimethyl (2-cyano-1-(p-tolyl)ethyl)phosphonate (2r)

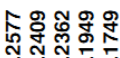

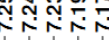

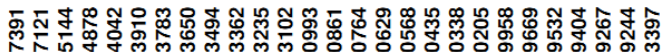

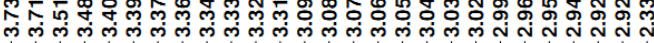
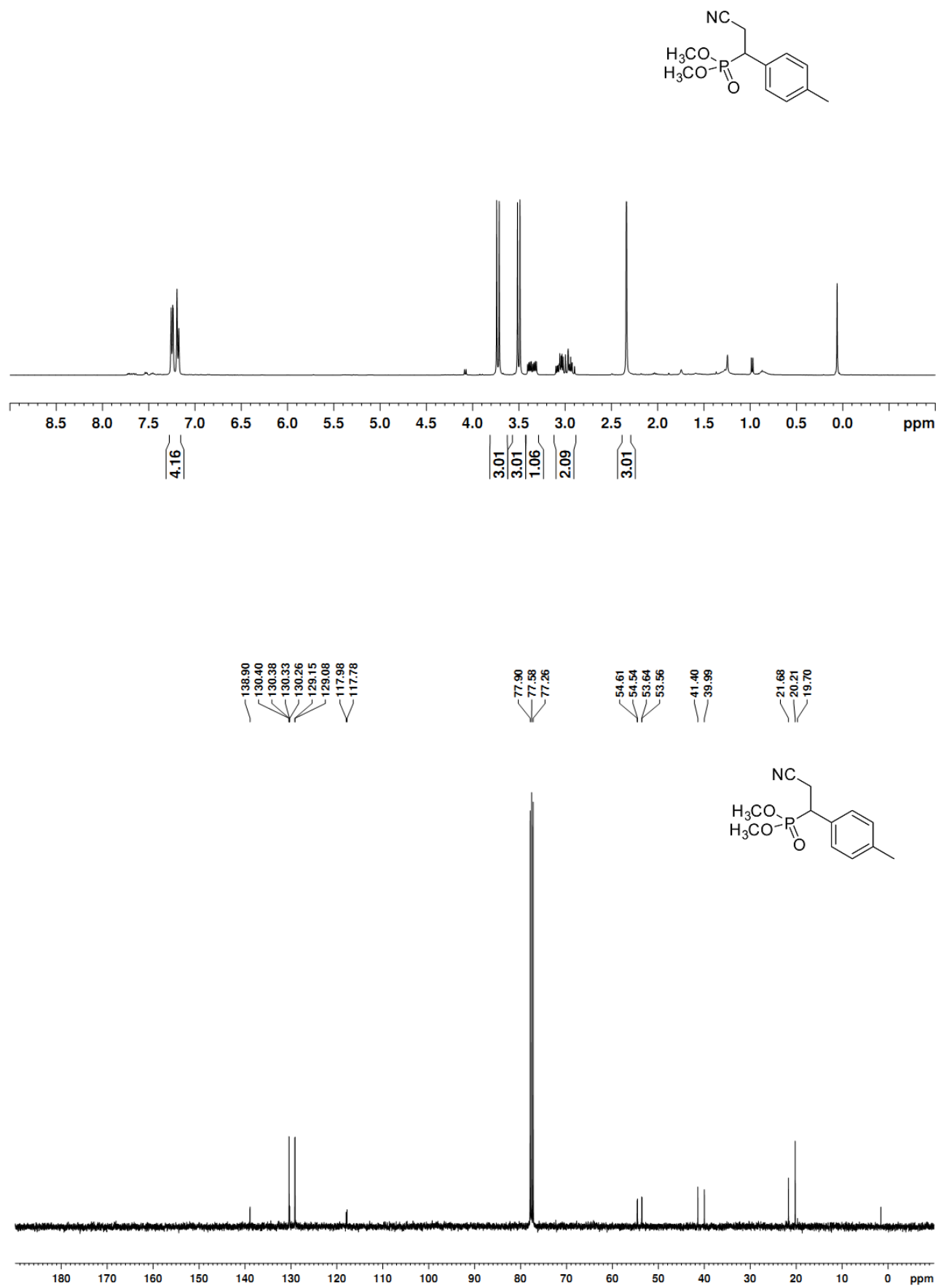
(Z)-methyl 3-phenoxy-3-phenylacrylate (3a)

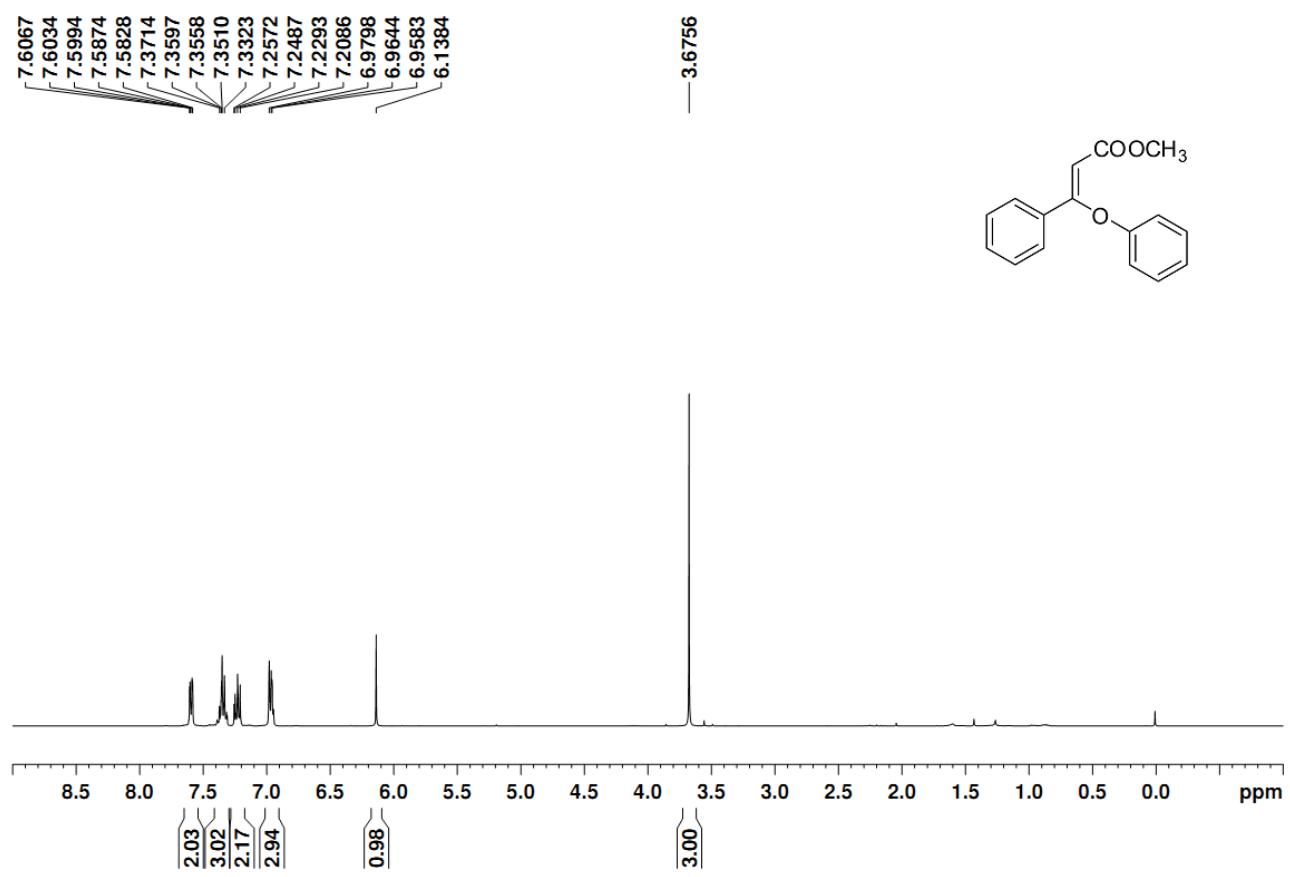

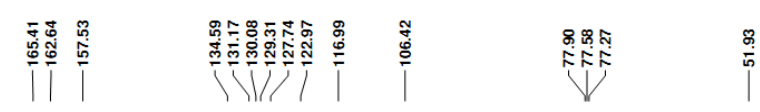
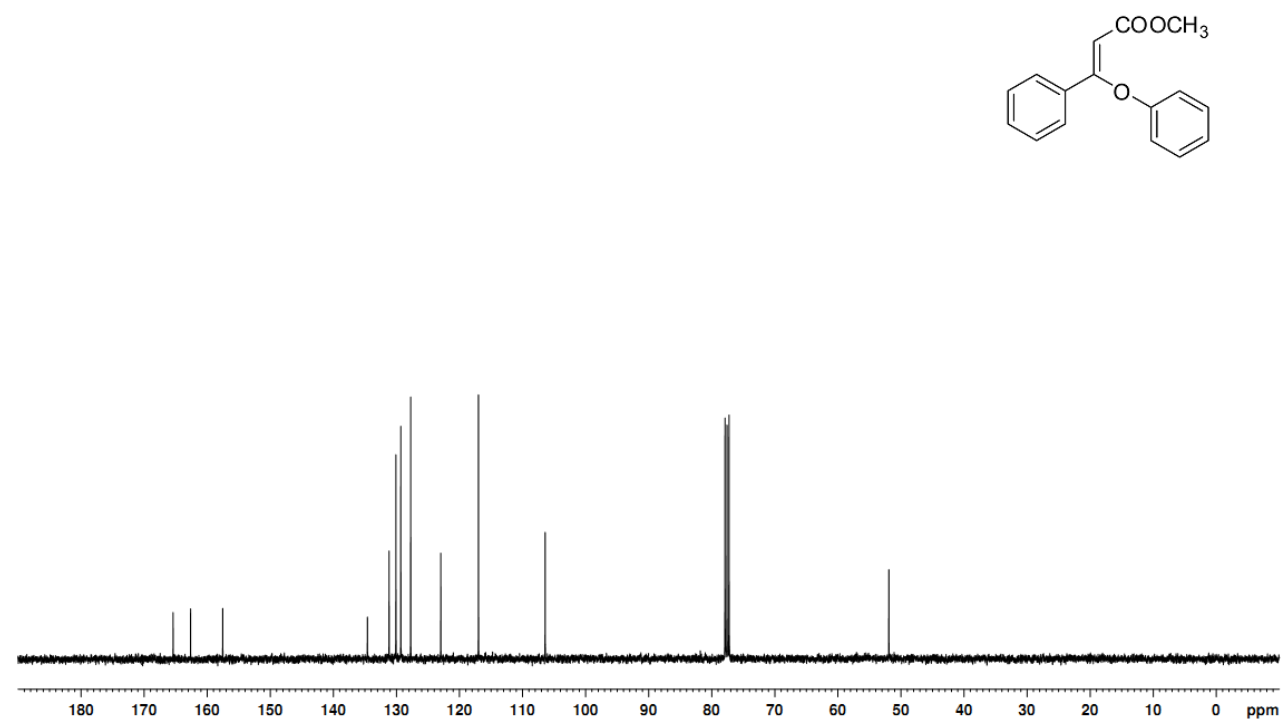


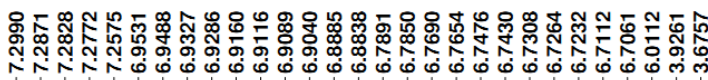

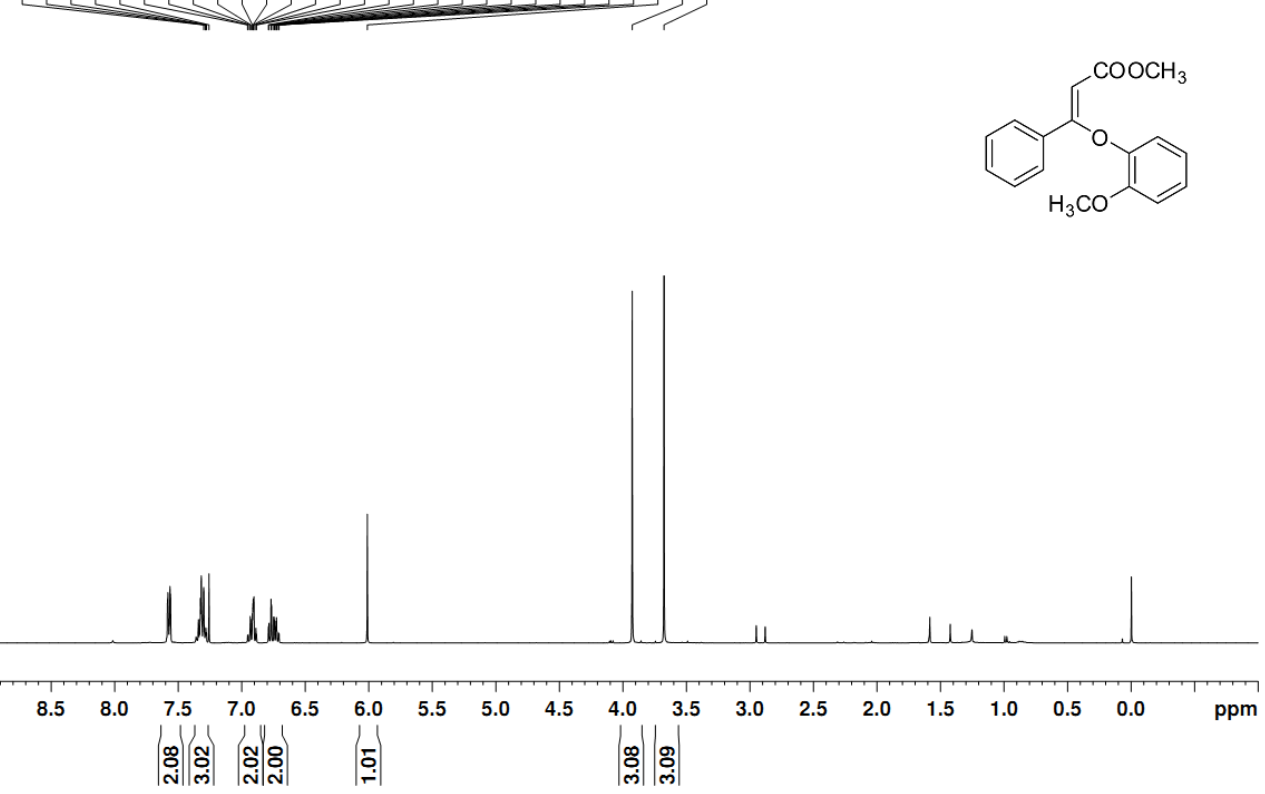

li

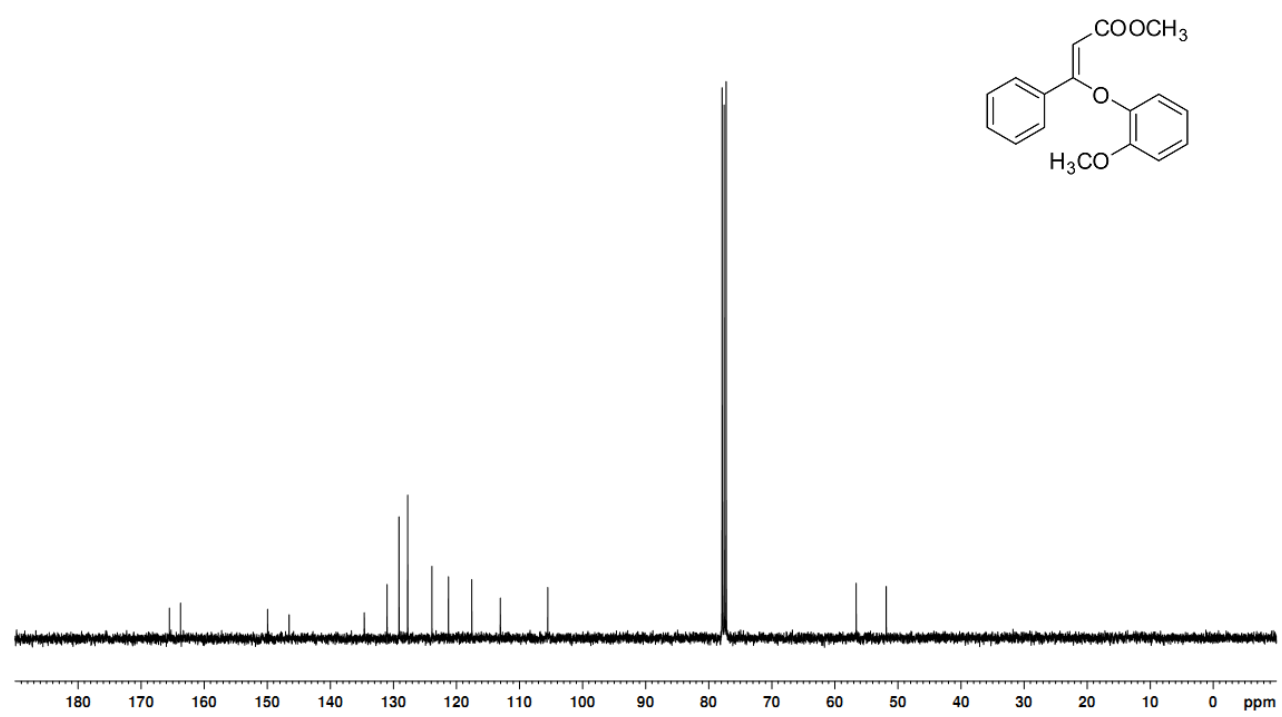


(Z)-methyl 3-phenyl-3-(o-tolyloxy)acrylate (3c)

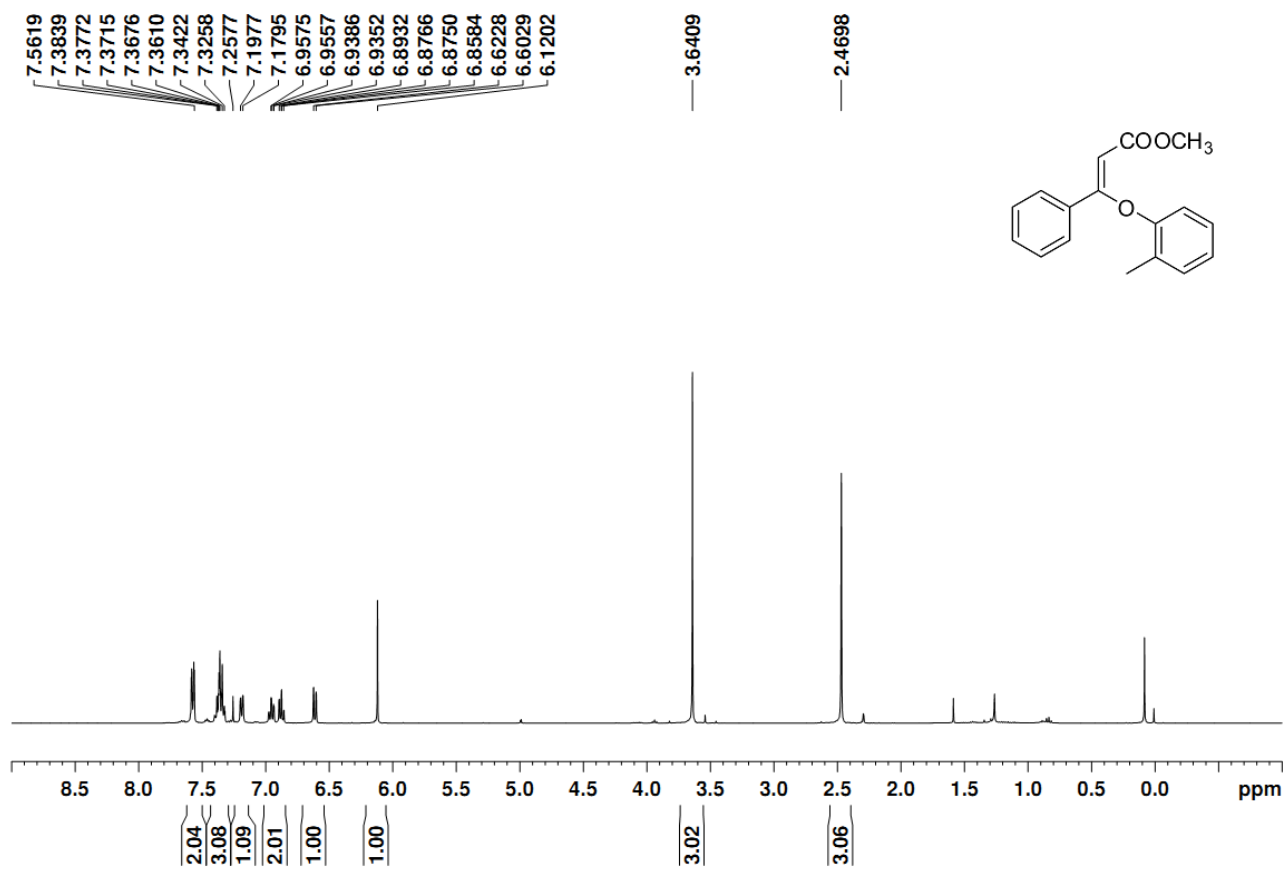

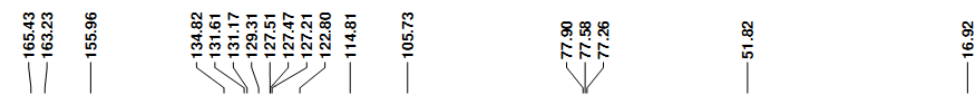

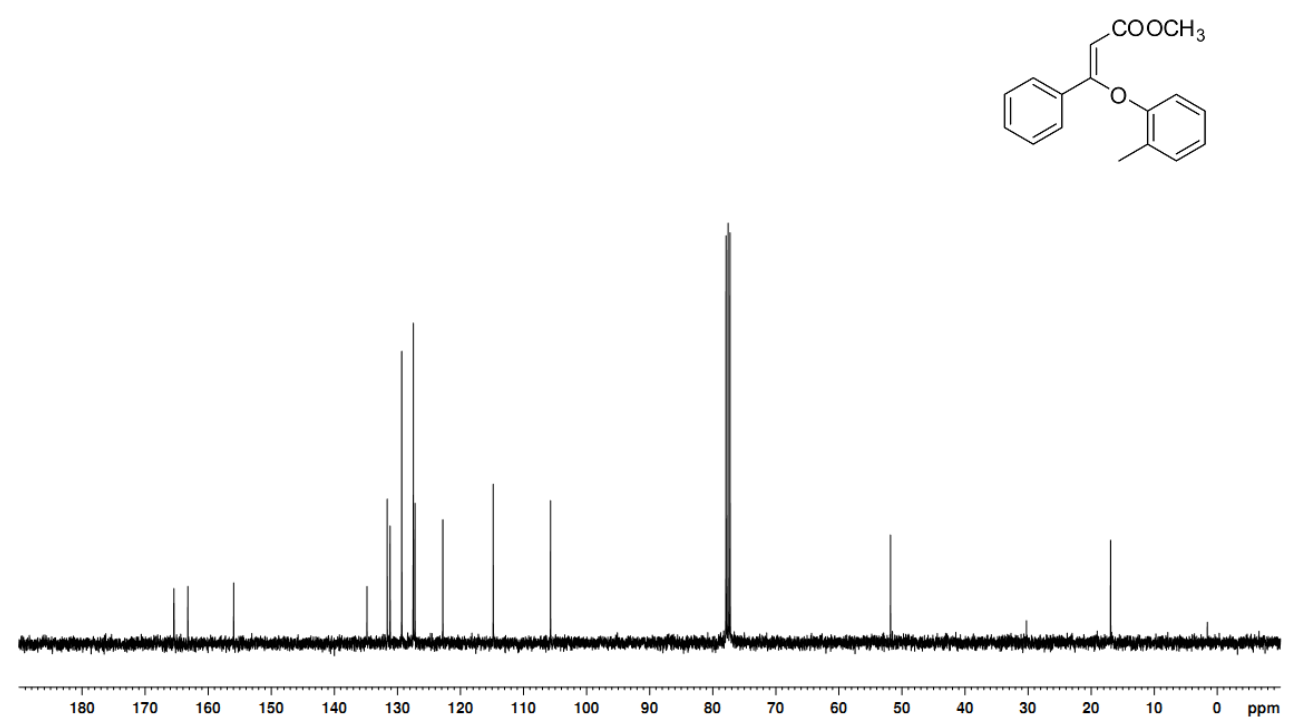


(Z)-methyl 3-phenyl-3-(p-tolyloxy)acrylate (3d)

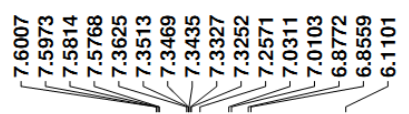
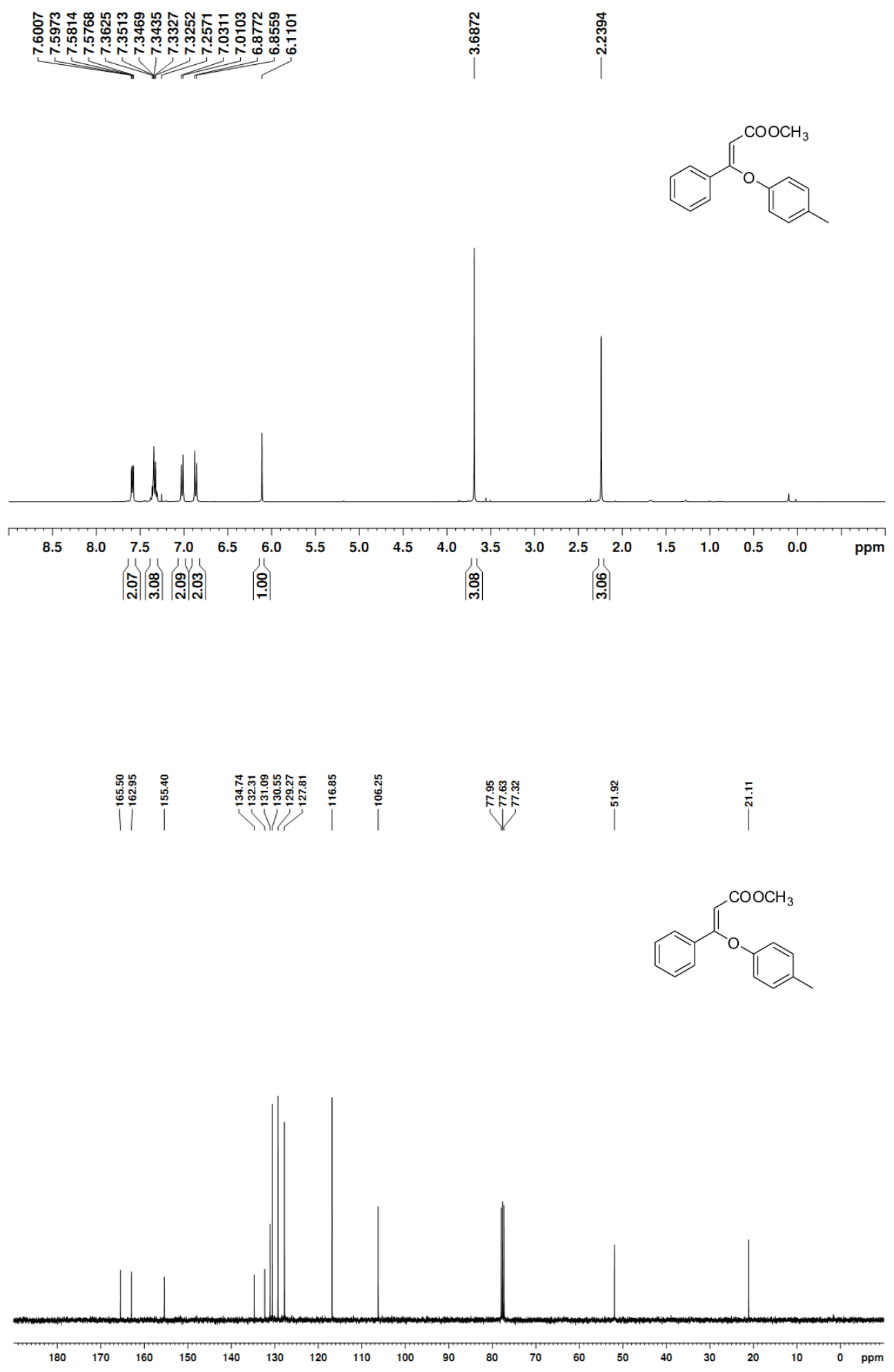

S57 
(E)-methyl 3-phenyl-3-(p-tolyloxy)acrylate (3e)

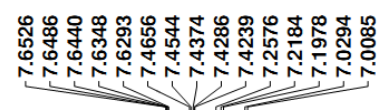

$\underset{\substack{0 \\ 0}}{0}$

高

\}
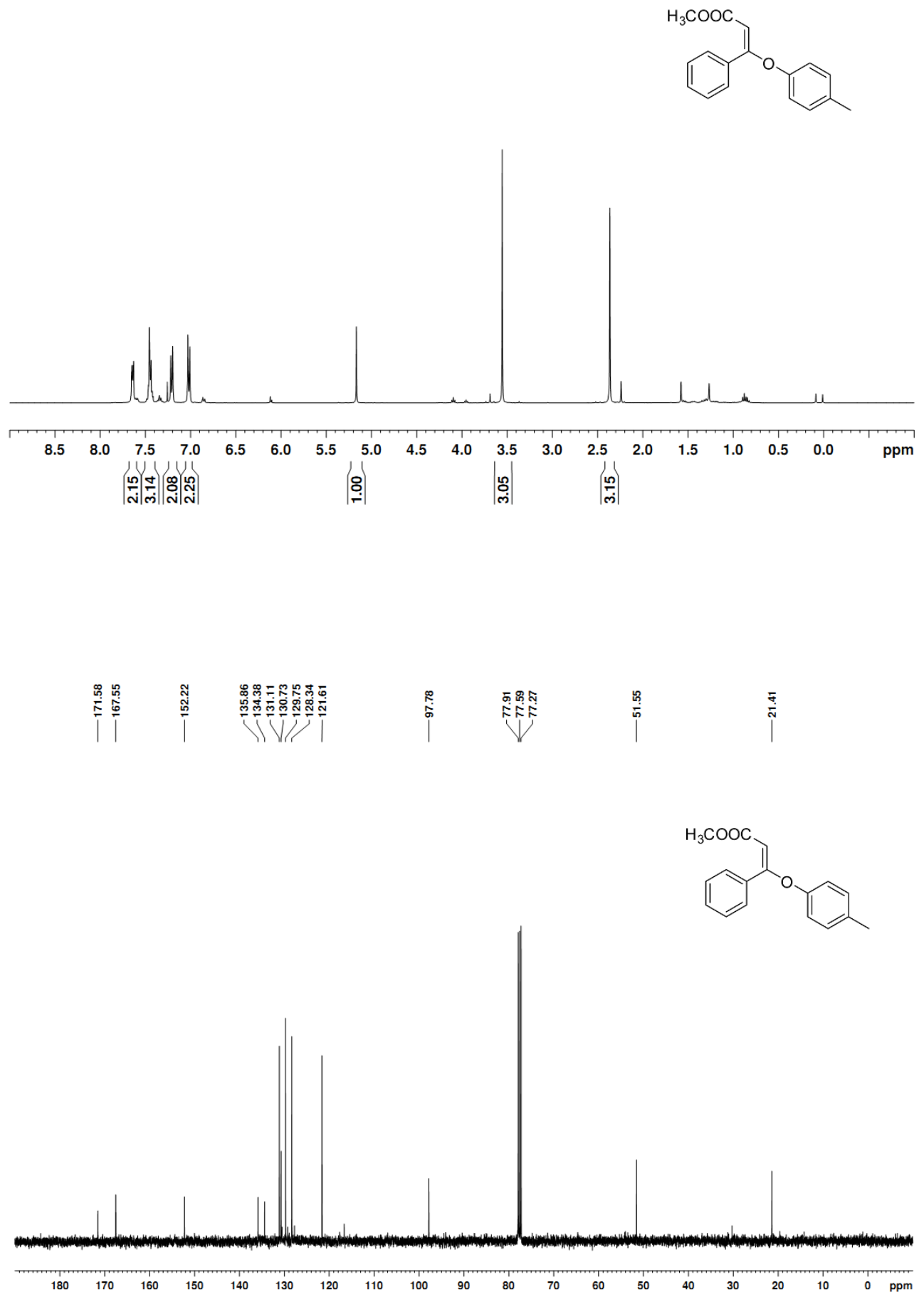

S58 
(E)-methyl 3-(4-chlorophenoxy)-3-phenylacrylate (3f)
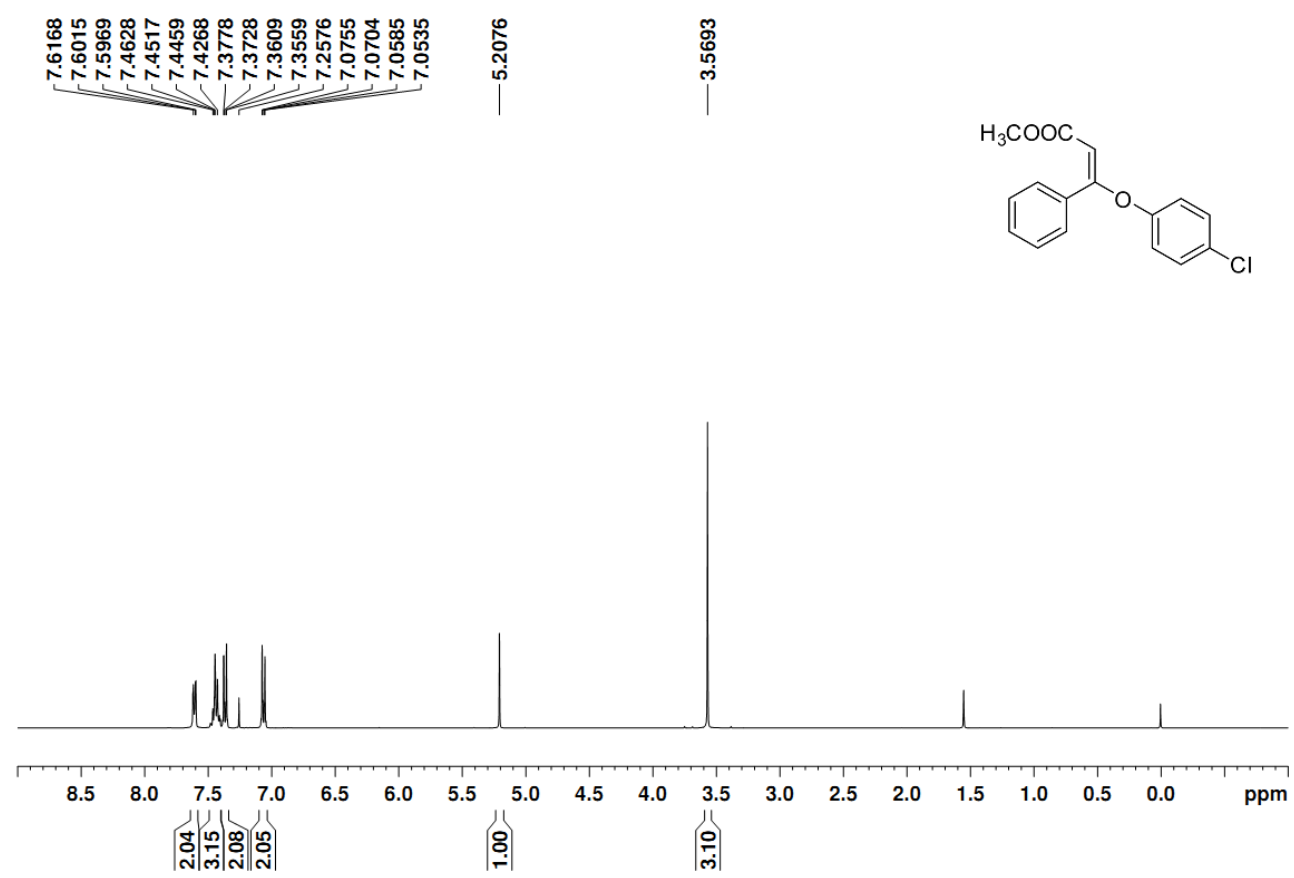

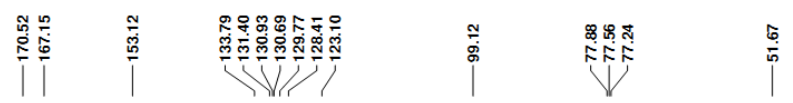

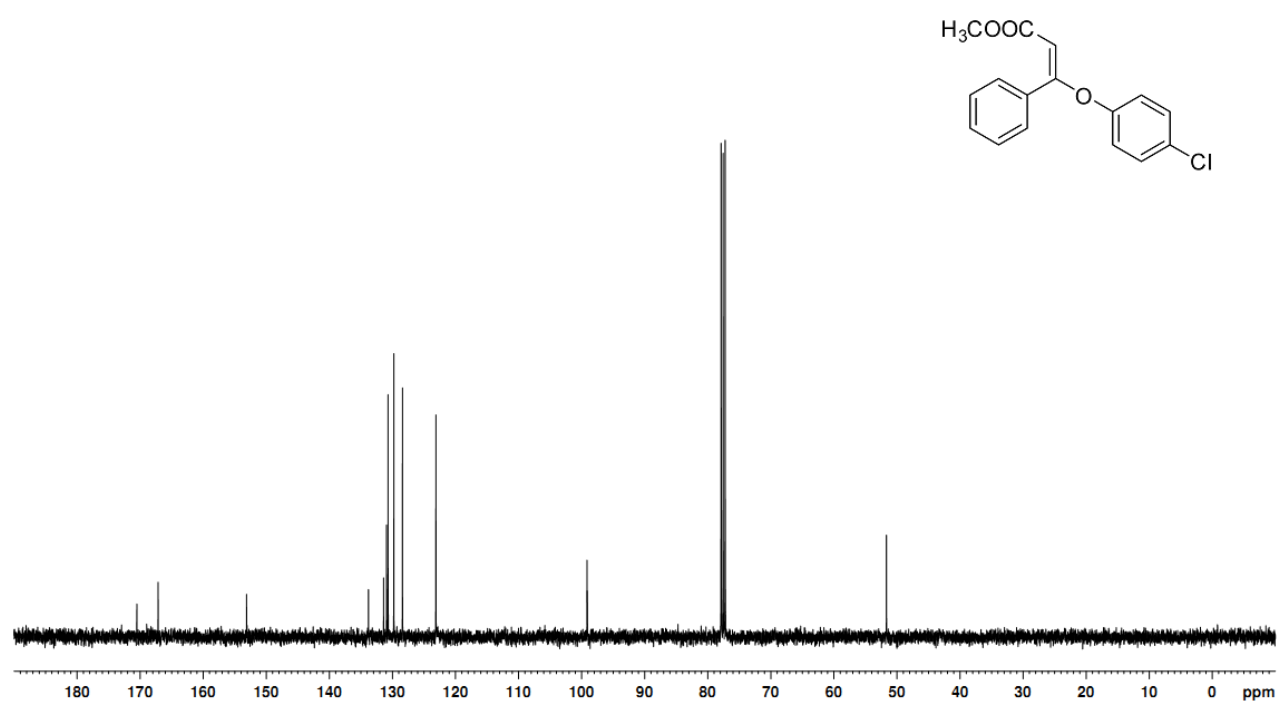


(E)-ethyl 3-phenoxybut-2-enoate (3g)

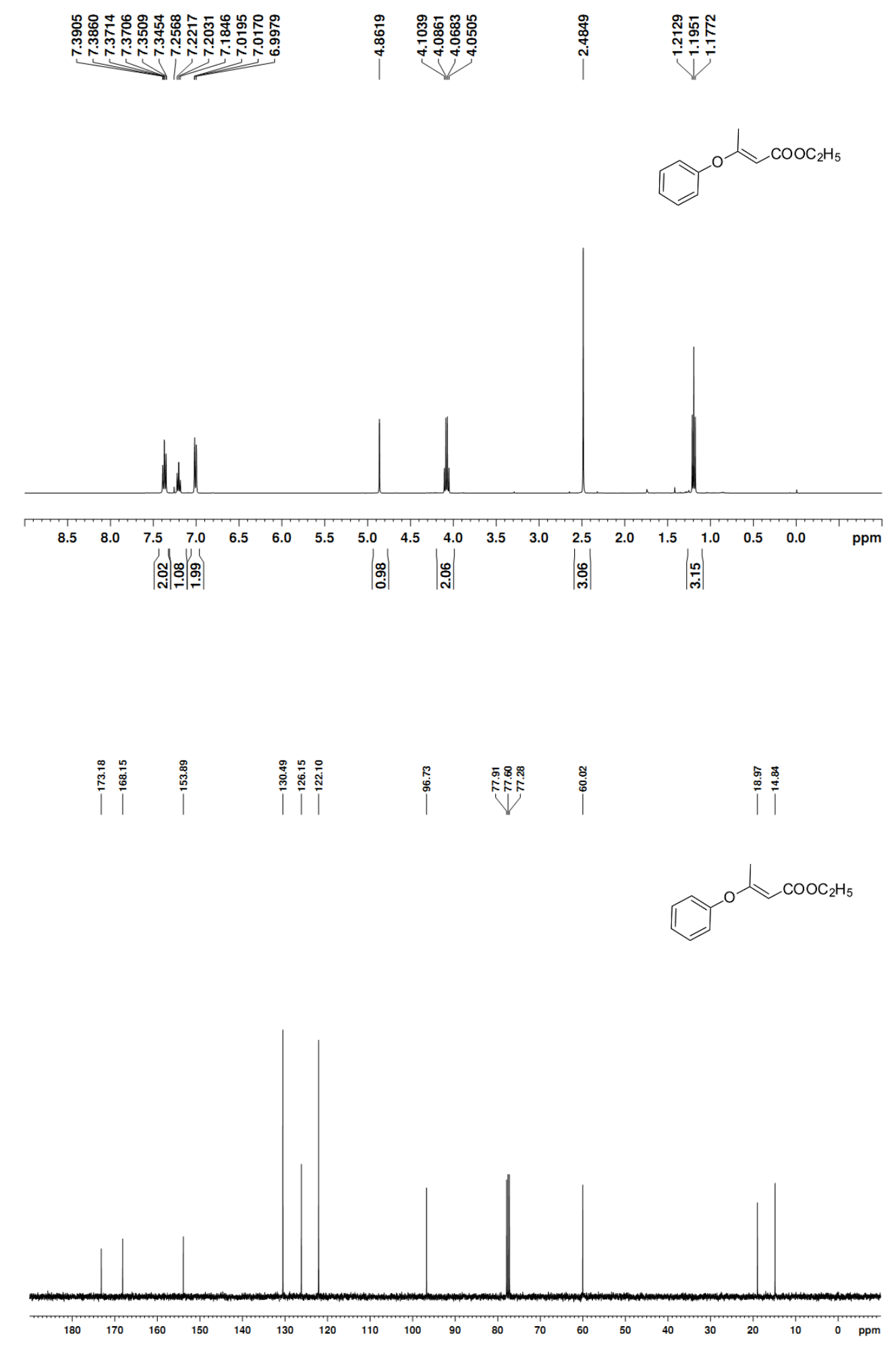


methyl 3-phenoxy-3-phenylpropanoate (4a)

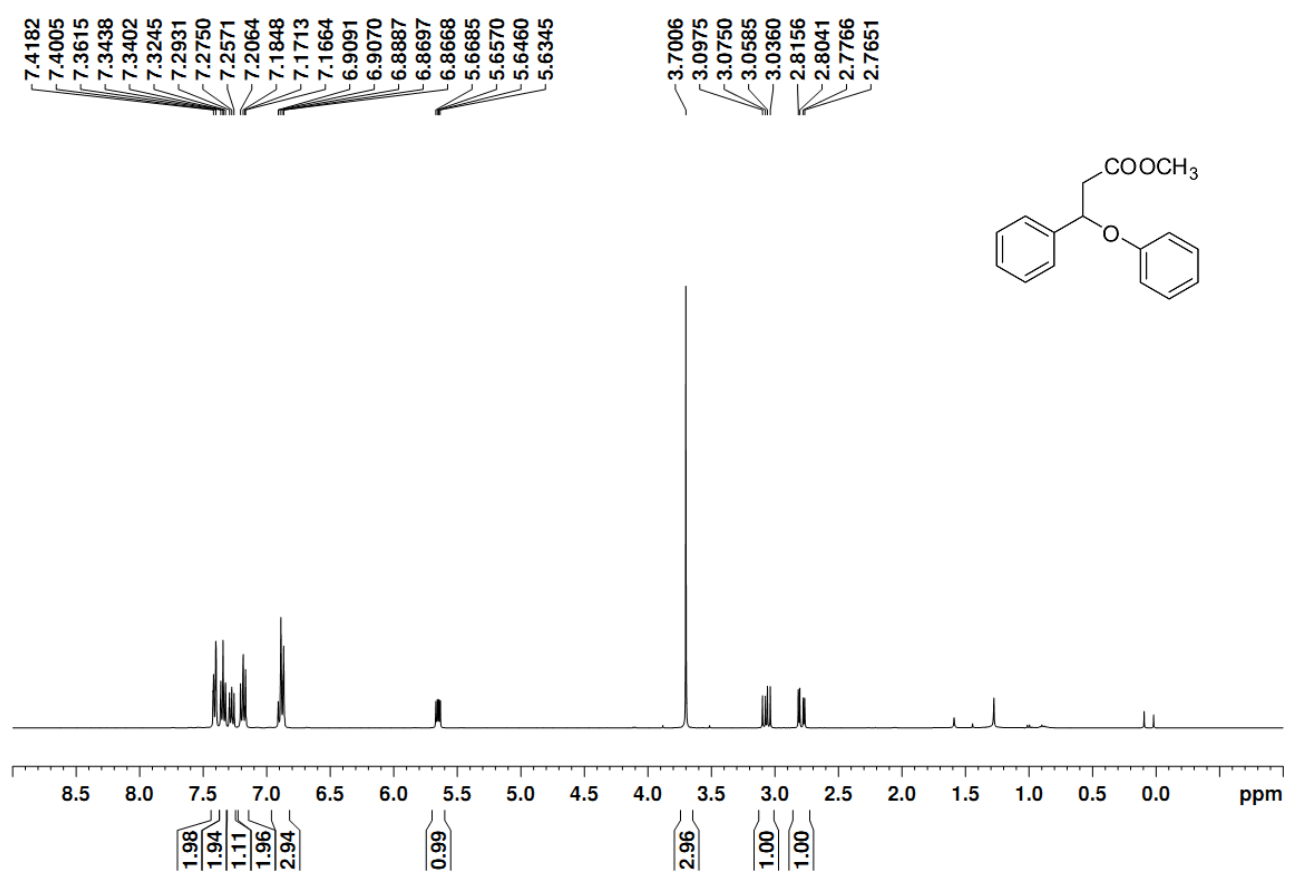

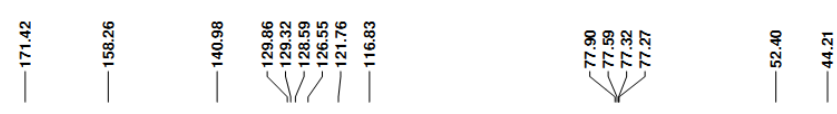
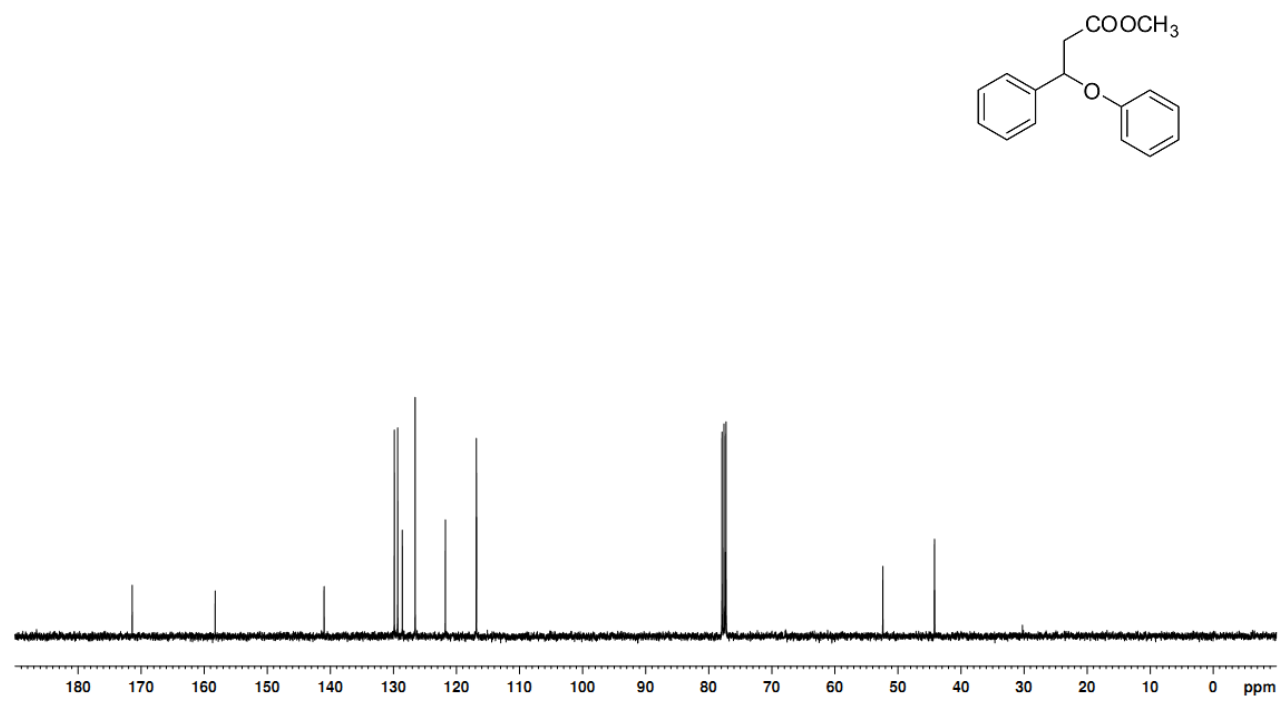
methyl 3-(2-methoxyphenoxy)-3-phenylpropanoate (4b)

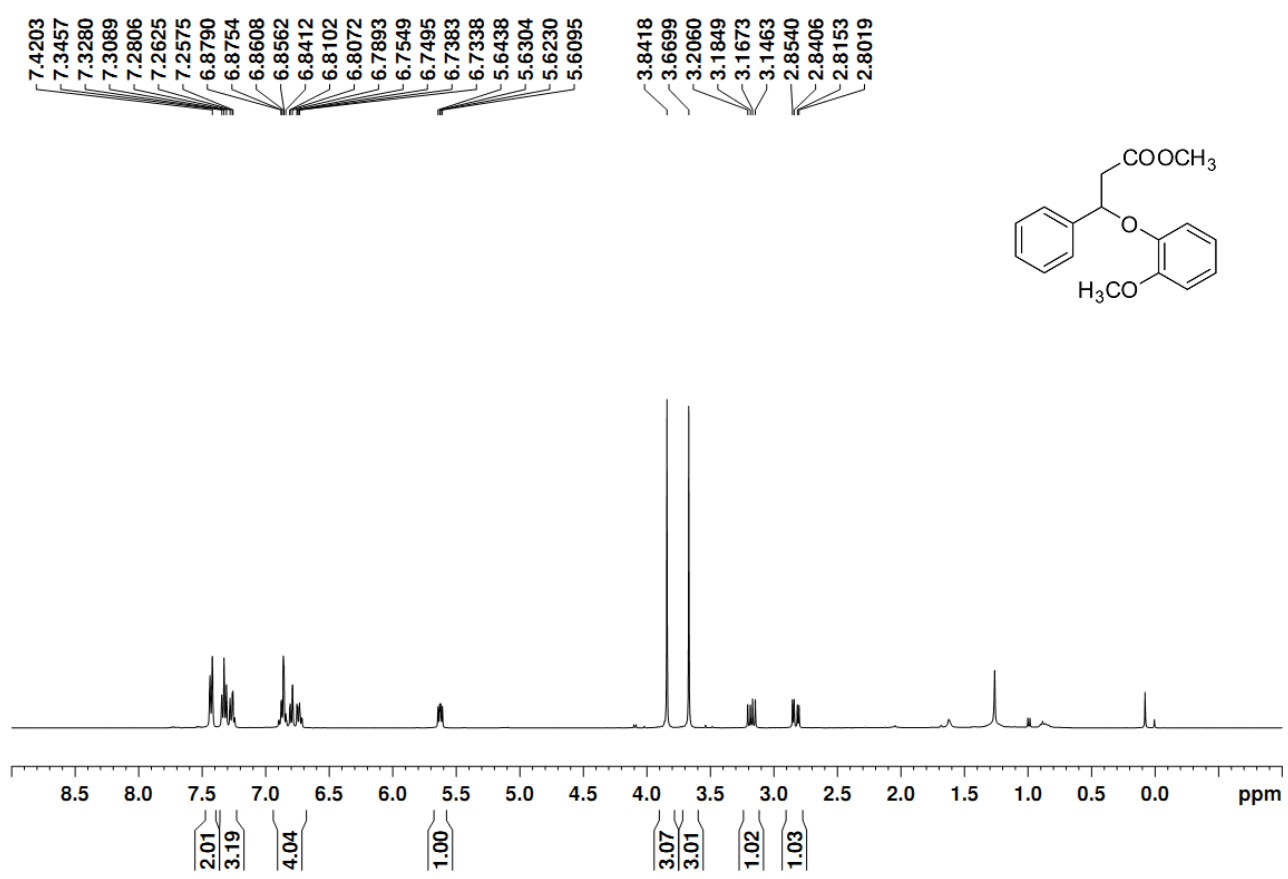

兽
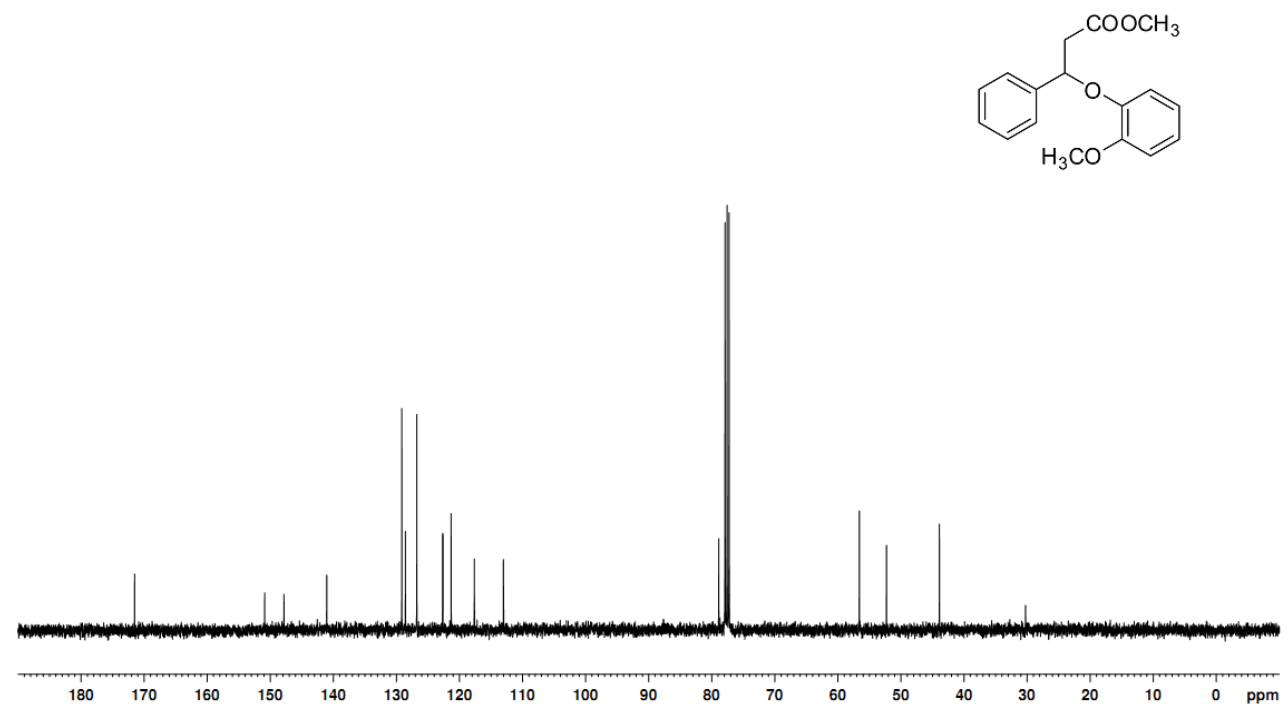
methyl 3-phenyl-3-(o-tolyloxy)propanoate (4c)
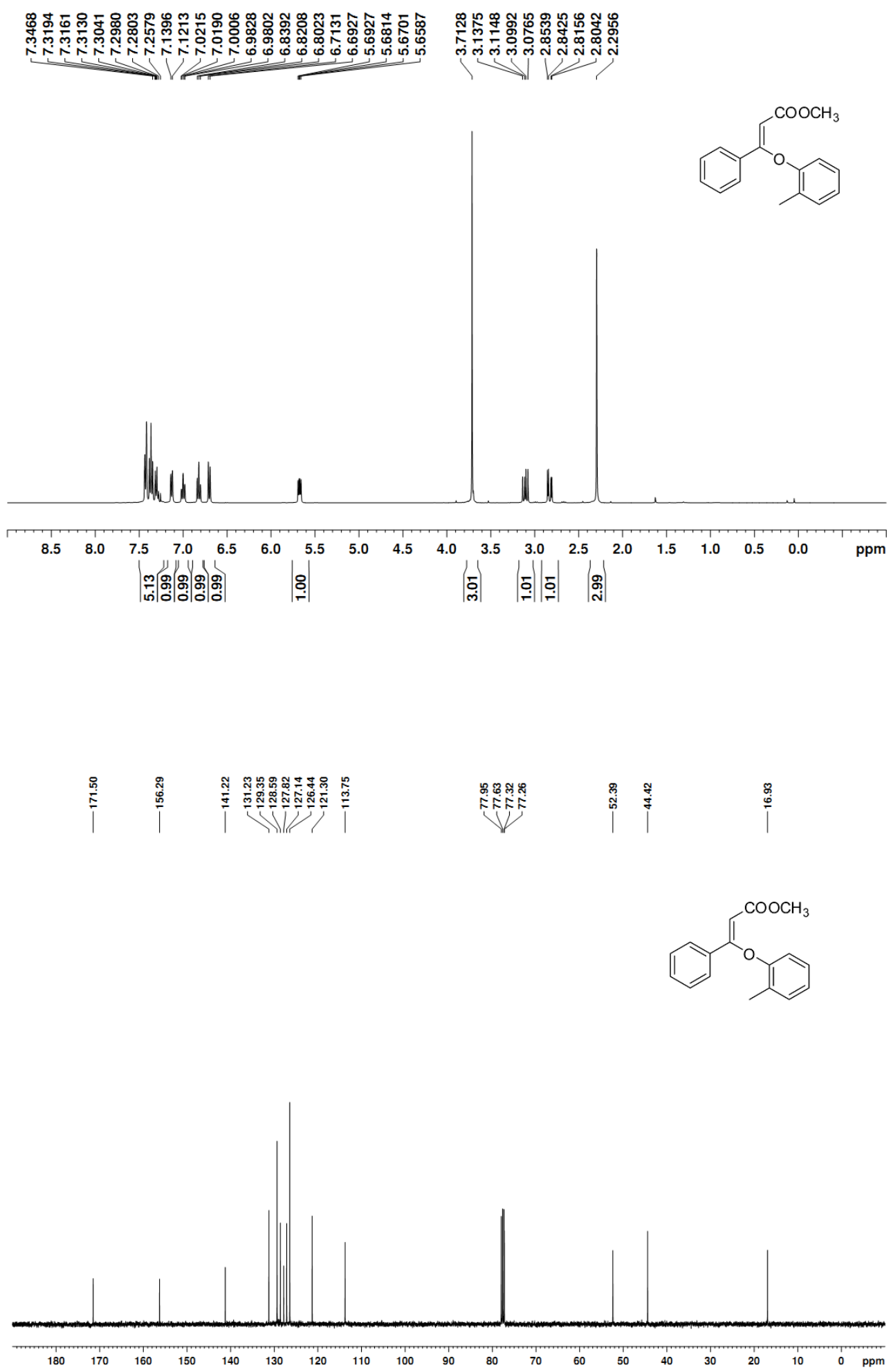
methyl 3-phenyl-3-(p-tolyloxy)propanoate (4d, 4e)

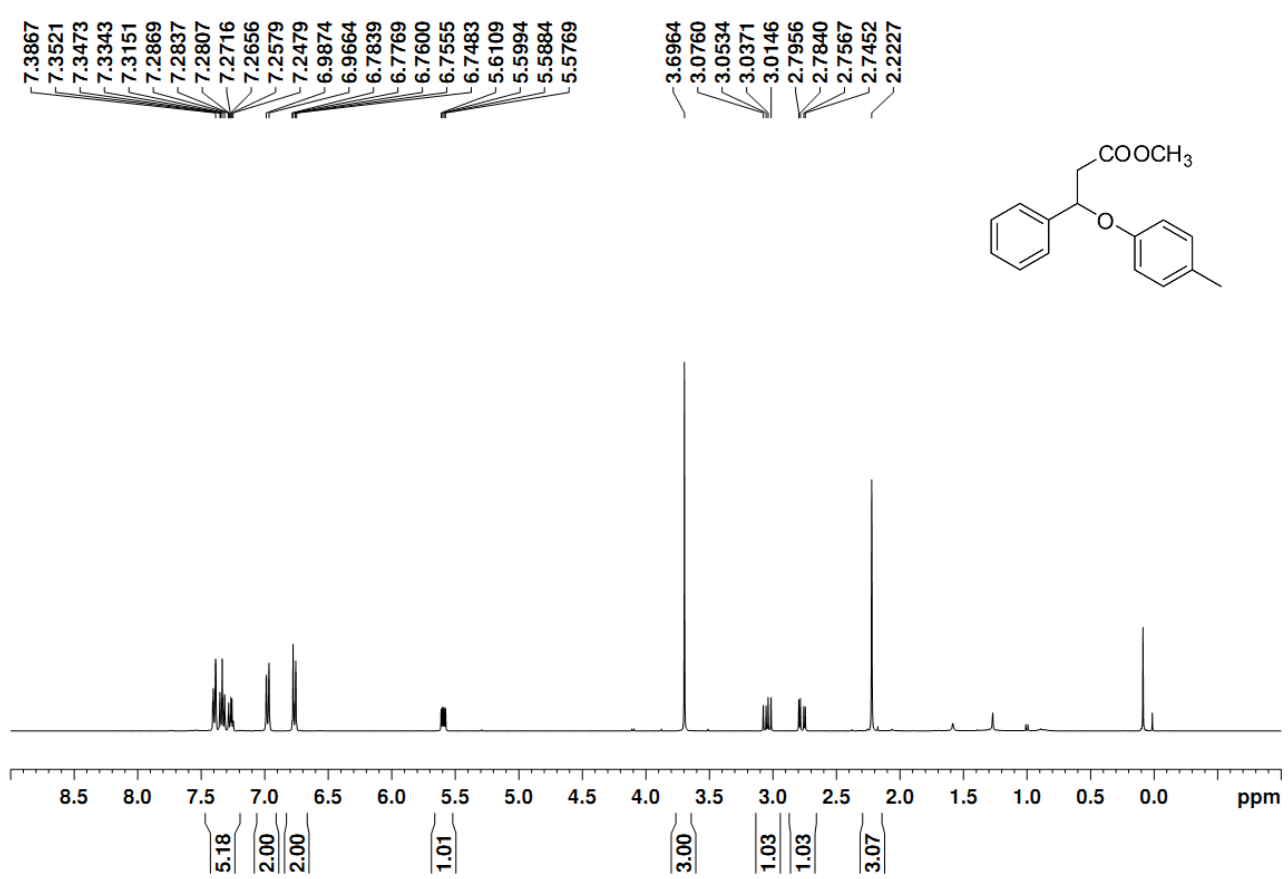

† |

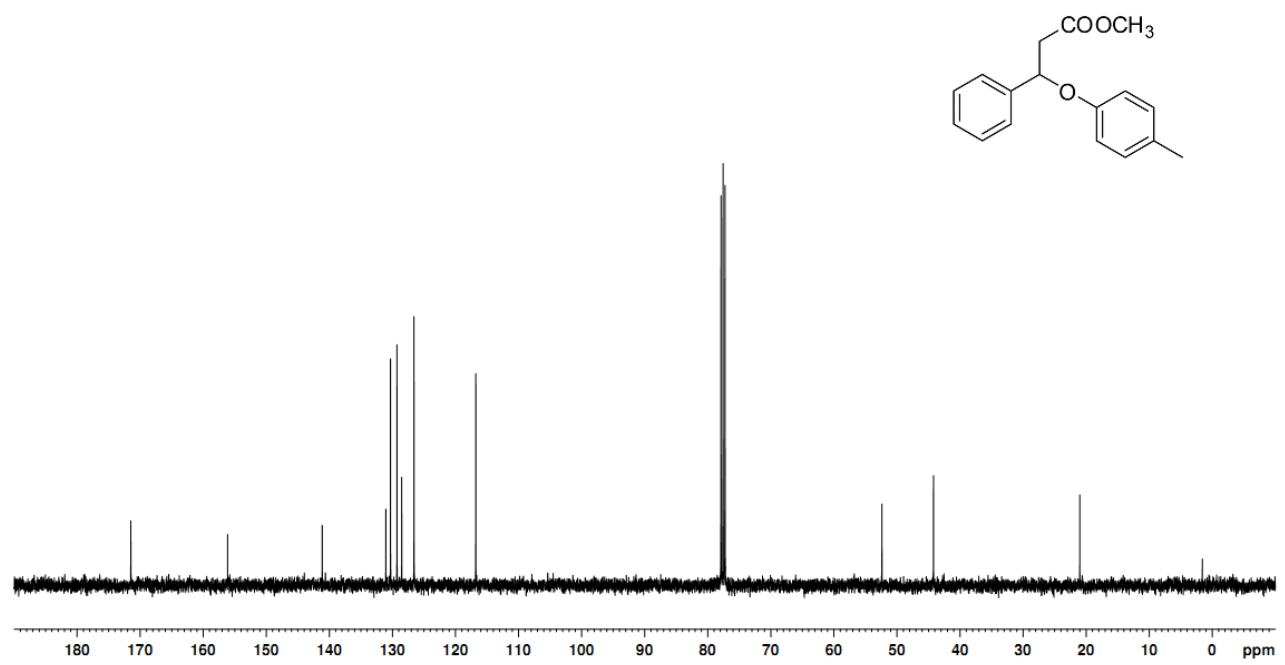


methyl 3-(4-chlorophenoxy)-3-phenylpropanoate (4f)

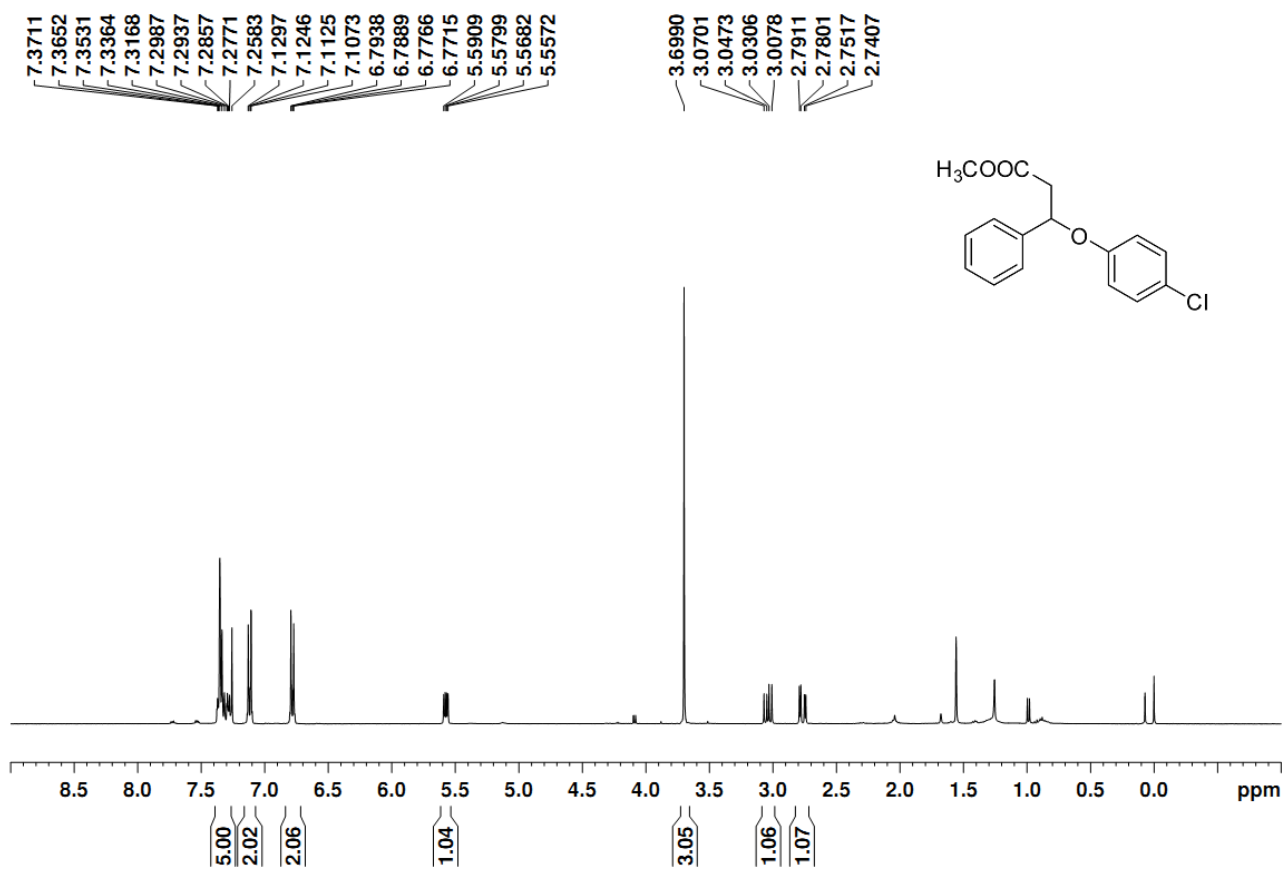

草

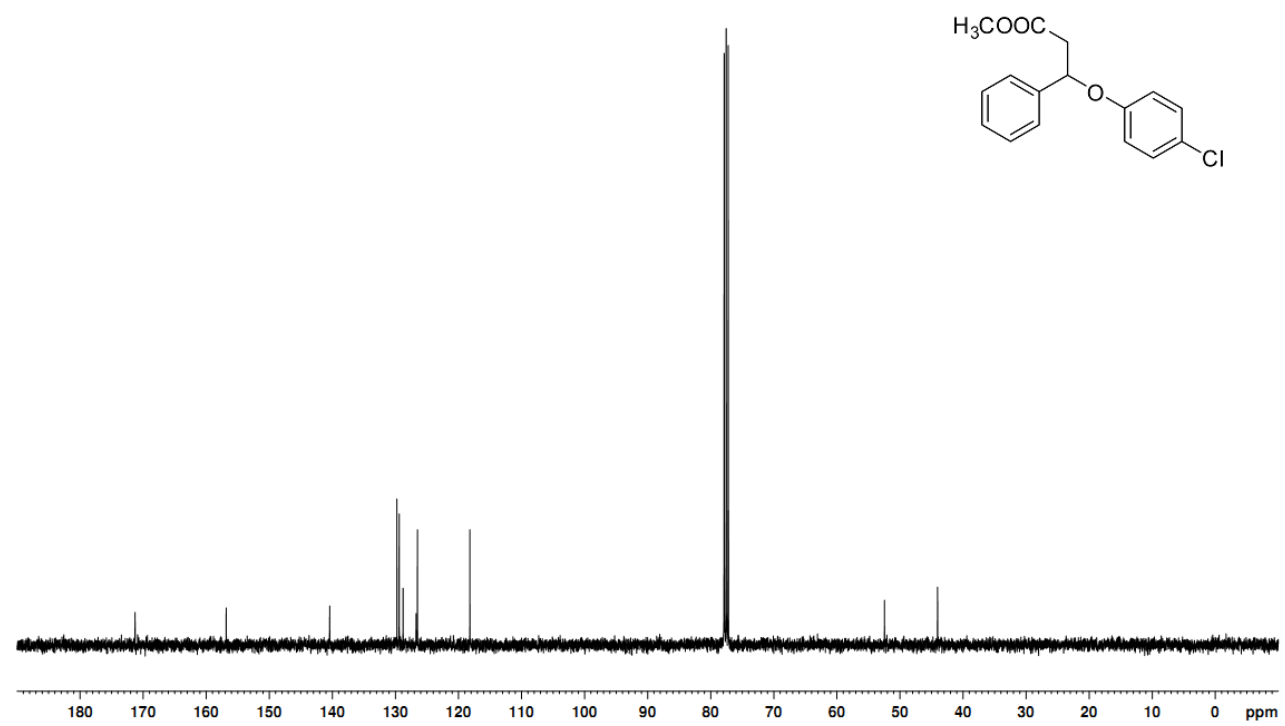


ethyl 3-phenoxybutanoate (4g)

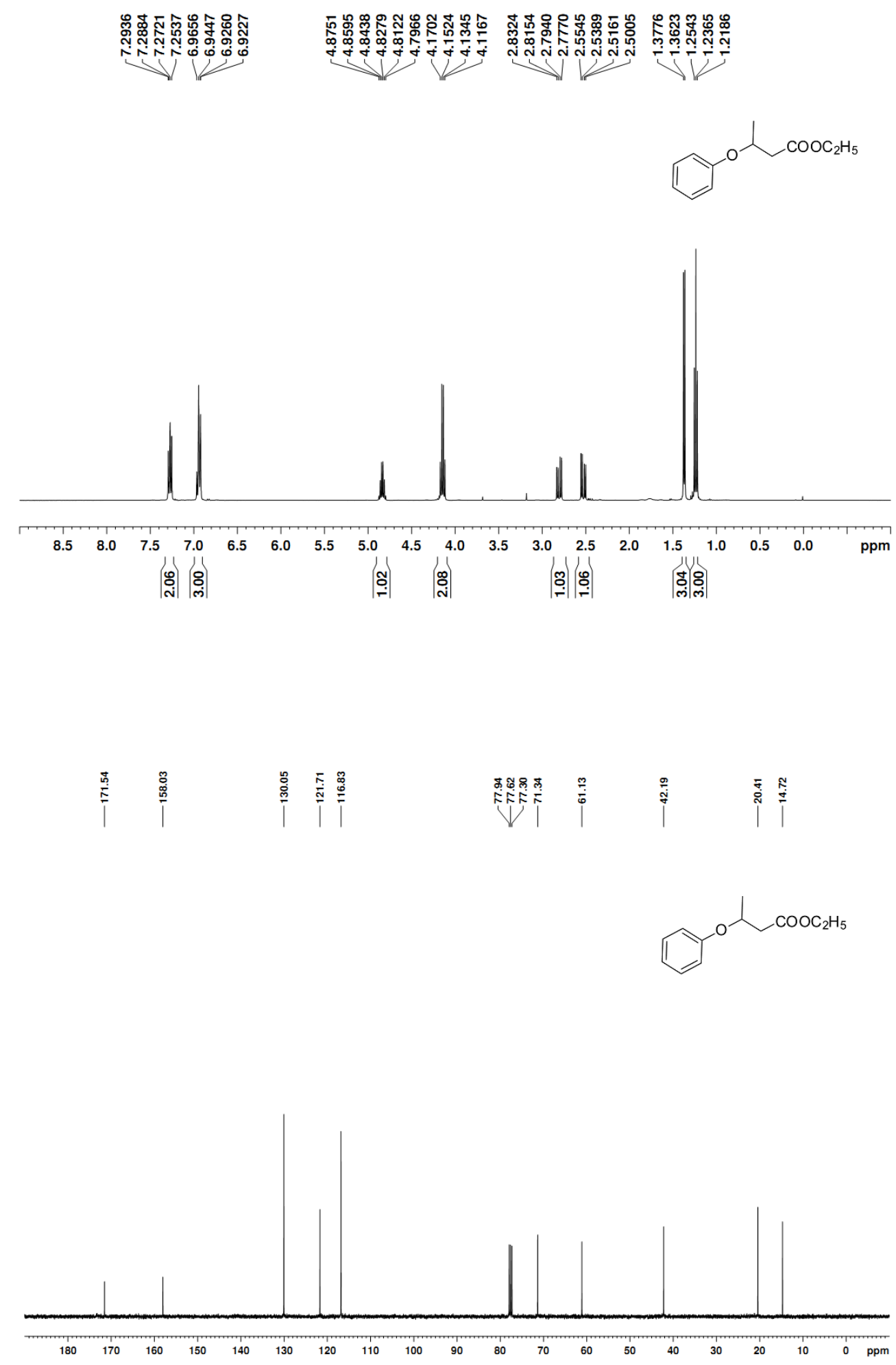



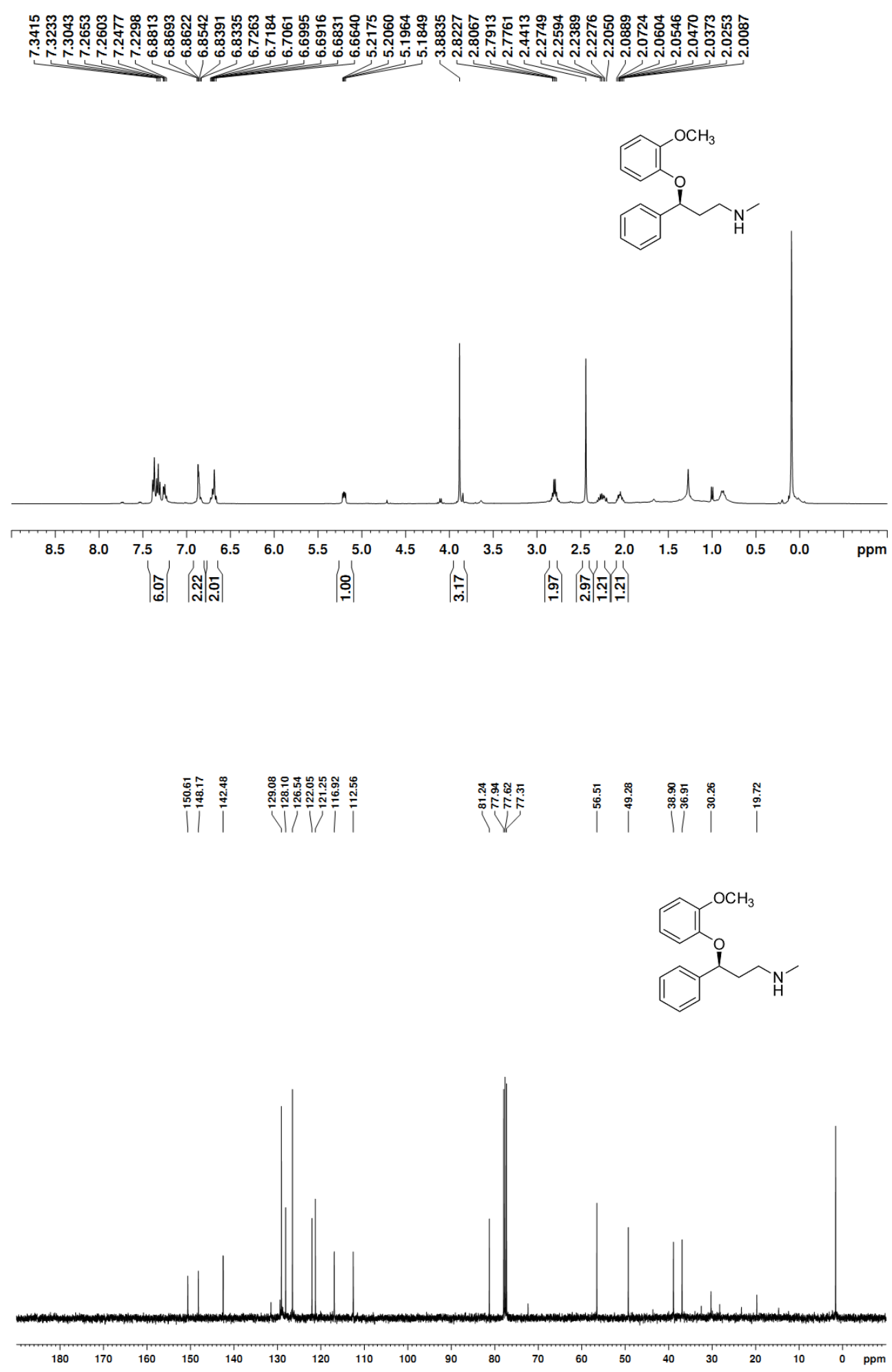


\section{(-)-3-phenoxy-3-phenylpropanenitrile (2a)}

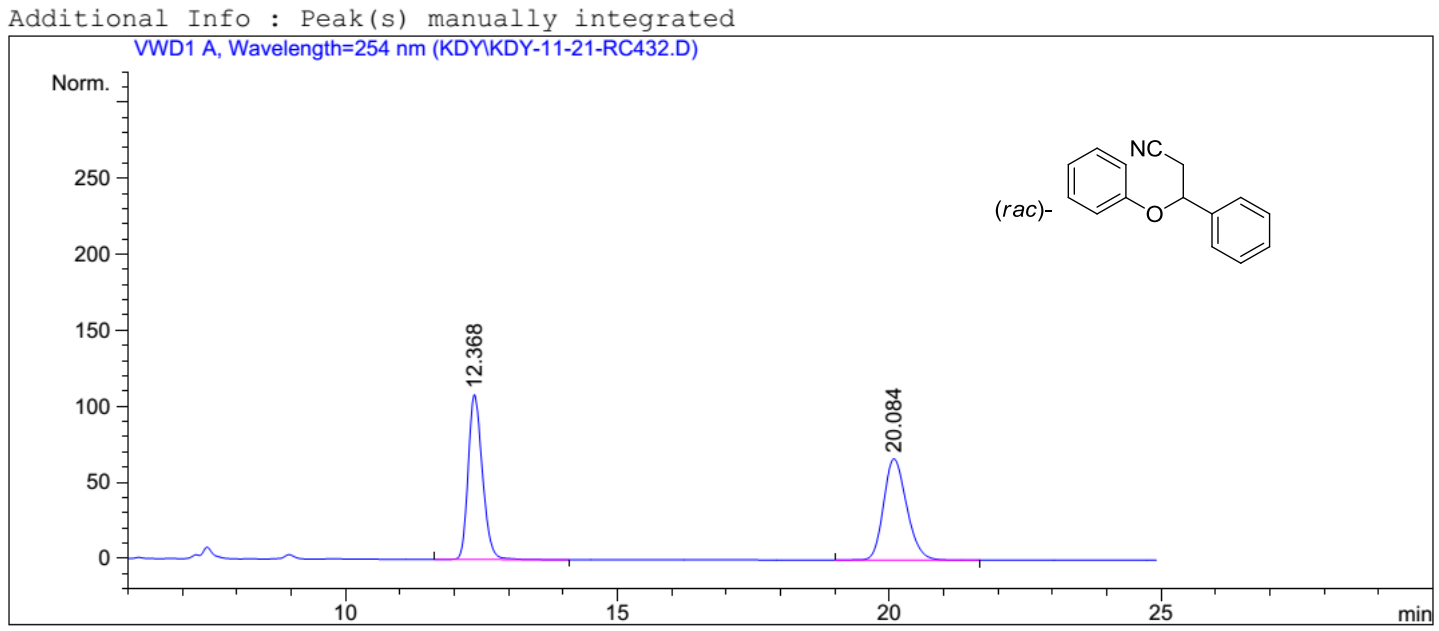

Additional Info : Peak(s) manually integrated

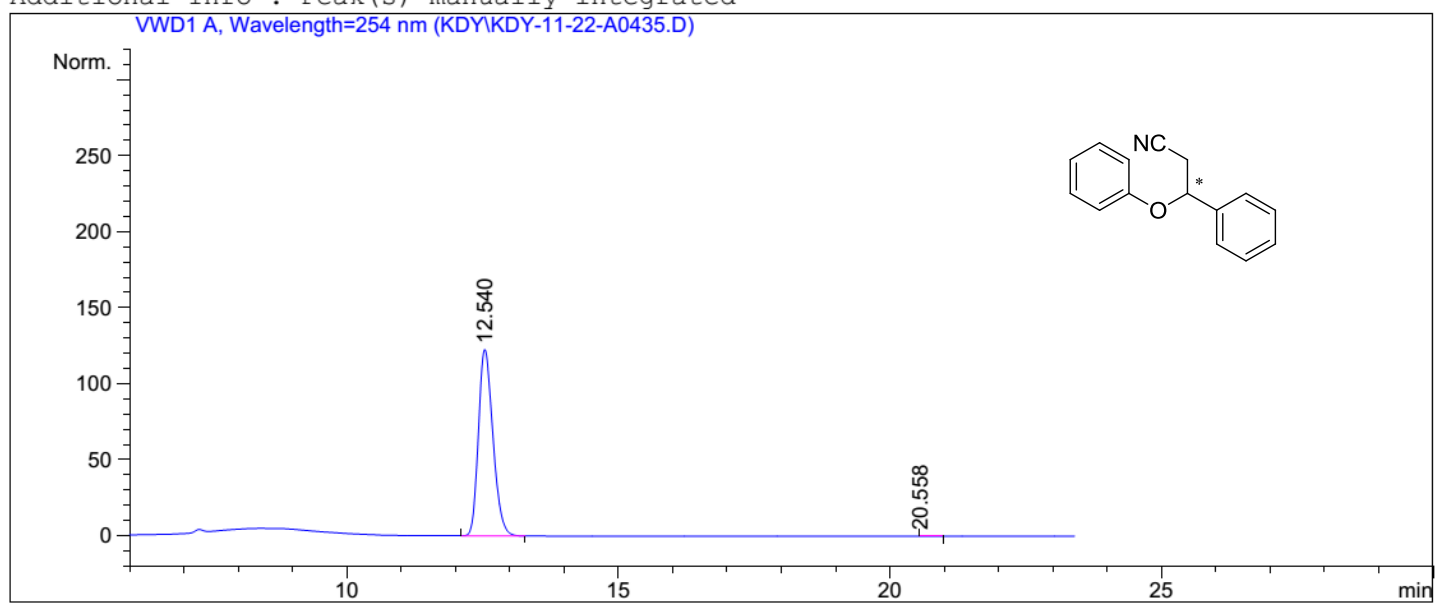

$$
\begin{aligned}
& \text { Peak RetTime Type Width Area Height Area } \\
& \text { \# }[\mathrm{min}] \quad[\mathrm{min}] \quad\left[\mathrm{mAU}{ }^{*} \mathrm{~s}\right] \quad[\mathrm{mAU}] \quad \%
\end{aligned}
$$

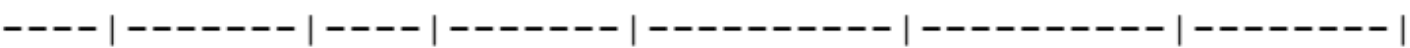

$$
\begin{aligned}
& \begin{array}{lllllll}
1 & 12.540 & \text { VV } & 0.2860 & 2270.51416 & 122.64658 & 99.9697
\end{array} \\
& 220.558 \mathrm{VV} \quad 0.15996 .87970 \mathrm{e}-1 \quad 5.25067 \mathrm{e}-2 \quad 0.0303
\end{aligned}
$$

Totals :

$2271.20213 \quad 122.69909$ 


\section{3-phenyl-3-(o-tolyloxy)propanenitrile (2b)}

Additional Info : Peak(s) manually integrated

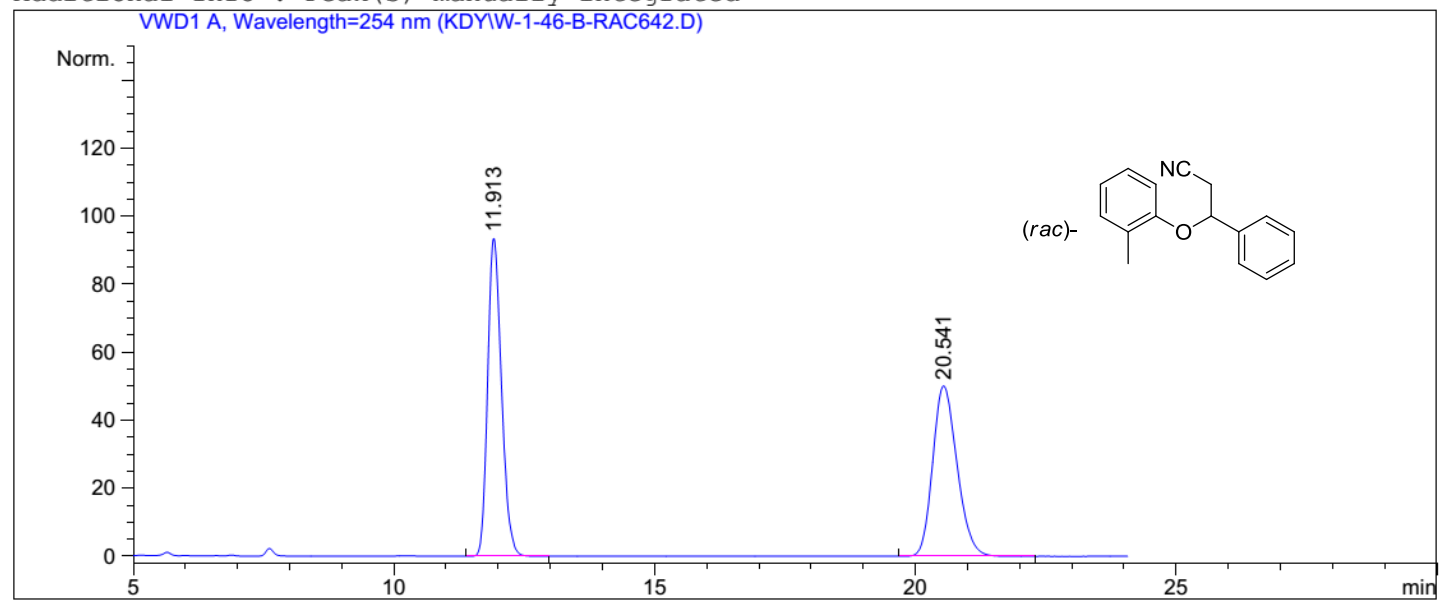

Additional Info : Peak(s) manually integrated

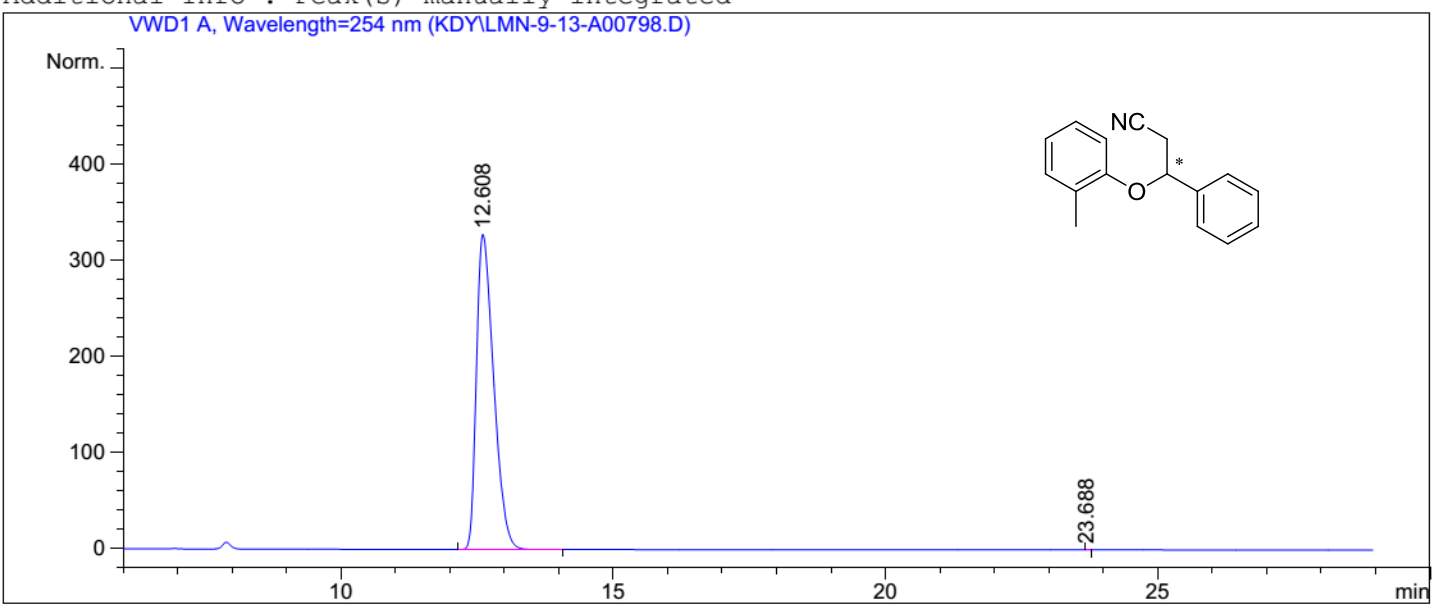

$$
\begin{aligned}
& \text { Peak RetTime Type Width Area Height Area } \\
& \begin{array}{llll}
\# & {[\mathrm{~min}]} & {[\mathrm{min}]} & {\left[\mathrm{mAU}{ }^{*} \mathrm{~s}\right]}
\end{array}
\end{aligned}
$$

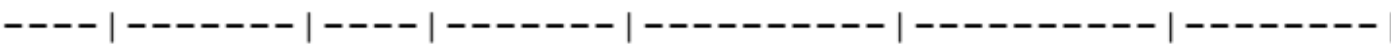

$$
\begin{aligned}
& \begin{array}{lllllll}
1 & 12.608 \text { VV } & 0.3387 & 7192.80957 & 328.24261 & 99.9999
\end{array} \\
& 223.688 \mathrm{VV} \quad 0.04875 .92054 \mathrm{e}-31.61761 \mathrm{e}-3 \quad 8.231 \mathrm{e}-5
\end{aligned}
$$

Totals :

$7192.81549 \quad 328.24423$ 


\section{3-(2-methoxyphenoxy)-3-phenylpropanenitrile (2c)}

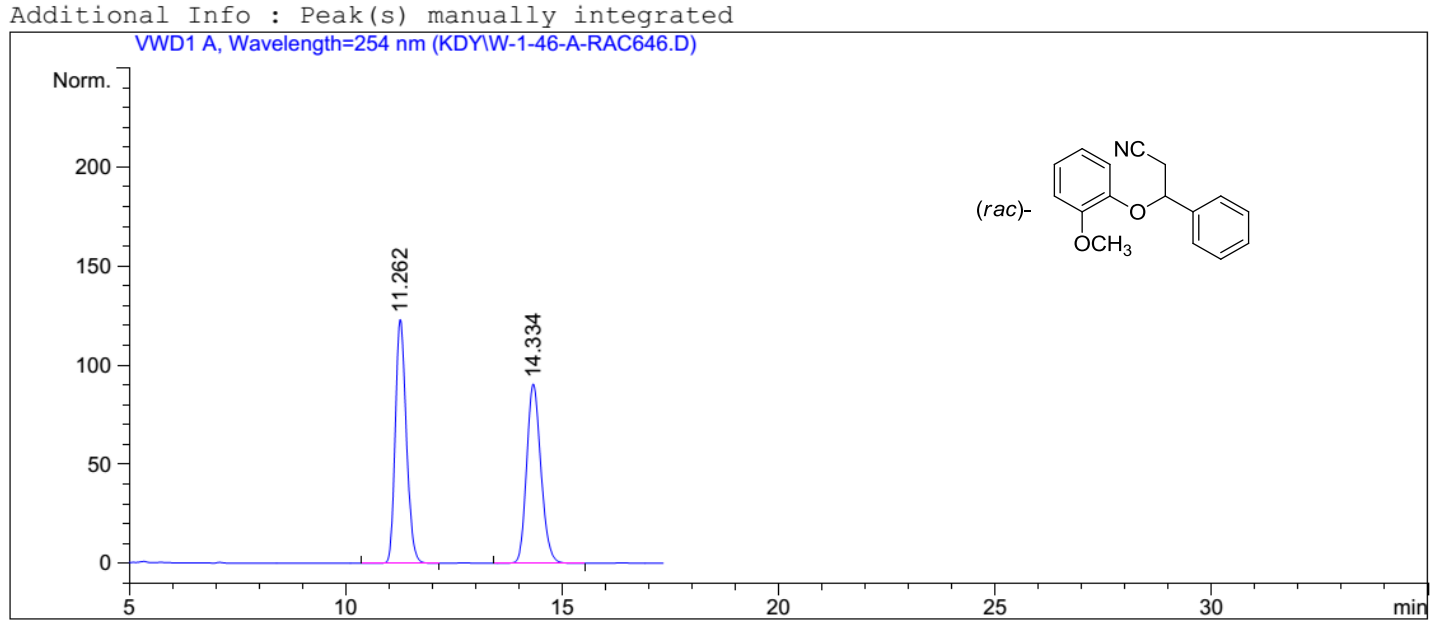

Additional Info : Peak(s) manually integrated

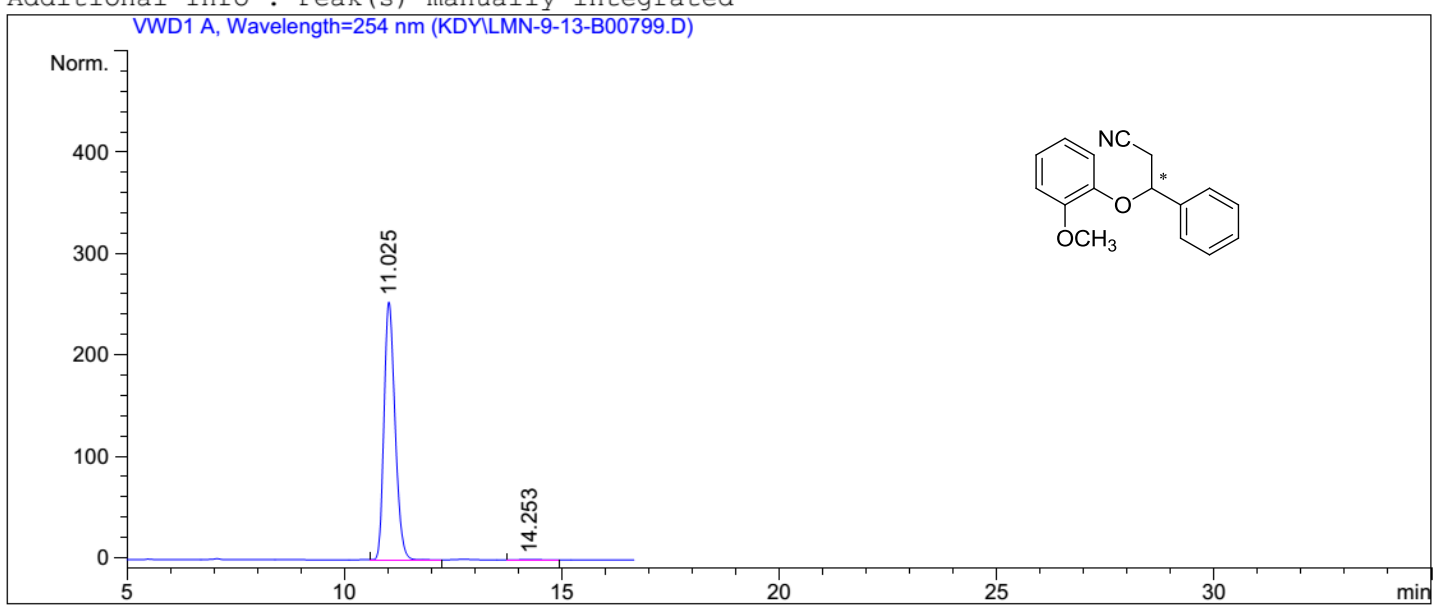

$$
\begin{aligned}
& \text { Peak RetTime Type Width Area Height Area } \\
& \text { \# }[\mathrm{min}] \quad[\mathrm{min}] \quad\left[\mathrm{mAU}^{*} \mathrm{~S}\right] \quad[\mathrm{mAU}] \quad \text { \% }
\end{aligned}
$$

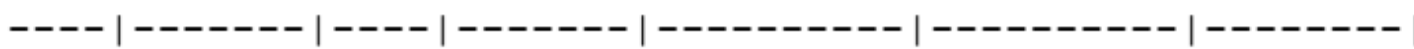

$$
\begin{aligned}
& \begin{array}{lllllll}
1 & 11.025 & \text { VB } & 0.2724 & 4478.13574 & 254.37279 & 99.9082
\end{array} \\
& 214.253 \text { BB } \quad 0.2909 \quad 4.115181 .77891 \mathrm{e}-1 \quad 0.0918
\end{aligned}
$$

Totals :

$4482.25092 \quad 254.55068$ 
(+)-3-(2-chlorophenoxy)-3-phenylpropanenitrile (2d)
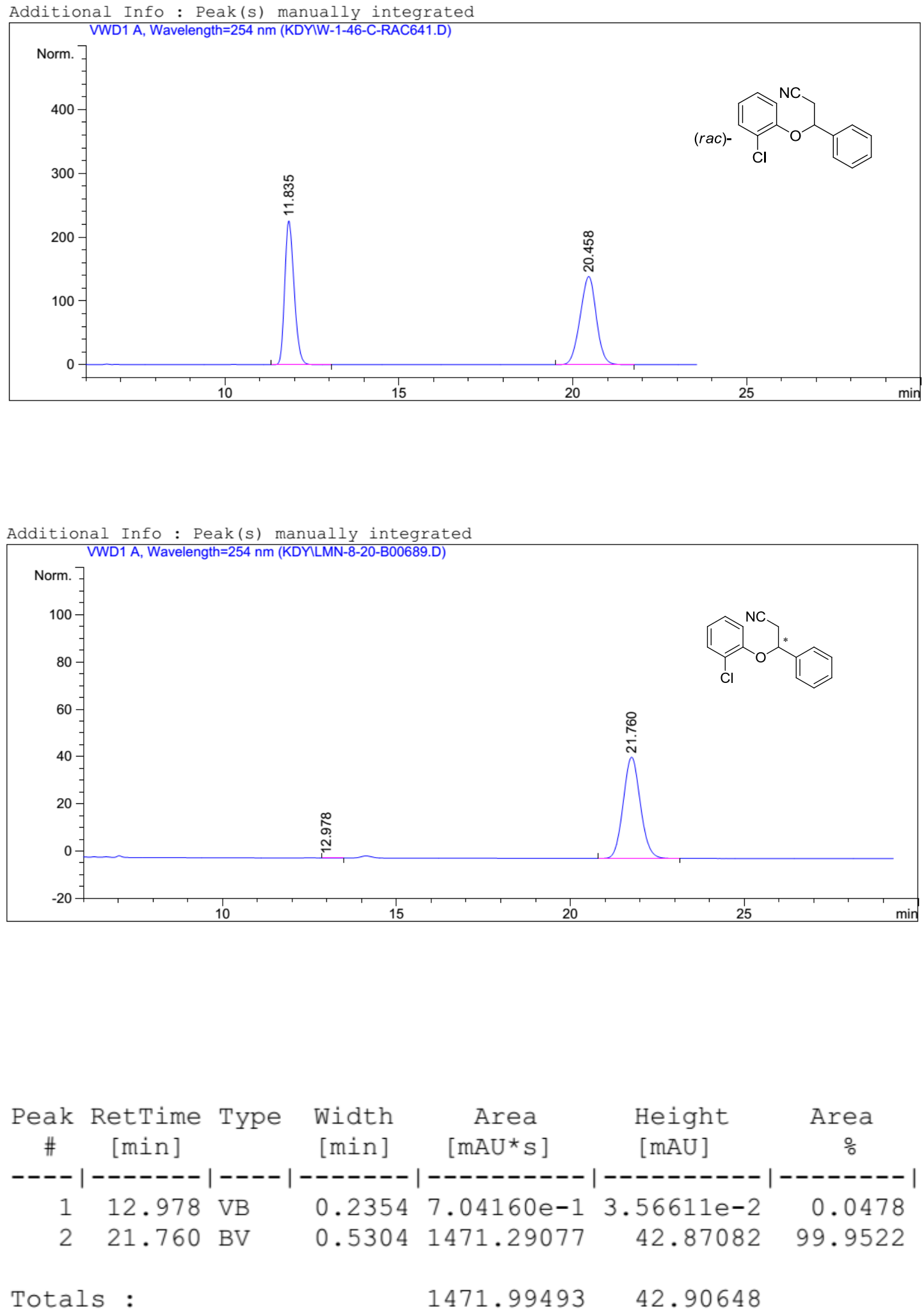


\section{3-(3-chlorophenoxy)-3-phenylpropanenitrile (2e)}

Additional Info: Peak(s) manually integrated

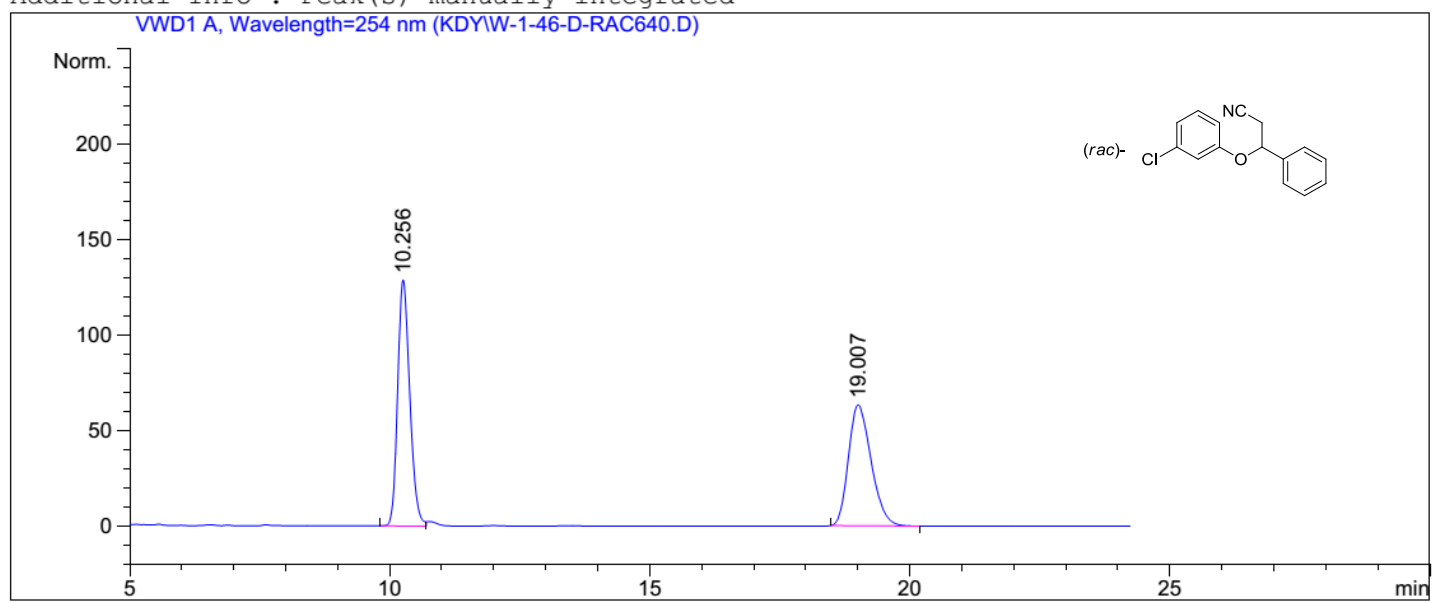

Additional Info : Peak(s) manually integrated

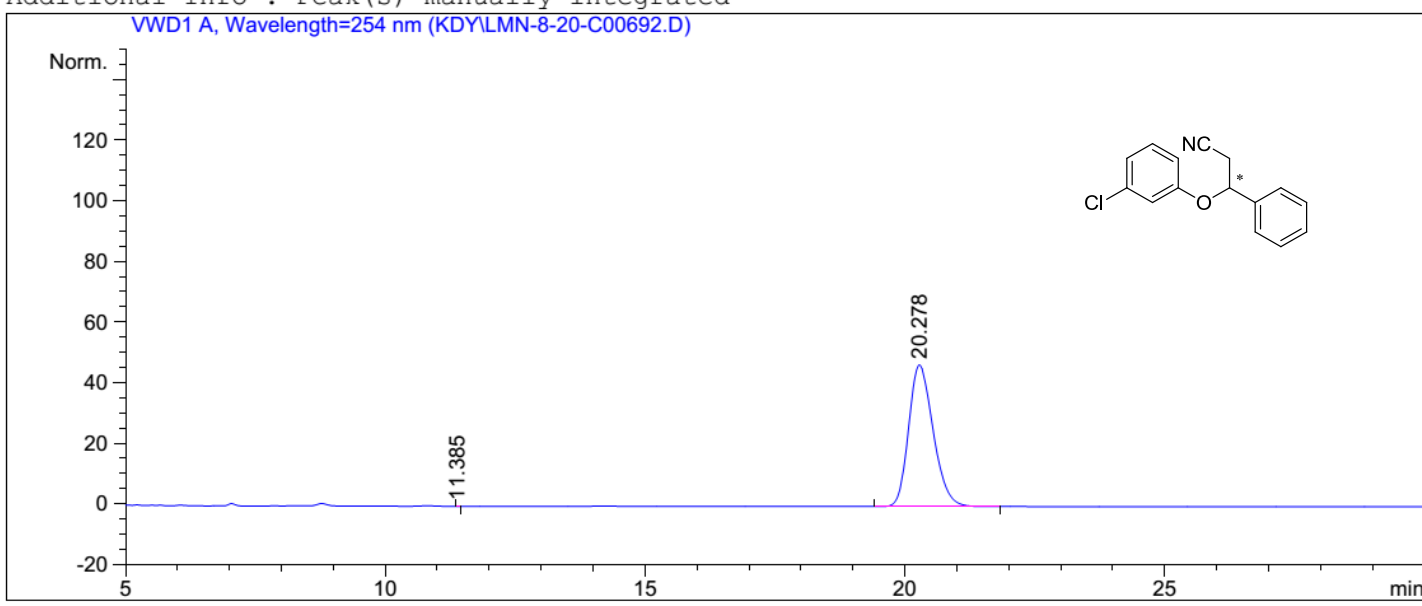

$$
\begin{aligned}
& \text { Peak RetTime Type Width Area Height Area } \\
& \text { \# }[\mathrm{min}] \quad[\mathrm{min}] \quad\left[\mathrm{mAU}{ }^{*} \mathrm{~s}\right] \quad[\mathrm{mAU}] \quad \text { \% }
\end{aligned}
$$

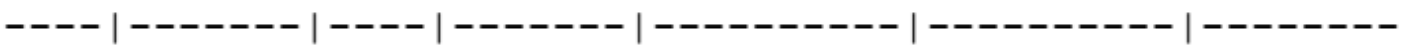

$$
\begin{aligned}
& 111.385 \text { BV } 0.04167 .98794 \mathrm{e}-3 \quad 2.48162 \mathrm{e}-3 \quad 5.219 \mathrm{e}-4 \\
& 2 \quad 20.278 \text { VV } \quad 0.5086 \quad 1530.41772 \quad 46.67207 \quad 99.9995
\end{aligned}
$$

Totals : $\quad 1530.42571 \quad 46.67455$ 


\section{(-)-3-phenyl-3-(p-tolyloxy)propanenitrile (2f)}

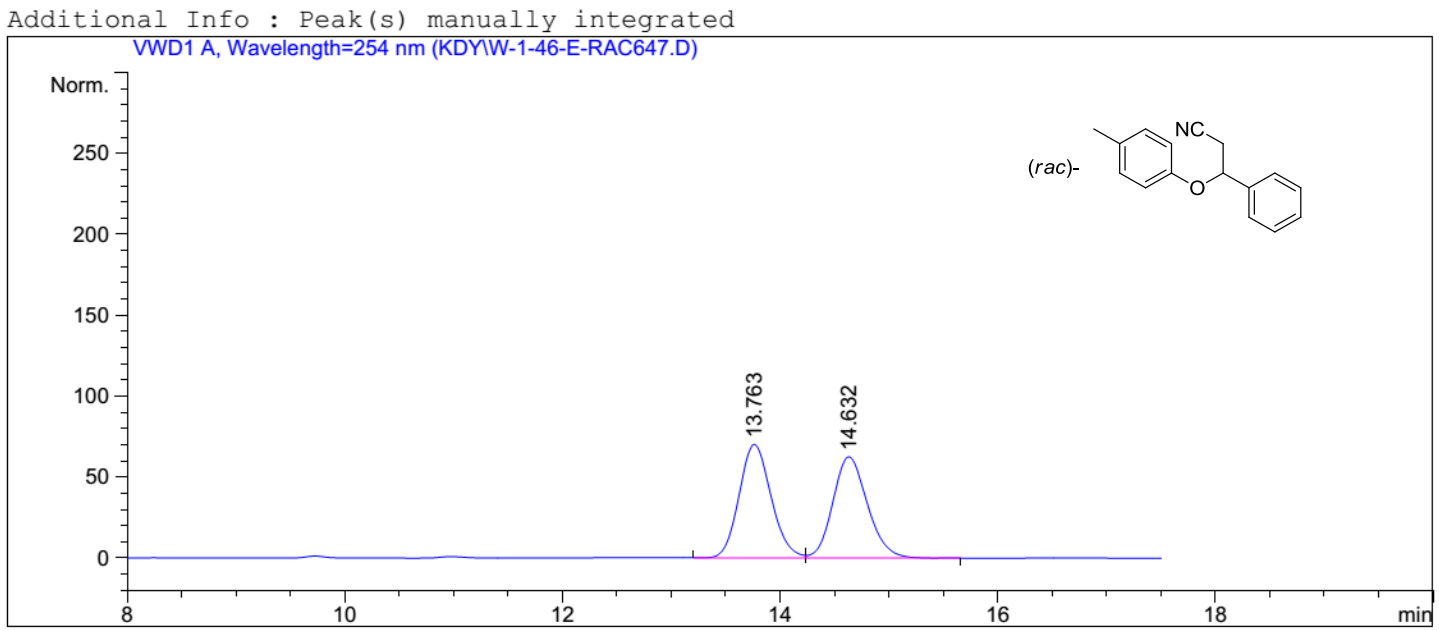

Additional Info : Peak(s) manually integrated
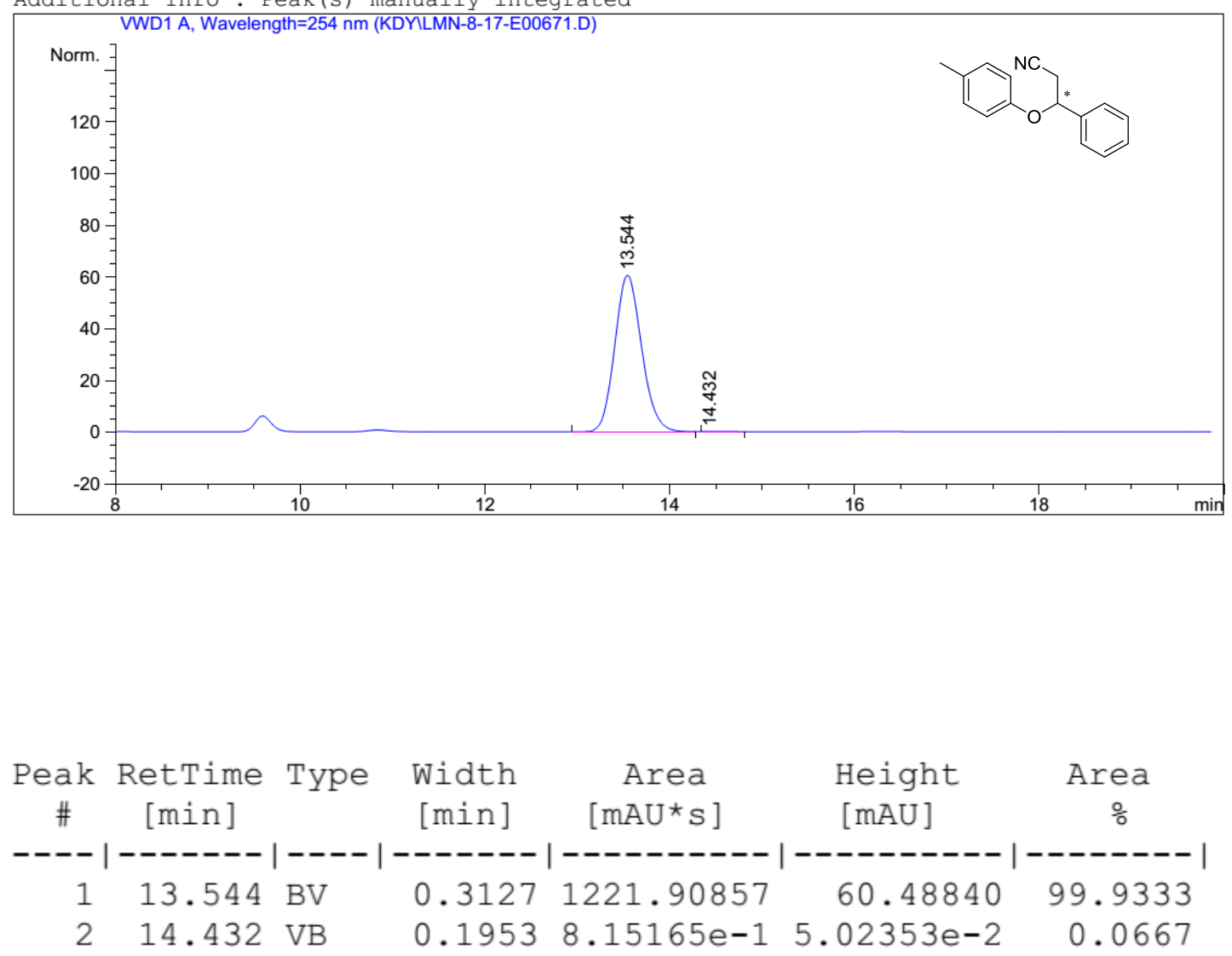

Totals :

$1222.72373 \quad 60.53863$ 


\section{3-(4-chlorophenoxy)-3-phenylpropanenitrile (2g)}

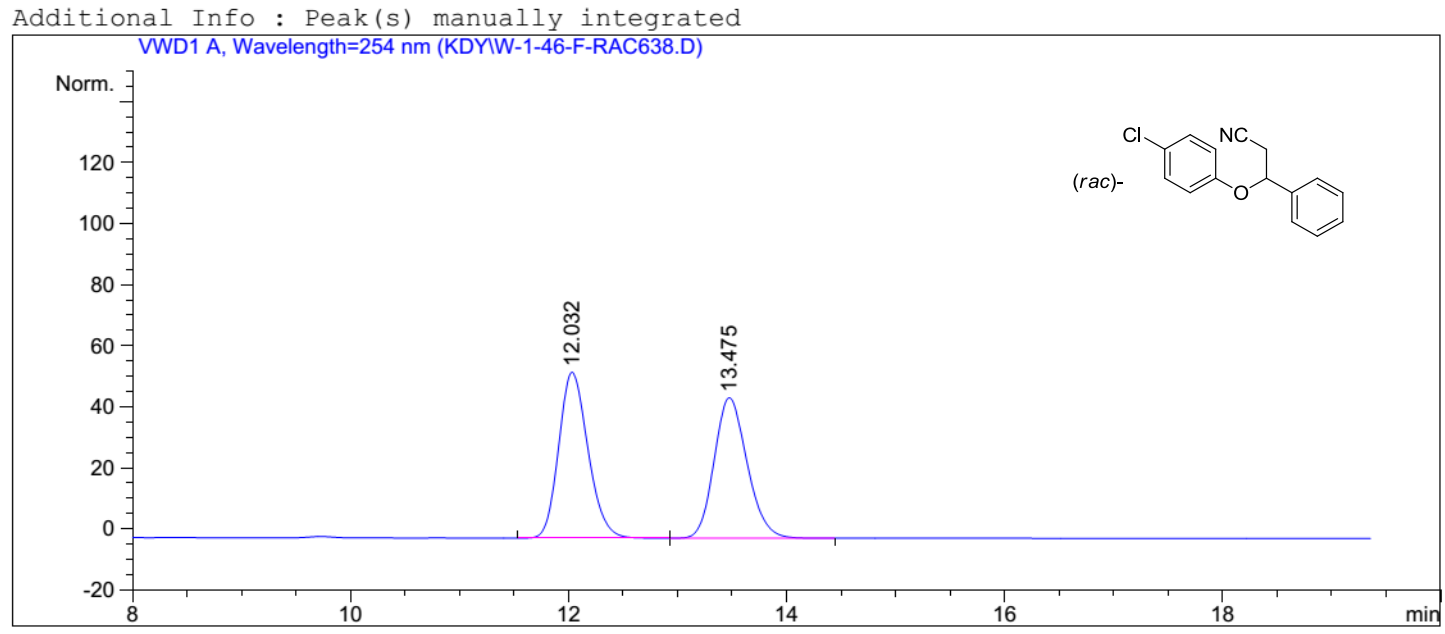

Additional Info : Peak(s) manually integrated

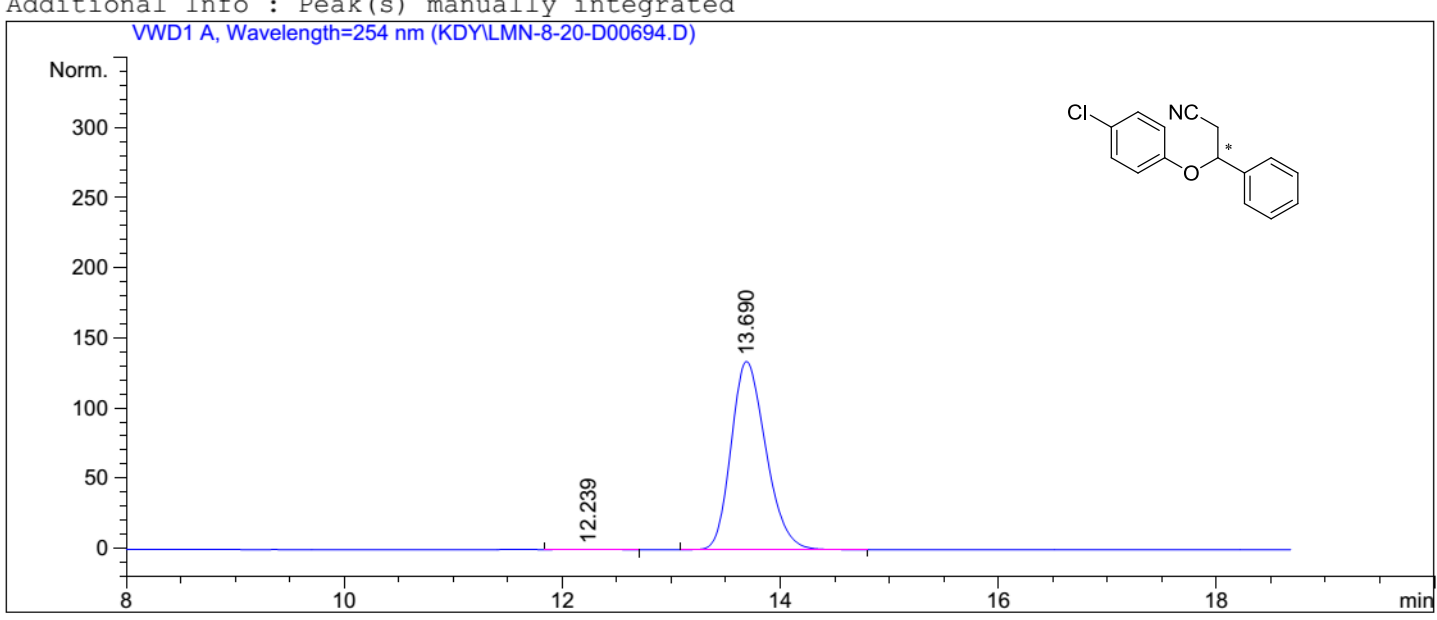

\begin{tabular}{|c|c|c|c|c|c|c|}
\hline $\begin{array}{c}\text { Peak } \\
\quad \#\end{array}$ & $\begin{array}{c}\text { RetTime } \\
\text { [min] }\end{array}$ & Type & $\begin{array}{l}\text { Width } \\
\text { [min] }\end{array}$ & $\begin{array}{c}\text { Area } \\
{\left[\mathrm{mAU}^{\star} \mathrm{s}\right]}\end{array}$ & $\begin{array}{l}\text { Height } \\
\text { [mAU] }\end{array}$ & $\begin{array}{c}\text { Area } \\
\frac{\circ}{0}\end{array}$ \\
\hline-- & ------- & & ------- & | ---------- & |--------- & -------- \\
\hline 1 & 12.239 & VV & 0.2646 & 4.51453 & $2.26463 e-1$ & 0.1521 \\
\hline 2 & 13.690 & BV & 0.3425 & 2962.77441 & 134.24980 & 99.8479 \\
\hline cal & & & & 2967.28894 & 7626 & \\
\hline
\end{tabular}




\section{3-phenyl-3-(4-(trifluoromethyl)phenoxy)propanenitrile (2h)}

Additional Info : Peak(s) manually integrated

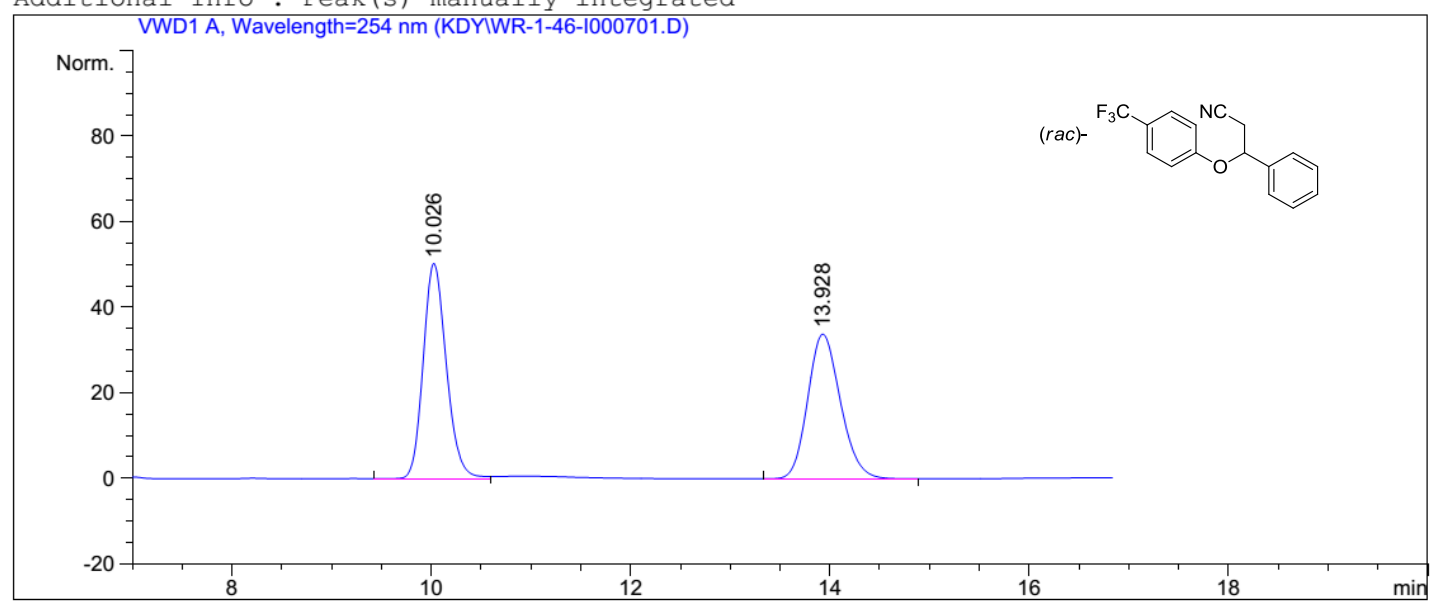

Additional Info : Peak(s) manually integrated

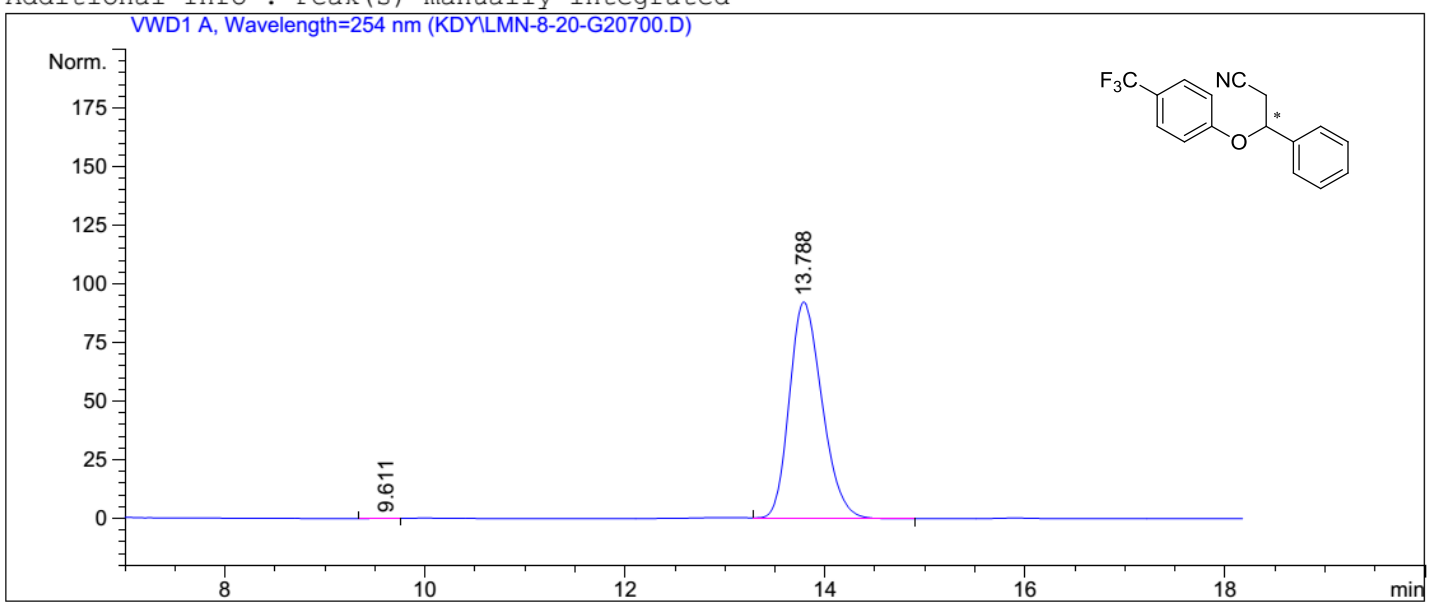

$$
\begin{aligned}
& \text { Peak RetTime Type Width Area Height Area }
\end{aligned}
$$

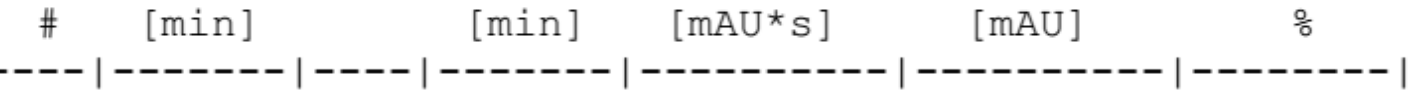

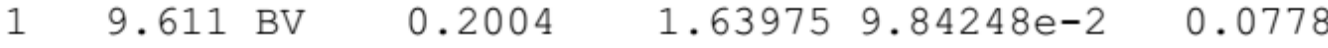

$$
\begin{aligned}
& \begin{array}{lllllll}
2 & 13.788 & \text { VV } & 0.3518 & 2106.17651 & 92.43792 & 99.9222
\end{array}
\end{aligned}
$$

Totals : $\quad 2107.81626 \quad 92.53634$ 


\section{3-(naphthalen-1-yloxy)-3-phenylpropanenitrile (2i)}

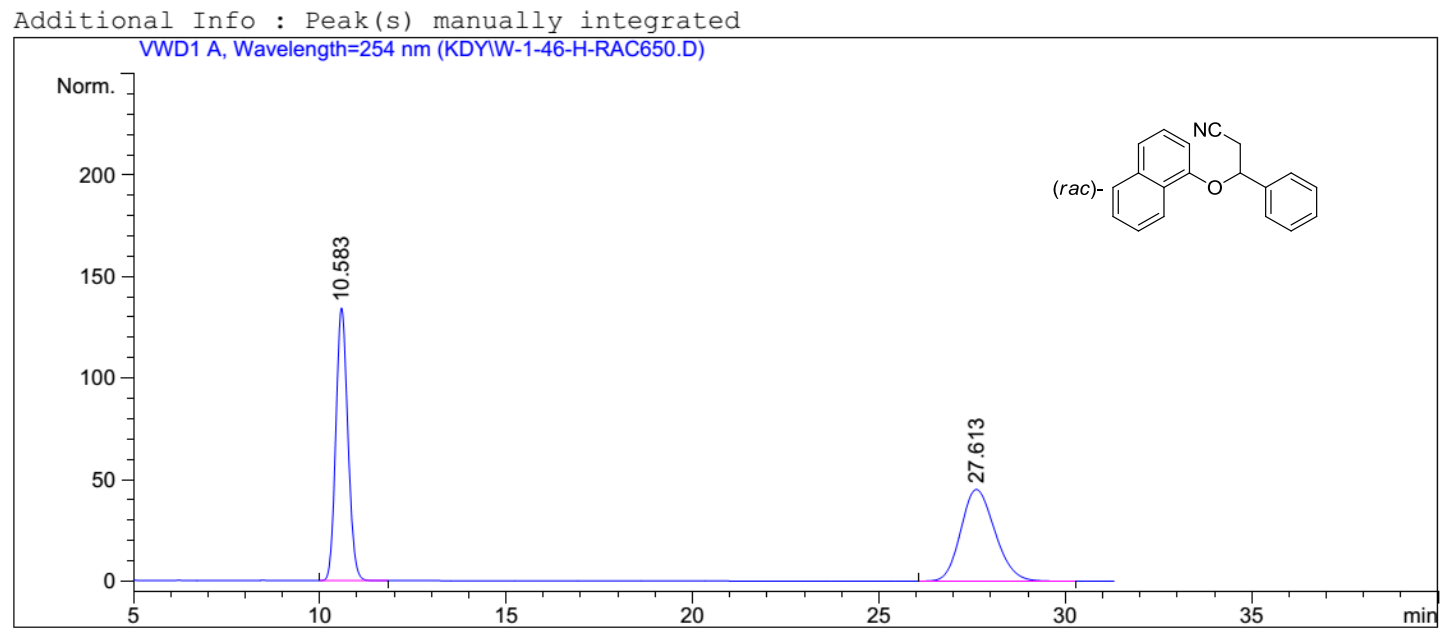

Additional Info : Peak(s) manually integrated

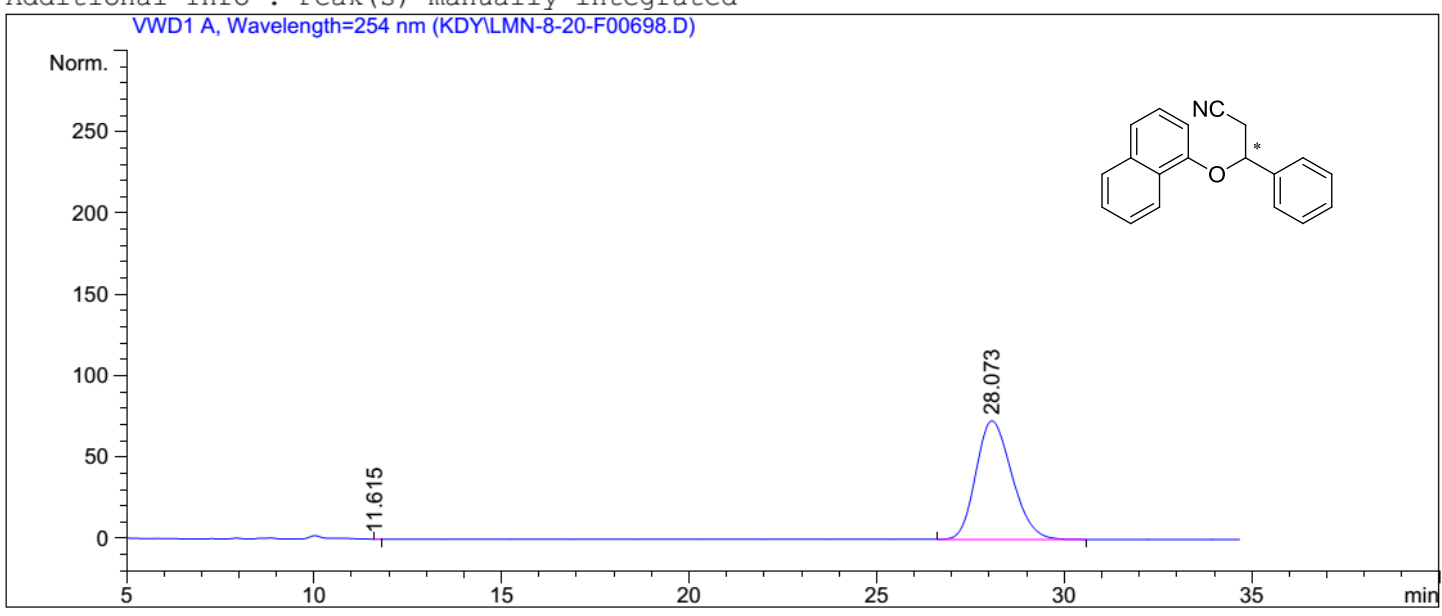

$$
\begin{aligned}
& \text { Peak RetTime Type Width Area Height Area } \\
& \text { \# }[\mathrm{min}] \quad[\mathrm{min}] \quad[\mathrm{mAU} * \mathrm{~S}] \quad[\mathrm{mAU}] \quad \%
\end{aligned}
$$

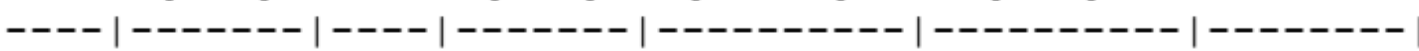

$$
\begin{aligned}
& 1 \quad 11.615 \text { BB } 0.15161 .40935 \mathrm{e}-21.12931 \mathrm{e}-3 \quad 2.894 \mathrm{e}-4
\end{aligned}
$$

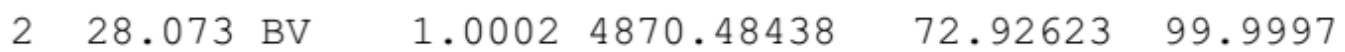

$$
\begin{aligned}
& \text { Totals : } \\
& 4870.49847 \quad 72.92736
\end{aligned}
$$




\section{3-(naphthalen-2-yloxy)-3-phenylpropanenitrile (2j)}

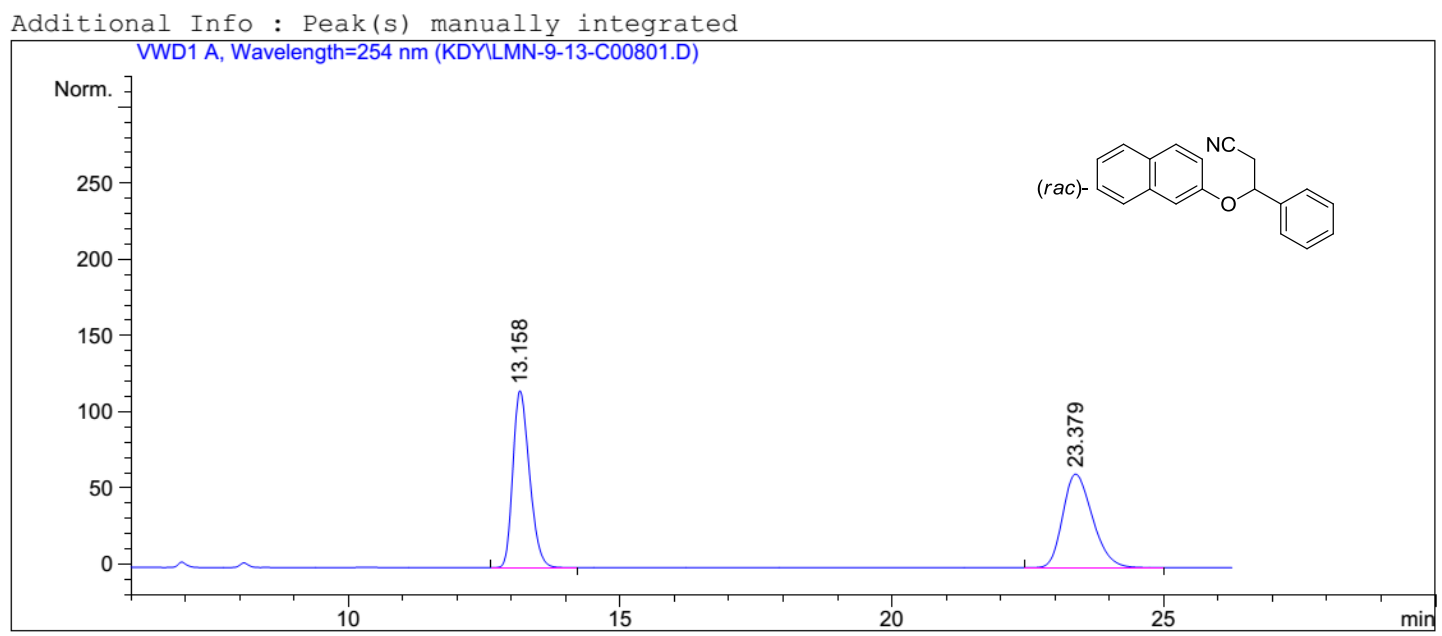

Additional Info : Peak(s) manually integrated
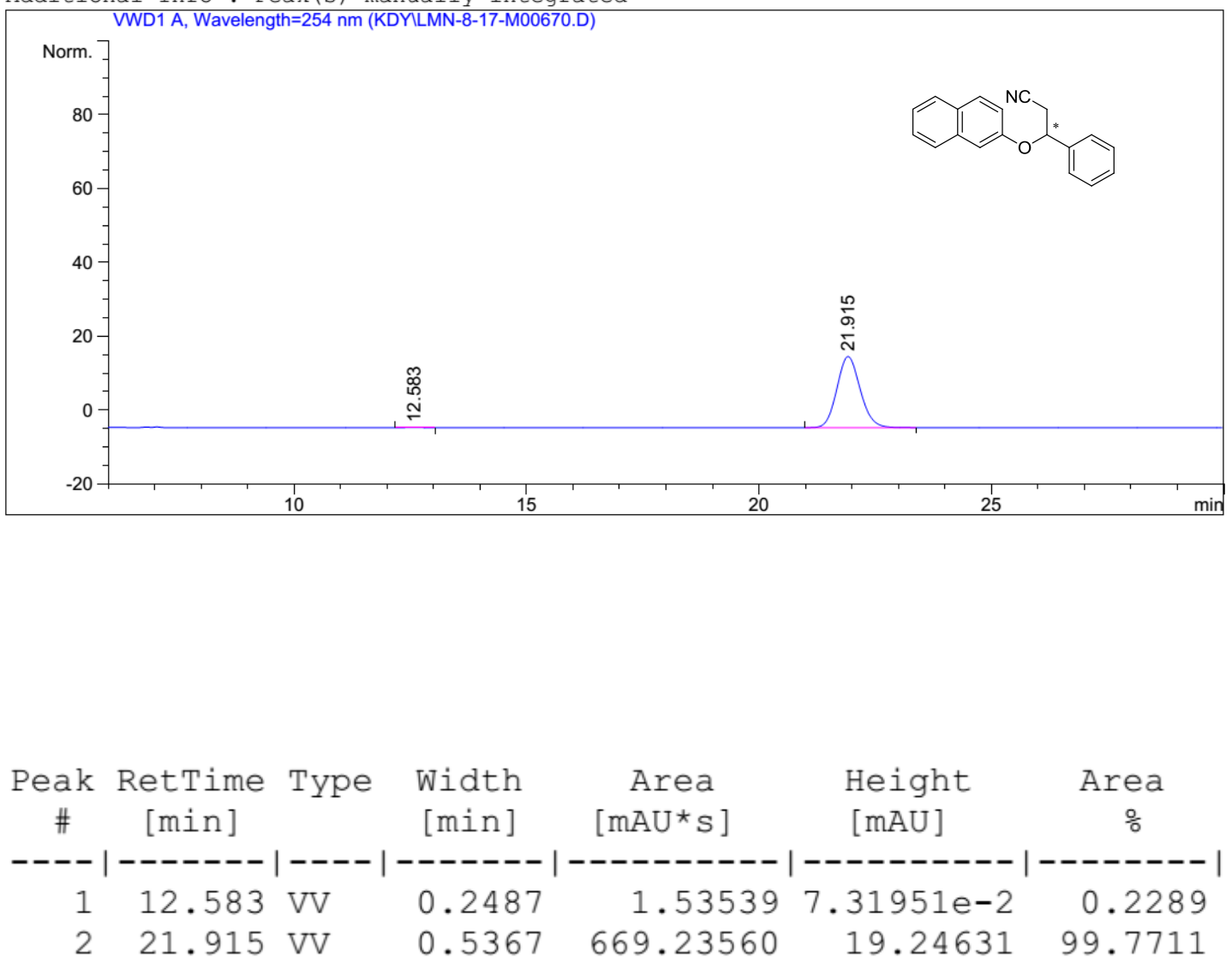
Totals :
$670.77098 \quad 19.31950$ 
(+)-3-phenoxy-3-phenylpropanenitrile (2k)

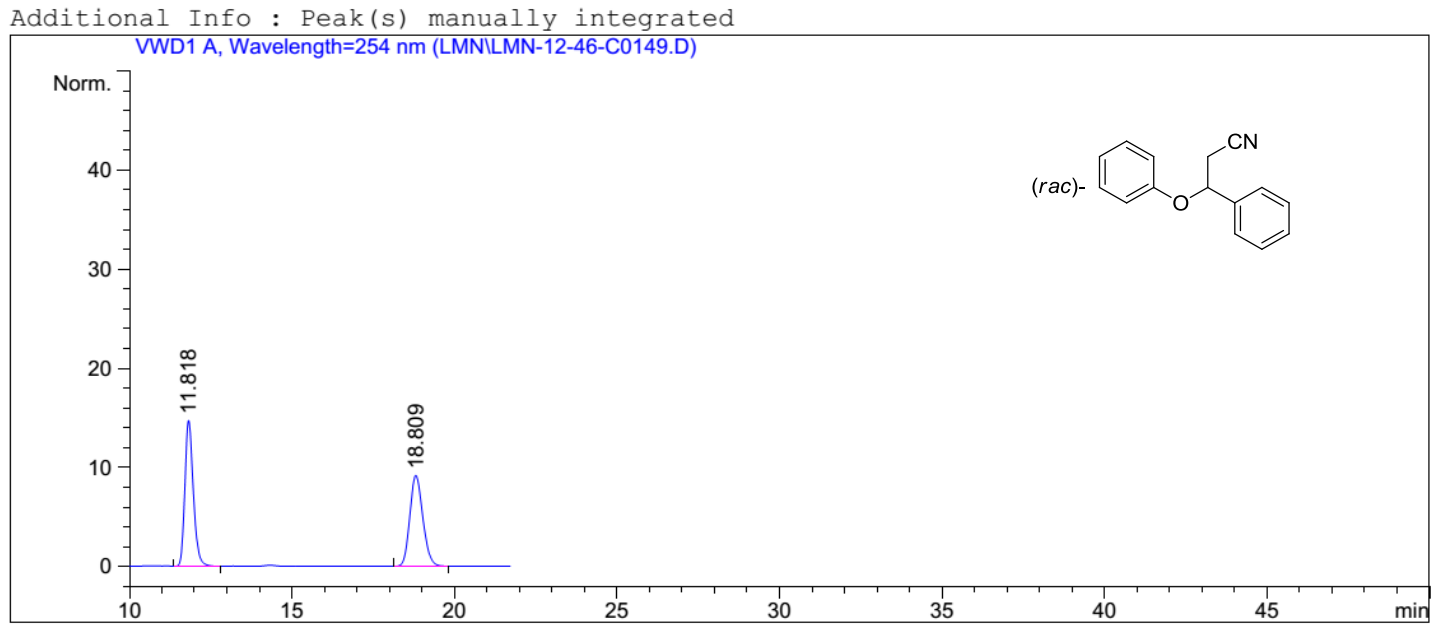

Additional Info : Peak(s) manually integrated

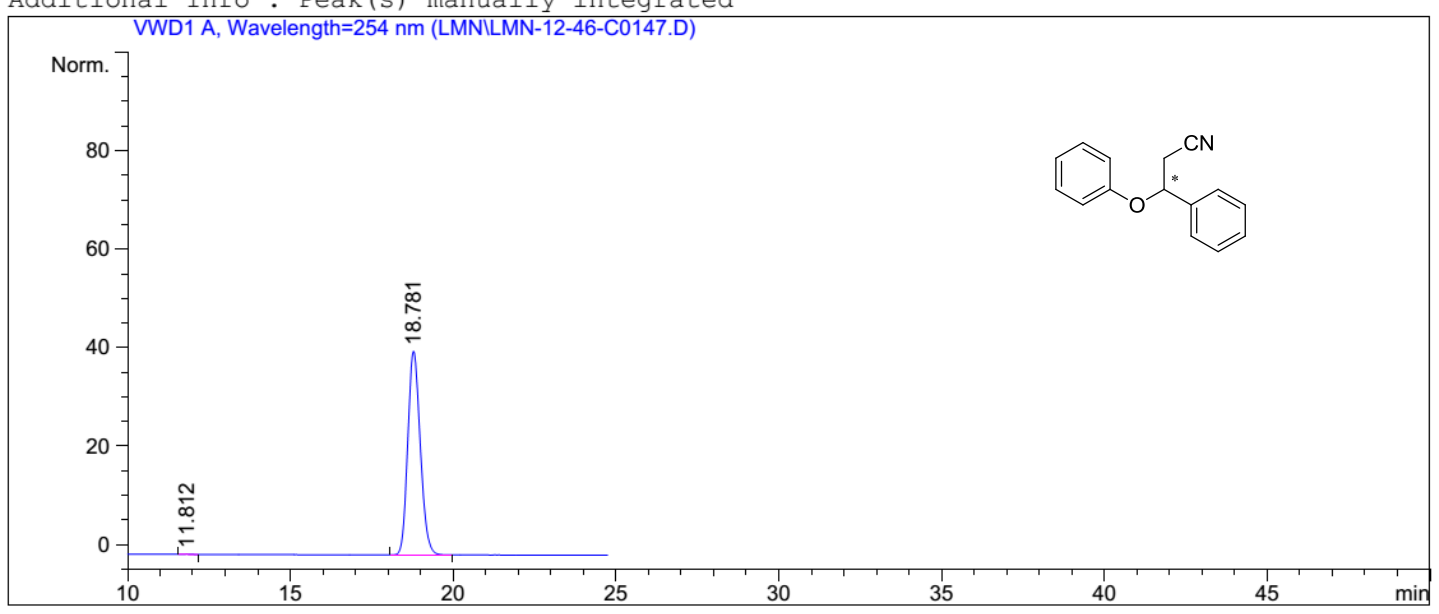

$$
\begin{aligned}
& \text { Peak RetTime Type Width Area Height Area } \\
& \begin{array}{llll}
\# & {[\mathrm{~min}]} & {[\mathrm{min}]} & {[\mathrm{mAU} * \mathrm{~s}]}
\end{array}
\end{aligned}
$$

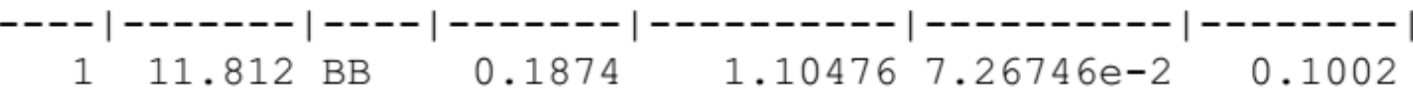

$$
\begin{aligned}
& \begin{array}{lllllll}
2 & 18.781 & \text { BB } & 0.4114 & 1101.36145 & 41.39447 & 99.8998
\end{array} \\
& \text { Totals : } \quad 1102.46621 \quad 41.46715
\end{aligned}
$$


(-)-3-(2-chlorophenoxy)-3-phenylpropanenitrile (2l)

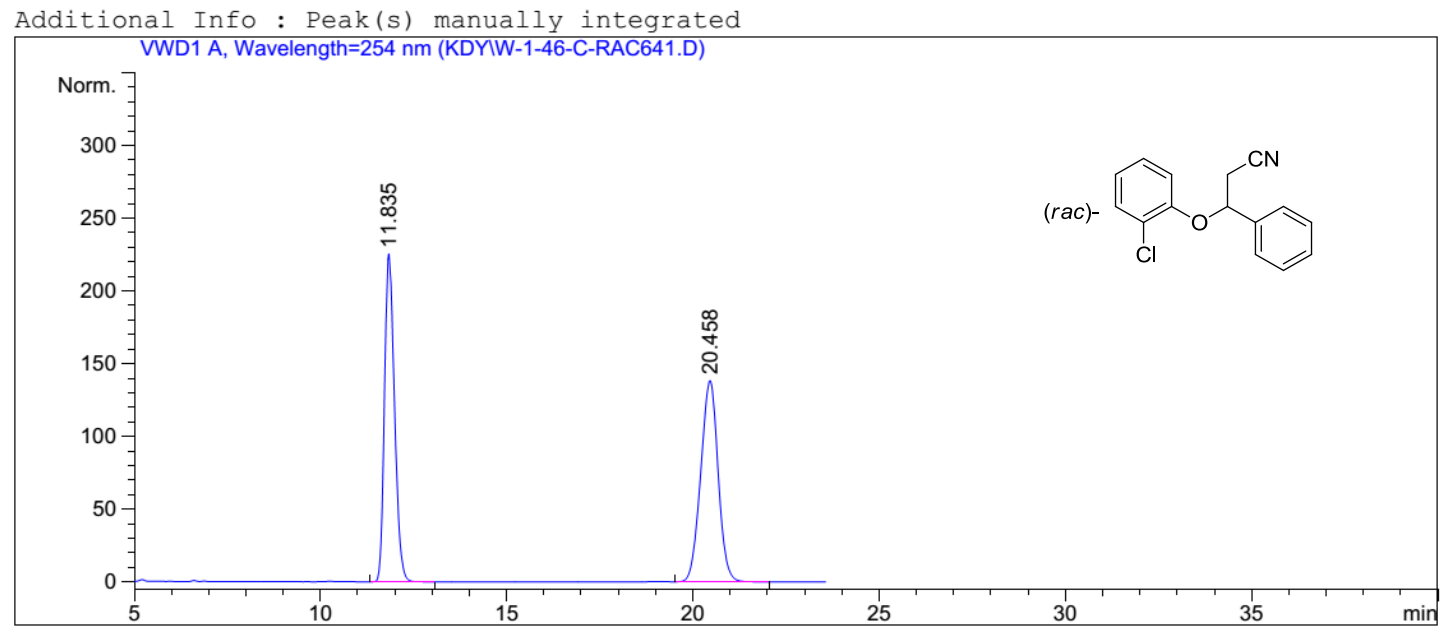

Additional Info : Peak(s) manually integrated
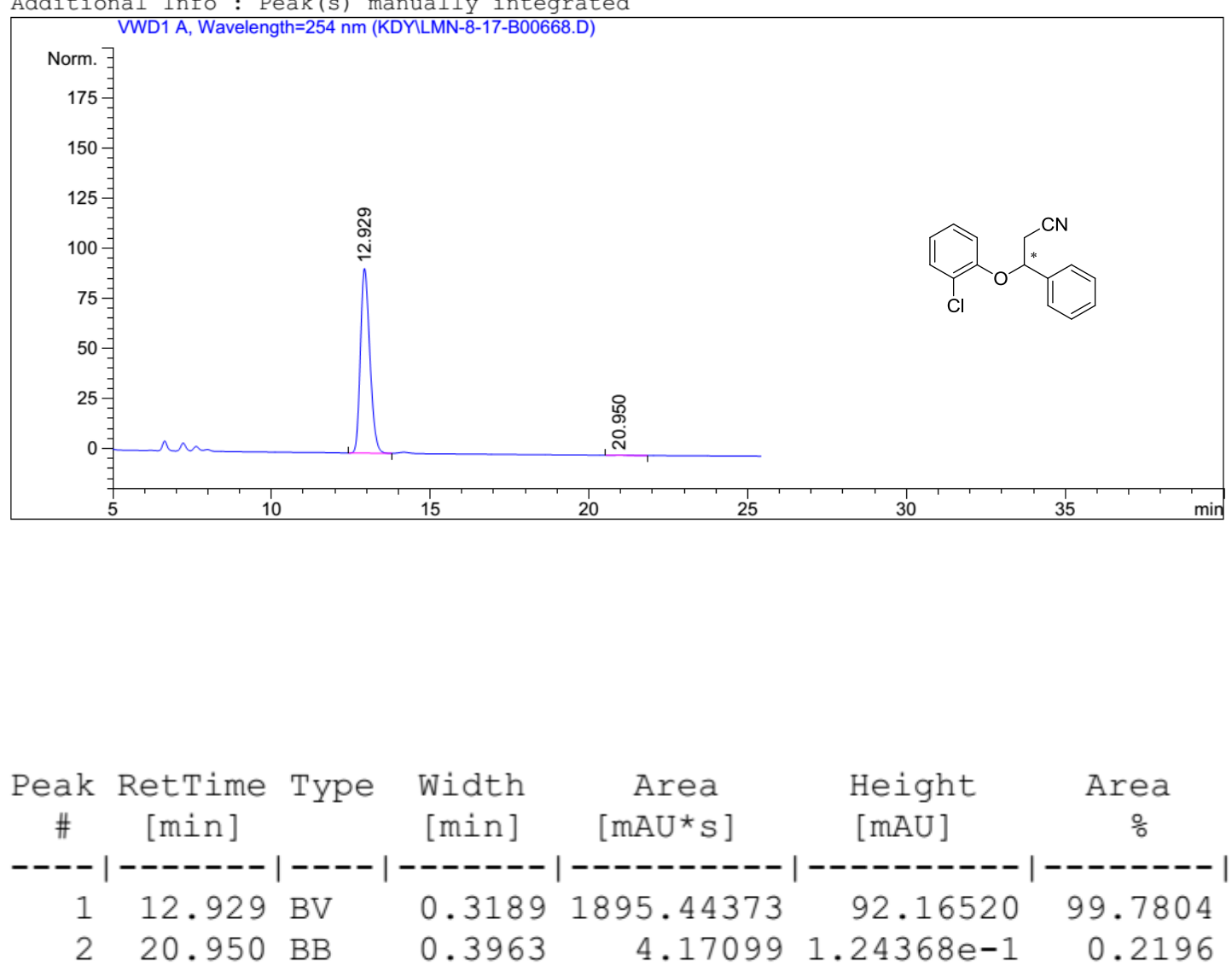

Totals :

$1899.61471 \quad 92.28957$ 
(+)-3-phenyl-3-(p-tolyloxy)propanenitrile (2m)
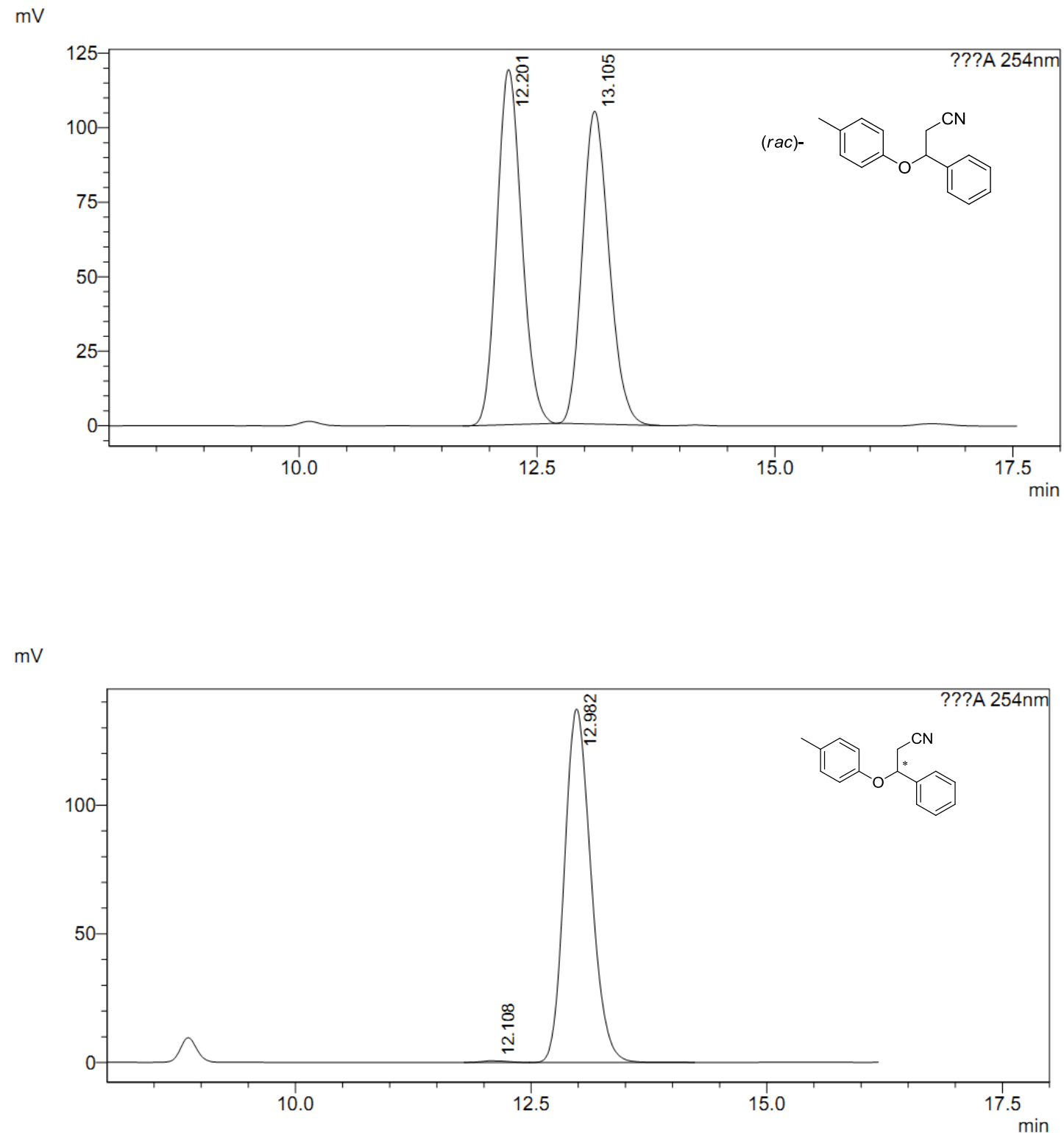

\begin{tabular}{|c|c|c|c|c|}
\hline Peak\# & Ret. Time & Area & Height & Area $\%$ \\
\hline 1 & 12.108 & 12077 & 695 & 0.448 \\
\hline 2 & 12.982 & 2683549 & 137364 & 99.552 \\
\hline Total & & 2695626 & 138059 & 100.000 \\
\hline
\end{tabular}


3-phenyl-3-(p-tolyloxy)propanenitrile $(2 \mathrm{f}+2 \mathrm{~m})$

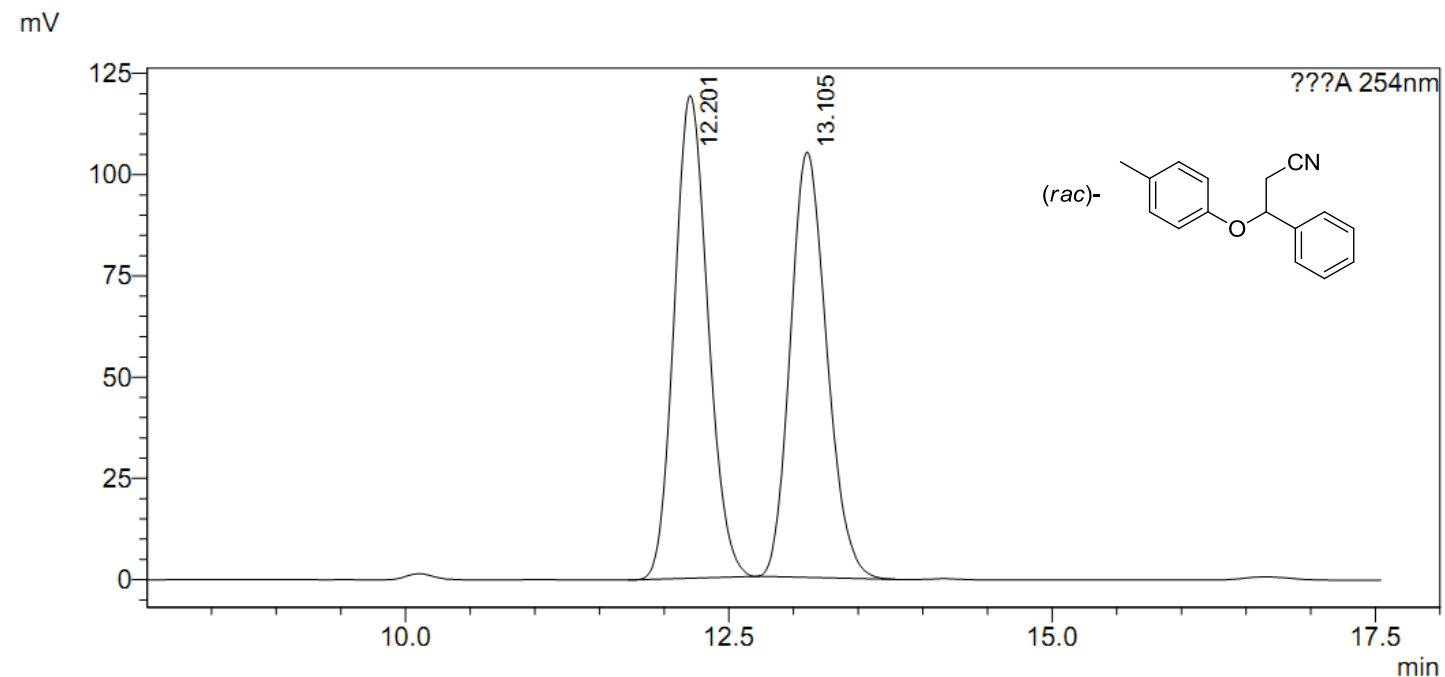

$\mathrm{mV}$

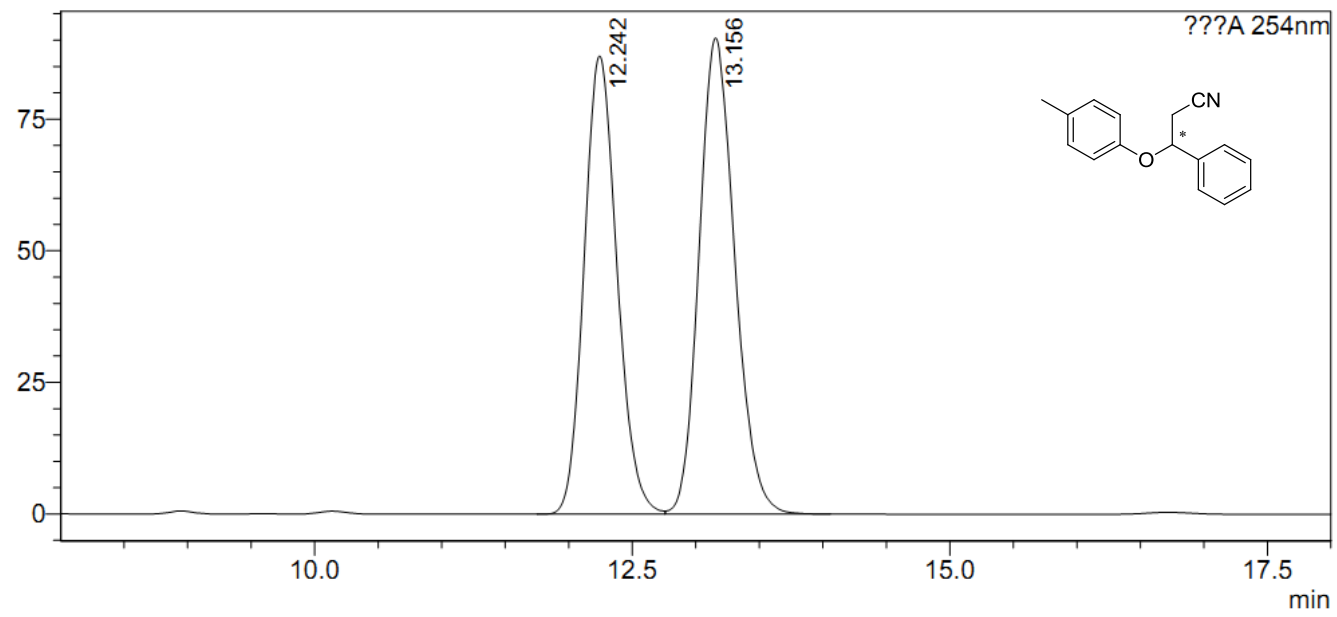

\begin{tabular}{|c|c|c|c|c|}
\hline Peak\# & Ret. Time & Area & Height & Area\% \\
\hline 1 & 12.242 & 1568977 & 87040 & 47.029 \\
\hline 2 & 13.156 & 1767240 & 90443 & 52.971 \\
\hline Total & & 3336217 & 177482 & 100.000 \\
\hline
\end{tabular}




\section{3-methoxy-3-phenylpropanenitrile (2n)}

Additional Info: Peak(s) manually integrated

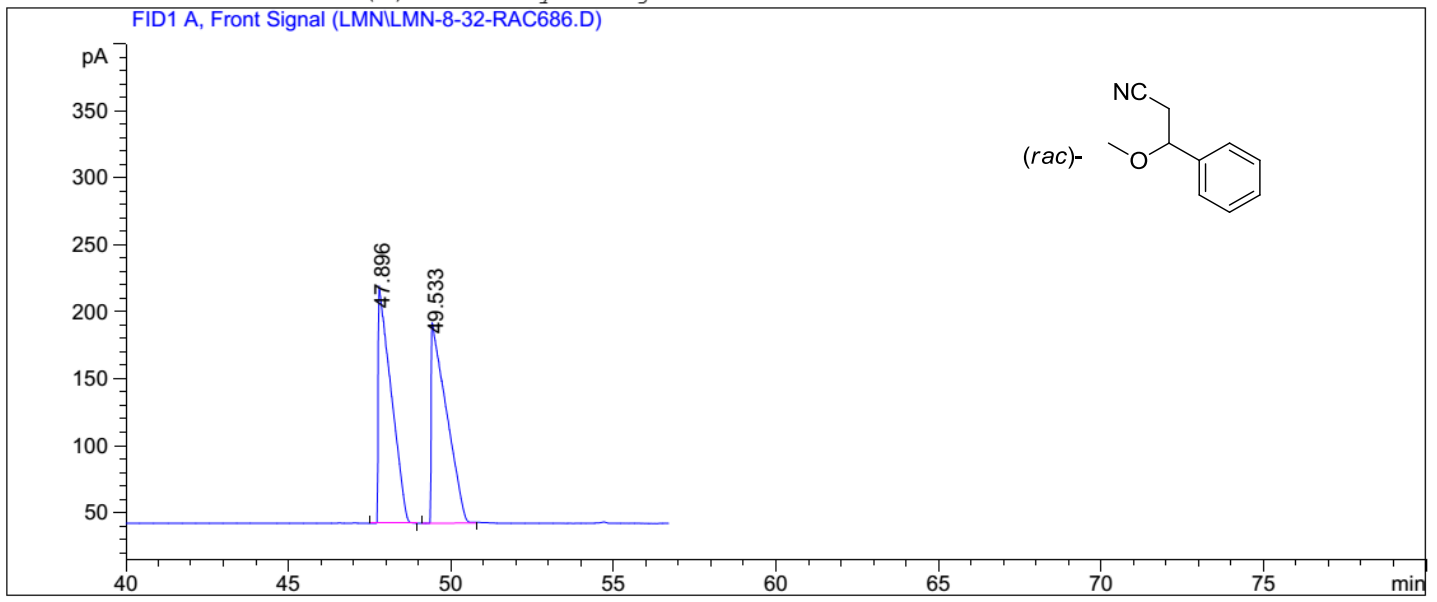

Additional Info : Peak(s) manually integrated

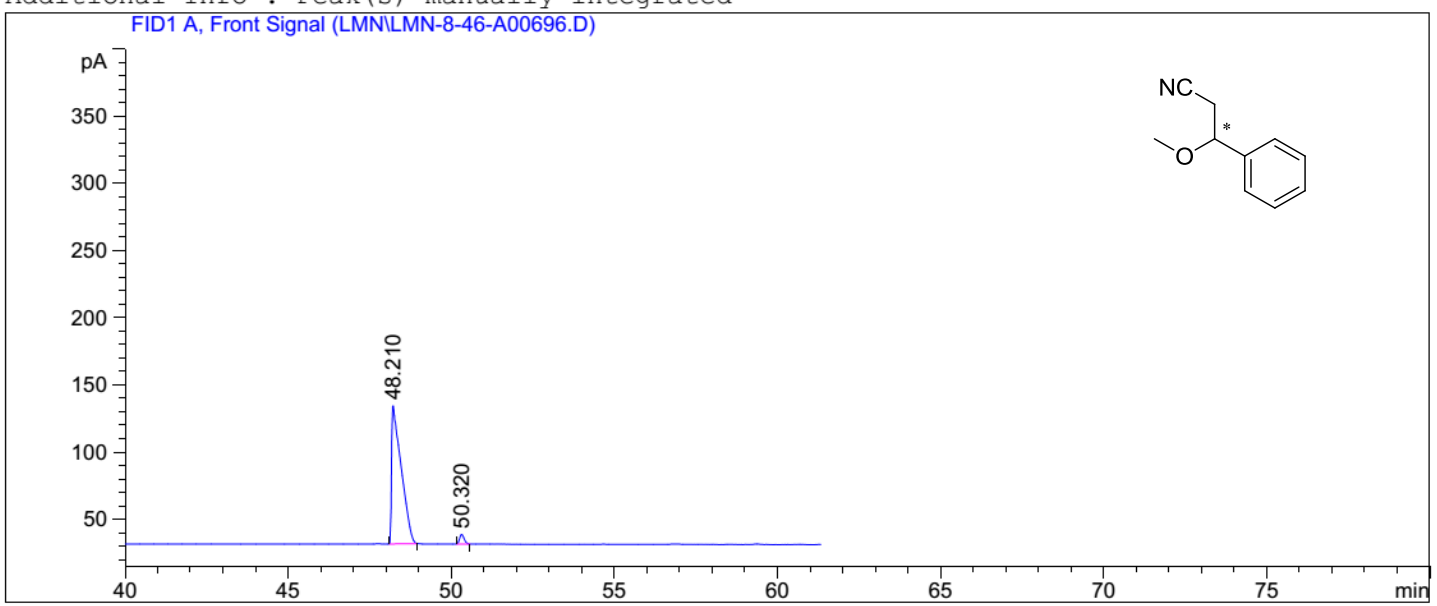

$$
\begin{aligned}
& \text { Peak RetTime Type Width Area Height Area }
\end{aligned}
$$

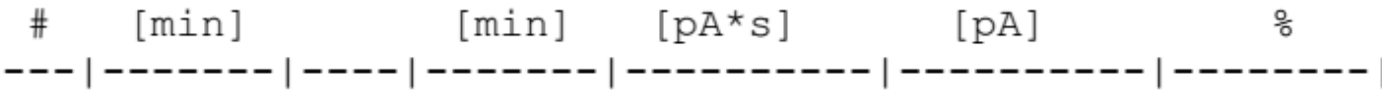

$$
\begin{aligned}
& \begin{array}{llllllll}
1 & 48.210 & \text { BB } & 0.2541 & 2107.38403 & 102.57142 & 96.76736
\end{array}
\end{aligned}
$$

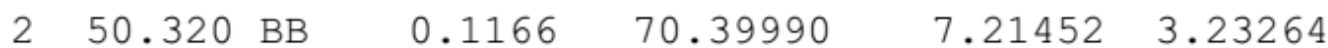

$$
\begin{aligned}
& \text { Totals : } \quad 2177.78394 \quad 109.78594
\end{aligned}
$$




\section{3-isopropoxy-3-phenylpropanenitrile (2o)}
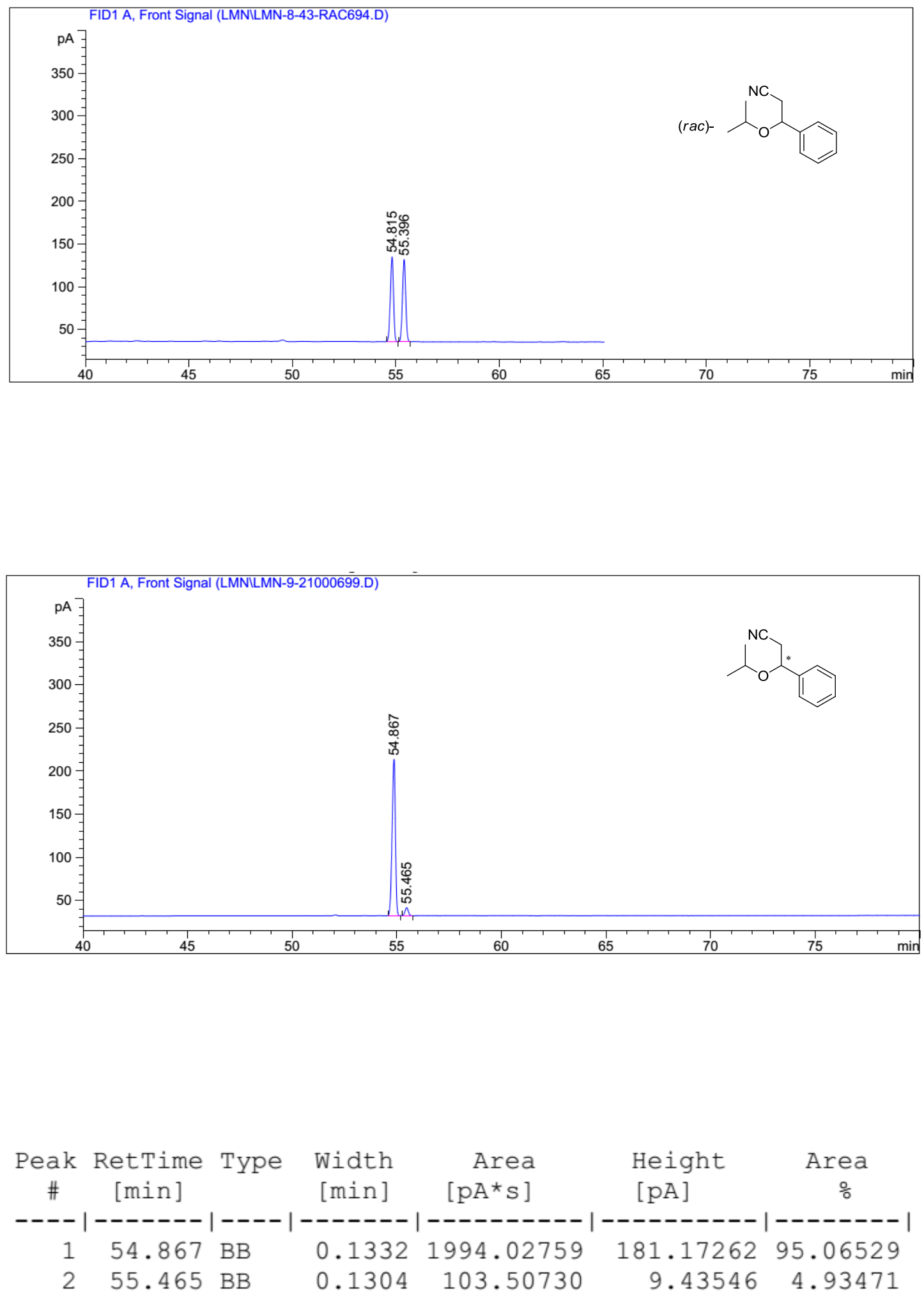

Totals :

$2097.53489 \quad 190.60808$ 


\section{3-phenyl-3-(2,2,2-trifluoroethoxy)propanenitrile (2p)}

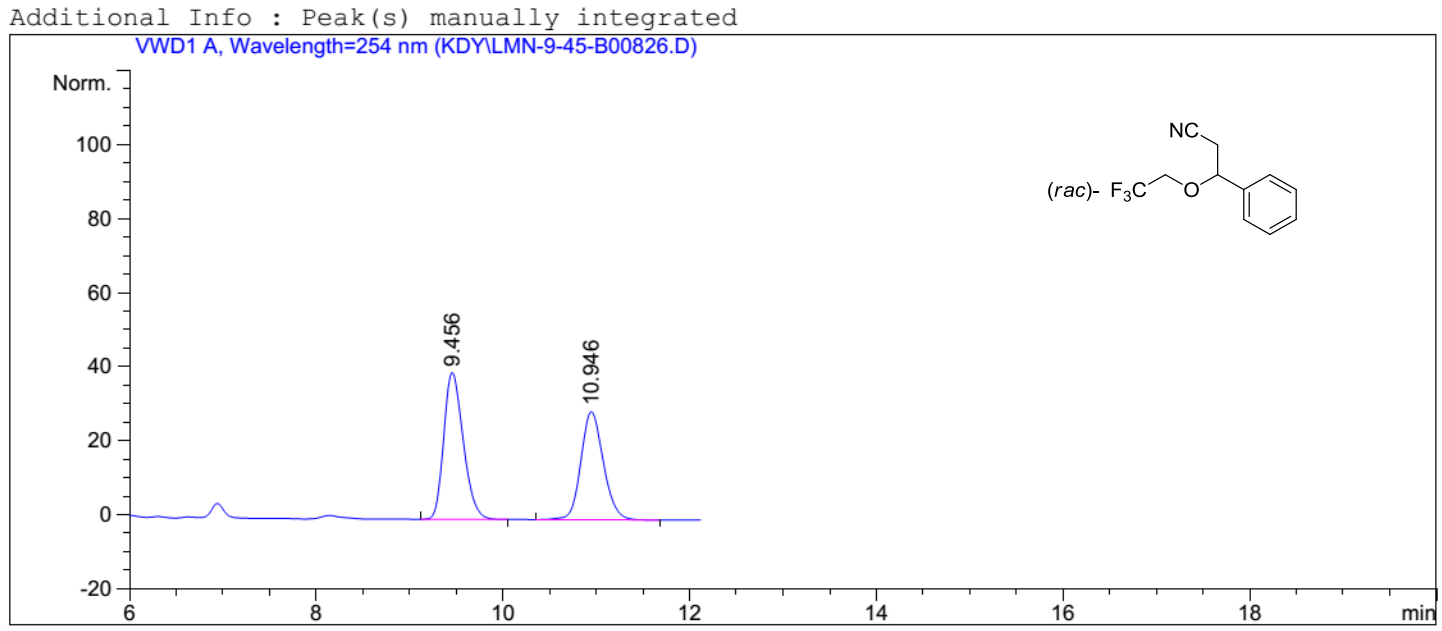

Additional Info : Peak(s) manually integrated

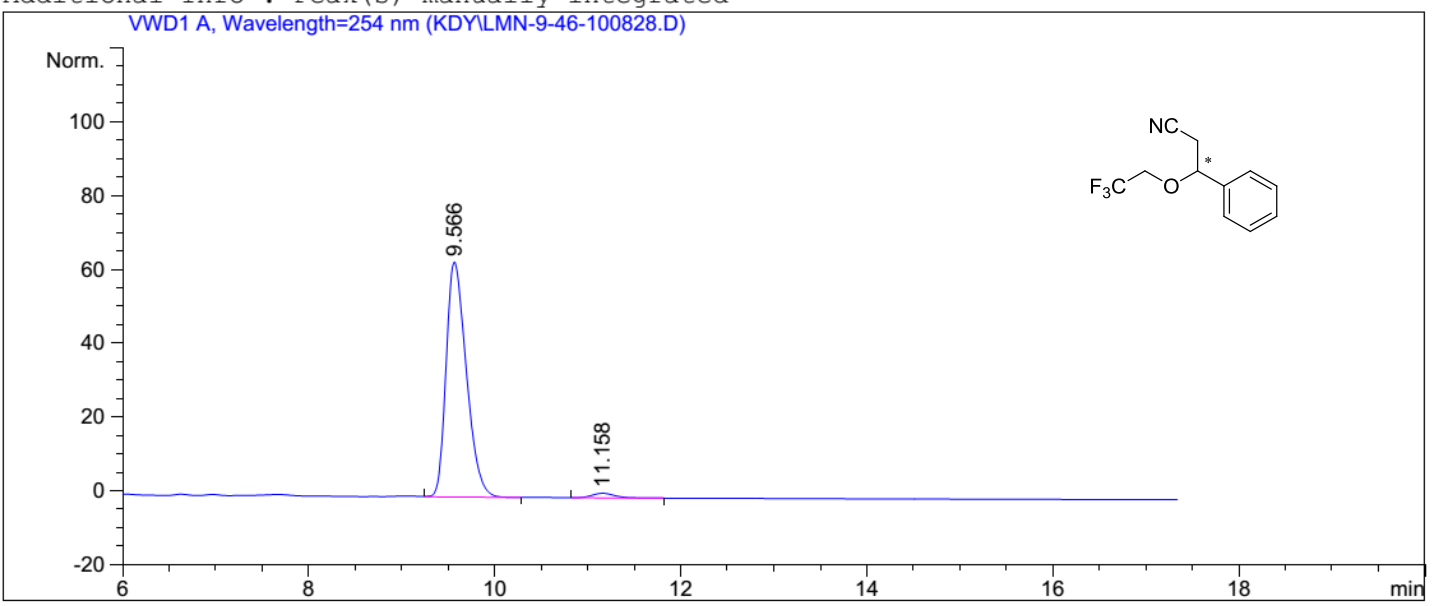

$$
\begin{aligned}
& \text { Peak RetTime Type Width Area Height Area }
\end{aligned}
$$

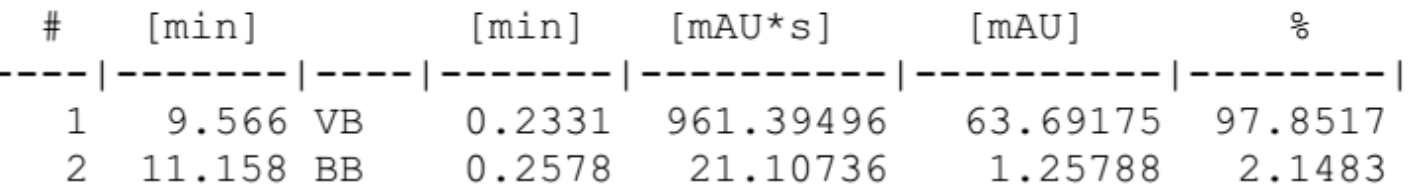

Totals :

$982.50232 \quad 64.94963$ 


\section{3-phenyl-3-(1H-pyrrol-1-yl)propanenitrile (2q)}

Additional Info : Peak(s) manually integrated

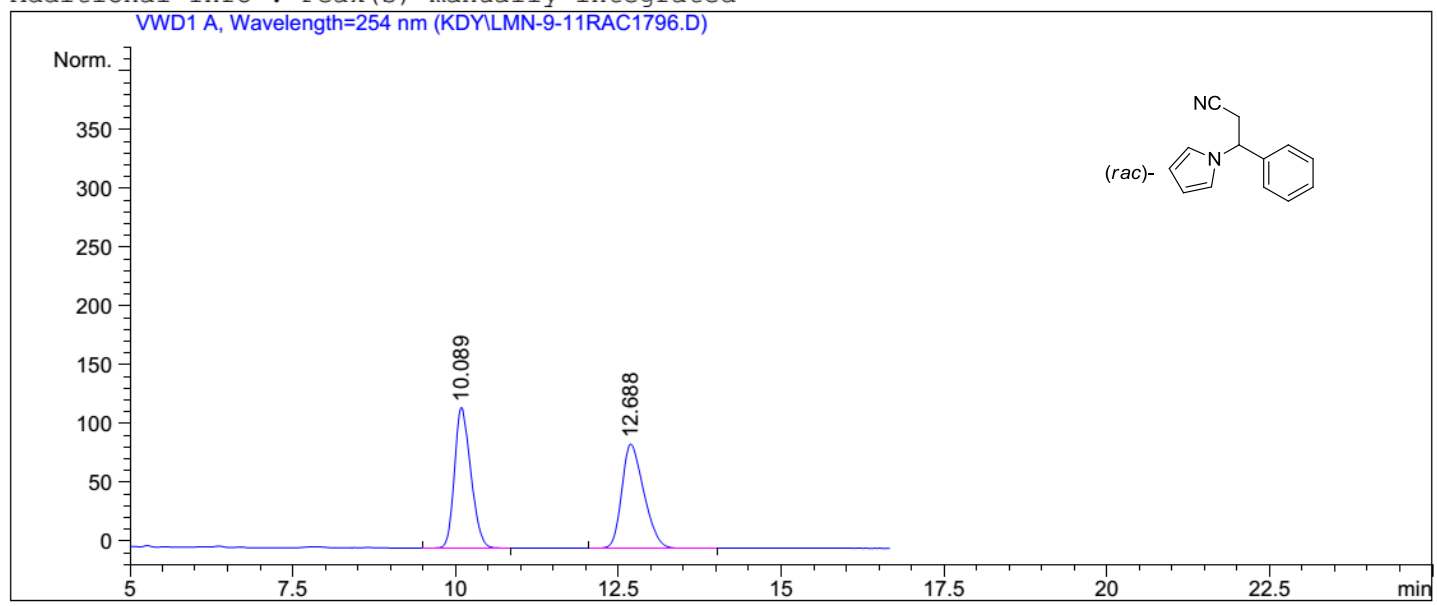

Additional Info: Peak(s) manually integrated

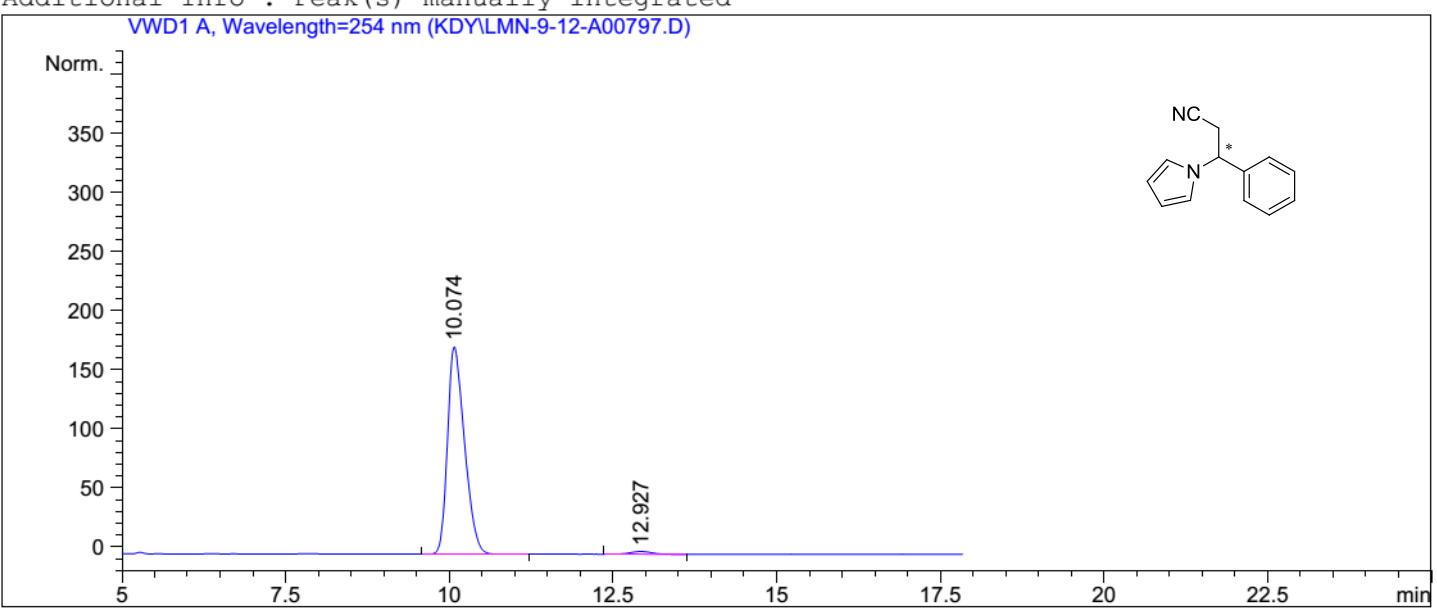

\begin{tabular}{|c|c|c|c|c|c|c|}
\hline $\begin{array}{c}\text { Peak } \\
\#\end{array}$ & $\begin{array}{c}\text { RetTime } \\
\text { [min] }\end{array}$ & Type & $\begin{array}{c}\text { Width } \\
\text { [min] }\end{array}$ & $\begin{array}{c}\text { Area } \\
{\left[\mathrm{mAU}^{*} \mathrm{~S}\right]}\end{array}$ & $\begin{array}{l}\text { Height } \\
\text { [mAU] }\end{array}$ & $\begin{array}{c}\text { Area } \\
\frac{\circ}{0}\end{array}$ \\
\hline---- & & & & ---ー-ー---- & $---------\mid$ & ---1 \\
\hline 1 & 10.074 & $\mathrm{BB}$ & 0.2743 & 3163.14429 & 175.45671 & 5216 \\
\hline 2 & 12.927 & BB & 0.3383 & 47.46604 & 2.16938 & 1.4784 \\
\hline
\end{tabular}

Totals :

$3210.61032 \quad 177.62609$ 


\section{dimethyl (2-cyano-1-(p-tolyl)ethyl)phosphonate (2r)}

Additional Info: Peak(s) manually integrated

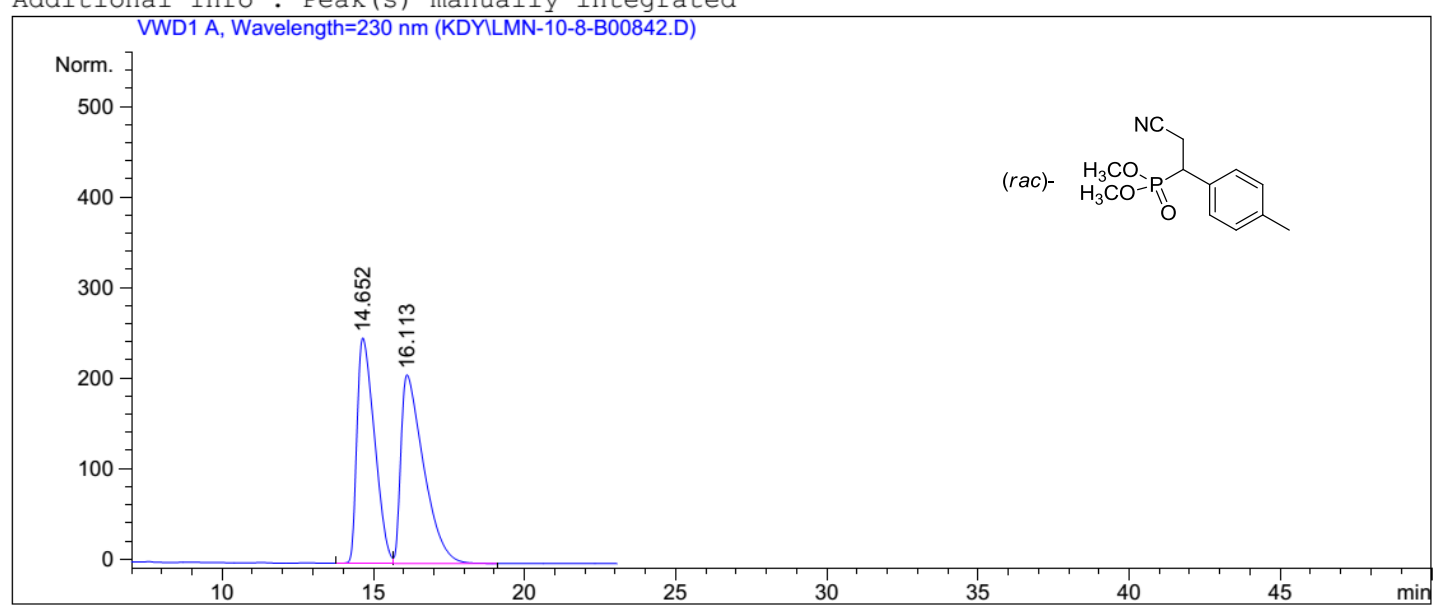

Additional Info : Peak(s) manually integrated
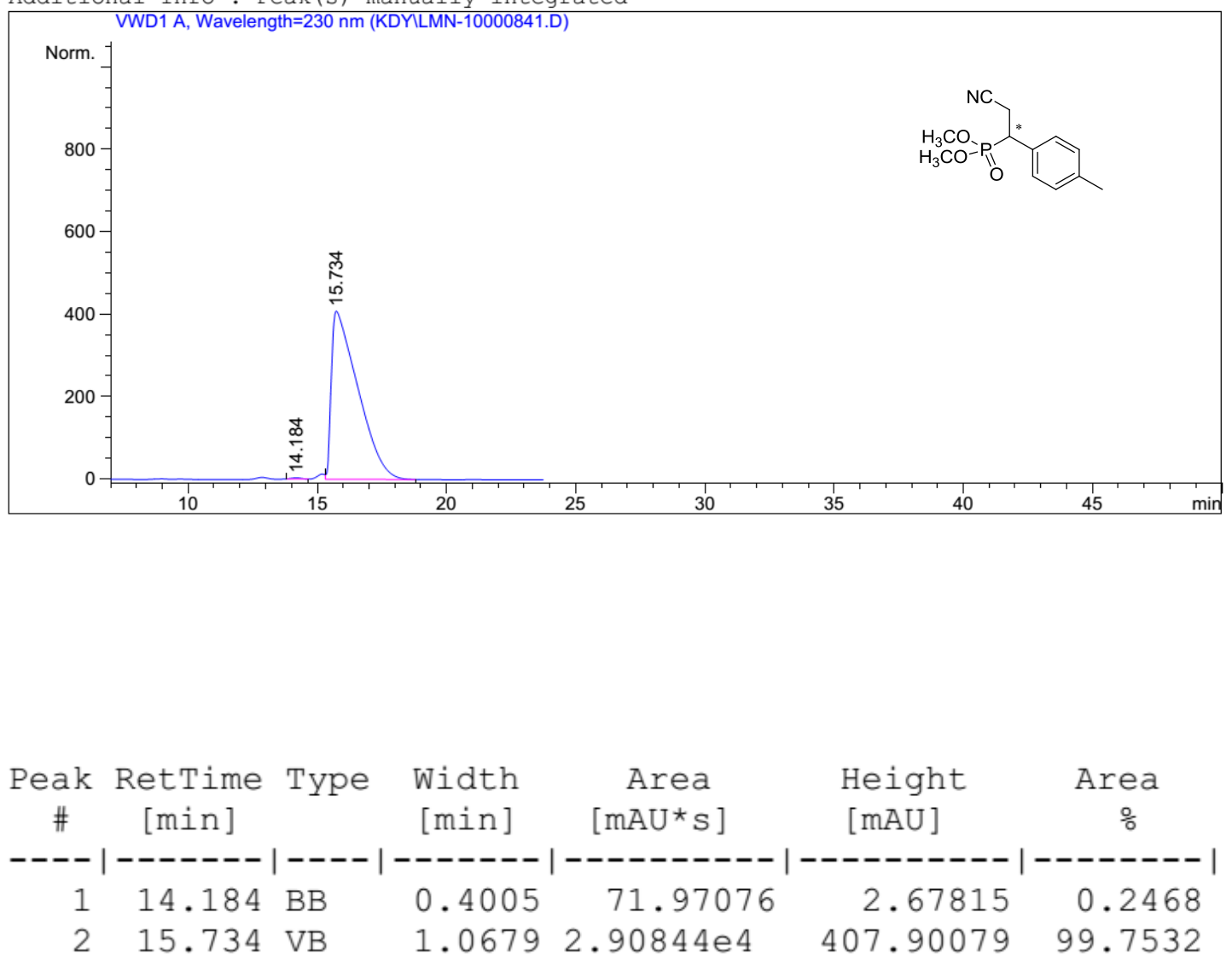

Totals :

$2.91563 e 4 \quad 410.57894$ 
methyl 3-phenoxy-3-phenylpropanoate (4a)

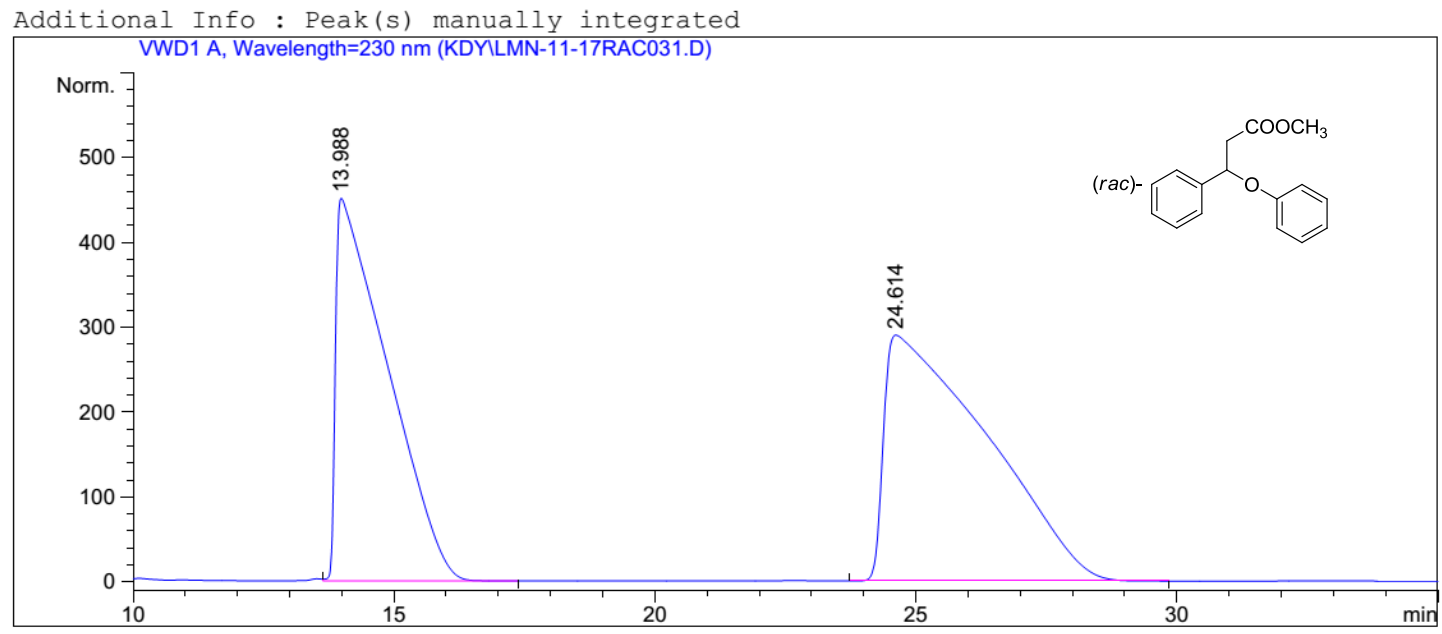

Additional Info : Peak(s) manually integrated
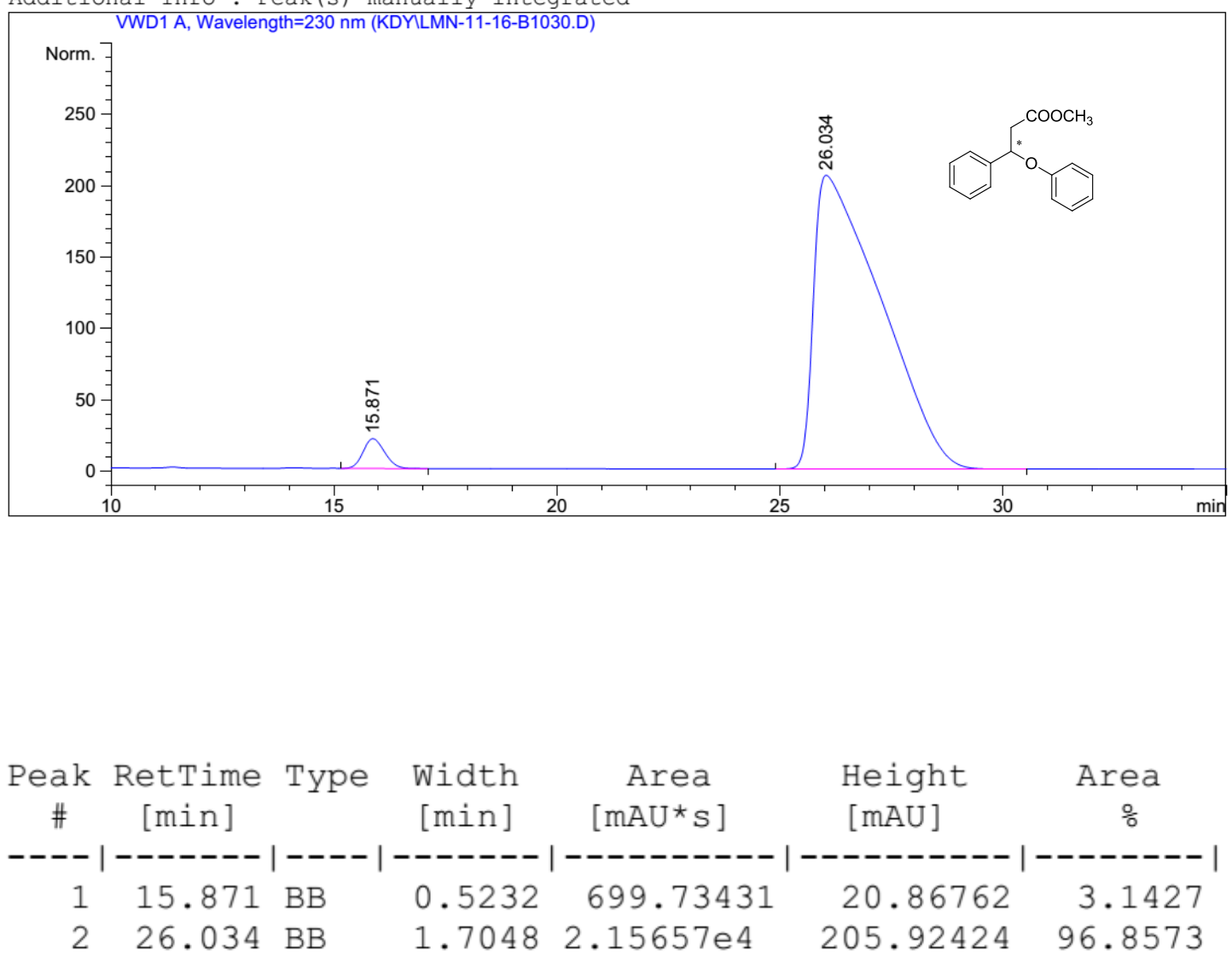

Totals :

$2.22655 e 4 \quad 226.79186$ 
methyl 3-(2-methoxyphenoxy)-3-phenylpropanoate (4b)

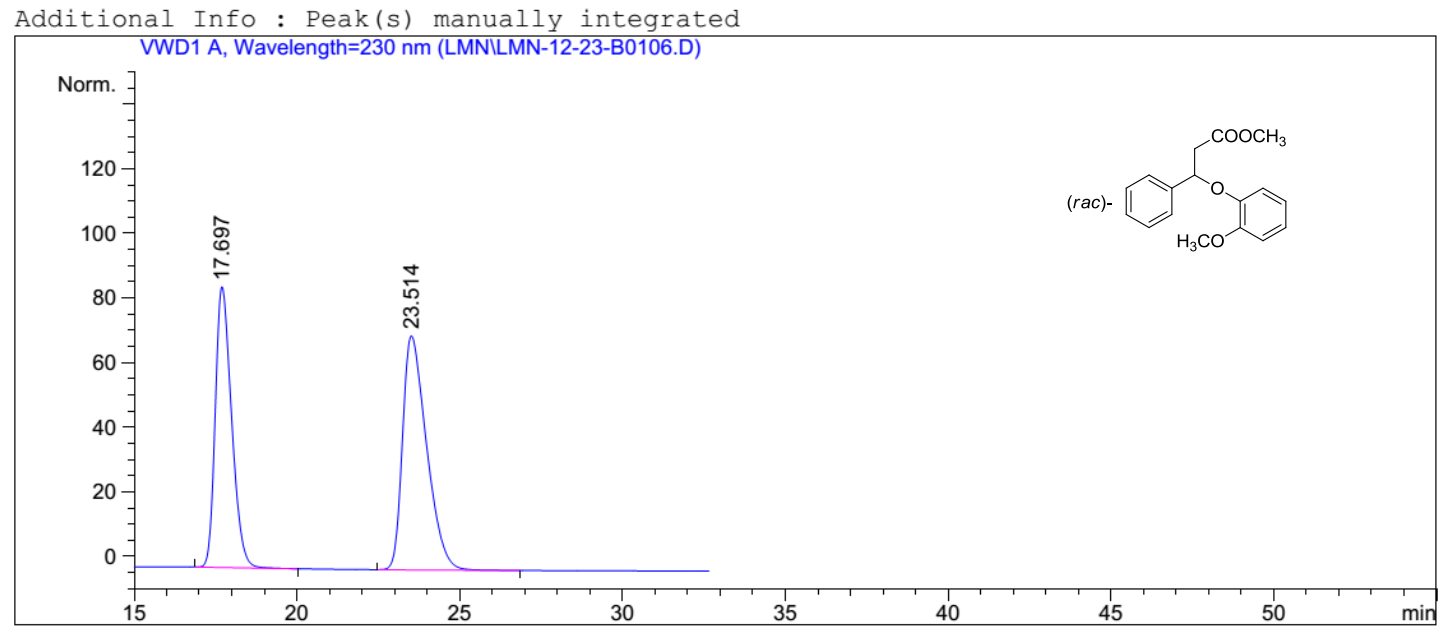

Additional Info : Peak(s) manually integrated
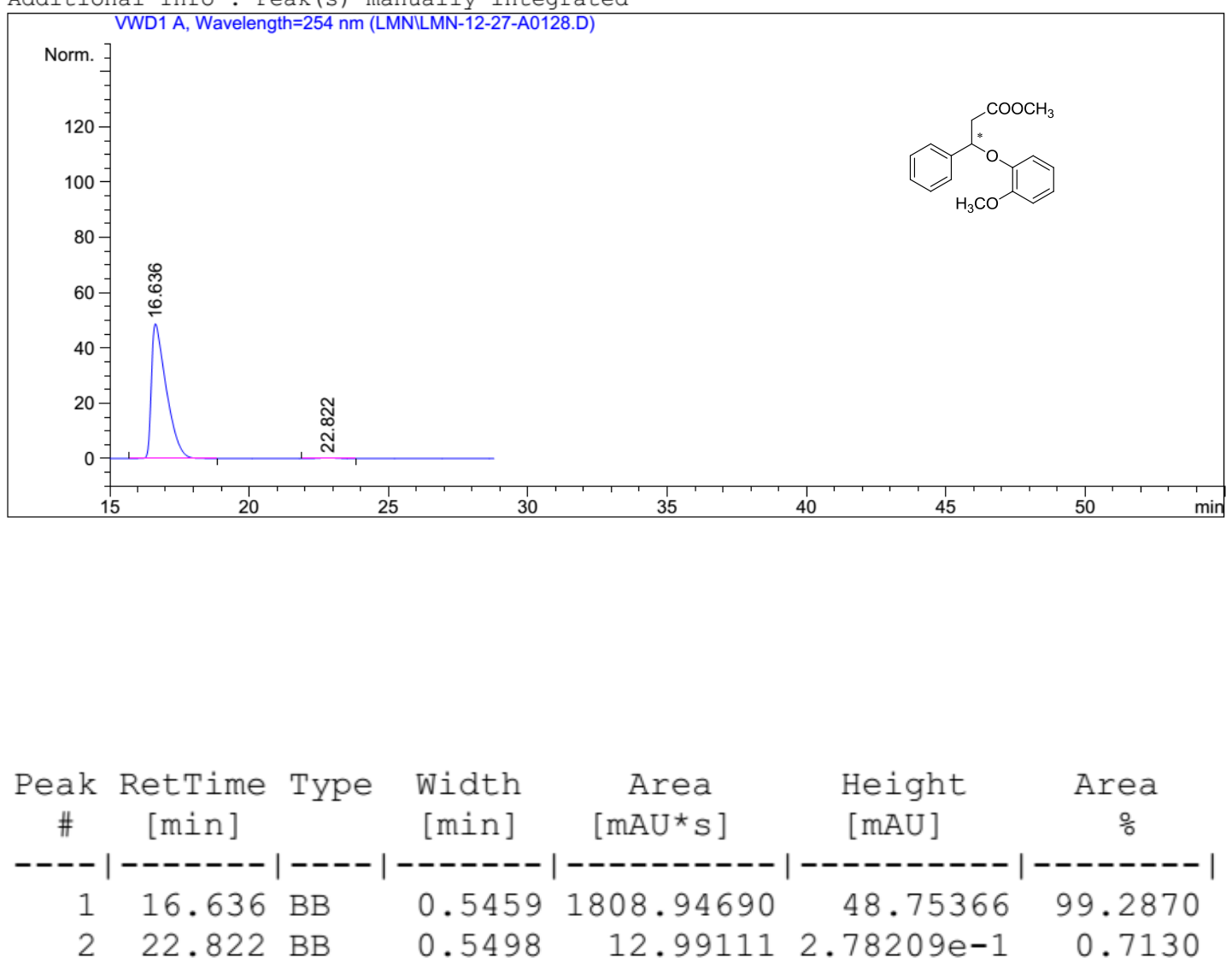

Totals: $\quad 1821.93801 \quad 49.03187$ 
methyl 3-phenyl-3-(o-tolyloxy)propanoate (4c)

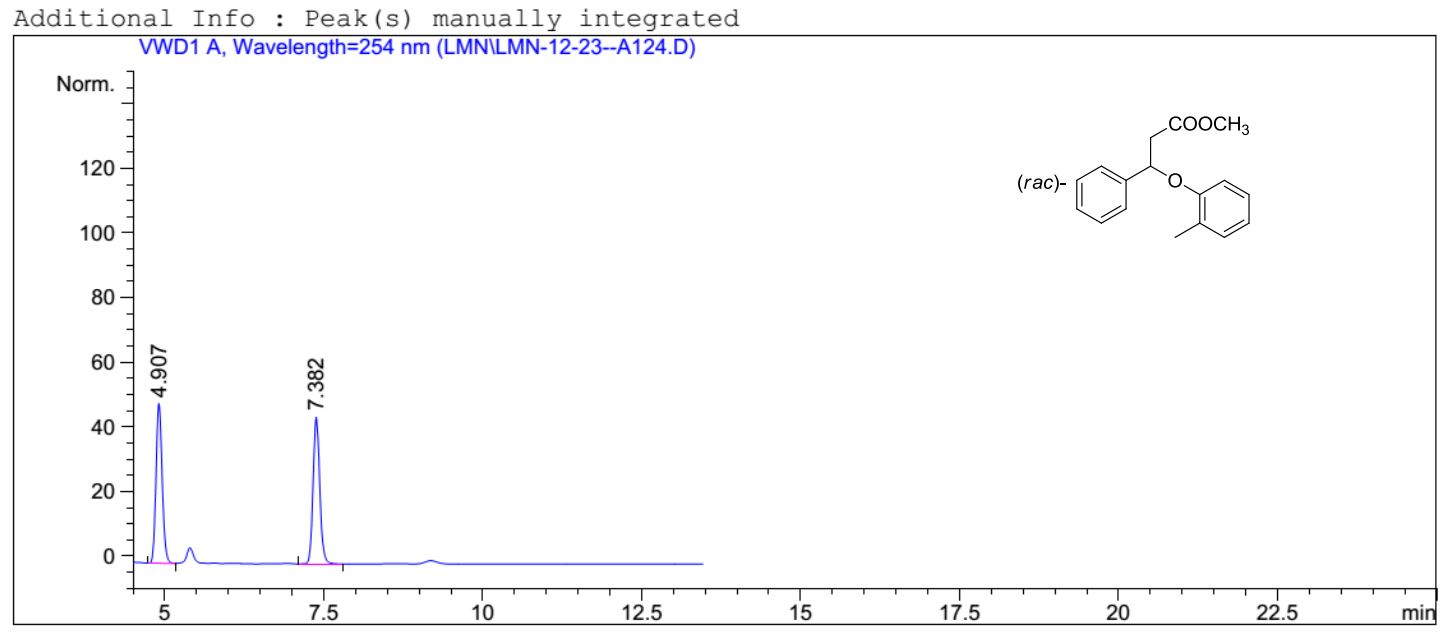

Additional Info : Peak(s) manually integrated
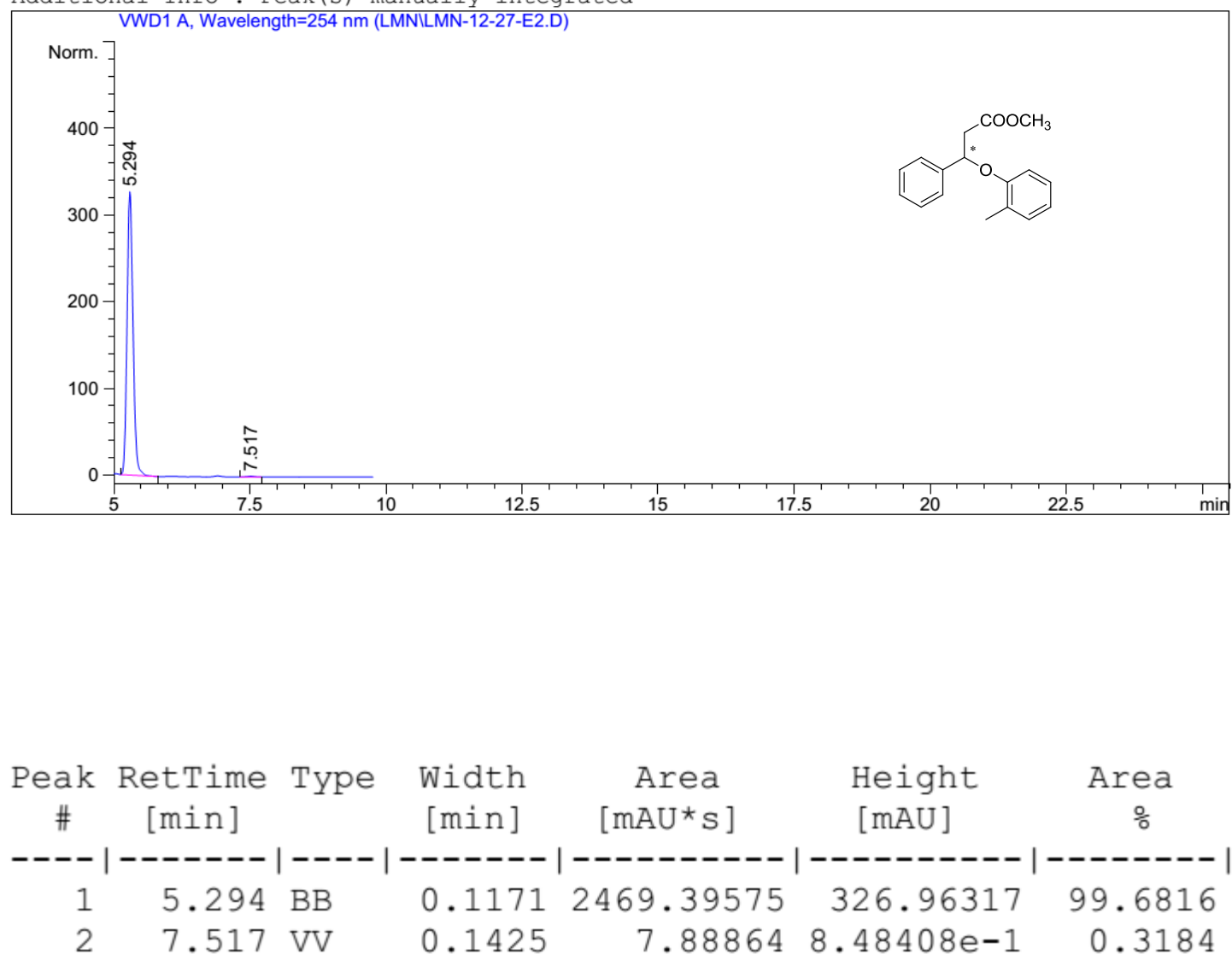

Totals :

$2477.28440 \quad 327.81157$ 
(+)-methyl 3-phenyl-3-(p-tolyloxy)propanoate (4d)
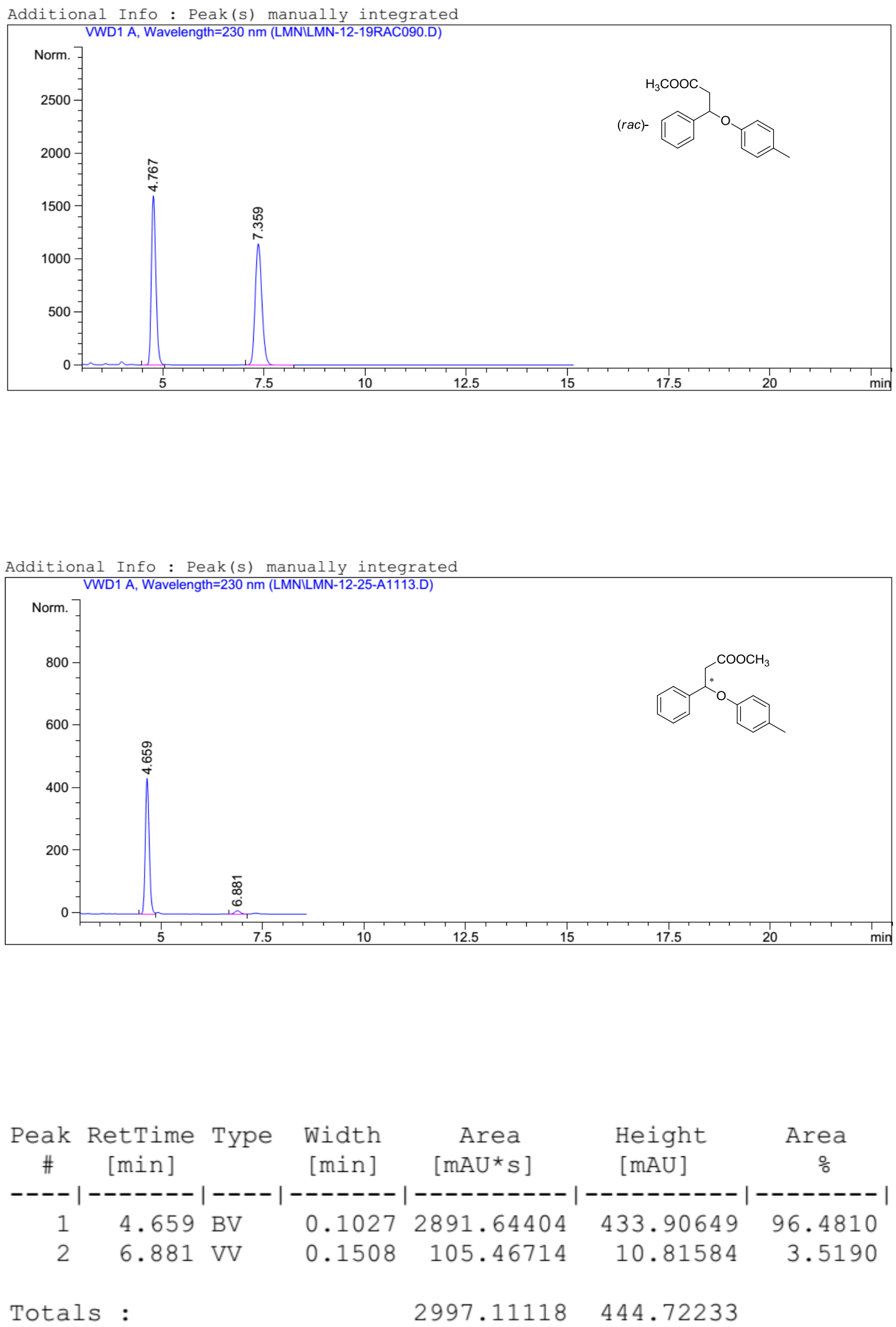


\section{(-)-methyl 3-phenyl-3-(p-tolyloxy)propanoate ( 4e)}
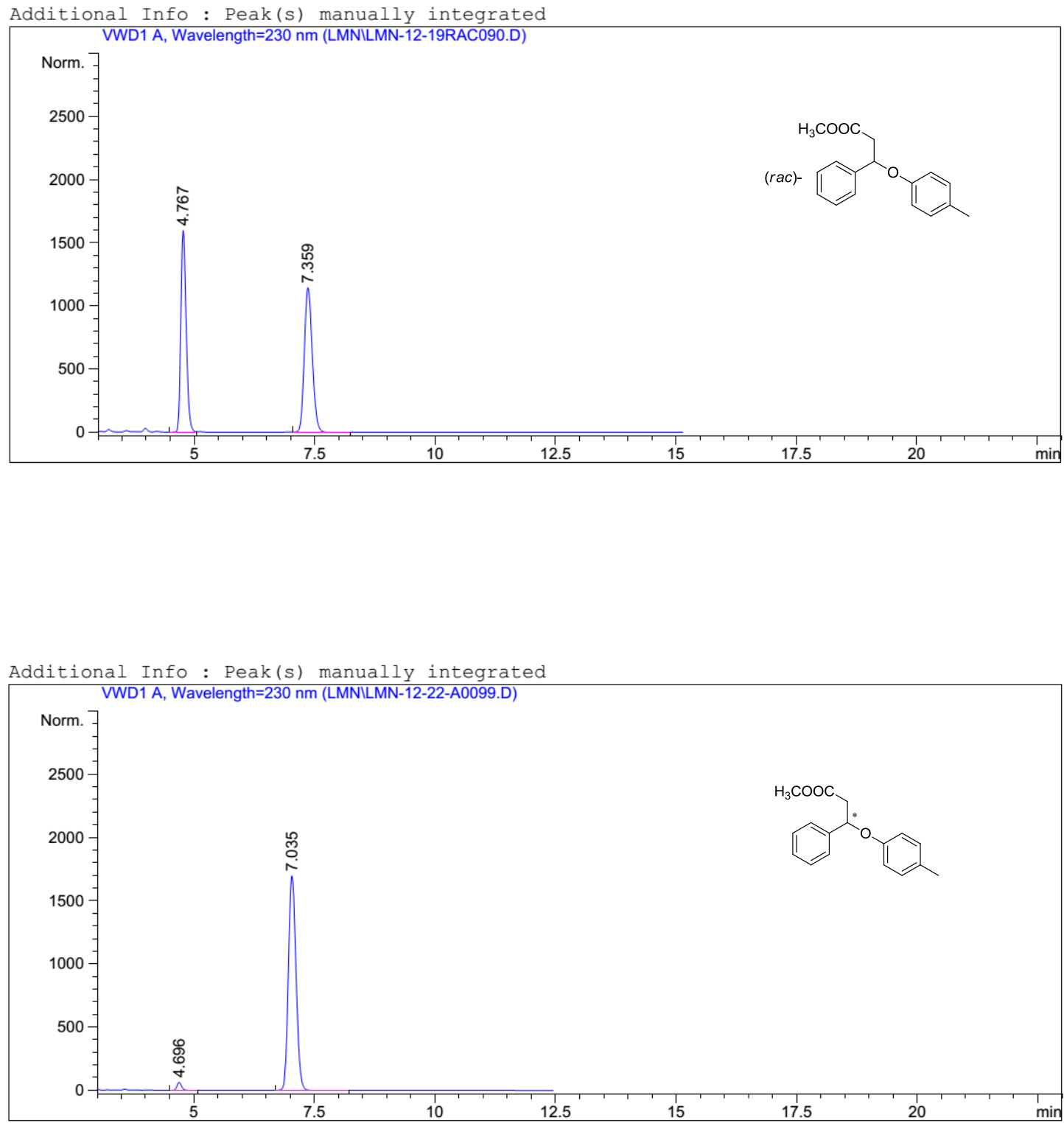

\begin{tabular}{|c|c|c|c|c|c|c|}
\hline $\begin{array}{c}\text { Peak } \\
\#\end{array}$ & $\begin{array}{c}\text { RetTime } \\
\text { [min] }\end{array}$ & Type & $\begin{array}{l}\text { Width } \\
\text { [min] }\end{array}$ & $\begin{array}{c}\text { Area } \\
{\left[\mathrm{mAU}^{\star} \mathrm{s}\right]}\end{array}$ & $\begin{array}{l}\text { Height } \\
\text { [mAU] }\end{array}$ & $\begin{array}{c}\text { Area } \\
\%\end{array}$ \\
\hline--- & ------- & $1=-$ & ------- & |---------- & | ---------- & -------- \\
\hline 1 & 4.696 & BV & 0.1032 & 420.70148 & 62.78996 & 2.1952 \\
\hline 2 & 7.035 & VB & 738 & $1.87441 \mathrm{e} 4$ & 1698.23267 & 97.8048 \\
\hline a. & & & & $1.91648 \mathrm{e} 4$ & 1761.0 & \\
\hline
\end{tabular}


methyl 3-(4-chlorophenoxy)-3-phenylpropanoate (4f)
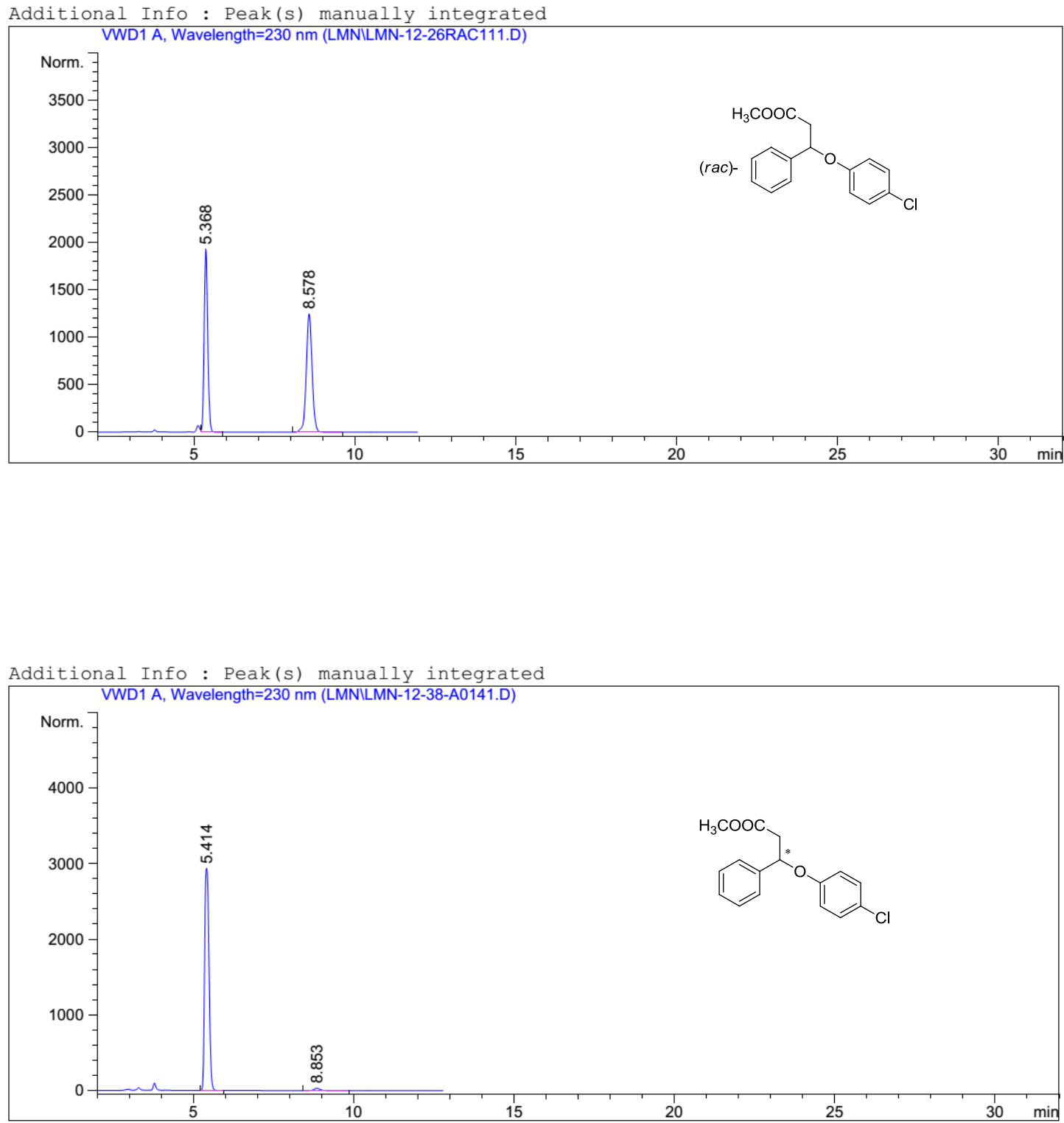

$$
\begin{aligned}
& \text { Peak RetTime Type Width Area Height Area }
\end{aligned}
$$

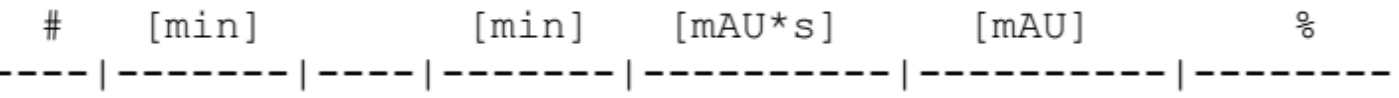

$$
\begin{aligned}
& \begin{array}{lllllll}
1 & 5.414 & \text { BB } & 0.1509 & 2.76903 e 4 & 2940.85962 & 98.5740
\end{array} \\
& \begin{array}{lllllll}
2 & 8.853 & \text { BB } & 0.1941 & 400.56424 & 31.76948 & 1.4260
\end{array}
\end{aligned}
$$




\section{ethyl 3-phenoxybutanoate (4g)}
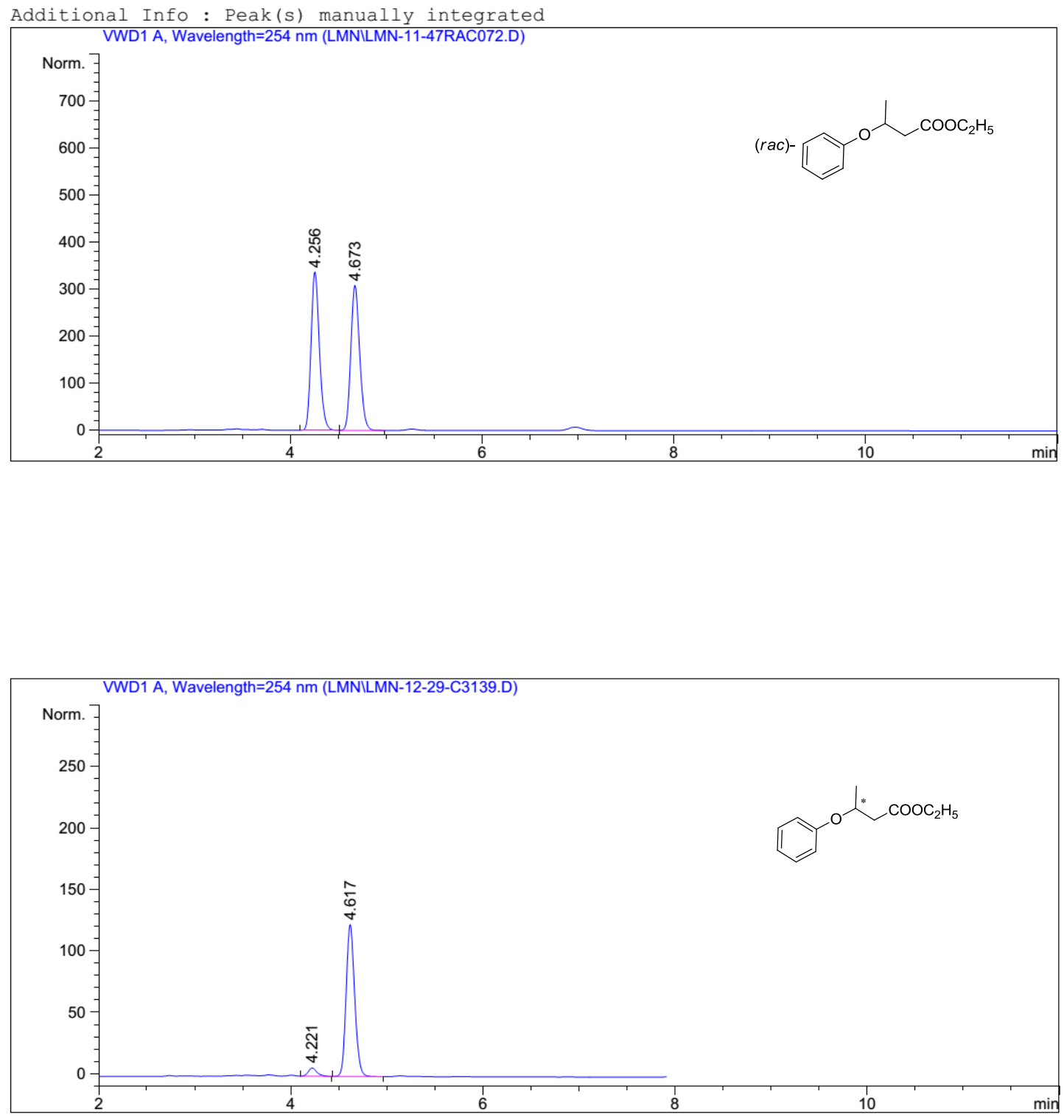

\begin{tabular}{|c|c|c|c|c|c|c|}
\hline $\begin{array}{c}\text { Peak } \\
\quad \#\end{array}$ & $\begin{array}{c}\text { RetTime } \\
\text { [min] }\end{array}$ & Type & $\begin{array}{c}\text { Width } \\
\text { [min] }\end{array}$ & $\begin{array}{c}\text { Area } \\
{\left[\mathrm{mAU}^{\star} \mathrm{s}\right]}\end{array}$ & $\begin{array}{l}\text { Height } \\
\text { [mAU] }\end{array}$ & $\begin{array}{c}\text { Area } \\
\frac{\circ}{0}\end{array}$ \\
\hline-- & $-------\mid$ & --1 & ----- & --------- & ----ー-ー- & -------- \\
\hline 1 & 4.221 & $\mathrm{BB}$ & 0.0934 & 42.22136 & 6.80440 & 5.2444 \\
\hline 2 & 4.617 & $\mathrm{BB}$ & 0.0950 & 762.84778 & 123.66019 & 94.7556 \\
\hline tal & & & & 805.06913 & 6459 & \\
\hline
\end{tabular}


3-(2-methoxyphenoxy)- $N$-methyl-3-phenylpropan-1-amine ((S)-Nisoxetine)

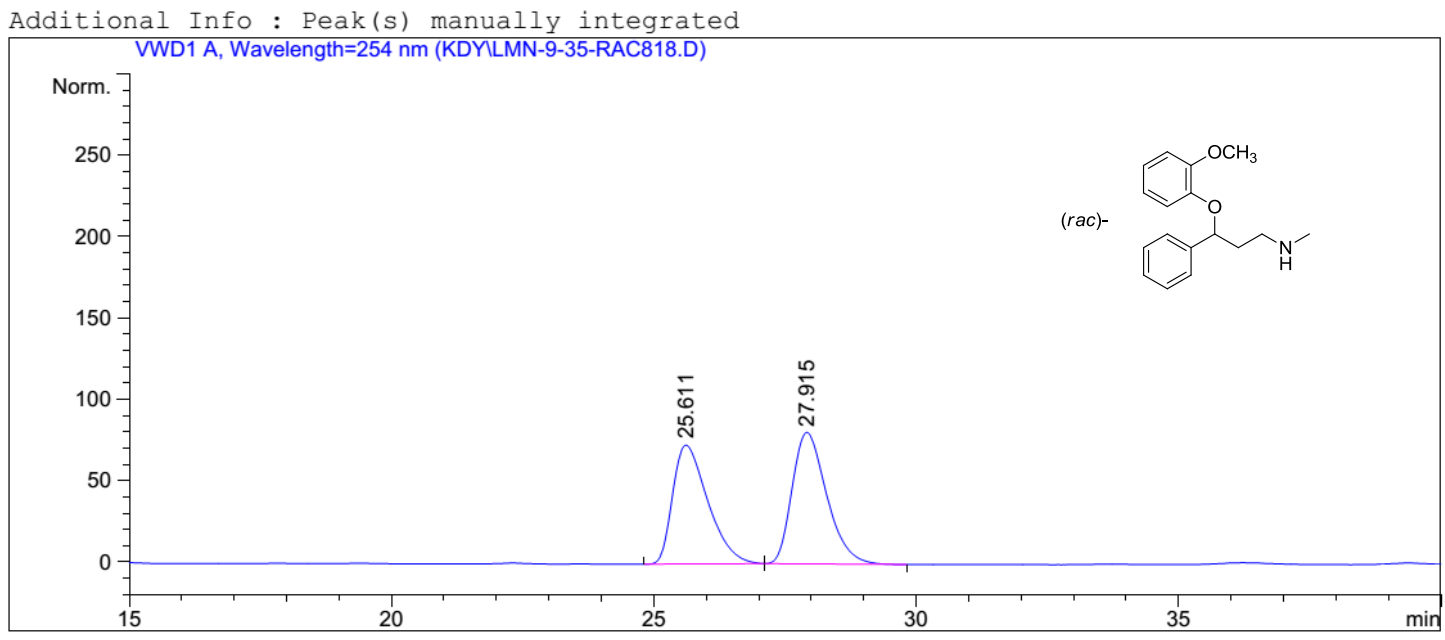

Additional Info : Peak(s) manually integrated
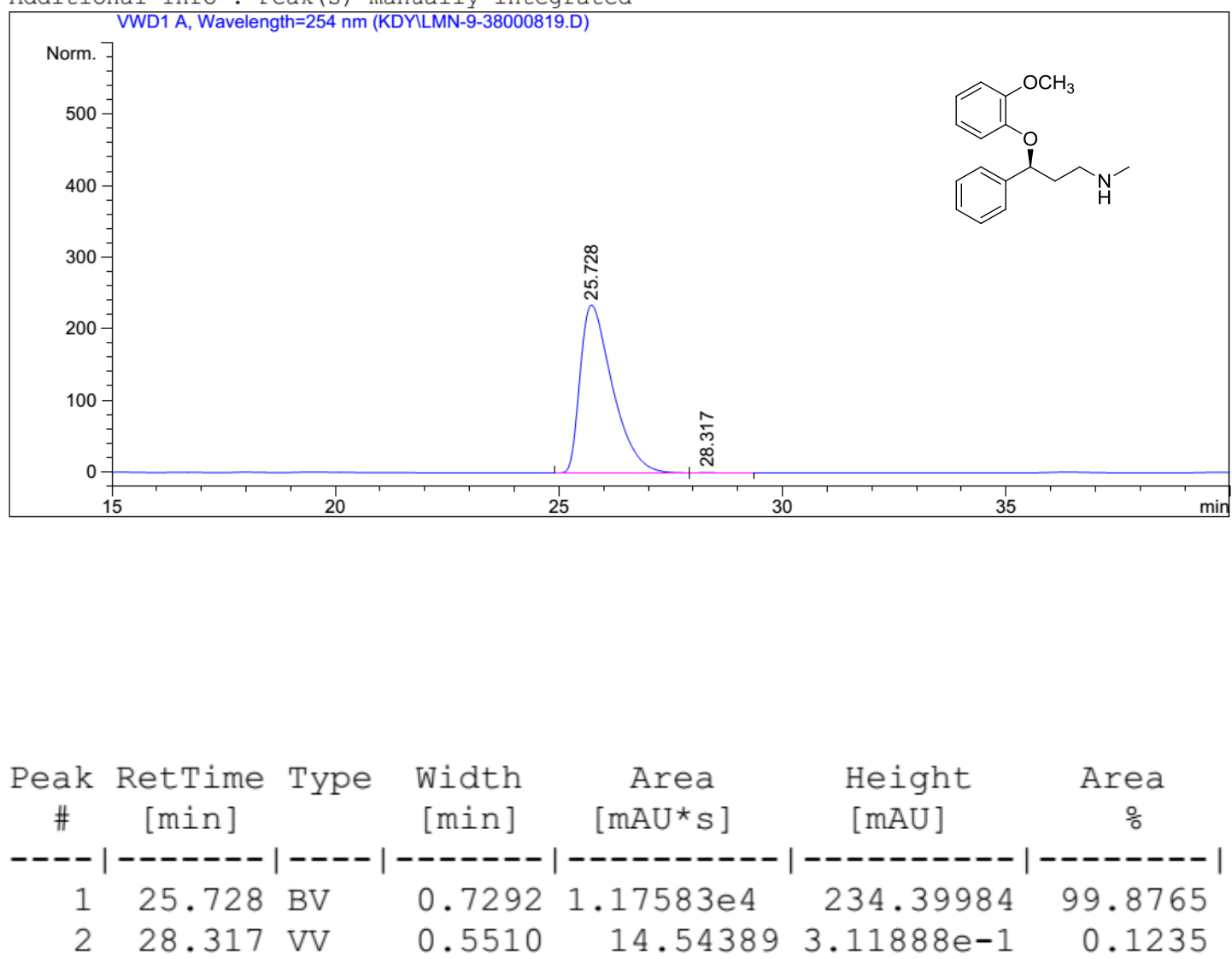

Totals :

$1.17729 \mathrm{e} 4 \quad 234.71173$ 